$$
4
$$

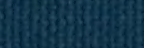




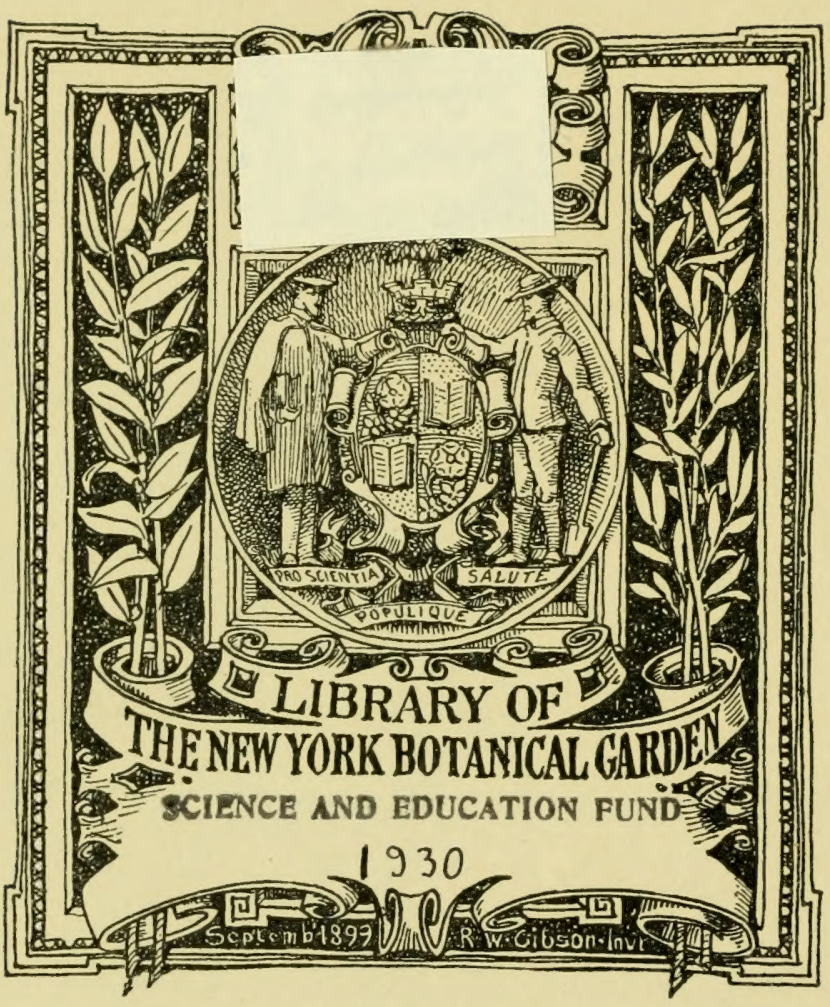








\section{ÉTUDES MÉDICALES}

SUR

\section{LES GENRES}

\section{ARUM ET ACTEA}

PAR

$\mathrm{M}^{\text {LLe }} \mathrm{J}$. CHAULiaguet

DOCTEUR EN MÉDECINE

(Travail dı Laboraloire d'Histoire naturelle de la Faculté de Médecine)

(Paru le 8 Avril 1897)

\section{PARIS}

IMPRIMERIE F. LEVÉ

17, RUE CASSETTE, 17

1897 





\title{
ETUDES MÉDICALES
}

SUR

\section{LES GENRES}

\section{ARUM Eт ACTEA}

\author{
PAR
}

\author{
M ${ }^{\text {LIE }}$ J C CHAULIAGUET \\ DOCTEUR EN MÉDEGINE
}

(Travail du Laboratoire d'Histoire naturelle de la Faculté de Médecine) (Paru le 8 Avril 1897)

\section{PARIS}

IMPRIMERIE F. LEVÉ

17, RUE CASSETTE, 17 


$$
\begin{aligned}
& x^{2} \\
& 8 \times 5
\end{aligned}
$$




\section{AVANT-PROPOS}

Le travail que nous avons l'honneur de présenter à la Faculté est essentiellement un travail de Botanique médicale. Son but est d'élucider l'histoire botanique, chimique, physiologique et médicale de plantes réputées toxiques ou médicamenteuses.

Il peut paraître singulier de voir réunir en seul travail deux études distinctes, relatives à des plantes appartenant à deux groupes aussi éloignés que les Arui et les Actex. Celles-ci présenteni plutôt un intérèt thérapeutique, ceux-là un intérêt toxicologique et médico-légal. La raison de cette réunion est simple. Nos recherches, entreprises il y a deux ans, le furent sans le but arrêté d'y trouver un sujet de thèse. Les quelques conclusions qui s'en dégagent ont paru à II. Heim au laboratoire duquel ces recherches furent poursuivies), capables de montrer l'intérêt que peuvent présenter, pour les diverses branches des sciences médicales, des études botaniques orientées dans cette direction spéciale. C'est sur son conseil que cesrecherches ont été réunies en un seul travail.

Est-il besoin d'ajouter que ce n'est qu'aux conseils de ceux qui ont bien voulu nous guider dans ces recherches que nous devons les résultats obtenus? Ce nous est un bien doux devoir de placer leurs noms en tête de cet essai.

II. le Professeur Brouardel nous fait le grand honneur de vouloir bien accepter la présidence de notre thèse. Nous ne 
saurions trop lui en exprimer tonte notre respectueuse gratitude.

C'est dans le service de MI. Dalché, médecin des hôpitaux, qu'ont été recueillies les observations cliniques relatives à l'Actée. Son bienveillant accueil et ses précieux conseils ne. nous laisseront point oublier le temps passé à tenter, sous sa direction, ces essais dont il a bien voulu contrôler J'exactitude.

Ce n’est qu’à l'habile collaboration de M. Ilébert, préparateur aux travaux pratiques de chimie de la Faculté, que nous devons d'avoir pu élucider diverses questions chimiques, d'une importance primordiale pour nos recherches. C'est en son nom et au nôtre que nous présentons les résultats consignés dans la partie chimique de ce travail.

Toutes nos études ont été poursuivies au laboratoire d'Ilistoire naturelle de la Faculté, sur les conseils et sous la direction constante de M. IIeim, qui a bien voulu suivre toutes nos observations et nous prêter son aide dans toutes les expériences délicates de physiologie. Il n’est presque pas un fait consigné dans ce travail qu'il n’ait tenu à vérifier par lui-même. Les vieilles relations d'intimité qui unissent nos familles rendraient banale l'expression de notre reconnaissance.

Nous ne saurions oublier la bienveillance que nous ont lémoignée, pendant notre séjour dans leurs services hospitaliers : MIM. Millard, Landouzy, Richelot et Marfan, et manquer, en terminant nos études médicales, de leur adresser nos respectueux remerciements.

Notre travail se divise naturellement en deux parties. La première, relative à l'étude des Arum ou plus exactement des Aroïdées indigènes, la seconde à l'étude des Actæa répulées toxiques ou médicamenteuses. Les Arum semblent bien devoir 
être définilivement rayés du nombre des plantes dignes d'emploi thérapeutique; par contre, ils intéressent, à plus d'un litre, la toxicologie et la médecine légale. Leur étude sera surtout une étude de botanique médico-légale.

Les Actées, au contraire. surtout l'espèce américaine, bien que loin de répondre à de multiples indications thérapeutiques, peuvent être des modificaleurs physiologiques dignes d'emploi dans certaines affections. Leur étude sera surtout une étude de botanique thérapeutique.

Dans l'exposé des caractères botaniquès, nous nous bornerons, volontairement, à ceux d'entre eux qui sont susceptibles d'intéresser directement la médecine. Certains de l'histoire botanique de ces plantes, non élucidés jusqu’ici, seront systématiquement passés sous silence, parce qu'ils ne touchent qu’à des questions de pure morphologie ou biologie végétale. L'étude chimique sera restreinte aux seuls principes actifs; à leur isolement et leur caractérisation.

L'expérimentation physiologique et les essais ont été poursuivis à la fois avec des extraits et avec des principes chimiquement définis. Les deux modes d'expérimentation se prêtent un mutuel concours et un contrôle simultané.

Contrairement à ce qui a été fait jusqu'ici pour l'Actée, nous avons tenu ì faire précéder les essais cliniques, au lit du malade, d’une étude physiologique expérimentale, portant sur un certain nombre de points particulièrement intéressants pour la pratique.

Nous avons insisté, avec quelque détail, sur diverses applications possibles des recherches d'histologie végétale à la médecine légale; c’est un sujet trop neuf et trop fécond, semble-t-il, pour ne pas être digne d'attention. 
$-4-$

Nous ne nous dissimulons pas les lacunes que présente notre travail. Tel qu’il est, il est le résultat de près de deux années de recherches; il nous paraît apporter quelques données précises sur des sujels nouveaux, ou jusqu’ici pleins de confusion. Ciest au jugement de ceux qui savent, par expérience, les difficultés (ne füt-ce que matérielles' inhérentes à semblables recherches, que nous livrons ces quelques résultats. 


\title{
ÉTUDES MÉDICALES
}

\author{
SUR LES \\ GENRES "ARUM" ET "ACTEA"
}

PREMIERE PARTIE

RECHERCHES MÉDICALES SUR LE GENRE ARUM

\author{
CHAPITRE PREMIER \\ ÉTUDE BOTANIQUE
}

A. - Arum maculatum L. et italicum MrLL.

Nous nous bornerons, volontairement, dans cette étude, à la description des caractères susteptibles d'intíresser le médecin: c'est dire que bien des points inléressants de l'histoire des Arum, søit au point de vue morphologique, soit au point de vue biologique, seront ici passés sous silence: nous nous réservons de les mettre en lumière dans un travail spécial.

Les caractères organographiques permettent une détermination précise et rapide de la plante.

Les caractères anatomiques et histologiques seront surtout de précieux documents pour la détermination de la plante en fragments, au cours d'une expertise toxicologique ou médico-légale. 


\section{MODE DE VÉGETATION DES Arum (1)}

Les Arum sont des plantes herbacées, vivaces, à lige entièrement souterraine, renflée en un lubercule, quiémet chaque annés,au printemps, une ou plusieurs pousses, protégées à la base par des écailles, et qui épanouissent quelques feuilles vertes, du milieu desquelles se dégage l'axe d'inflorescence. A la fin de l'été, cet axe persiste scul, après disparition des feuilles, et disparait lui-mème après la chute des fruits qu'il portait.

Chaque pousse nouvelle a donc sa vie réduite à la belle saison, et, au printemps suivant, ce sont une ou deux autres pousses vertes qui assureront l'assimilation et la fructification de la plante.

Tel est, daus ces grands traits, le mode de végétation des Arum, mais il imporle de préciser quelques détails.

A la germination, la plantule possède un axe hypocotylé, cylindrique, mais qui manifeste déjà sa tendance à se renfler; une racine pivotante, mais grêle, continue vers le bas cet axe; cette racine ne tarde pas à disparaitre, el désormais la plante n'aura plus que des radicelles adventives, de durée éphémère.

Dès la fin de la première année de végétation, l'axe hypocotylé s'est renflé en une tige souterraine qui constitue le tubercule, et qui, pendant au moins sa deuxième année de végétation, se borne à émettre des pousses vertes, sans fleurir. Les pousses vertes des sujets qui n'ont pas encore fleuri ont un axe tellement raccourci, que ses entre-nouds n'existent presque pas; clles sont protégées extérieurement par quelques feuilles, réduites à des gaines blanches, minces, enroulées sur elles-mêmes en spirale, sur toute leur hauteur, les extérieures bien plus courtes que les intérieures, ces dernières emboittées dans les premières; ces gaines se rabattent peu à peu vers le sol, pour livrer passage à une ou deux feuilles vertes, complètes, formées d'un pétiole et d'un limbe sagitté; leur pétiole se dilate ì sa base, et latéralement, en deux lames minces constiluant une gaine cnroulée sur elle-mème. La gaine de la feuille la

(1) Le mode de végétation des Arum n'a guère été étudiẻ,et d'une façon succincte, que par Irmisch. 
plus élevée abrite un bourgeon terminal, phus on moins développé, formé de feuilles complètes, avee pétiole et limbe, mais encore rudimentaires. A l'automne, la ou les feuilles vertes, épanuuies cette année par la plante, disparaissent, lo bourgeon qu'abritent leurs gaines attend au repos le printemps suivant.

Une importante réserve amylacée s'est accumulée, à la fin de l'été, dans le tubercule qui s'est ainsi considérablement rentlé.

Au printemps, le bourgeon terminal, jusqualors dormant, epanouit 2, parfois 3-4 feuilles vertes, complètes, de la gaine desquelles se degage l'axe d'inflorescence, la plante fleurit alors pour la première fois, et l'axe qui a fleuri a terminé son évolution. La plante, réduite jusqu'alors à un axe simple, va continuer sa végétation en se ramifiant.

L'avant-dernière feuille verte de l'axe qui fleurit porte ì son aisselle, abrité dans le fond de sa portion vaginale, un bourgeon encore rudimentaire, abrité par une première gaine, opposée à la feuille axillante. C'est ce bourgeon qui s'épanouira l'année suivante, en pousse feuillée et fleurie.

(Nous ne discuterons pas ici la question de savoir si les Arum ont une ramification mono ou sympodique. Une longue description de leur mode de ramification, pendant plusicurs années successives, nous entrainerait trop loin, et ne présenterait qu'un intérèt restreint, au point de vue strictement médical qui doit, seul, nous préoccuper ici.)

A l'aisselle des feuilles les plus inférieures, réduites ì leur portion vaginale, existent également des bourgeons dormants, plus ou moins développés, qui apparaissent libres à la surface du tubercule, après la disparition des gaines. Ce sont des bourgeons dormants de réserve, susceptibles de rester plusieurs années à l'état latent, prèts à remplacerle bourgeon terminal, si celui-ci venait à être détruil, et capables de se détacher du pied mère, pour ètre l'origine d'autant de pieds nouveaux.

Chez Arum maculatum, les petits bourgeons qui se détachent du tubercule prennent d'abord une forme allongée, sorte d'ébauche d'un rlizome horizontal. Tandis que la portion la plus âgée, pri- 
nilive nunt atlenante au tubereule, se flitrit, la portion la plus jeune se renfle légèrement; au niveau des cicatrices des premières écailles protectrices de ce bourgeon, émergent des radicelles advenlives, et déjà quelques bourgeons dormants.

Pendant sa premièreannce de végétation, ce bourgeon noimet guire qu'une fruille, pelite, à limbe obovale, très entier, quelquefois un peu allénué à la base. Lil forme de cesfeuilles présente: un grand interet pralique, a causede leur ressemblance aver celles de l'oseille, comme nous le dirons plus loin.) L'extrémité en voie de croissance horizontale du bourgeon perce, pour se fraver un passage, la base de la gaine de la première feuille'; elle ne porte, à la fin de cette première année de végétation, que trois petites écailles terminales, réduites ì une portion vaginale, et à un petit acumen en capuchon, constituant ainsi un bourgeon terminal dormant, qui ne continuera son érolution que l'année suivante.

Cette croissance horizontale du bourgeon, pendant la première année de sa vie indépendante, explique la direction horizontale si fréquente du tubercule.

Certains de ces bourgeons sont l'origine de la ou des pousses vertes accessoires, non fertiles, qui peuvent émerger du tubercule en même temps que la pousse principale fructifiante et fertile.

La base de ces pousses accessoires émet un cercle de racines adventives, et, à l'automne, se renfle en un petit tubercule, capable d'atteindre des dimensions plus ou moins considérables, arant de s’isoler du tubercule mère, pour mener une vie indépendante.

Les caractères ci-dessus indiqués s'appliquent indistinctement aux deux espèces indigènes d'Arum : A. maculatum et itaticum.

Commençons l'étude des caractères spéciaux à chaque espèce par celle de l'A. maculatum. 


\section{I. - ARUM MACULATUM L. 1753}

Syn. Arum vulgare. Tabern. 1588 (fid. Bauhin).

CAMER, 1588.

- LAM K. $1778-1794$.

\section{$1^{\circ}$ Caractères organographiques $(1)$.}

Le tubercule est charnu, renflé, de forme un peu variable, souvent irréguliere. La direction de ce tubercule est généralement horizontale ou oblique par rapport à la surface du sol, plus rarement tout à fait verticale.

A sa base, c'est-à-dire à sa partie la plus inférieure et la plus âgée, le tubercule présente une sorte de calotte friable nette lorsque l'examen est pratiqué à l'automne), constituée par la portion la plus vieille, qui se trouve isolée du reste de la masse par la formation au-dessus d'elle d'une assise tubéreuse. Au point de raccord de la portion âgée du tubercule et de la base d'implantation, médiocrement renflée, de la pousse de l'année, existe le plus souvent un śtranglement. Le tubercule émet sur ce point un cercle unique de radicelles adventives qui se détruisent, avant même qu'elles ne soient remplacées par un cercle de nouvelles radicelles, grêles, cylindriques, dépourvues de poils apparents, perçant, pour parvenir au dehors, les gaines qui entourent la base de la pousse.

Des cicatrices alternes, linéaires, arquées, indiquent, sur la partie àgée du tubercule, la place qu'occupaient les écailles et les feuilles des années précédentes.

Outre la ou les pousses vertes épanouies, le tubercule présente, en des points divers de sa surface, mais toujours à l'aisselle des cicatrices peu apparentes, laissées par les leuilles de l'année précédente, des bourgeons saillants, sous forme de petits boutons blanes, nacrés (et dont nous avons plus haut indiqué les rapports), la plupart

(1) L'A. maculatum ayant été figuré, avec plus ou moins de détails et d'exactitude, il est vrai, par de nombreux auteurs, nous avons préféré représenter en détail l'autre espèce indigène, l'A. italicum. (v. pl. 1.) 
de petite taille, et dont certains atteignent les dimensions d'une noisette; leur base se renfle alors en une masse, gorgée de rriserves féculentes, tandis que leur sommet s'entoure d'écailles blanchâtres ou rosées.

Les feuilles les plus externes, le plus souvent au nombre de deux́, sont reduites a de longues zaines incolores, formant de véritables squames; les feuilles les plus internes sont complètes, toutes alternes, imbriquées.

Ces dernières sont formées d'un pétiole, subcylindrique dans l'ensemble, rétréci au sommet, parcouru par de fines cannelures longitudinales, dilaté jusqu'à mi-hauteur en a levres membraneuses, dont l'une chevauche sur l'autre, formant par leur ensemble une large gaine, gaine atténuée prosressivement de la base au sommet.

Le limbe sagitté, deux fois moins long que le pétiole, hasté à la base, obovale, aigu au sommet, est lisse, luisant, entièrement vert ou parsemé de macules irrégulières d'un noir pourpré; on peut décrire le limbe comme formé de 3 lobes, le supérieur grand, les deux inférieurs petits, sous forme d'oreillettes deux fois moins longues que le lobe supérieur, courtes, descendantes, peu ou pas divariquées, à pointe plus ou moins aiguë. Il est parcouru par trois nerrures principales, saillantes à la face inférieure, une pour chaque lobe, raccordées ver's le haut par des anses; une nervure tout à fait latérale est très grêle.

Certains pieds ne portent qu'une seule feuille verte bien développée, on trouve alors, dans la gaine, une deuxiène feuille restée rudimentaire, surtout quant à son limbe. Certains autres pieds portent 3-4 feuilles vertes, c'est un fait rare; mais, assez souvent, 2 feuilles rudimentaires se trourent abritées dans la gaine de la deuxième feuille verte normale.

La feuille inférieure ou extérieure enveloppe, dans sa portion vaçinale, haute et fermée, la feuille supérieure ou intérieure, qui enveloppe elle-mème dans sa portion raginale l'axe d'inflorescence.

Quel que soit le nombre des feuilles, c'est à l'aisselle de la gaine de l'avant-dernière feuille que se trouve l'are d'inflorescence formant 
la terminaison de la pousse feuillée. Son pédoncule scape est plus court que le pétiole ou l'égalant à peine, cylindrique, vert, on parfois tacheté comme les feuilles, strié longitudinalement, porteur d'une large bractée (spathe), opposice it la derniere feuille, obovale dans l'ensemble, allénuée au sommet, it insertion oblique, curouléc sur elle-même en cornet, ovoïde et ventrue à la base, puis hrusquement rétrécie, puis dilatée à nouveau et progressivement en longue pointe, avant son épanouissement. Lor's de l'anthèse, lit portion supérieure seule de la spathe s'étale en un limbe, formant cornet largement ouvert au-dessus de la portion rétrécie qui demeure

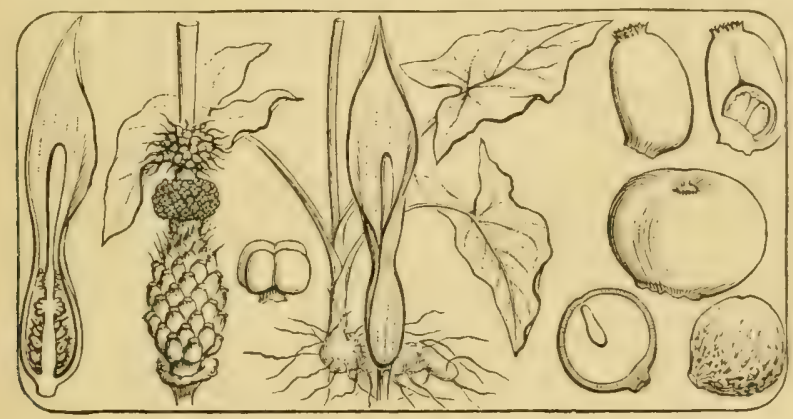

Fig. 1. Arum maculatum L. - Plante entière fleurie. - Inflorescence cn c. longit. - Base de l'infloresc. - Anthère. - Ovaire entier ct en c. longit. Fruit mûr. - Graine entière et en coupe.

enroulée, d'un vert jaunâtre, pàle, uniforme, ou hien maculée de noir, parfois simplement bordée de pourpre sale, parcourue par un très grand nombre de nervures longitudinales, parallèles, reliées par des anastomoses secondaires transversales ou obliques, le tout formant un réseau fort élégant, saillant surtout à la face interne.

Au-dessus de l'insertion de cette bractée, l'axe d'inllorescence se prolonge, sous forme d'une baguette cylindrique spadicel, moitié moins longue que la spathe, progressirement atténuée vers le haut, finalement terminće par une massue cylindrique, allongée, à sommet obtus.

Le spadice porte des fleurs apérian thées, unisexuées, les inférieures femelles, les supérieures mâles, et des fleurs stériles.

Presque immédiatement au-dessus de l'insertion de la spathe, 
šinsèrent sur le spadice une trentaine de fieurs fomelles, sessiles, réduites à un ovaire uniloculaire, d'un vert pâle, parfois un peu pourpre à l'extrême sommet, ovoïde dans l'ensemble, renflé rers le haut et du côté externe, à paroi molle, épaissie progressivement vers le haut, surtout du côté interne, à sommet obtus, portant sur le côté externe un bouquet de courtes papilles stigmatiques, qui limitent une surface visqueuse, "n continuitéavec un pertuis obturé par un bouchon mucilagineux, et qui conduit dans la cavité de l'ovaire. Un unique placenta, situé le long de la paroi inlerne, porte, sur les deux tiers inférieurs de sa hauteur, un petit nombre d’ovules, quelques-uns insérés presque à la base, très incomplètement anatropes, les inférieurs obliquement ascendants, les moyens sensiblement horizontaux, les supérieurs obliquement descendants, à funicule très court, entourés à leur base d'une sorte de frange, périfuniculaire, émanant du placenta. Cette frange est formée par de nombreux poils pluricellulaires, simples; ces poils émanent de toute la surface du placenta, et même de la base des funicules; c'est Parlatore qui a, le premier, attiré l'attention sur ces formations, dont l'existence est très générale chez les Aroïdées.

Le nucelle des Arum est remarquable en ce que les cellules qui constituent sa partie supérieure sont oroïdes, libres dans un liquide granuleux, et munies chacune d'un novau fusiforme, particularités signalées, il y a longtemps, par TulasNe (1).

Au-dessus de ces fleurs femelles fertiles, se trouvent quelques rangées circulaires d'organes avortés, considérés comme des fleurs femelles rudimentaires, à base lisse, à sommet convexe, garni de saillies, du sommet duquel se détache un style court, atténué, el finalement recourbé en croc vers le bas.

Un manchon de fleurs mâles fertiles succède à cette bague de tleurs femelles stériles; l'inflorescence mâle est $3-4$ fois plus courte que l'inflorescence femelle. Ces fleurs mûles, nombreuses, serrées les unes contre les autres, et mème déplacées par pression réciproque, sont réduites chacune à une étamine, implantée comme

(1) Ann. des Sc, natur. Bot., 4 sćrie, t. IV, p. 101, 1855. 
un clousur l'axe du spadiee. Celle-ciesl libre, ì filel courl, insérépar une portion nolable de sil longueur sur laaxe du spadice, à anthère obtuse, quelque peu allinuéc à la base, formée primilivanenl de a logettes, mais ne présentant plus, à l'âge adulte, que deux loges latérales, déhiscentes latéralement par une fente longitudinale.

Un court intervalle sépare ces fleurs d'une seconde bague de fleurs stériles, très analogues aux fleurs stériles de la base, moins dilatées que celles-ci, progressivement atténuées en une sorte de style grêle, allongé, non incurvé, dirigé vers le bas.

Le manche de la massue qui vient ensuite, d'une couleurjaune pìle, présente quelques petits accidents de surface, oì l’on a voulu voil autant d'ébauches avortées de fleurs màles. La massue est d'une teinte violette, égale au manche, parfois plus courte ou plus longue que lui.

Aux ovaires succèdent des baies vertes, puis rouges à maturité (vers la fin de l'été), de forme variable, déterminée le plus souvent par la compression exercée extérieurement par les fleurs voisines, et par la distension intérieure due à l'accroissement des graines, à péricarpe charnu, succulent, plus épais au sommet, renfermant un petit nombre de graines (parfois une seule) ovoüdes, quelque peu irrégulières, çà et là fovéolées à la surface, ì hile punctiforme. Le funicule de l'ovule persiste, sous forme d'un mince cordon vasculaire; sous des téguments épais, elles renferment un albumen abondant, charnu, qui loge vers son sommet un petit embryon cylindrique, monocotylédoné.

Dans la cavité du fruit, par conséquent autour des graines, se trouve une sorte de pulpe, gélatineuse si le fruit est placé à l’humidité. Cette sorte de masse gélatineuse est due à la gélification des poils placentaires et funiculaires, dont nous avons signalé la présence dans l'ovaire.

Les dimensions moyennes de la plante sont plante adulte fleurie' :

Tubercule $\left\{\begin{array}{l}\text { longueur } \ldots \ldots \ldots \ldots \ldots \ldots \ldots \\ \text { largeur maxima } 4.5 \mathrm{~cm} \\ 2-2,5 \mathrm{~cm} .\end{array}\right.$

Feuille.

Pétiole longueur........... $20^{\mathrm{cm}}$ 


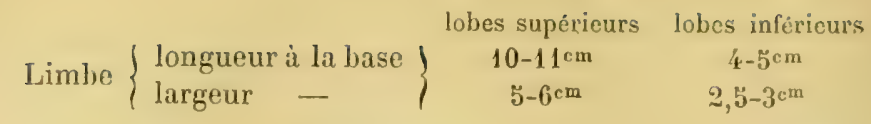

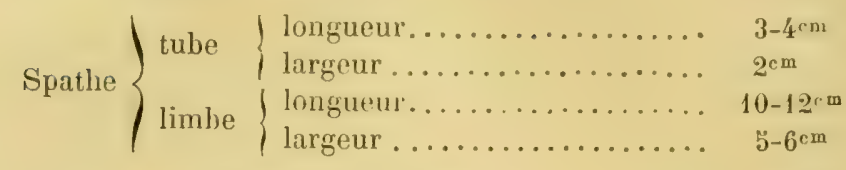

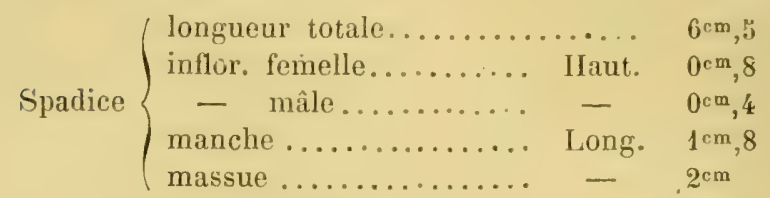

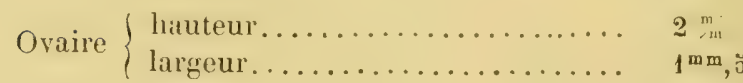

Ovule................... Haut. $0^{\mathrm{mm}, 6}$

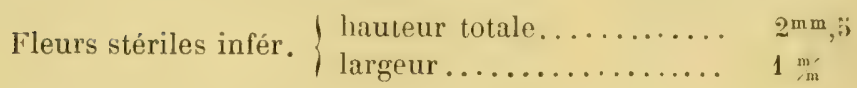

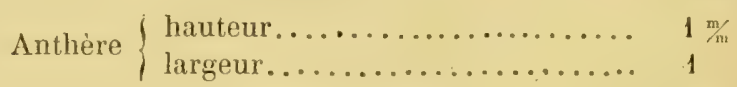

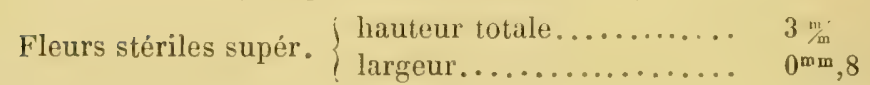

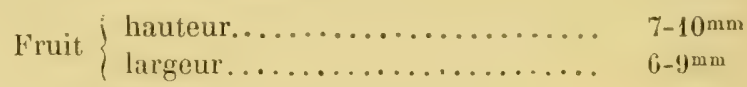

On connaît diverses variétés et formes d'Arum maculatum, qui ont été élevées par quelques auteurs, et surtout par Scrotr, au rang d'espèces distinctes.

Les caractères réputés spécifiques sont tirés de la coloration de la spathe, des dimensions de la massue du spadice et de son manche En réalité, il s'agit d'une espèce quelque peu sujette à variation. Nous n'avons à signaler ici que les variétés susceptibles d'être rencontrées dans notre pays, et par suite dignes d'intérèt pour le médecin.

Le nom spécifique de maculatum n'est pas justifié d'une façon générale. De nombreuses plantes de cette espèce, non maculées, croissent molées aux plantes franchement maculées; dans certaines régions même, le nombre des pieds non maculés est plus considé- 
rable que celui des pieds maculés. On peut done distinguer deux variétés :

$1^{\text {ro }} v a r$. vulgaris immaculata, la plus répandue (Arum immaculatum Sспотт. - A. Zelebori, Scirotт). Le limbe foliaire y est d'un vert uniforme, la spathe a un tube à fond blanc, à zone pourprée médiane, un limbe vert à la face interne, d'un pourpre sale au bord, une massue d'un vert pâle ou jaunâtre.

$2^{\mathrm{e}}$ var. vulgaris maculata. - Le limbe foliaire est, à sa face supérieure, irrégulierement parsemé de laches noires ou pourprées, la spathe a un tube, avec zone pourprée supérieure à sa face interne, un limbe vert, parsemé çà et là de taches irrégulières, de même collleur que celles de la feuille.

Distrib. GÉOGRAPHiQUe. - Arum maculatum est commun dans tous les lieux ombragés de l'Europe moyenne et méridionale. Bornons-nous à quelques indications sur sa distribution géographique en France.

C'est à tort que l'on a indiqué parfois (BAUTIEn) A. maculatum comme répandu par toute la France. Il est très rare à Montpel lier (DE CAND.); il existe dans les parties montueuses de la Haute-Garonne, du Tarn, du Gard. En Auvergne, il se rencontre partout dans les prairies et les montagnes jusqu'à 1.000 mètres (LECOQ), dans les Pyrénées avec une fréquence plus ou moins grande, selon les localités (LAPE Y ROUzE, ZETTERST., DulAC), dans les Bouches-du-Rhòne, l'Aveyron, le Lot; rare dans le Forez; dans la Seine-Inférieure, la Seine, Seine-et-Oise, la Cóted'Or, Maine-et-Loire, il domine à la presque exclusion de l'A. italicum; il existe seul dans Meurthe-et-Moselle, la Meuse, le Dauphiné, le Doubs, l'Alsace, le Jura, le Morbihan. Dans toute la région de l'Ouest, l'A. italicum le remplace presque complètement.

\section{$2^{\circ}$ Caractères anatomiques.}

L'étude systématique de la structure anatomique et histologique des Arum indigènes n'a pas encore été faite jusqu'à ce jour.

On ne trouve, sur ce sujet, que quelques renseignements sur des points spéciaux, épars dans les mémoires de Van Tiegnem, de Dalitzch, Garcin, Courchet .

L'importance spéciale que présente, au point de vue médico-légal, 


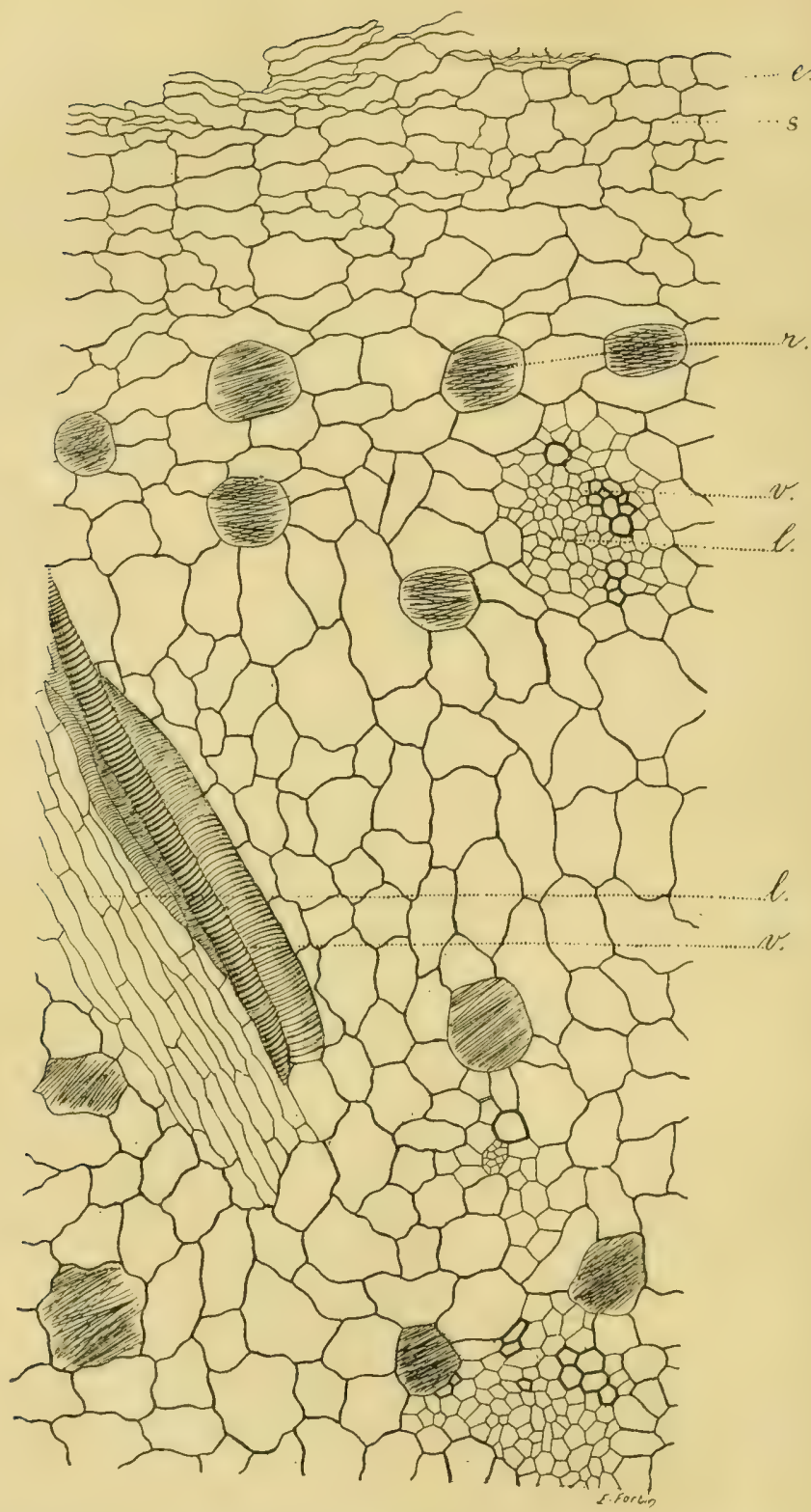

Figr. 2. Arum maculalum L. (Gross. 120/1). - Tubercule (portion âgée) raphides.
. $l$ faisceaux libériens, $v$ vaisseaux ligneux, $v$ cellules it
ingé 
la détermination histologirgue des organes entiexs ou fragmentés des Arum justifient les détails qui suivent.

Tutbercule (portion âgib. Epiderme partout exfolii par une assise subéreuse brune, différenciée à une faible profondeur dans le parenchỵme périphérigur, assise formé d'éliments aplatis, peu sinueux, régulièrement alignés en files radiales (.j-6 assises concentriques environ), à parois minces, peu colorables par le vert d'iode. Des plarfues de parenchyme morl, a éléments vides, arlhèrent par endroits à Ia face externe de l'assise subéreuse.

La masse du tubercule est formée d'un parenchyme homogène, à éléments ípolygonaux dans lit zone centrale, quelque peu aplatis dans la zone périphérique) oboviles, assez réguliers, à paroisminces, sans méats.

Le tubercule, examiné à la première période de végétation, se montre privé de tout contenu mucilagineux, semblable ì celui que l'on observe, à la période de repos (V. Arum itaticum, p. 34), privé aussi d'amidon.

Des éléments, de taille un peu plus considérable, groupés avec plus d'abondance vers la périphérie, sont abondamment pourvus de paquets de raphides.

Faisceaux cribro-vasculaires nombreux, ramifiés dans toute la masse du tubereule et dius toutes les directions, à éléments vasculaires placés en dedans, ou parfois à l'intérieur d'un faisceau cribreux, uniquement formés d'éléments spiralés, non déroulables, faiblement lignifiés, à section polygonale, de longueur médiocre, et terminés en pointe aux deux bouts.

Ces faisceaux émettent des branches simples, qui se réunissent à d'autres faisceaux par de fréquentes anastomoses, ou qui se rendent aux feuilles et à l'axe d'inflorescence.

Le tubercule est privé de tout élément de soutien.

Raclicelle adventive (struct. primaire).

L'écorce se divise naturellement en deux portions très distinctes; a l'externe, $b$ l'interne, beaucoup plus large que l'externe. La portion externe $a$ se divise elle-même en trois zones :

$1^{\circ}$ Zone externe à limites bien nettes, surtout sur les coupes colo- 


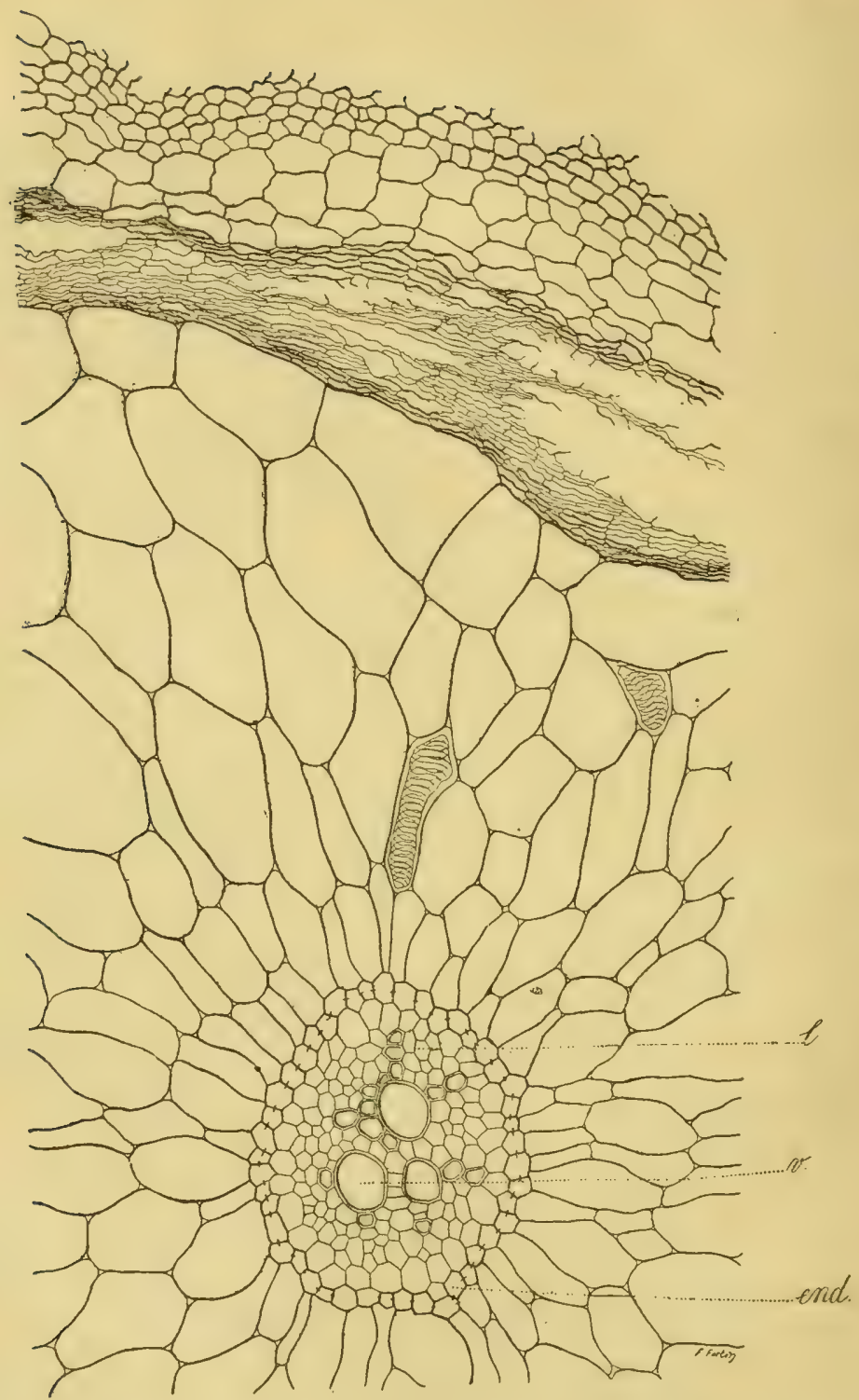

Fig. 3. Arum maculatum L. (Gross. 120/1). - Radicelle (str. prim.) C. trans. $-l$ faisceaux libériens, $v$ vaisseaux, end. endoderme.

rées, formée de deux ou trois assises de cellules mortes (l'assise la plus externe désagrégée par endroits, à éléments plus ou moins 
saillants vers l'extérieur), fortement colorables (vert d'iode), lignifiées.

$2^{\circ}$ Zone moyenne, à peine colorable, à éléments assez régulier, la plupart aplatis, avec rares méats, à parois minces.

$3^{\circ}$ Zone interne ì éléments morts, tous aplatis et tilssés tangentiellement, à parois minces, à peine colorables; de larges lacunes aérifères, à section ovalaire, allongées tangentiellement, résultent de l'écartement et des déchirures subis par les assises qui les bordent.

La portion interne $b$ est uniformément formée de grands éléments, de dimensions régulièrement décroissantes, et plus arrondis à mesure que l'on se rapproche du centre, assez réguliers, la plupart allongés et alignés dans le sens radial, séparés par quelques petits méats, à parois peu épaisses, colorables (carmin); les éléments les plus internes dessinent, d'une façon plus ou moins nette, autour du cylindre central, deux ou trois cercles concentriques.

Çà et lì, quelques cellules, restées de petites dimensions, ont été écrasées, lors de l'accroissement en épaisseur de cette zone, leur paroi s'est détachée de celle des cellules qui les entourent, et apparait, avec d'élégantes spirales d'épaississement, dues sans doute à la torsion de la membrane sur elle-même.

Endoderme (encl.) très net, à parois minces, avec cadres d'épaississement bien visibles sur les parois radiales.

Les éléments de l'assise péricyclique ne se distinguent que par leur taille un peu plus considérable de ceux qui constituent la masse conjonctive du cylindre central.

Les faisceaux libériens (I), cribreux, sont à peine distincts (sur la coupe transvers.) du parenchyme ambiant, leurs cellules ont une section sensiblement hexagonale, des parois brillantes.

Ces faisceaux cribreux sont séparés des faisceaux vasculaires par des cellules, à parois plus minces, identiques à celles qui occupent la région médullaire (ces dernières cependant un peu plus larges).

Les faisceaux vasculaires ligneux, au nombre de quatre, sont formés de vaisseaux dont le diamètre augmente progressivement vers le centre, vaisseaux spiralés, bien lignifiés, les plus àgés 
(périphériques) ì section faiblement polygonale, les plus jeunes (centraux) à section circulaire.

Il arrive, assez souvent, que le raisseau le plus interne d'un faisceau grandisse plus rapidement que le vaisseau le plus interne du fiasceatu roisin, il empiète alor's sur ce faisceau, et forme la pointe d'un $\mathrm{V}$, dont les deux faisceaux constituent les branches.

Feritlle $n^{\circ} 1$ (pied âgé). Pétiole.

C. initicle (à mi-hauteur de la portion vaginale du péliole) en fer à cheval, à branches aiguës, l'une chevauchant sur l'autre; faisceaux à bois interne, sur deux ares concentriques dans la région

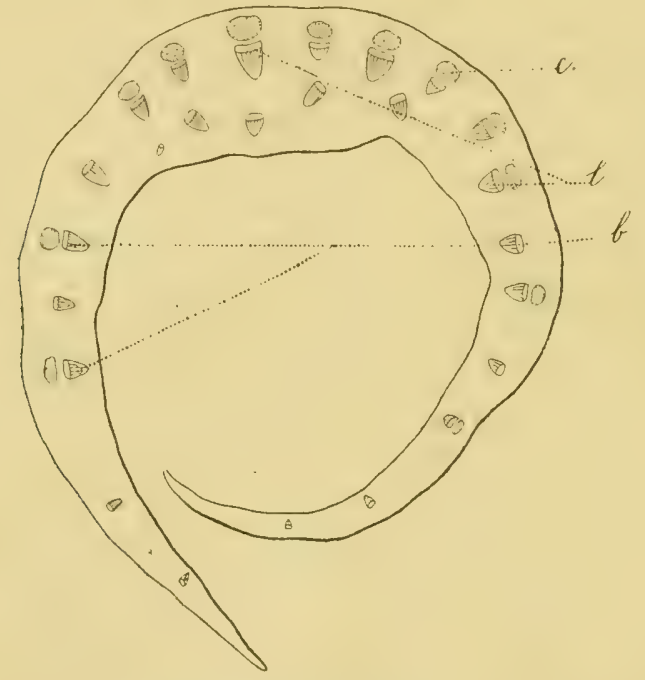

Fig. 4. Arum maculatum L. (Gross. 8/1). - Pétiole, c. initiale). - $l$ libr., $b$ bois, $c$ collenchyme.

médiane renflée, sur un seul dans la portion amincie qui constitue la gaine; vingt faisceaux sur l'are externe, cinq seulement sur l'arc interne.

C. médiane (à la pointe de la gaine) subelliptique, quelque peu aplatie sur la fare interne; faisceaux sur deux ares concentricues, irréguliers, ouverts du côté interne; treize faisceaux sur l'arc externe, dix faisceaux sur l'are interne, trois faisceaux centraux, à bois interne. 


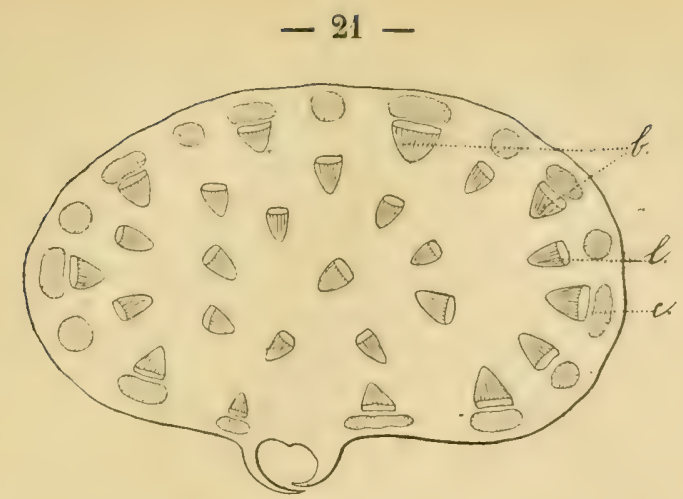

Fig. 5. Arum maculatum L. (Gross. 8/1). - Pétiole, c. médiane.

C. terminale ou caractéristique (à la naissance du limbe) obconcave (vers le côté interne), arec deux larges ailes qui commencent à se dessiner sous forme d'angles latéraux aplatis; les treize faisceaux de la médiane s'y retrouvent, quelques-uns en train de se

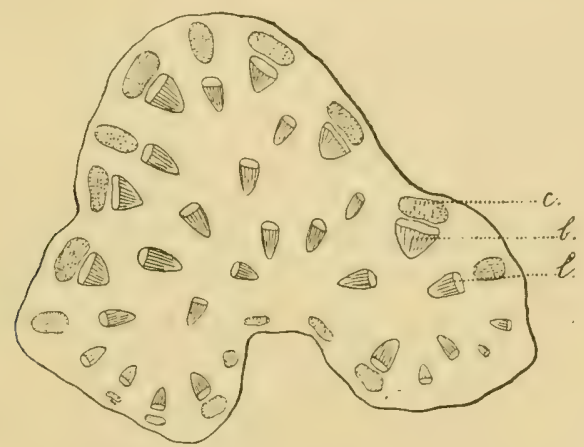

Fig. 6. Arum maculatum L. (Gross. 8/1). - Pétiole, c. terminale.

diviser pour fournir aux nervures du limbe, tous à orientation normale; les dix faisceaux de l'are interne se sont alternativement rapprochés el écartés du centre; un des trois faisceaux centraux a disparu.

Nervure médiane du limbe (G. au milieu) convexo-concave, à dépression supérieure médiane; deux arcs de faisceaux, ouverts vers la face supérieure: l'un, de huit gros faisceaux, situé près de la face inférieure; l'autre, formé de bien plus petits faisceaux, situé près de la face supérieure, tous à bois supérieur; deux faisceaux cen- 
tranx ncrupent, superposés l'un at l'autre, le plan de symétrie, tournant leur bois vers la face supérieure.

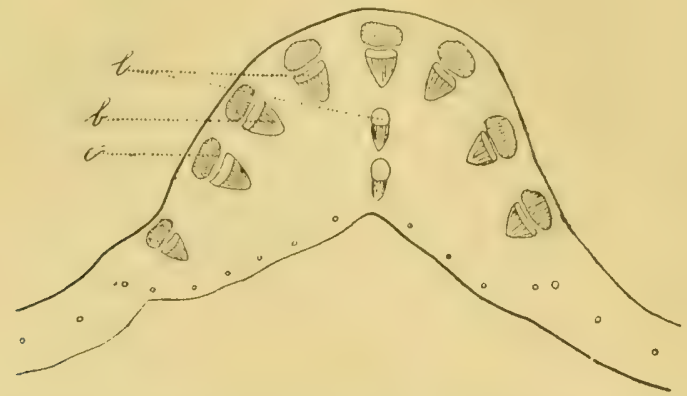

Fig. 7. Arum maculatum L. (Gross. 8/0). - Nervure médiane du limbe, c. au milieu.

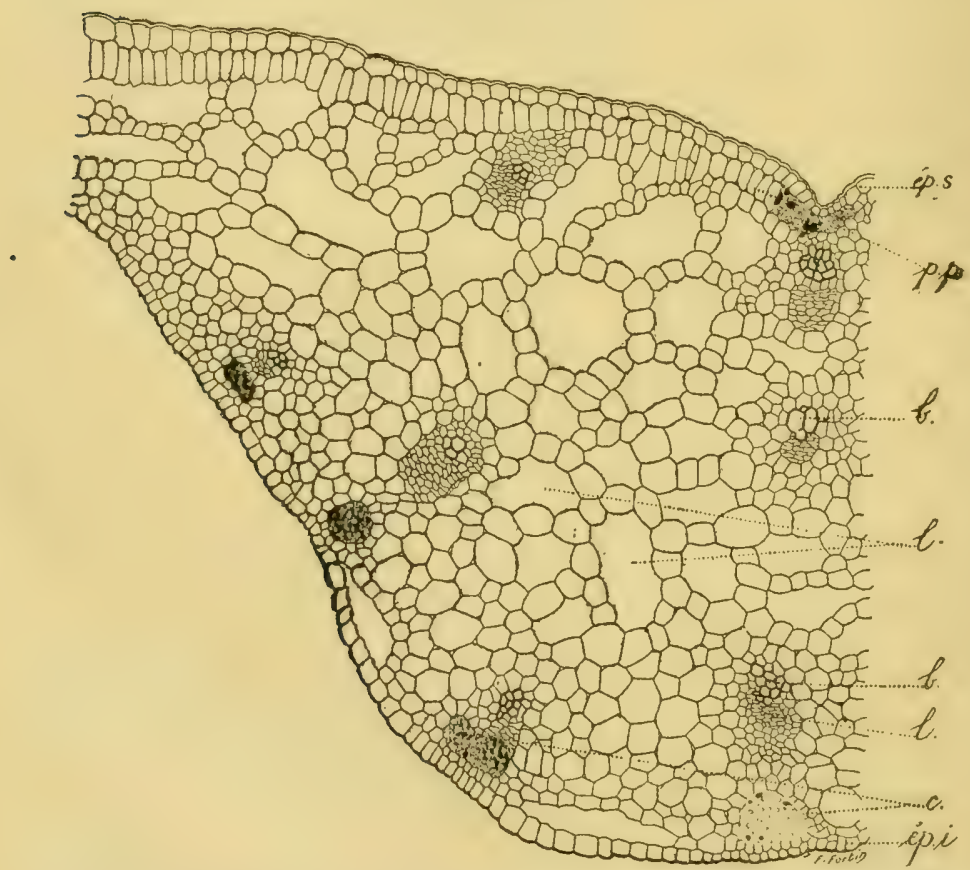

Fig. 8. Arum maculatum L. (Gross. 40/1). - Nervure médiane du limbe, c. au milicu. - ép s épiderme, $p p$ parenchyme palissadique, $b$ bois, $l$ liber., $c$ collenchyme, épi épiderme inférieur.

La coupe transiersale à ces divers niveaux est toujours légèrement onduleuse; chaque ondulation est presqu régulièrement 
déterminée par la saillie d'un massif de collenchyme; la plupart de ces massifs sont placés au dos d'un des faisceaux de l'are externe.

Au-dessus de la portion vaginale (e. médiane, les faisceaux périphériques forment un cercle presque complet; mais les faisceaux centraux sont disposés sur une courbe, ouverte en haut du côté interne (cóté de la suture de la gaine); on en trouve trois autres, orientés, avec plus ou moins de symétrie, de part et d'autre du plan médian.

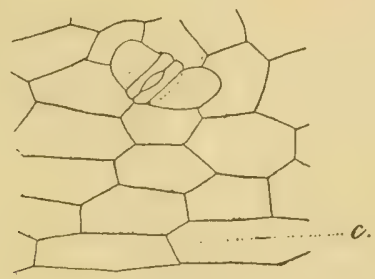

Fig. 9. Arum maculatum L. (Gross. 120/1). - Epiderme du pétiole. $-c$ portion superposée aux faisceaux de collenchymo.

Tous les faisceaux cribro-vasculaires périphériques sont superposés à autant de faisceaux de collenchyme; mais ils sont de deux sortes, et alternent régulièrement. Les faisceaux les plus externes ont leur système vasculaire réduit à un paquet de faisceaux étroits, séparé du faisceau du collenchyme correspondant seulement par une rangée de larges cellules amylifères. Les faisceaux plus internes sont séparés du faisceau du collenchyme par 4 -̋̈ rangées de larges cellules vides; chez ces (lerniers, la liaison des faisceaux de collenchyme arec les faisceaux cribro-rasculaires est donc beaucoup moins intime.

Tous les faisceaux périphériques ou centraux possèdent : $1^{\circ}$ un arc de larges cellules libériennes, qui ont de chaque cuté une file de cellules pleines de latex; $2^{\circ}$ un faisceau de cellules cribreuses plus étroites; $3^{\circ}$ des raisseaux /en nombre très réduit pour les faisceaux périphériques) serrés les uns contre les autres, dont les plus internes (1-2) sont de plus grand liamìtre, à paroi rarement résorbée. Ces vaisseaux sont séparés les uns des autres et entourés de nombreuses cellules, étroites d'abord, puis de plus en plus 
larges, pleines de chlorophylle, et passant finalement aux cellules du parenchyme.

L'épiderme est formée de cellules ì section transversale obqua-

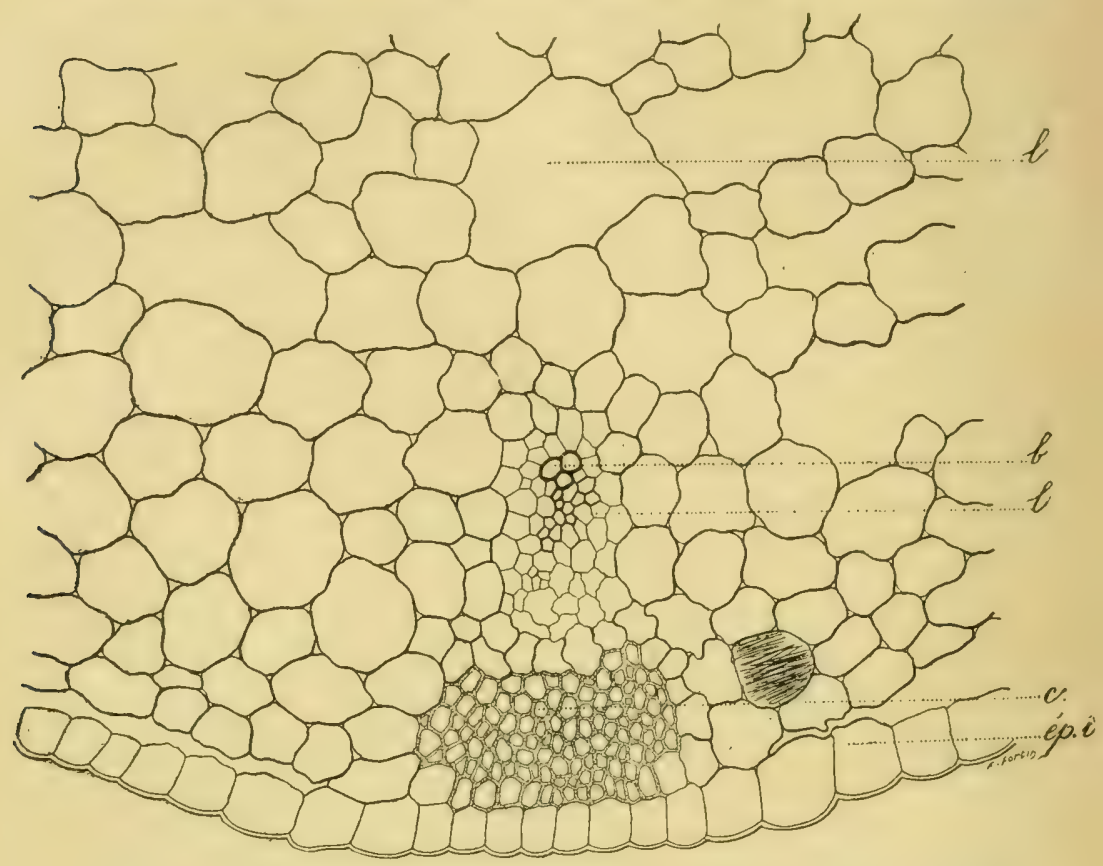

Fig. 10. Arum maculatum L. (Gross. 120/1). - Portion latéro-inférieure de la figure 8 .

drangulaire (celles superposées aux faisceaux du collenchyme plus petites), à paroi mince, plus épaisse sur la face externe, se prolongeant en crêtes minuscules (visibles seulement it un fort grossissement), que recouvre la cuticule.

Le parenchyme est formé d'éléments à parois minces, polyédriques, serrés, verts, avec nombreux méats dans les couches périphériques, plus arrondis, incolores, un peu onduleux, avec de grandes lacunes dans la région centrale el supérieure jou intérieure).

Limbe. - Epiderme à élémentspolygonaux oufaiblement ondulés, allongés au-dessus du trajet des nervures, à cuticule faiblement striée. 
Stomates présents sur les deux fices, mais surtuut nombreux it la face inférieure, obovales dans l'ensemble, accompagnés de 2 plus rarement de 3 it 4 , par suite du cloisonnement des cellules

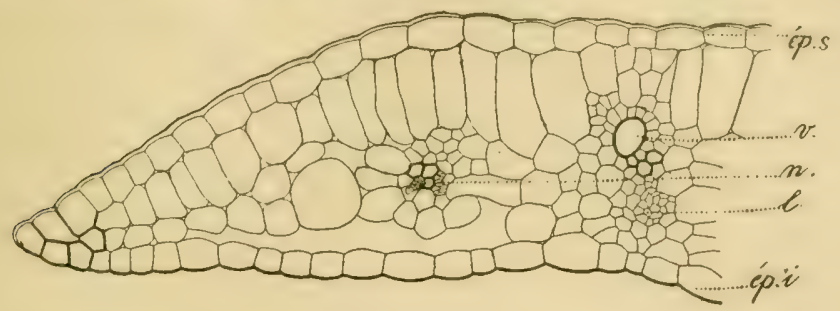

Fig. 11. Arum maculatum L. (Gross. 120/1.) - Limbe foliaire, c. transvers., portion marginale. - $n$ nervure.

annexes primitives) cellules annexes latérales, semi-lunaires, convexo-concaves; la face externe des cellules stomatiques surplombe, en se rabattant en dedans, l'ostiole; leurs deux lèvres externes, nettement et finement striées, limitent ainsi une petite chambre sus-ostiolaire.

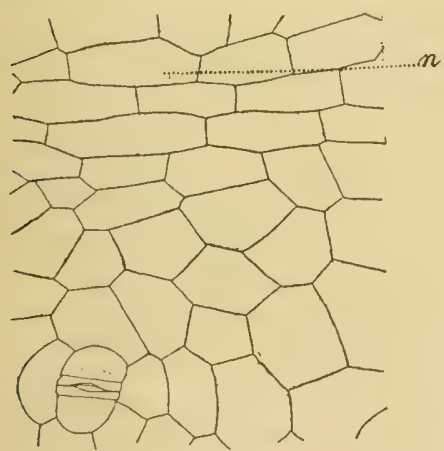

Fig. 12. Arum maculatum.

(Gross. 120/1). - Epiderme supérieur

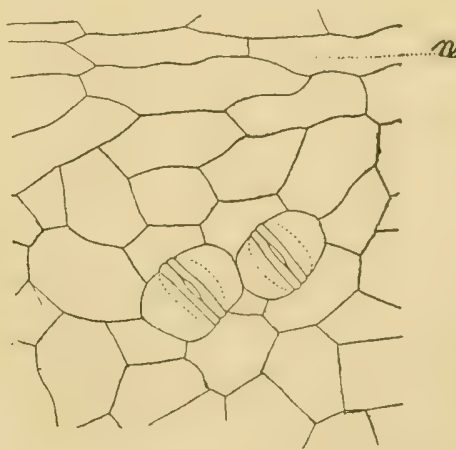

Fig. 13. Arum maculatum (Gr. 120/1). Epiderme inférieur du limbe. $n$ portion superposée à uno nervurc.

Mésophylle unifacial ; une seule assise de parenchyme en palissade à la face supérieure, çà et lì clifférencièe en éléments raphidifères, mésophylle inférieur lacuneux, à éléments onduleux, la plupart aplatis tangentiellement, limitant des chambres sousstomatiques. 
A l'extrime bord du limbe, les cellules épilermiques et quelques eléments du parenchyme épaississent leurs parois internes, pour servir d'éléments de soutien.

Nervures isolées dans le mésophylle, à peu près à mi-distance des deux faces; leurs éléments vasculaires sont réduits à fuelques éléments spiralés, toules sont entourées d'une gaine de parenchyme condensé, à ćléments petits, qui, pour les nervures principales, s'étend jusqu'au contact des deux faces de la feuille.

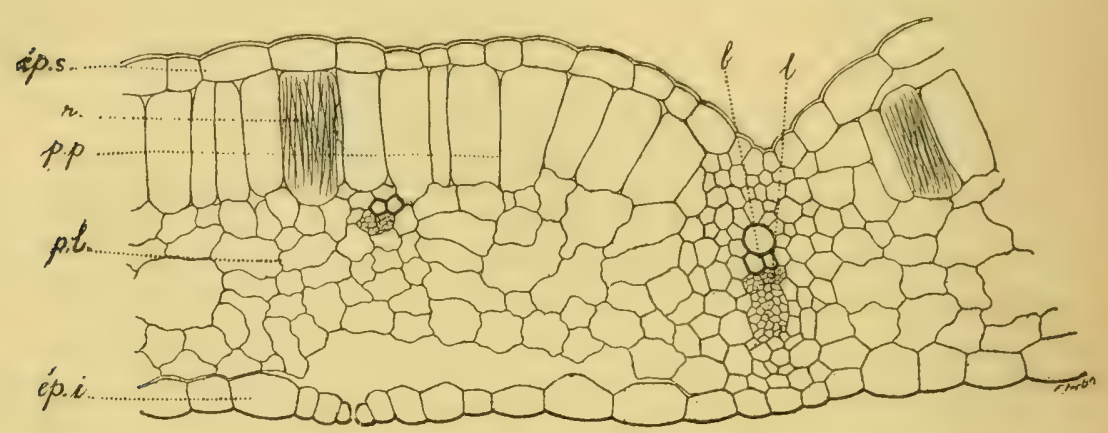

Fig. 14. Arum maculatum L. (Gross. 120/1). - Limbe foliaire (c. transvers.). Portion voisine de la nervure médiane; $p p$ parenchyme palissadique, $p l$ parenchyme lacuneux, $r$ éléments raphidiféres.

Les éléments raphidifères sont nettement visibles à l'œil nu, sous forme de minuscules traits brillants, à la face inférieure de la feuille. Leur situation, immédiatement au-dessous de l'épiderme, permet de les voir par transparence.

La presque totalité des cellules du parenchyme en palissade présentent, à leur intérieur, des masses de substance mucilagineuse, dignes de fixer l'attention. Ce sont des masses vermoïdes, occupant lo centre des cellules, parfois avec étranglement médian. Ces masses coagulables par l'alcool et le formol, colorables en violet par l'action successive du vert d'iode et du carmin aluné, ne sont passensiblement altaquées par un séjour modéré dans les hypochlorites alcalins, el les coupes, débarrassées par l’action de ces réatctifs des substances protéiques, les présentent avec une netteté parfaile. Les cellules du parenchyme lacuneux renferment aussi, mais d'une ficcon moins uniforme, parfois sous forme de simples granules 


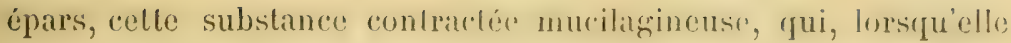
y est abondante, y revêt plutôt la forme de masses arrondies.

Dans l'épiderme, particulièremenl l'épid. inférieur, charpue cellule, y compris les cellules stomatiques et les cellules annexes, contient aussi une masse centrale, contractée, de la même substance.

C'est ce contenu mucilagineux qui donne au suc, extrail des parties vertes, sa consistance gommeuse. Les Arum contiennent donc des cellules mucifères, d'un type très spécial, el presque identiques à celles des feuilles de l'If (T'axus baccata). Nous reparlerons de l'intérét qu'elles présentent pou les recherches histologiques médico-légales.

Axe d'infloressence. Pútoncule tu spuntire, elliptique, un peu irrógulier à la coupe transversale.

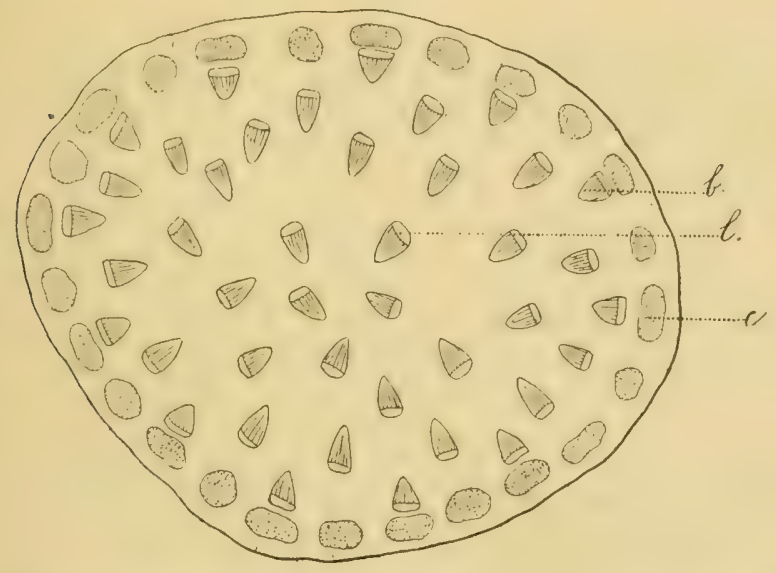

Fig. 15. Arum maculatum L. (Gross. 10/1). - Pédoncule du spadicc. (c. à mi-bauteur).

Epiderme à éléments rectangulaires (en c. transvers.) à parois minces, à cuticule striée.

Le parenchyme péliphérique et central est homogène, à éléments réguliers, obovales ou polygonaux, separés par des méats, it parois minces, plus petits et lassés à la péripherie, non parcourus par des lacunes.

Nombreux faisceaux périphériques de collenchyme, la plupart 
superposés aux faisceaux cribro-vasculaires; les élements collenchymateux ont des parois relativement minces, fort épaissies aux angles.

Faisceaux cribro-vasculaires, ovales allongés (en c.), à éléments rasculaires spiralés, la plupart de petit calibre. Un cercle périphérique régulier contient (c. pratiquée à mi-hauteur de l'organe) 12 faisceaux à peu près; un autre cercle, sous-jacent, alterne à peu près régulièrement avec le précédent; 16 faisceaux internes se répartissent avec une certaine irrégularité, de telle sorte que l'ensenıble des faisceaux est à peu près orienté symétriquement par rapport à l'axe de l'organe.

Syathe. - L'épiderme externe a des éléments polygonaux jus de face) ou quelque peu onduleux, présentant une tendance générale à

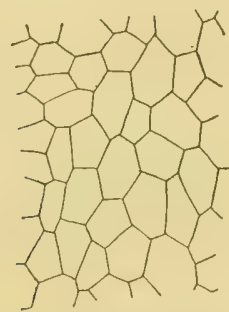
Fig. 16. Arum maculatum L.
Gross. 120/1). - Epiderme inter

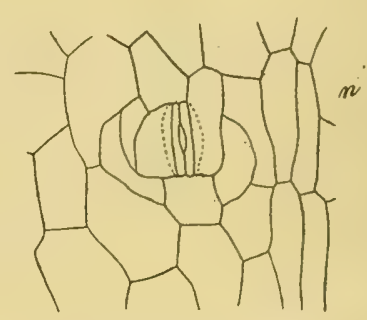

Fig. 17. Arum maculatum L. Spathe

l'allongement suivant la longueur de l'organe, très nette dans les portions superposées aux nervures. Les stomates y sont rares.

L'épiderme interne a des éléments polygonaux (vus de face), à section transversale obquadrangulaire, légèrement saillants en papilles ì la face interne, à parois minces, il est totalement dépourvu de stomates.

Sur les divers organes des Arum, il est facile de distinguer, même à l'ceil nu, surtout après disparition de la chlorophylle, par un long séjour dans une solution conservatrice (formol par ex.), par transparence ou par reflexion, d'innombrables petits points brunâtres. 
Chatque point correspond it une cellule épidermique ou plus sourent hypodermirque, a contenu brun foncé plus ou moins abondint. Ce contenu est formé par une résine, que l'on retrouve à l'analyse chimique, en très minime quantité.

Les mêmes cellules résiniferess existent dans lat profonteur des parenchrmes, mais arec moins d'abondance. Lorsigu'il si trouve en petile quintite dans la cellule considérie, le contemu se présente sous la forme de sphérules síparées, milis, en général, il occupe toule la cellule, et la rend totalement opaque.

Ces cellules se rencontrent sur: les feuilles incomplètes de la base des pousses rertes, les pétioles (avee une abondance particulière sur la portion vaginalej, la spathe iparliculirrement at la base et it la face interne, la portion supérieure du pédoncule du spadice, la base de celui-ci, les ovaires; par contre, elles font presque defaut sur le limbe foliaire, la base de l'axe d"infloreseence, abrité dans les gaines foliaires elles n'y apparaissent qu'après la chute des feuilles', es fleurs mâles et stériles, le manche et la massue du spadice.

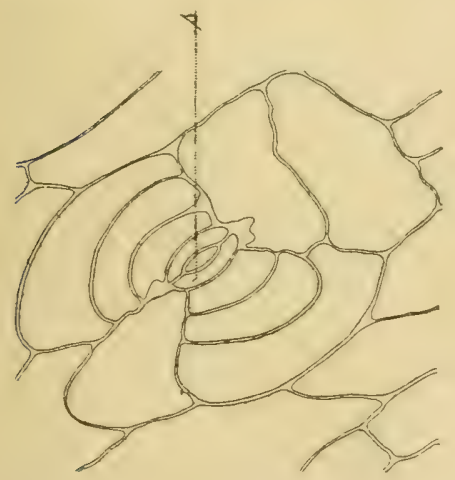

Fig. 18. Arum maculatum L. (Gross. 120/1). - Epicarpe. $s$ stomate.

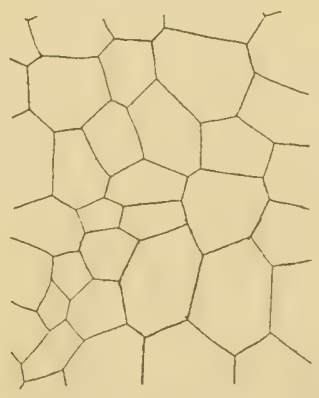

Fig. 18 bis. Arum maculatum L. (Gross. 120/1). - Endocarpe.

Sans nous prénceuper ici de leur signification biologiqur, nous ne mentionmons ces rellules que parce que, à priori, leur contenu résineux pourrait étre regardé comme un des principes actifs de la plante. L'analyse chimique el les essais physiologiques montrent d'ailleurs que cette hypothèse n'est pas justifiée. 
Fruit. - Les caractères histologiques du péricarpe sont très analogues à ceux du péricarpe de l'A. italicum, que nous étudierons plus loin avec détail.

L'épicarpe a cependant des éléments de plus grande dimension, plus onduleux, plus arqués au voisinage des stomales, ces derniers sont sensiblement sur le mème plan „que les cellules épidermiques.

Graine. - Ses caractères sont trop analogues à ceux de l'A. italicum, que nous décrivons et figurons plus loin, pour qu'il soit nécessaire d'y insister ici.

Notu. - Les caractères anatomiques et histologiques ci-dessus mentionnés se rapportent à des plantes recueillies en M.-et-Moselle, et présentant les caractères typiques de l'A. maculatum.

\section{II. - ARUM ITALICUM. MILLER.}

Sin. - A. albispalhum IIont. Plurim. nec Steven.

- numidicum Schотт.

- maculatum ALL. nec L., UGria, SAVI.

\section{$1^{\circ}$ Caractères organographiques.}

La similitude de bien des caractères appartenant aux Arum maculatum et italicum nous dispensera de nombreuses redites, dans la description de ce dernier. Nous nous bornerons à indiquer les caractères différentiels les plus saillants, qu'il présente avec l'A. macutatum. Le pétiole, à gaine large et courte, est plus long du double, ou plus, que le limbe; celui-ci, largement hasté, ou étroitement hastésagitté, à lobe supérieur deux fois plus long que les inférieurs, ces derniers divariqués, séparés par un sinus profond, étroit ou mème large; il est parcouru par trois nervures principales, épaisses, plus pâles que le parenchyme.

Le pédoncule du spadice, moitié plus long que le pétiole, porte une spathe, at tube oblong-cylindrique, trois ou quatre fois plus court que le limbe, blanc à la face interne, à bord d'un pourpre sale; le linube de la spathe est oblong, acuminé, à face externe verte, à face interne jaune pâle, d'un brun ferrugineux, très pâle à la loase. 
L'inflorescence femelle, cylindrique, trois ou quatre fois plus Iongue que l'infloreseence mîle, courte, est formie de $2-3$ rangres circulatres d'ovaires ovö̈des, d’un rert tris paile, renfles a la base, les fleurs femelles rudimentaires sont disposées sur un pelit nombre de cercles; ees fleurs it base lisse, quelque peu allenure, it sommet élargi, brusquement tronqué en plateau, sont d'un jaune pâle, hérissées de verrues bi- ou trifides, réparties uniformément, ou seulement ì la base; du sommet se délache un style, assez long, allénue, recourbé en croc vers le bas. Les fleurs rudimentairessupérieures sont disposées sur plusieurs cereles. La massue épaisse, digitiforme, jaune, est d'une longueur égale, ou plus rarement double, de celle du manche plus grêle:

Chez $A$. itaticum, la grandeur de la spathe, la longueur de la massue du spadice, et la forme du limbe foliaire sont assez variables.

Les dimensions moyennes de la plante sont :
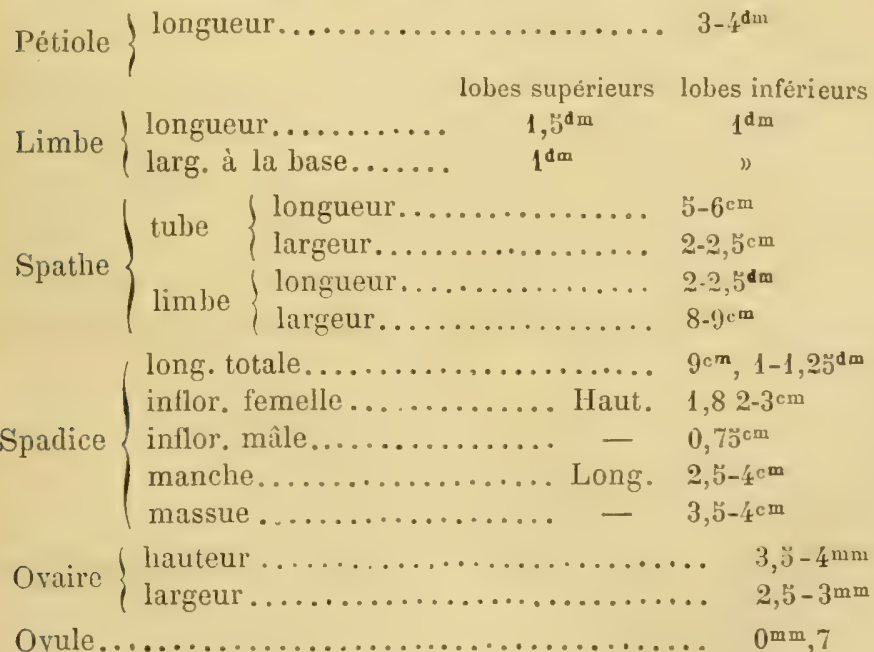

Fleurs stériles inférieures $\left\{\right.$ haut. totale........... $5^{\mathrm{mm}, 0}$

Fleurs steriles inférieures $\left\{\right.$ largeur............. $2^{\text {m }}, 0$

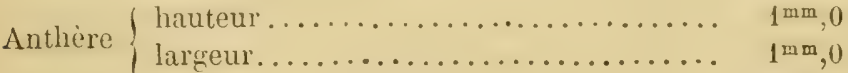

Fleurs stériles supérieures $\left\{\begin{array}{l}\text { hauteur totale......... } \\ \text { largeur. } 6^{\mathrm{mm}}, 0\end{array}\right.$ 


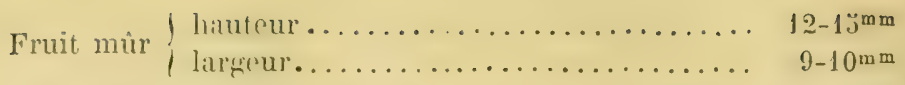

Les différentes variélés et formes d'A. italieum, décrites par les auteurs, ne se rencontrent pas 'n France; elles ne nous intéressent que trop indirectement pour nous arrêter à leur description.

Distribution GÉgraphiQue, - Quelques données sur la répartition géographique de l'A. italicum, dans notre pays, ne seront pas inutiles.

Il existe seul dans la Dordogne, la Gironde, le Lot-et-Garonne, le Tarn-et-Garonne, le Gers, à Narbonne, à Aix, Menton et Monaco. On le trouve à Marscille, à Lyon, il domine à Montpellier, est fréquent dans les plaines de la Haute-Garonne, du Tarn et du Gard, en Auvergne dans les plaines à faible altitude. Il se rencontre, avec une abondance variable selon les localités, dans les Pyrénées. En Normandie, dans la région parisienne, il est fort rare, mais domine de beaucoup dans le Centre et surtout dans l'Ouest; il fait défaut dans les autres régions.

\section{$2^{\circ}$ Caractères anatomiques (1).}

Tubercule. (Portion âgée): - Épiderme exfolié par une assise subéreuse, différenciée à une faible profondeur dans le parenchyme périphérique, formé d'éléments aplatis, faiblement sinueux, alignés assez régulièrement en files radiales (une douzaine d'assises concentriques environ), à parois minces, peu colorables par le vert d'iode, imprégnés par endroits d'une substance colorée en jaune brun, résistant ì la décoloration, même par les hypochlorites (phlobaphène?)

Des plaques de parenchyme mort, à éléments vides, adhèrent, par endroits, à la face externe de l'assise subéreuse.

Parenchyme homogène, au centre comme à la périphérie, ¿̀ éléments obovales, assez réguliers, à parois minces, sćparées par des méats nombreux, de dimensions inégales.

Sur un tubercule examiné à la période de repos, la plupart des cellules parenchymateuses sont :

(1) La structure de l'A. italicum est analogue, et même identique sur bien des points, à celle de l'A. maculatum. Nous ne pouvons done l'étudier en détail, sans nous exposer à des redites. Il y a cependantintérêt, semble-t-il, au point de vue de la clarté de la description, à procéder ainsi. Le médecin pouvant avoir intérêt à distinguer les 2 types, il lui sera plus facilede le faire avec deux descriptions séparées. 

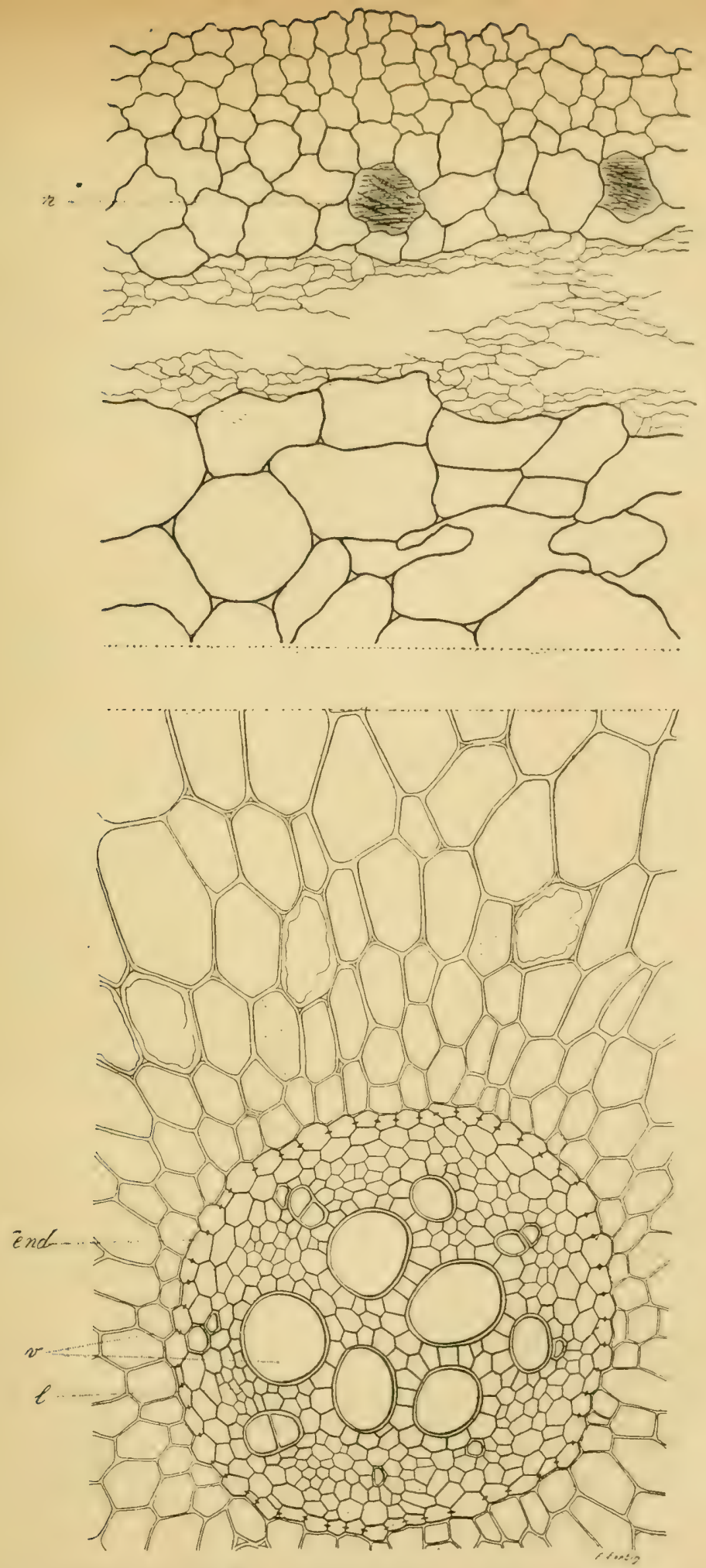

Fig. 19. Arum italicum Mirt. (Gross. 120/1), - Radicelie (struct. prim.). 
$1^{\circ}$ Remplies d'un contenu mucilagineux (coagulable par l'alcool), apparaissant sous la forme d'une masse, rétractée vers le centre de la cellule, sur les coupes traitées par ce réactif, colorables en violet pâle par le carmin aluné.

$2^{\circ}$ Gorgées de grains d'amidon, petits, irréguliers, accolés le plus souvent trois ensemble, la face libre externe convexe, les faces en regard polyédriques par pression réciproque, à hile central punctiforme, peu net, sans fentes radiales.

Faisceaux fibro-vasculaires, nombreux dans toute l'épaisseur de l'organe, ramifiés dans toutes les directions, à vaisseaux uniquement spiralés, faiblement lignifiés, à section polygonale.

Radicelle adventive (structure primaire) (c. au-dessus de la région pilifère). Portion externe de l'écorce nettement différenciée en trois zones, de peu de largeur relativement à l'interne.

$1^{\circ}$ L'externe, formée de deux à trois assises de cellules mortes, polygonales, régulières, sans méals l'assise la plus externe désagrégée par endroits), à éléments plus ou moins saillants vers l'extérieur, fortenent colorables, et bien individualisée seulement sur les coupes colorées.

$2^{\circ}$ La moyenne, peu colorable, à éléments réguliers, polygonaux par pression réciproque, avec rares méats, à parois minces; les raphides de la radicelle sont localisées dans cette zone.

$3^{\circ}$ L'interne à éléments morts, aplatis, onduleux, irréguliers, tassés, la plupart rompus, à parois très minces, peu colorables; de larges lacunes irrégulières, ovalaires, à grand axe tangentiel, résultent de l'écartement et des déchirures, subis par les éléments qui les bordent.

Portion interne de l'écorce uniformément formée d'éléments grands, de dimensions régulièrement décroissantes vers le centre, assez réguliers, la plupart allongés et alignés dans le sens radial, séparés par quelques petits méats, à parois peu épaisses, colorables, munis de fines ponctuations. En quelques rares points, lors de l'accroissement de cette zone (disposition non indiquée par la figure), certaines cellules se sont détachées de leurs voisines (écra- 
sces par la pression exercée par celles-ci), Jeur memlirane, restie très mince, se trouve plissée et plus ou moins replices sur ellemême.

Endoderme (end.) très net, avec cadres d'épaississement bien visibles sur le milieu des parois radiales.

Rien ne distingrue le péricycle du reste du parenchyme homogène, à éléments réguliers, polygonaux, sans méats, dont la paroi mince se colore diflicilement, qui constitue la masse du rylindre central.

Ilots libériens (l) à peine distincts du parenchyme ambiant, coins ligneux, à pointe externe, les petits vaisseaux plus ou moins elliptiques ou polygonaux, les vaisseaux plus jeunes, larges, ì section circulaire, tous peu lignifiés $(v)$.

Feuille $n^{0} 1$ Pétiole.

C. initiule (à mi-hauteur de la portion vaginale du pétiole, en fer

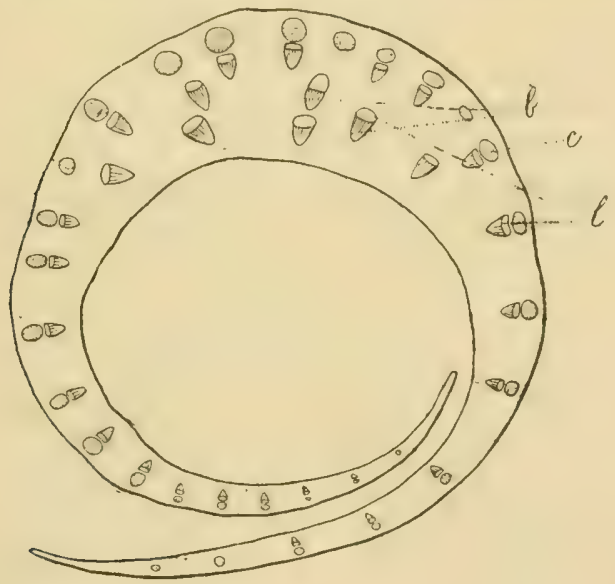

Fig. 20. Arum italicum MiLl. (Gross. 7/1). - Pétiole (c. initiale). (semi-schém.)

à cheval, à branches aiguës, l'une chevauchant sur l'autre; faisceaux tous à bois interne, sur deux lignes concentriques dans la région médiane renflée śs une seule ligne dans la portion amincie qui constitue la gaine;, la ligne interne dédoublée en partie.

C. médiane (à la pointe de la gaine) subcirculaire, atténuée en 
pointe sur la face interne, faisceaux sur deux lignes concentriques, l'externe de 13 faisceaux, périphérique, très régulière, en arc à petite ouverture interne, l'interne en arc irrégulier, de 6 faisceaux,

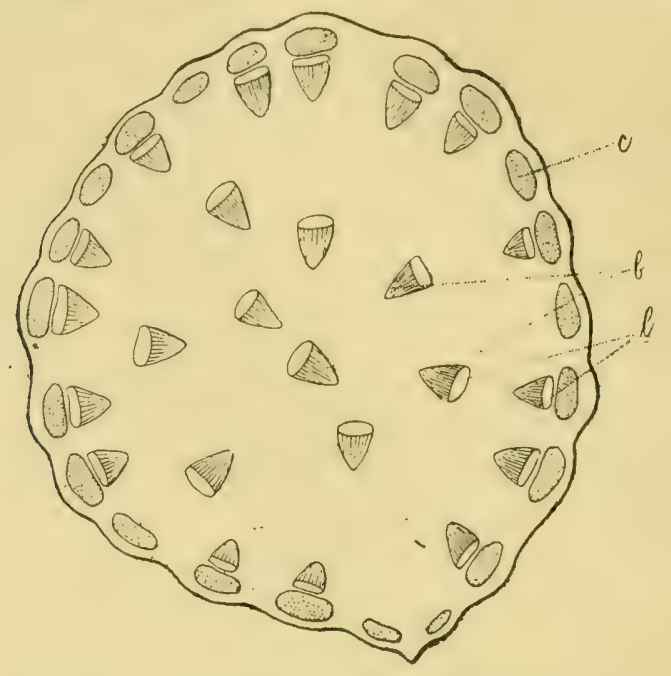

Fig. 21. Arum italicum Mrte. (Gross. 10/1). - Pétiole (C. médiane). (semi-schém.)

ouvert du côté interne, dans cet arc 3 faisceaux centraux, à bois interne.

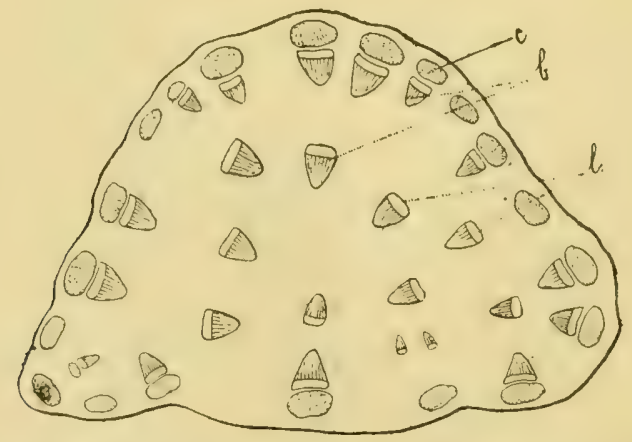

Fig. 22. Arum italicum Mur. (Gross. 10/1). - Pétiole (C. terminale). (semi-schém.)

C. terminale (ou caractéristique) (à la naissance du limbe), triangulaire, curviligne, à base interne, deux ailes s'ébauchent aux angles latéro-internes (naissance du limbe). Les 15 faisceaux de 
l'arc externe s'y retrouvent, un dédoublé, un autre à situation médiane inverse, 1 des faisceaux centraux a disparu, les 2 autres ont accompli une rotation, jusqu'ì placer leur bois vers le côté externe.

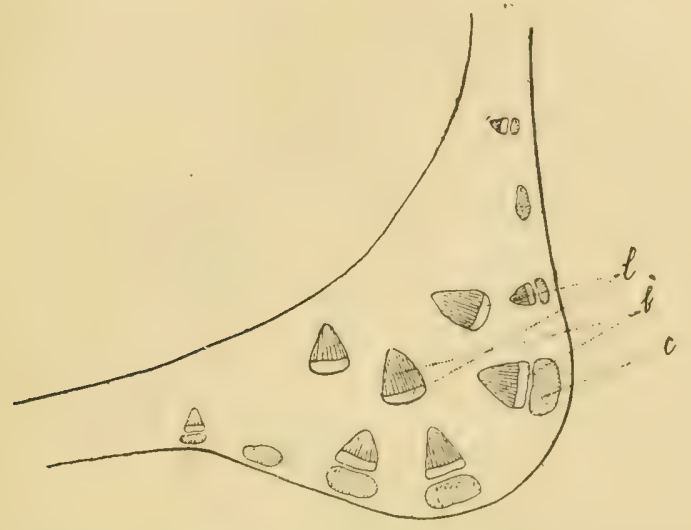

Fig. 23. Arum italicum Mrul. (Gross. 9/1). - Nervure médiane du limbe (C. médiane) - (semi-schém.)

Limbe. C. Au milieu de la nerv. médiane. Deux arcs concentriques, très ouverts, de faisceaux; 6 faisceaux seulement persistent à la périphérie, et 3 de l'arc interne, les autres se sont épuisés pour fournir des nervures au limbe.

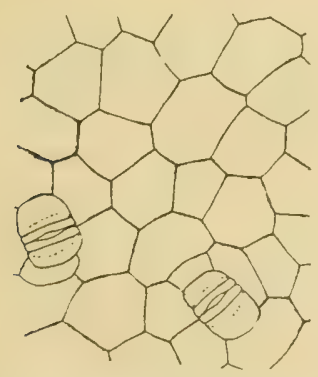

Fig. 24. Arum italicum Millu. (Gross. 120/1). - Epiderme supérieur.

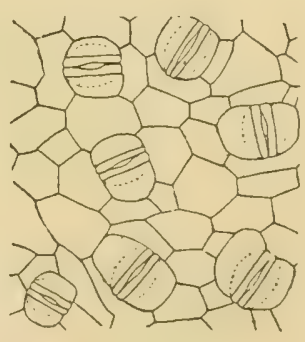

Fig. 25. Arum italicum MiLL. (Gross.120/1). - Epiderme inférieur. Limbe.

La coupe transversale à ces divers niveaux est toujours légèrement onduleuse, chaque ondulation correspond à la saillie d'un 
massif de collenchyme, la plupart de ces massifs sont adossés à un faisceau.

A tous les niveaux, on constate :

Epiderme à éléments polygonaux, allongés dans les portions superposées aux saillies des cannelures, à éléments quadrangu-

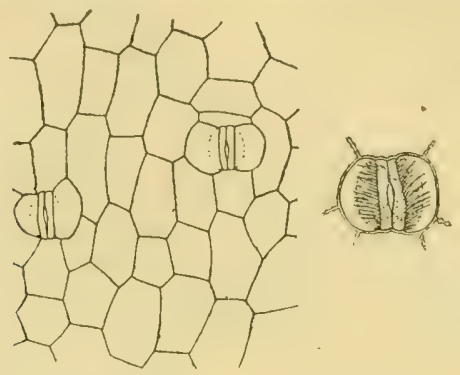

Fig. 26. Arum ilalicum Mru. (Gross. 120/1). - Epiderme du pètiole.

Stomate isolé, plus grossi.

laires (sur la c. transvers.), un peu plus petits et aplatis en face des massifs de collenchyme, à parois minces (l'externe un peu plus épaisse), à culicule striée; exoderme externe à éléments plus petits que ceux du parenchyme sous-jacent; les raphides sont, assez exactement, localisés dans celte assise ou les assises sousjacentes.

Aucun caractère net ne distingue le parenchyme cortical du parenchyme central, éléments plus petits dans le premier, à parois minces, nombreux méats, lacunes nombreuses, larges surtout vers le centre, assez régulières, bordées d'éléments, tassés, déchirés, allongés longitudinalement en formes de poches.

Massifs de collenchyne à parois régulières, dont l'épaisseur peu notable n'est que peu augmentée aux angles, sur les coupes naturelles; l'épaisseur des parois augmente notablement sous l'influence des réactifs hydratants, et la lumière se trouve réduite à une fente étoilće. Faisceaux fibro-vasculaires, privés de tout élément de soutien, vaisseaux uniquement spiralés.

Le parenchyme assimilateur vert est réduit à une mince couche périphérique, et ne s'étend qu'à une faible profondeur, suivant les 
travées qui séparent les lacunes. Ditns ses élements, surtout it la face supérieure de la nervure médiane, on trouve les masses gommeuses dont nous parlerons à propos du limbe.

Aux niveaux supérieurs, dans les intervalles entre les massifs collenchymateux, il y a une tendance plus ou moins marquée au décollement de l'exoderme, qui se délache de la face interne de l'épiderme, et limite avec elle des fentes étroites.

Stomates répartis surtout a la face interne, dans la concavité supéricure de la nervure médiane, semblables à ceux du limbe, chacun d'eux superposé à une chambre sous-slomatique, placés sur un plan légèrement inférieur, par rapport à celui de l'épiderme.

A la face externe, stomates bien moins nombreux, localisés aux dépressions limitées par les cannelures, et par suite presque tous superposés aux fentes de décollement existant dans ces dépressions.

Limbe Epid. à éléments polygonaux, ou faiblement onduleux, allongés au-dessus du trajet des nervures, cuticule faiblement

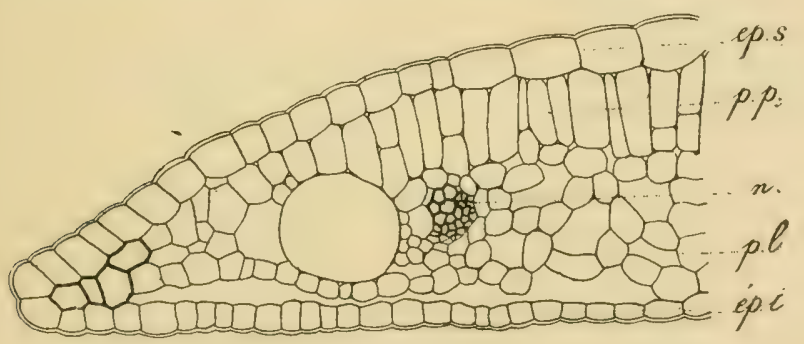

Fig. 27. Anum italicum MItL. (Gross. 120/1). - Limbe (c, transversale). Portion marginale.

striée, à parois minces, plus petits à la face inférieure (sur c. transvers.). Stomates présents sur les deux faces, maissurlout nombreux à la face inférieure, obovales dans l'ensemble, accompagnés de deux, parfois trois ou quatre cellules annexes (par cloisonnement radial de l'une ou de deux cellules annexes primitives), latérales, semi-lunaires, convexo-concaves; la face externe des cellules stomatiques, surplombe, en se rabattant en dedans, l'ostiole; leurs 
deux lèvres externes, nettement et finement striées, limitent ainsi une petite chambre sus-otiolaire.

Mésophylle unifacial: deux assises de parenchyme palissadique à la face supérieure, assise supérieure d'éléments allongés, assise inférieure disparaissant par endroits, perdant de sa netteté au bord extrême du limbe (fig. 2i ) et formée là d'éléments plus courts, plus larges, quelque peu irréguliers.

Mésophylle inférieur, lacuneux, à èléments irréguliers, limitant de petites chambres sous-stomatiques ; quelques cellules, obsphéniques, alignées de distance en distance, dans la partie inférieure du mésophylle, contenant des raphides.

Au bord extrème du limbe, quelques éléments du mésophylle épaississent leur paroi, pour servir d'éléments de soutien.

Nervures isolées à mi-hauteur du mésophylle, entourées d'une gaine de parenchyme condensé, dépourvues de tout élément de soutien différencié, et n'ayant que quelques éléments vasculaires spiralés.

Mème contenu gommeux, dans la plupart des cellules, que dans l'A. maculatum.

Pédoncule duspadice. - Présente à peu près les caractères de celui de l'A. maculatum.

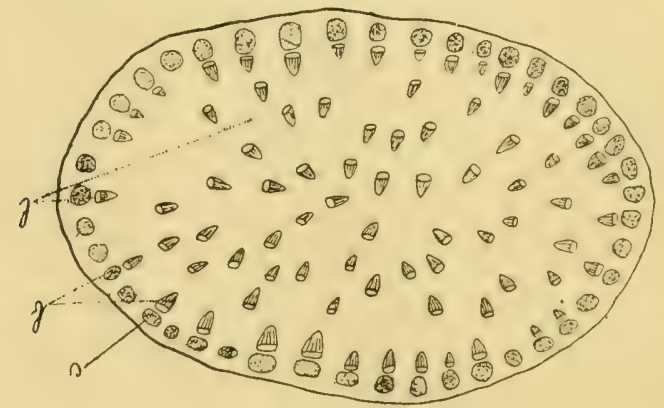

Fig. 25. Arum italicum MrLl. (Gross. 120/1). - Pédoncule du spadice. (c. à mi-hauteur). (semi-schém.)

Un cercle périphérique, très régulier, contient (pratiqué à mihauteur de l'organe) 33 faisceaux; les faisceaux internes se répartissent assez bien sur 3 cercles assez irréguliers, de sorte que l'en- 
semble des faisceaux est ì peu près orienté symétriquement, par rapport à l'axe de l'organe.

Spathe. - Épidermes: mèmes caractères que dans l'A. murulutum. Hypoderme externe muni, de distance en distance, de gros éléments raphidifères.

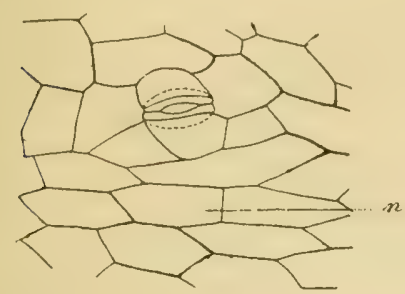

Fig. 29. Arum ilalicum Mill. (Gross. 120/1). - Epiderme externe. sl stomate.

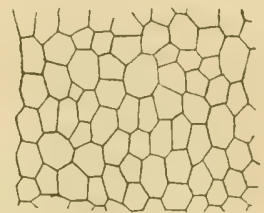

Fig. 30. Arum ilalicum MrLL. (Gross. 120/1). - Epiderme interne.

Spathe.

Mésophylle à éléments assez réguliers, obquadrangulaires, liche au centre, condensé à la périphérie, limitant de nombreuses lacunes aérifères, centrales, alignées sur un cercle unique, à égalle distance desdeux faces. Nervures principales, isolées au sein du mésophylle; au dos des plus grosses, un faisceau de collenchyme tangent à l'épiderme externe, un cercle de petites nervures accessoires, immédiatement siluées au-dessous de l'épiderme interne; chacune de ses petites nervures provoque une saillie de l'hypoderme et de l'épiderme, à la face interne.

Hypoderme interne, à éléments quelque peu allongés, alignés en assise subpalissadique, sauf au-dessus des petites nervures, ou il se dédouble en deux assises de petites cellules polygonales.

Fruit. - Le péricarpe mûr comprend :

$1^{\circ}$ Un épiderme externe (épicarpe) à cellules tabulaires (en coupe) à paroi externe épaissie, plus ou moins polygonales (vues de face) ou rectangulaires, avec quelques stomates, placés sur un plan inférieur à celui de l'épiderme, construits sur le type des stomates des organes végétatifs.

$2^{\circ}$ Une chair (mésocarpe) à grands élénents sphéroïdaux, entremêlés de poches à raphides. 
$3^{\circ}$ Un épiderne interne (endocarpe) à cellules tabulaires (en coupe) à paroi interne bien épaissie, polygorales (rues de face), allongées

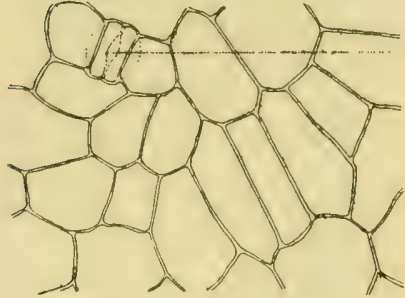

Fig. 31. Arum italicum MiLl. (Gross. 120/1). - Epicarpe. s, stomate.

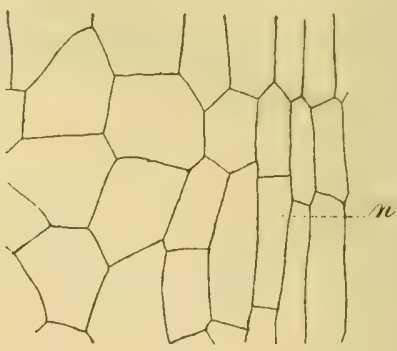

Fig. 32. Arum italicum MrLL. . (Gross. 120/1). - Endocarpe. n, portion superposée à une nervure.

sur le trajet des nervures qui parcourent le mésocarpe; totalement dépourvu de stomates.

Le prigment ronge des fruits d'Arum se présente en granulations amorphes, engagées dans des leucites d'un brun rouge brique, arrondis.

La solution éthérée de ce pigment est jaune orange, et ne dépose que difficilement des cristaux. Ce fait serait dû à ce que, dans ces fruits, le pigment cristallisable se trouve associé à un pigment jaune amorphe, présentant les mêmes caractères que les chromoleucites jaunes des fleurs, pigment soluble aussi dansl'éther.

Les cristaux quel'on peutobtenir, parévaporation de la solution éthérée, sont:

$1^{\circ}$ Des lamelles à bords irréguliers, ou bien de longues et étroites lamelles, terminées en pointe, d'un rouge orangé.

$2^{\circ}$ Des tables rhomboïdales, d'un rouge carmin, lorsqu'elles atteignent une certaine épaisseur, d'un jaune orangé, lorsqu'elles sont en lames minces. Nous reviendrons au chap. VI sur l'intérèt que peut présenter ce pigment, pour „une détermination médico-légale des fruits d'Arum.

Graine. - A maturité, les graines d'Arum présentent à l'œil nu, en 
apparence, deux téguments, dont l'interne apparait sous la forme d'une zono: plus foncée (fig. 34, 34 lis). Au microscope, celte zone se

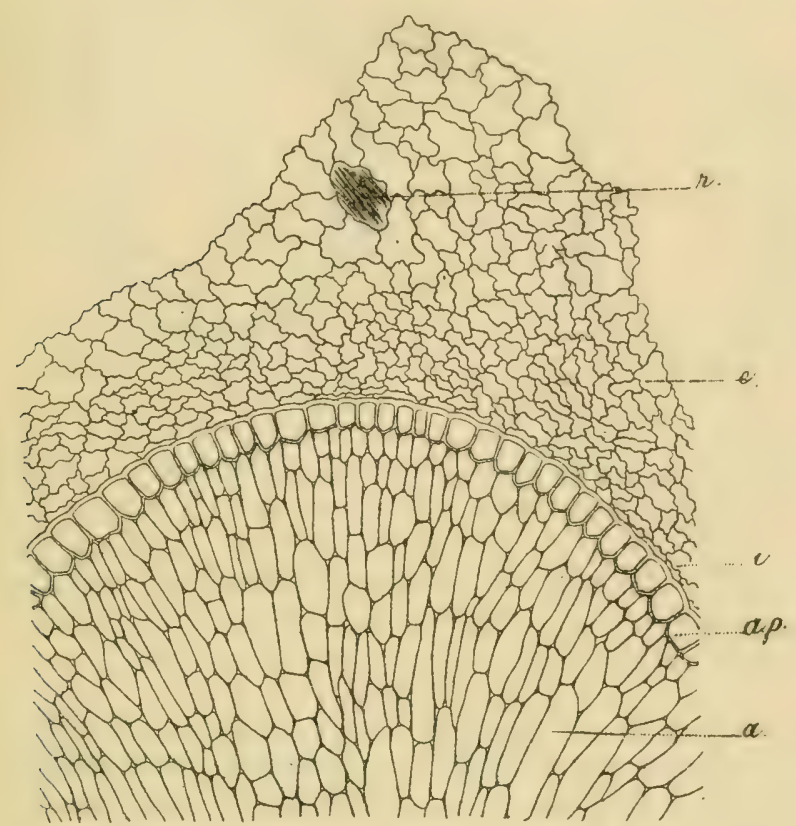

Fig. 33. Arum italicum Mils. (Gross. 40/1). - Graino (c. transvers.). $e$ c. externe du tég., $i$ c. int. du tég., $r$ cell. à raphides, $a$ albumen, ap assise périphérique de l'albumen.

décompose en petits éléments pressés, qui se confondent insensiblement avec ceux de la zone périphérique; à cet àge, il est difficile de distinguer anatomiquement deux téguments. Nous n'arons pas étudié l'histogenèse de celle graine; mais, d’après Braxdza, elle ne présenterait, à aucun àge, deux téguments (ce qui paraît contraire à la manière d'être de la plupart des Aroüdées). Ce point spécial ne présente du reste qu'un intérêt purement botanique.

Cà et là, dans l'épaisseur du tégument, on trouve des éléments raphidifères.

La coupe longitudinale (fig. 34 bis) montre, mieux que ne le ferait toute description, les particularités de stıucture de cette graine.

L’albumen est limité par une couche périphérique de grosses 
cellules cubiques, à parois épaissies, et sa masse formée d'éléments

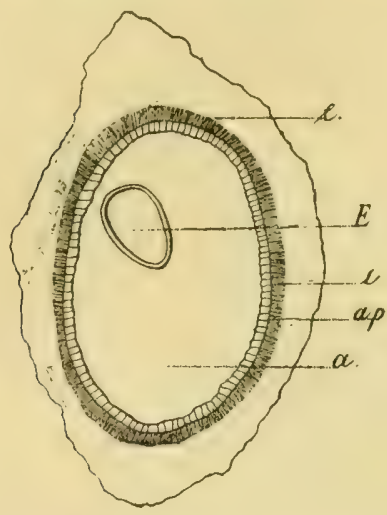

Fig. 3'. Arum ilalicum Mirl. (Gross. 8/1). - (sémi-schẻm.).

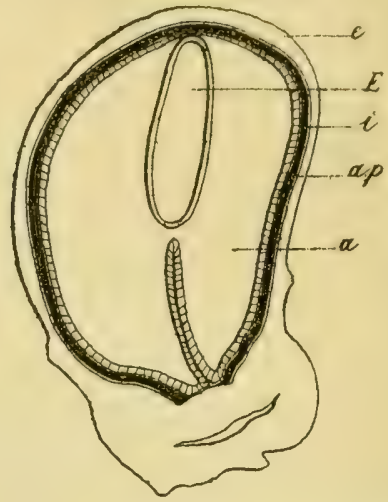

Fig. 34 bis. Arum italicum MiLL. (Gross. 8/1.) - (sémi-schém.).

à parois minces, la plupart allongés et alignés en files radiales, à contenu amylacé.

Nota. - Les caractères anatomiques et histologiques, ci-dessus mentionnés, se rapportent à des pieds d'A. italicum cultivés au Jardin de la Faculté à Paris, et appartenant à une variété à nervures presque blanches (sauf en ce qui concerne les fruits et les graines, étudiés sur des échantillons recueillis dans la Loire-Inférieure.)

Il serait superflu de discuter ici la valeur spécifique de l'A. italicum. Regardé par les uns comme espèce indépendante, il est assimilé par les autres à une simple variété de l'A. maculatum. La grande similitude des caractères, tantorganographiquesqu'anatomiques, pourrait plaider plutòt en faveur de cette dernière opinion. Mais ce point spécial offre trop peu d'intérêt médical, pour nous arrêter au cours de cette étude déjà trop longue.

Les deux espèces : maculatum et italicum, sont classées toutes deux, avec les formes qui s'y rattachent dans une sous-section du genre Arum, celle des Ootuberosa, caractérisée par un tubercule ovoïde ou oblong, placé horizontalement dans le sol, et du sommet duquel émergent les pousses feuillées et les inflorescences. 
Chaque espèce est cependant placée dans un petil groupe speicial, distinct du voisin, par quelques caractères.

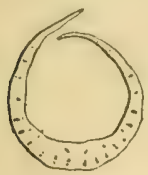

1

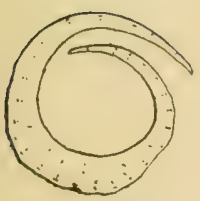

1

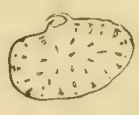

2

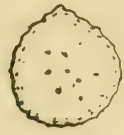

2

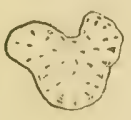

3

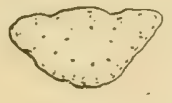

3

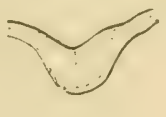

4

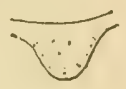

\&

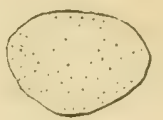

5

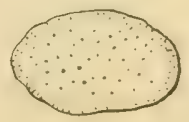

5

Fig. 35. Comparaison entre les coupes du pétiole et de l'axe d'inflorescence des I Arum maculalum L. II Arum italicum MıLL. 1, 2, 3, Pétiole. c. initiale, médiane, terminale. 4 nerv. méd., 5 axe du spadice. (semi-schéma).

(Gross. 2/1).

De ces 2 groupes:

Le $1^{\mathrm{er}} \mathrm{A}$. (Arum maculatum) a la massue du spadice subconoïde ou cylindroïde, portée par un pied grêle, allongé, qui s'épaissit brusquement pour la former, violette.

Le $2^{\circ}$ B. (Arum italicum) à la massue du spadice digitiforme ou conique-digitiforme, jaune ou jaune pâle, parfois violette.

Bibl. 15ว2. - Tragus. Stirpium libri tres, (p. 773-4).

1588. - Tabernemontanus (J. K.) Neuw. Kreuterbuch.

1588. - Camerarius (Joachim.). Hortus medicus (p. 22).

1588. - Fuchs (L.). Hist. plant., (53, ch. XXII. Lyon, G. Rouille, in-4 $4^{\circ}$, trad. franc, du Neuw Kreuterbuch).

1598. - Matthiolus (P.) Commentarii Dioscoridis (tr. fr. de Pinet (A.).

1651. Bauhin (J.). Hist. plant. univ., II (p. 784.)

1701. - Wedelius (G.-TV.). De Aro (Resp. Schalhass, Ienæ, in- $4^{0}$.)

1701. - Wedelius (G.-W.) (Pr.), De pone Dyrrachino Julii Cæsaris. (Cum vita candidati Joh. Cruesti Ronsche. bach). Sm. in-4․ Ienæ.)

1793. - Linné (C.). Species plant. (1'e édit. et $2^{\mathrm{e}}$ édit., p. 1370).

1765. - Gouan. Flora Mlonspeliaca (p. 405).

1769. - Oeder (G.-C.), Flor. Dan. (t. 505). 
1778-1794. - LAMARCK. Flor. frane. (1 ${ }^{\text {re }}$ et $2^{\mathrm{e}}$ éd., III, p. 337-538). 178ä-90. - Miller (P.-L.). Dict. des jardiniers.

178ว. - Allioni (C.). Flora Pedemontana (II, 228).

1789. - Lamarci (J.-B.). Dict. bot. de l'Encycl. (III, p. 8-9).

1789. - Ucria (B.-L.). Hortus regius Panhormitanus (p. 389).

1791. - WitmMann. Summa plant.

1798. - Savi (P.). Flora Pisana (II, 370).

1803. - Dubois (F.-N.-A.). Méth, éprouv.

1804. - Desfontaines (R.-L.). Tableau de l'Ec. de Botanique du Mus. d hist. nat.

1804. - Lestidooubois (Th.). Botanographie universelle. (II, p. 3วั5).

1805. - De Candolle (A.-P.) et Lamanck (J.-B.) Flore franc.. (III, p. 152).

1805. - Jaume Saint-Hilaire (J.-H.). Expos, Fam.nat.

1813. - Targioni Tozzetti (O.). Istit. bot. (III, 378).

18:8. - Loiseleur-DeslongChayps (J.-L.-A.). Flora gallica. 1833-60. - NeEs von Esenbeck (Tr.). Genera plant.Fl.german. $(2$, t. 5).

1835. - Blume (K.). Rumphia. (I, p. 117, 118).

1838. - Brandt (Рн.) und Ratzebung (J. T.). Giftgev. (р. 33, t. 7$)$.

1841. - KunTH (K. S.). Enumer. plant. (III, 23-24).

1850. - Irinsch (Thilo.) Zur Morphol. der monokotyl. Knollen und Zwiebelgewächse (in-8* Berlin. G. Reimer, p. 164166, tab. X, fig. 34-39)

18:2. - Parlatone (F.). Flora italiana (II, p. 244).

18ӟ4-ə̆з̆. - Sснотт (II. W.). Araceen Betreffendes (Wien. 8º). $10)$.

Synopsis Aroidearum (Wien, $8^{\circ}$,

1856. - Inmisch (Th.). Aroideen (Berlin, 4).

1858. - Sснотт (H.- W.). Genera Aroidearum Vindobonæ fol. 1860. - $) \quad P r o d r$. systematis Aroidearum (Vindobonæ, 8, 82, 83).

1861. - Polonio. Osservazioni organogeniche sui foretti feminei dell Arum italicum.

1866. - Van Tieghem(P.). Rech. sur la struct. des Aroïdées. (Ann. se. natur., Bot., sér. 5, VI, p. 72-210).

1879. - Engler (Araceæ in De Candolle. Monog. Phanerog.).

1886. - Dalitzsch (MAX). Beitr. z. Kenntniss der Blattanatomie der Aroideen. (Botan. Centralblatt, Bd. XXV. Taf. III).

1888. - Counchet. Rech. sur les Chromoleucites. (Thèses Fac. des Sc. Paris, série A. no 111, no d'ordre 637, p. 26.95, pl. XVIII, fig. 4.) 
1889. - Berthenand (E.-L.). L'Anum italicua au point de vue botanique, bromalologique et médical, in-8 ${ }^{\circ}$, Alger.

1890. - Gancin. (A. G.). Rech. sur l'histogenèse des péricarpes charnus. (T'hèse Fac. des sc. Paris, sér. A. no 14́, $\mathrm{n}^{\circ}$ d'ordre 710, p. 394-6).

1891. - Brandza (M.). Développement des téguments de la graine. (Thèses F'ac. des Sc. Paris, série A. $\mathrm{n}^{\circ} 148$, $\mathrm{n}^{\circ}$ d'ordre 706. (Paris, P. Klincksieck, Aroidées, p. 24).

1885. - Chos (D.). Les Arum vulgare Lam k, et italicum Mill. (Bull. de la Soc. Bot. de France, t. XLII, p. 460464.)

1896. - Arcangeli (G.). Sull'A rum italicum Mill. (Bull. della Soc. bot, ital., nº 9, p. 321-24).

1897. - Argangeli. Ancora sull'Arum italicum. (ibid., $\mathrm{n}^{\circ} 1$ p. 46.8).

- Derpino. Att. del. Soc. Ital. d. Sc. Nat,, vol. XI, p. 282.

Pour la répartition géographique des Arum.

Bibl. - Bautier (A.). Flores part. de la France (p. 269). 1868.

Bautier (A.). Tabl. analyt. dela floreparisienne $\left(21^{\circ}\right.$ édit. 1887. p. 300).

BONNET. Petite flore paris. (p. 406).

Boreau. Flore du Centre ( $3^{e}$ éd.).

Cosson et Germain de Saint-Pienre, Flore des environs de Paris ( $3^{\mathrm{e}}$ éd., p. 493.1876).

Delastre. Flore de la Vienne, 1842.

Dulac. Flor. des Hautes-Pyrén. (p. 46-7).

Guillaud. Flore de Bordeaux (p. 173). 1883.

Kirschleger. Flore d'Alsace (II, 207.) 1852-58.

LECOQ. Géogr. bot. de l'Europe (III, p. 475-76). 1858.

Lloyd et Foucaud, - Flore de l'ouest.

LoRey et Duret. Flore de la Côte-d'Or.

La Peynouse ( $\mathrm{PH}_{\mathrm{H}}$. PICOT De) Hist. abreg. des $\mathrm{pl}$. des Pyrénées. (Supplém. 8, XII, p. 143). 1818.

Royer $\left(\mathrm{CH}_{\circ}\right)$. Flore de la Côte-d'Or; p. (528-9).

Serres. Flore de Toulouse (p. 22). 1836.

Thuillier. Flor. des envir. de Paris.

Tournon (D. J.). Flore de Toulıuse, 1811.

Zetterstedt. Plant. vasc. Pyrén. (p. 263). 1857. 


\section{Explication des figures de la planche $I$.}

\section{Arum italicum MıLL.}

Fig. 1. - Port de la plante, un peu avant la floraison (axe du spadice sectionne à mi-hauteur).

2. - Jeune feuille, lors de son épanouissement, pour montrer la préfoliation........................... $\frac{1}{2}$

3. - Fragment du limbe foliaire, pour montrer la nervation....... $\frac{1}{1}$

4. - Spadice avant l'anthère............................ $\frac{1}{2,5}$

5. - Spadice épanoui........................... $\frac{1}{5}$

6. - Fragment de la spathe, pour montrer la nervation.......... 1

7. - Spadice, la spathe détachée...................... 1

8. - Un ovaire (face externe).........................

9. - Un ovaire (coupe longitudinale)................... $\frac{6}{1}$

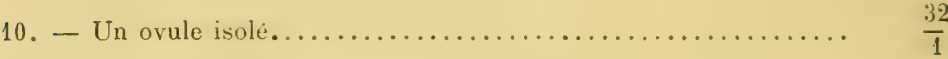

11. - Un ovule (coupe longitudinale) $\ldots \ldots \ldots \ldots \ldots \ldots \ldots \ldots \ldots \ldots \ldots \ldots \ldots \ldots \ldots$

12-13, - Fleur neutre inférieure, gross. $\frac{10}{1}$, et appendices qui la sur-

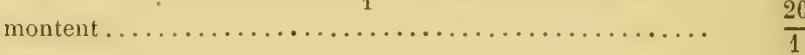

14-15. - Étamine (facs supérieure et face inférieure).......... $\frac{14}{1}$

16. - Fleur neutre supérieure...................... $\frac{10}{1}$

17. - Spadice fructifié................................. $\frac{1}{2,5}$

18. - Fruit isolè (face latérale) ....................... $\frac{2}{1}$

19. - Fruit isolé (coupe longitudinale).................. $\frac{2}{1}$

20-21. - Graine isolée et coupe longitudinale................ $\frac{3}{1}$

22. - Embryon isolé................................ $\frac{5}{1}$

23-24-25-26. - Germination et développement de la plantule...... $\frac{1}{1}$ 



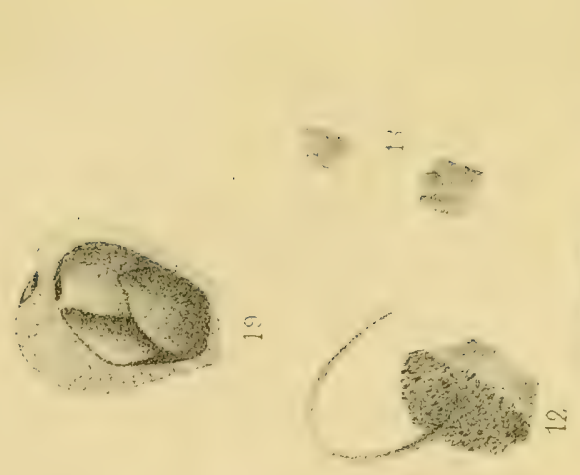

-
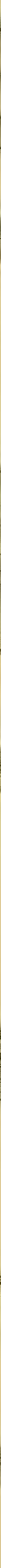



\section{B. - Arisarum vulgare TArg. T'ozz.}

Syn. - Arisarum australe Rrch.

Arum Arisarum L.

- incurvatum LAIr.

\section{$1^{\circ}$ Mode de végétation (1).}

L'A. vulgare est une herbe, dont le mode de végétation rappelle beaucoup celui des Arum.

Nous n'avons pu suivre les premières phases du développement, et les auteurs sont muets sur tout ce qui concerne le mode de végétation et de ramificalion de la plante.

Un point qui présente, pour le médecin, un intérêt spécial est la forme très variable de la portion souterraine, parfois purement rhizomateuse ou tuberculeuse, le plus souvent mixte.

Lorsqu'il n'y a qu'un tubercule ovoïde, à direction horizontale, ou plus ou moins verticale, la plante rappelle de tous points un Arum. Mème portion inférieure, àgée, flétrie (visible d'ailleurs sur tous les pieds rhizomateux outuberculeux), et prête à se séparer de la portion plus jeune du tubercule (fig. 7, pl. III); même cercle de radicelles adrentires, à la base de la pousse de l'année; mèmes cicatrices peu nettes, à la surface du tubercule, des écailles et des feuilles de l'année précédente, avec des bourgeons axillaires dormants (plus ou moins déreloppés), apparaissant sous forme de petites proéminences nacrées; mèmes feuilles (2 ou 3), réduites à leur portion vaginale écailleuse, à la base de la pousse verte.

Cette pousse épanouit, sur les pieds prèts à fleurir, 2-3 rarement plus) feuilles vertes complètes. A l'aisselle de la première des feuilles vertes, souvent flétrie de bonne heure (par suite facile ì rabattre), on trouve souvent deux axes d'inflorescence, d'âge différent. Le plus jeune a déjà fructifié, alors que l'autre est encore à peine épanoui;

(1) Les caractères indiqués ci-dessous pour cette plante se rapportent ì des échantillons recueillis aux environs de Nice. 
ce dernier est situé à l'aisselle d'une bractée latérale par rapport au premier axe d'inflorescence (fig. 2, pl. III).

La deuxième feuille verte, sensiblement opposće à la première, porte ì son aisselle un bourgeon de 4-כ̈ feuilles complètes, opposées, dont la première s'épanouit en mème temps que la feuille axillaire du bourgeon, par rapport à laquelle elle est latérale.

La plupart des tubercules portent, à leur partie supérieure et latéralement, un bourgeon à direction sensiblement horizontale, (fig. 4, $\tilde{3}$, pl. III). Ce bourgeon est l'ébauche de la ramification latérale et horizontale de la plante. L'axe de ce bourgeon est formé par une sorte de talon, correspondant à la base du bourgeon axillaire de la deuxième feuille verte (fig. 5,6, pl. III). A mesure qu'il croit, ce talon déchire, pour se frayer un passage, la base vaginale des feuilles successives du bourgeon dont il émane. Ces gaines perforées sont très visibles, tout autour du bourgeon horizontal en voie d'accroissement. Celui-ci émet, à sa partie inférieure, e tà chaque entrenœud, une longue racine adventive, d'abord tubériforme allongée, rapidement ratatinée, puiscaduque. Ces bourgeons peuvent se séparer du tubercule (et mener une vie indépendante), ou se développer en une portion rhizomateuse plus ou moins longue, attenante au tubercule primitif (fig. 1. pl. II). Il est rare que la portion souterraine soit tout entière rhizomateuse (fig. $8, \mathrm{pl}$. III), le plus souvent le rhizome ne tarde pas à se renfler en un tubercule, d'où émergent la pousse de l'année et le bourgeon horizontal destiné à assurer la végétation ultérieure de la plante (fig. 2, pl. II); Parfoisaussi, le bourgeon latéral, issu d'une vieille portion rhizomateuse, épaissit sa base en tubercule, en mème temps qu'il épanouit ses feuilles vertes, et devient ainsi, après son isolement, l'origine d'un tubercule indépendant (fig. 1, pl. III.)

(Ce serait sortir du cadre spécial de ce travail que de discuter ici la nature monopodique ou sympodique du rhizome ou du tubercule).

\section{$2^{\circ}$ Caractères organographiques.}

Le pétiole des feuilles complètes de la plante adulte est long, 
muni à sa base d'une gaine assez courte, parcouru dans sa partie supérieure d'un sillon médian, plus ou moins couvert de macules, oblongutes ou linéaires, éparses ou agminées, d'un noir pourpré.

Le limbe, trois ou quatre fois plus court que le pétiole, est ovale-sagilté, ou triangulaire sagitté, parcouru de chaque cìté de sa nervure médiane, par "a-6 nervures, incurvées, surtout les postérieures, se raccordant en anses sur le bord; une nervure, tout à fait marginale, est formée d'ares superposés aux nervures sousjacentes.

On peut décrire le limbe comme formé de 3 lobes : le supérieur courtement apiculé, les deux postérieurs ovales, oblongs-ovales, ou même subtriangulaires, obtus le plus souvent, nettement inégaux, séparés par un sinus plus ou moins large, dont le fond est arrondi.

Comme chez les Arum, les feuilles émises par les jeunes plantes, pendant leur première année de végétation (fig. 1, pl. III), ne possèdent qu'un limbe entier. Ces jeunes feuilles présentent pour le médecin le même intérèt pratique que les jeunes feuilles d'Arum.

L'axe d'inflorescence atteint, le plussouvent, la longueur du pétiole et est maculé comme lui.

La spathe s'insère obliquement sur le spadice, elle forme un tube subcylindrique, légèrement renflé à la base, puis quelque peu rétréci, ouvert seulement dans le haut et latéralement. Son ouverture, un peu oblique de haut en bas, est obovale dans l'ensemble, acuminée vers le haut, limitée par 2 lèvres (l'une recourrant l'autre avant l'anthère), finalement incurvées en dehors, surtout dans la partie inférieure. La face extérieure de la spathe est d'un vert pâle ou presque blanche, d'un vert pourpré foncé au sommet, parcourue de nervures saillantes, d'un vert foncé; sa face interne est toute lavée de pourpre à sa partie supérieure, et d'un vert jaunâtre pàle, sur la portion incurvée des lèvres, qui limitent l'ouverture. Le spadice a la forme d'un cône très allongé, incurvé au sommet, parallèlement à la courbure de la spathe, terminé par une massue grèle, olivaire, légèrement exserte, qui se relie insen- 
siblement à son manche, blanc à sa base; le spadice est, dans sa parlie recourbée, d'un jaune verdâtre pâle.

L’inflorescence est monoïque.

L'infloresrence femelle, unilatérale, est localisée à la base du spadice, de son côté ventral, réduite à $3-5$ fleurs. Celles-ci, intimement pressées les unes contre les autres, el polyédriques par pression réciproque, sont constituées par un seul ovaire, ayant la forme de 2 troncs de pyramide à $4-\ddot{b}$ pans, accolés, et surbaissés, surtout le supérieur, qui a des arêtes mousses parcourues, ainsi que le milieu des faces, par des lignes longitudinales brunes. Chaque ovaire est surmonté d'un style court, cỵlindrique, terminé par une petite tête sphérique, hérissée de papilles stigmatiques. La loge unique de l'ovaire renferme, insérés sur un placenta basilaire qui occupe toute sa base, un nombre rariable d'orules, bitégumentés, et dont le nucelle présente les particularités signalées à propos des Arum (TrLasve) ; entre les ovules, le placenta émet d'innombrables filaments grêles, sous forme d'un duvet long et soyeux. Les orules, primitivement orthotropes, dressés, à funicule court, viennent, en s'accroissant, à butter contre la paroi supérieure de la loge ovarienne; ils s'incurvent alors, pour trouver à se loger, et présentent une tendance plus ou moins accentuée à l'anatropie. (Il est intéressant de noter que le degré d'anatropie de l'ovule est absolument déterminé par son insertion placentaire; tout orule central s'accroit notablement, vient butter contre la face supérieure, et par suite s'incurve horizontalement; tout ovule périphérique, au contraire, s'accroît peu, il ne s’incurve pour se loger que dans la concavité de la loge ovarienne.)

L'inflorescence mâle, d'une hauteur double de celle de l'inflorescence femelle, est formée de fleurs, éparses au-dessus de fleurs femelles, sur le tiers inférieur du spadice, réduites à une étamine. Celle-ci est formée d'un filet court, rétréci en son milieu, dilaté aux deux extrémités, parsemé de petites macules pourprées, dilaté mais mince, évidé en gouttière à la face dorsale et supérieure de l'anthère et d'une anthère insérée sur le filet, suivant le mode pelté, à connectif également maculé ; celle-ci a des 
loges oblongues, horizontiles, confluentes, sourrant horizonlalement par une fente continue. Après la freondaliom. lat partir supririeure du spadice disparaît; ainsi que la spathe. L'axe d'inflorescence, surmonté des débris de celle-ci, porte un petit amas subsphérique defruits. Ceux-ci sont des baies, à peine charnues, conservant la forme de l'ovaire, surmontées des dibris du style, indrihiscentes. Les graines, au nombre de six environ, sont ovoïdes aigues, rugueuses longiludinalement, a funicule obeonigue, court, munie d'une strophiole (arille; plus longue et plus large du double que le funicule; elles renferment, au milieu d'un albumen charnu, un petit embryon, cylindrique, droit, à radicule quelque peu épaissie.

Les dimensions moyennes de la plante sont:

Feuille de la plante adulte.

Pétiole.

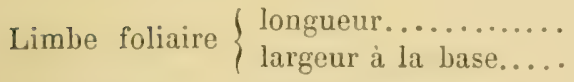

$20-230 \mathrm{~cm}$

lobes supérieurs lobes inférieurs

$5-6 \mathrm{~cm} \quad 2-3^{\mathrm{cm}}$

$6-7 \mathrm{~cm} \quad 3-3 \mathrm{~cm}, 5$

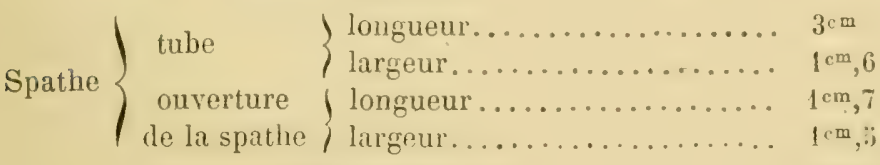

\begin{tabular}{|c|c|c|}
\hline & & \\
\hline ice & 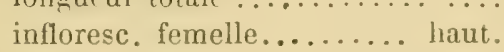 & $0 \mathrm{~cm}, 3$ \\
\hline & - mâle......... - & $2^{\mathrm{m}}, \mathrm{b}$ \\
\hline upposé rectiligne) & manche............. long. & $2^{\mathrm{cm}}, \ddot{\mathrm{j}}$ \\
\hline & massue...$\ldots \ldots \ldots \ldots$ - & $1^{\mathrm{cm}}$ \\
\hline & hauteur. & $1^{\mathrm{mm}}, ;$ \\
\hline Ovaire & 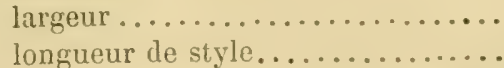 & $\begin{array}{l}2^{\mathrm{mm}}, 5 \\
2^{\mathrm{mm}}, 0\end{array}$ \\
\hline
\end{tabular}

Ovule $\ldots \ldots \ldots \ldots \ldots \ldots$ haut. $0 \ldots \ldots$ mm $, 7-0^{\mathrm{mm}}, 9$

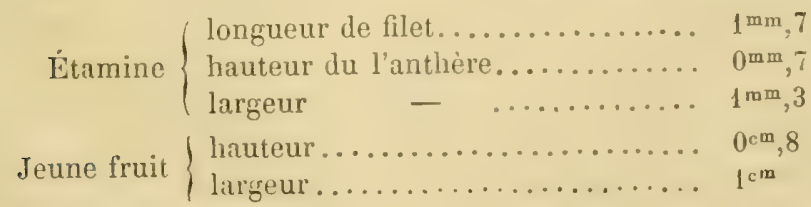


Rem. Dans ceite espèce, la forme du limbe foliaire est quelque peu variable, méme sur les divers individus d'une mème localité; les lobes postérieurs sont plus ou moins longs, plus ou moins réfléchis en arrière et en dehors, le limbe de la spathe est aussi d'une longueur un peu variable; le spadice plus ou moins exsert, à massue plus ou moins renflée.

Les diverses formes ou variétés assez distincles, établies sur ces variations des organes végétatifs et reproducteurs, décrites par les auteurs, ne se rencontrent point dans notre pays; il serait sans intérêt d'y insister ici.

Distrib. GÉographiQue. - Arisarum vulgare est une plante répandue dans toute la région méditerranéenne, et qu'on ne rencontre en France que dans̀ nos départements méridionaux de cette région.

\section{$2^{\circ}$ Caractères anatomiques $(1)$.}

Tubercule (portion âgée). - Epiderme partout exfolié par une assise subéreuse, colorée en jaune, différenciée à une faible profondeur dans le parenchyme périphérique, assise formée d'éléments aplatis, peu sinueux, régulièrement alignés en files radiales (8-9 assises concentriques environ), à parois minces, nettement mais faiblement colorables (vert d'iode). Des plages de parenchyme mort, à éléments vides, adhèrent par endroits à la face externe de l'assise subéreuse.

La masse du tubercule est formée d'un parenchyme homogène, ¿̀ éléments obovales ou faiblement polygonaux, en partie comprimés et rompus dans la zone périphérique, à parois minces, sans méats.

Examiné vers la fin de la période de végétation, le tubercule se montre rempli de grains d'amidon, de taille inégale, mais toujours pelits, isolés ou plus souvent groupésen petit nombre(2-3-4), à face externe convexe, à faces latérales accolées, polygonales et plines.

Dans tout le rhizome, mais.avec plus d abondance à la périphérie,

(1) L'étude anatomique de l'Arisarum vulgare n'avait pas été faite jusqu'ici. 
existent de larges biments, avec gros parfuets de raphides d'oxalate de chaux. La plupart de ces éléments raphidifères ont leur paroi épaissie.

Dautres cristaux, formes vaisemblablement anssi doxalate de chaux, sont localisis par potits groupes dans des bluments spreciaux, épars dans tout le parenchyme. Chature groupe est formí d'un petit

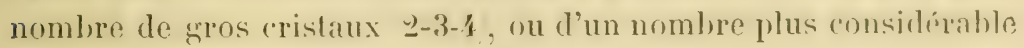
(7-8) de cristaux de plus petile laille; de très petits ristaux, aceompagnent toujours les cristaux plus volumineux. Ces cristaux se présentent sous forme de tables quadrangulaires, à angles tronqués, ou de prismes d'apparence puadratique, avec facettes anx angles, les plus petits revêtent la forme de petites tables rectanculaires, ou de petits prismes, avec pointements aux deux bouts. Les éléments qui contiennent ces cristaux ont leur paroi moins épaissie que celle de la plupart des éléments raphidifères.

Les vaiseaux cribro-vasculaires, nombreux, ramifiés dans toute la masse du tubercule, et lans toutes les directions, ont leurs éléments vaseulaires, uniquement spiraiés, facilement déroulables, longs, lignifiés, à section polygonale, de faible calibre.

Radicelle adventive (struct. primaire). La coupe transversale est sinueuse, irrégulière à la périphérie.

L'écorce se divise naturellement en 2 portions très distinctes: a. l'externe, $b$. l'interne, à peu près de même épaisseur.

La portion externe $a$ se divise elle-même en 3 zones :

$1^{\circ}$ Zone externe, réduite à deux assises de cellules: l'assise la plus externe, forméc de cellules mortes, ì section rectingulaire, à parois minees, l'externe lignifiée, l'assice interne, à élements superposés à ceux de l'assise précédente, à parois non lignifiées.

2 \%one interne à éléments morts, comprimés, irrégulièrement tassés, à parois minces; zone séparée elle-même en 2 couches par de larges lacunes aérifères irrégulières.

$3^{\circ}$ Zone interne à éléments morts, dont les parois minces, comprimées et (de ce fait sinueuses, plissées, ont perdu leur adherence avec les parois des éléments voisins.

La portion interne $b$ est formée uniformément de $2-3$ couches, 
de sriands déments, de dimensions décroissantes a mesure que l'on se rapproche du centre, lous alignés dans le sens radial, ceux de la couche externe ovales-allongés, ceux de la couche interne ovales el courts.

Cerlains éléments de cette assise (soumis à la pression due ì l'accroissement en épaisseur de cette zone), ont leur paroi détachée de celle des éléments qui les entourent, cette paroi apparaît alors avec d'élégantes spirales, dues sans doute au plissement qu'elle subit.

Endoderme trìs net, à éléments ob-quadrangulaires à la section, lignifiés, à paroi radiale tout entière épaissie.

Les éléments de l'assise péricyclique ne se distinguent que par leur laille un peu plus considérable de ceux qui constituent la masse conjonctive du cylindre central. Les faisceaux libériens, peu distincts (sur 'a coupe transversale), du parenchyme ambiant, occupent tout l'intervalle qui sẻpare 2 faisceaux ligneux voisins.

Ceux-ci, au nombre de 6 (2 voisins souvents confluents), à pointe externe, sont formés de vaisseaux lignifiés, à section sub-circulaire, ou faiblement polygonale, les plus jeunes et les plus larges vers le centre.

Feuille $n^{\circ} 1$ (pię âgé). - Pétiole. (La très grande analogie qui existe, quant à l'agencement des faisceaux, entre l'Arisarum et les Arum, nous dispensera d'insister longuement sur ce point.)

C. initiale (à mi-hauteur de la portion vaginale du pétiole) en fer à cheval, un peu irrégulier, à branches aiguës, l'une chevauchant sur l'autre (l'extérieure plus grêle); faisceaux (20 environ) à bois interne, sur plusieurs ares, plus ou moins régulièrement concentriques.

C. médiane (à la pointe de la gaine) irrégulièrement elliptique, devient rapidement sub-circulaire à un niveau supérieur; les faisceaux, voisins du centre, cessent d'ètre assez régulièrement distribués sur des arcs concentriques.

C. terminale (ou caractéristique) (à la naissance du limbe) obtriangulaire, à angles obtus (les supérieurs renflés pour former l'ébauche du limbe), à concavité supérieure peu accentuée; faisceaux péri- 
phériques, distribucis avee riogularite sur @ ares, laremement ouverls du cóté supérieur.

Nerv. médiane du limbe (c. au milieu) convexo-uncave, à dépression supérieure médiane; ว̌ faisceaux collenchymaleux; 7 faisęaıx cribrovaseulaires, tous à bois superieur, $\unlhd$ infiriens, $\unlhd$ supricurs, et 3 sur un arc médian.

Lal coupe transversale, à ces divers niveaux, presente foujours mon: face externe, légèrement onduleuse; chaque ondulation est presifue régulièrement déterminée par les saillies d'un faisceau de collenchyme, faisceau placé au dos, ou supeposé à peu de distance, d'un des faisceaux cribrovasculaires périphériques.

A tous les niveaux, on constate:

Epiderme à éléments quadrangulaires sur la c. transvers, un peu plus petits en face des massifs de collenchyme, à parois minces, sauf l'externe, fortement epaissie, arec cuticule ì stries difficilement perceptibles, à stomates peu nombreux, localisés aux creux des cannelures, semblables à ceux du limbe; les cellules superposées aux saillies des cannelures sont plus nettement rectangulaires, et plus allongées que celles qui tapissent les creux des mêmes cannelures.

Les faisceaux collenchymateux, immédiatement sous-jacents à l'épiderme, sont formés d'éléments allongés, à paroi épaissie, surtout aux angles; ils existent dans toute la longueur du pétiole el de la nervure médiane. L'exoderme est peu distinct du reste du parenchyme sous-jacent. Le parenchyme cortical, à éléments obovales, réguliers, de petites dimensions, à nombreux méats, est nettement distinct du parenchyme central, à éléments plus larges et plus irréguliers. 11 n'existe pas de vraies lacunes aérifères; mais çà et là, dans la région centrale, les éléments parenchymateux seécartent; leurs parois ondulées, souvent rompues, limitent des carités irrégulières qui, par confluence plus ou moins complète, rendent le centre de l'organe irrégulièrement fistuleux.

Cette tendance à la formation de cavités s'ébauche dans la portion vaginale du pétiole, s'accentue en son milieu, et disparaît rers le haut. A ce dernier niveau, ainsi que dans la nervure médiane, on 
tronve de vraies lacunes aérifères, régulières, limitées par des files uniques de cellules, identiques à celle du pétiole des Arum.

Sur les corps tout à fait supérieures (alors que le limbe est déjà accusé sous forme de deux lames latéro-supérieures), on voit une lacune centrale, tapissée par un épiderme nel; cette figure est due à une véritable invagination du sinus de la nervure médiane du limbe, à une faible profondeur, dans la masse centrale de la portion tout à fait terminale du pétiole.

Limbe. - Êpiderme inférieur à éléments ondulés, allongés audessus du trajet des nervures, à cuticule lisse et mince. Epiderme supérieur présentant les mêmes caractères, mais à éléments un peu plus grands, moins onduleux, à cuticule un peu plus mince, paroi munie de petits épaississements, à disposition moniliforme. Stomates présents sur les deux faces, inais bien moins nombreux à

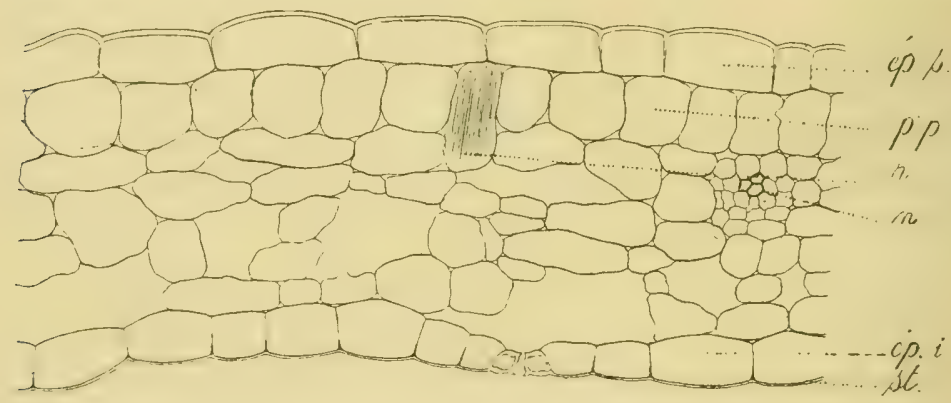

Fig. 36. Arisarum vulgare T. T. (Gross. 220/1). - Limbe foliaire (c. transvers.).

la face supérieure, obovales dans l'ensemble, accompagnés de deux (rarement de trois à quatre) cellules annexes latérales, convexoconcaves, la face externe des cellules stomatiques surplombe, en se rabattant en dedans, l'ostiole; leurs deux lèvres externes, incisées, limitent ainsi une petit chambre sus-ostiolaire.

Le mésophylle est unifacial, il comprend une seule assise de larges el courts èléments palissadiques, çà et là différenciés en cellules raphidifères, et un mésophylle inférieur, à éléments irréguliers, la plupart aplatis tangentiellement, à larges lacunes. Les nervures, isolées dans le mésophylle, à mi-distance à peu près de 
deux faces, ne sont entourées que d'une gaine de parenchyme condensé, à petils éléments, el n’unt que des ćléments vatsculaires spiralés.

Les éléments du mésophylle renferment des masses mucilagineuses, identiques à celles déjà signalées chez les Arum.

Rem. - On ne trouve pas, chez Arisarum vulyare, de cellules à contenu résineux, analogues à celles qui sont éparses, en si grande abondance, sur les divers organes des Arım.

Nous n'étudierons pas la structure analomique de l'inlloresence et des fruits, ces organes n'auront jamais à être déterminés microscopiquement par le médecin. Le fruit, vert el presque sec, ne sera jamais consommé par mégarde, pas plus que la spathe dont la forme et la couleur bizarre suffisent à éviter toute méprise.

Outre les ouvrages généraux sur les Aroülées, el les flores locales signalées plus haut (v. p. 14-46), Voir:

Bibl. 1719. - Tounnefont (J.-P. de). Institut. rei herbarix (131, tab. 70).

1797. - JAGQUIN (N-.J.). Plant. rar.hort.Schonbrunn... (II, p. 64), t. 192 (Sabb. IIort. II, t. 79. Barr. Ic. t. 573).

1813. - Sibthonp (J.). Flora græea (II, p. 24:̈).

1762-63. - Linné (C.). Species plantarum (II, p. 1370).

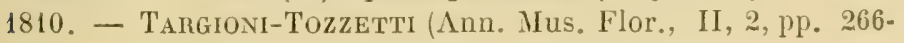
$617)$.

1832. - Schotт (H. W.) et Endicher.(S.). Meletemata botanica $(\mathrm{I}, 516)$.

1833. - Richard (L.C.) (in Guillemin Archiv. Jot. III, 20, t. 2).

1834-70. - Reichenbach (II. G. L. et G. II.). Icones Floræ Germanicx (VII, t. 7).

1835. - Blume (K. L.). Rumphia (I, pp. 89-90).

1836. - Endicher (S. L.). Genera plant. (n. 1673, p. 234). Kunth (K. S.). (Mém. Mus. IV, p. 438).

1841. - „ Enumerat. plant. (III, p. 14-15).

1852. - " Floraitaliana (II, p. 234-5).

1855-1856. - Grenier (A.) et Godrox (D.A.). Flore de France (III, 331).

1856. - Parlatore (F.). Note sur l'Arisarum (Bull. Soc. bot. de France, p. 341).

1856. - Schotт (H. W.). Synopsis Aroïdearum (p. 4).

1860. - Schotт (II. W.). Prodr. Syst. Aroïdearum (p 20). 
ICON. 179\%. - JACQUIN (N.-J.) Op. rit. (t. 192).

1840. - Sibthone (J.). Flora græca (tab. color, 9'8).

Explor. scient. de l'Algérie (tab. 44, (fig. 10-12).

Explication de la Pl. 11 .

Arisarum vulgare T'ARg. Tozz.

FIG. 1. - Rhizome, avec bourgeon latéral (la pousse feuillée coupée)...

GRoss,

2. - Portion rhizomateuse, renflée en bulbe l'année précédente, et émettant un bourgeon latéral (la pousse feuillée de l'année coupée $\ldots \ldots \ldots \ldots \ldots \ldots \ldots \ldots \ldots \ldots \ldots \ldots \ldots \ldots \ldots \ldots \ldots \ldots$

3. - Inflorescence un peu avant l'anthère................. $\frac{1}{1}$

4. - Inflorescence au moment de l'anthère................. $\frac{1}{1}$

5. - Inflorescence après l'anthère, la spathe fendue........... $\frac{2,5}{1}$

6. - Fleur femelle (ovaire) isolée.................... $\frac{13}{1}$

7. Autre fleur femelle (coupe longitudinale).............. $\frac{13}{1}$

8-9-10-11. - Diverses formes d'ovules................. $\frac{15}{1}$

12-13. - Fleur mâle (étamine) (face interne et face externe)........ $\frac{22}{1}$

14. - Jeune fruit............................. $\frac{1}{1}$

Explication de la Pl. III.

\section{Arisarum vulgare TARG. Tozz.}

Fig. 1. - Jeune plante issue du développement d'un bourgeon latéral du rhizome $(1$ re année de végétation) $\ldots \ldots \ldots \ldots \ldots \ldots \ldots . \ldots \ldots \ldots \ldots$

2. - Plante adulte............................... $\frac{1}{1,5}$

3. - Tubercule sans bourgeon latéral (pousse feuillée coupẻe).... $\frac{1}{1}$

4. - Tubercule avec bourgeon latéral en voie de développement... $\frac{1}{1}$

5. - Tubercule avec bourgeon latéral en voie de développement

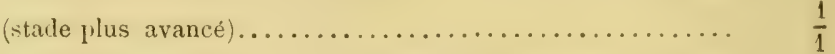

6. - Bourgeon latéral isolé (1 ${ }^{\text {re }}$ année de végétation) (pousse

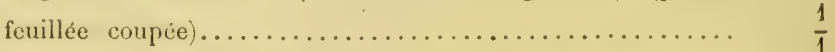

7. - Portion inférieure d'un vieux tubercule, montrant la séparation de la partie la plus âgée flétrie................ $\frac{1}{1}$

8. - Portion souterraine, purement rhizomateuse, d'un vieux pied..

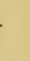




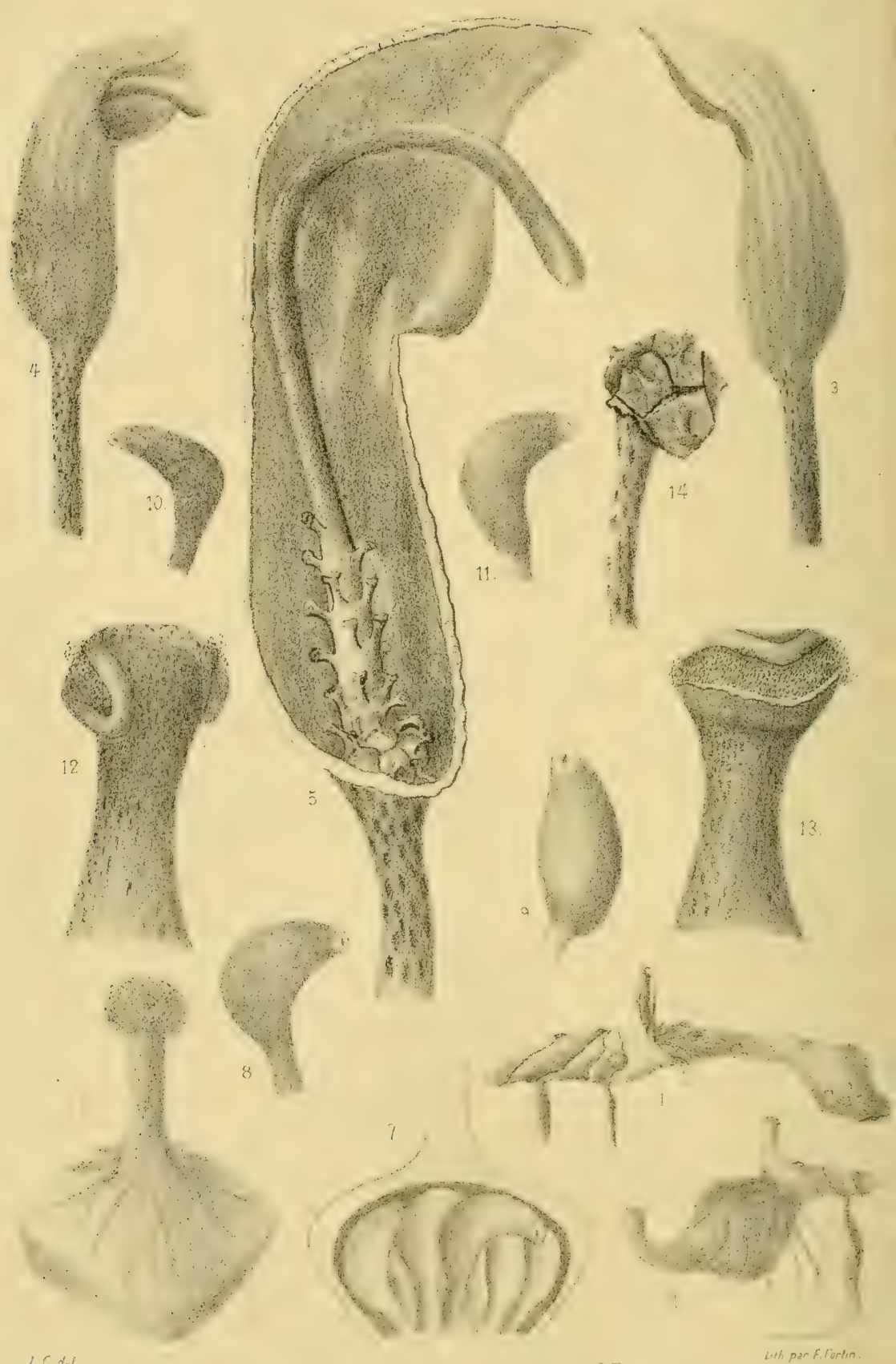

sarum vilgaré 'IARG.TOZ. 
P1.111

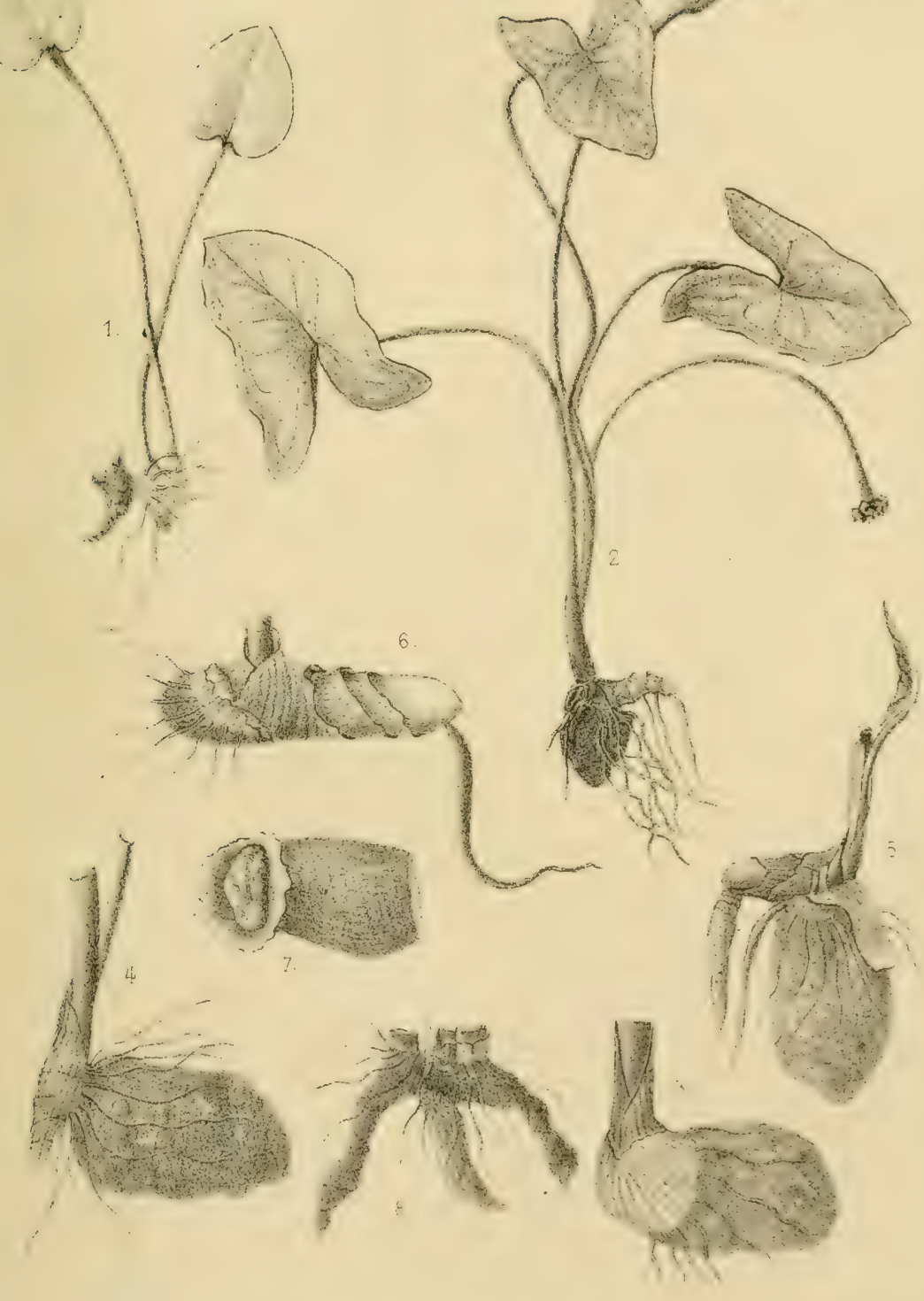

IC.del Arisd uis $7 \cdots$ a 



\section{CHAPITRE II}

\section{ÉTUDE GHIMIQUE}

Les études poursuivies jusqu'à ce jour sur les principes chimiques, contenus dans les Arö̈dées indigines, sont peu nombreuses, et les resultats auxquels elles ont conduit, la plupart incertains. Les ancirnnes analyses des tuberules d'Arum n'indiquent que la présence d'eau, de gomme, d'un acide, de sucre non cristallisable, de fécule et de ligneux. Le principe âcre était indiqué comme soluble dans l'eau. On a signalé, dans ces trente dernieres années, chez diverses plantes de cetle famille, la présence de $1^{\circ}$ de saponine, $2^{\circ}$ d'une base volatile indétermincé, $3^{\circ}$ d'une faible quantité d'acide cyanhydrique. Nos recherches ont eu pour but de vérifier la présence de ces trois corps, et dè les caractériser d'une facon plus précise qu'on n'avait pu le faire avant nous. Nous avons soumis à l'analyse les Arum maculatum et italicum, et l'Arisarum vulgare.

\section{A. - Arum maculatum et italicum.}

\section{ANALYSE ÉLÉMENTAIRE DES DIVERS ORGANes Des Arum}

Méthode d'analyse (1). - La méthode empluyée pour l'analyse élémentaire des principes, susceptibles d'exister dans ces plantes, est, à peu de chose près, celle proposée, pour l'analyse élémentaire des végétaux en général, par DragendorfF et SchlagdenhaUfFen (2).

Une quantité, relativement minime de l'organeà analyser, est broyée puis épuisée successivement par une série de dissolvants: 1. ligroine, 2. éther absolu, 3. alcool absolu, 4. eau distillée, 5. lessive faible de soude, 6. solution étendue d'acide chlorhydrique, 7. eau chlorée. Chaque dissolvant fournit par évaporation un extrait sec.

1. Ligroine (éther de pétrole).

(1) Il est à peine besoin de faire remarquer que cette méthode est tout à fait insuffisante pour nous renseigner sur la nature des hydrates de carbone contenus dans les tissus analysés. Mais ce point spécial ne présentait aucun intérêt médical.

(2) Dragendorff et Sumlagdeniaurfex. Encyclopédie chimique. Analyse chimique des végétaux. 1882. - DragendonfF (G.). (Die qualilative und quantitative Analyse von Pflanzen und Pfianzentheilen, Güttingen. 
$\begin{array}{cl} & -62- \\ \text { L'extrait sec peut contenir } & \left\{\begin{array}{l}\text { huiles essentielles, } \\ \text { graisses, } \\ \text { matières cireuses, } \\ \text { chlorophylle(entrainée par les graisses). } \\ \text { alcaloïdes, }\end{array}\right. \\ \text { On peut séparer les } & \left\{\begin{array}{l}\text { huiles essentielles par distillation, } \\ \text { graisses par l'alcool fort, puis l'évapo- } \\ \text { ration (carac. tache le papier, odeur } \\ \text { d'acroléine). }\end{array}\right.\end{array}$

2. Ether absolu (à $66^{\circ} \mathrm{B}$, exempt d'alcool et d'eau) (anhydre, ne dissout plus les tanins).

Le résidu de l'épuisement par la ligroïne, après lavage et dessiccation, est mis à macérer pendant 7 à 8 jours dans l'éther.

L'extrait est évaporé à l'air, puis au-dessus de $\mathrm{SO}^{4} \mathrm{H}^{2}$.

$$
\text { Il peut contenir } \quad\left\{\begin{array}{l}
\text { chlorophylle, } \\
\text { alcaloides, } \\
\text { acide gallique, } \\
\text { résines. }
\end{array}\right.
$$

Alcaloïdes caractérisés à l'état de sels.

Une partie de l'extrait sec pulvérisé est traitée par l'eau distillée; la solution aqueuse doit contenir l'acide gallique, s'il en existe dans l'extrait.

La partie insoluble dans l'eau est traitée par l'alcool absolu qui dissout les résines et la chlorophylle; on se débarrasse de la chlorophylle par la benzine; les résines sont solubles dans une solution alcaline, d'où l'acidulation par $\mathrm{SO}^{4} \mathrm{H}^{2}$ les précipite.

\section{Alcool absolu.}

Le résidu de l'épuisement par l'éther est mis à macérer pendant 7 jours dans l'alcool.

$$
\text { L'extrait alcoolique sec peut }\left\{\begin{array}{l}
\text { tanins, } \\
\text { alcaloìdes, } \\
\text { glucosides, } \\
\text { glucose (petite quantité), } \\
\text { phlobaphènes (provenant de la décom. } \\
\text { position des tanins) } \\
\text { matières colorantes, } \\
\text { certains sels. }
\end{array}\right.
$$


L'extrait sec est épuisé par l'eau distillée chaude.

On y caractérise les tanins

$$
\begin{aligned}
& \text { précipitation par chlorure ferrique, } \\
& \text { précipite la gélatine, } \\
& \text { rar refroidissement un précipité se } \\
& \text { forme, soluble dans l'alcool (cette } \\
& \text { précipitation par refroidissement } \\
& \text { rend presque impossible l'épuisement } \\
& \text { de l'extrait par l'eau chaude; le ré- } \\
& \text { sidu de cet épuisement contient en- } \\
& \text { core des tanins). }
\end{aligned}
$$

La solution résultant du traitement par l'eau est acidulée par $\mathrm{SO}^{4} \mathrm{H}^{2}$, puis agitée avec : 1. éther de pétrole, 2. benzine, 3. chloroforme. Chacune des trois solutions est mise à déposer (le dépôt peut être dû à des alcaloïdes ou à des glucosides).

La solution aqueuse est débarrassée des tanins, alcaloïdes, glucosides, par précipitation avec l'acétate de plomb; on peut ensuite y caracteriser les sucres.

Le résidu de l'épuisement par l'eau est épuisé par l'eau ammoniacale $\left(1 / 5_{50}\right)$. Dans cette solution colorée, les tanins précipitent par l'acide acétique. Le résidu est formé de phlobaphènes, le résidu de ce dernier traitement est épuisé par l'eau acidulée; cette solution peut contenir des alcaloïdes.

\section{Eau distillée.}

Le résidu de l'épuisement par l'alcool est épuisé, pendant 48 heures, par l'eau distillée ( $10^{\mathrm{c} 3}$ pour $1 \mathrm{gr}$. de résidu).

tanins,

alcaloïdes,

sucres (saccharoses, glucoses),

L'extrait aqueux peut acides végétaux,

contenir

matières colorantes,

matières pectiques,

matières albuminoïdes,

sels minéraux.

5. Lessive faible de soude ( $3 \%)$.

Le résidu de l'épuisement par l'eau subit dans la lessive trois macérations successives de 24 heures.

L'extrait alcalin peut contenir.

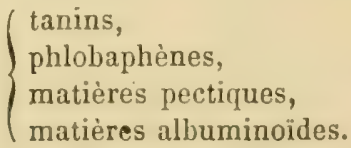


6. 7. Acide chlorhydrique. - Eau chlorée. - L'épuisement final par IICl étendu et par l'eau chlorée, ne présente aucun intérêt, à notre point de vue spécial, tous les principes actifs ayant été enlevés par les épuisements précédents.

L'analyse élémentaire des divers organes, par la méthode cidessus indiquée, nous a donné les résultats suivants :

Rhizome (pendant la période de végétation, arril-mai).

Arum maculatum.

Arum itaticum.

1. Ether. Graisses (peu).

Id.

2. Alcool.

Alcaloïdes (ppté par KI iodé, conicine?).

Matières minérales (peu).

Tanins (peu).

Principes réducteurs.

Résine (traces?).

3. Eau. Sels minéraux.

Matières albuminoïdes.

- pectiques (?).

Tanins (traces).

Sucres réduits (peu).

4. $\mathrm{NaOH}(3 \%)$. Matières pectiques(?).

- albuminoïdes.

Tanins (peu).

5. HCI $(1 \%)$. Matières minérales.

Hydrates de carbone dédoublés (peu).

6. Résidu. Cellulose.

Feuilles. Arum maculatum.

Arum itaticum.

1. Ether. Graisses (peu).

Id.

2. Alcool.

Alcaloïdes (ppté par KI iodé, conicine?).

Matières minérales.

Tanins. 
Principes réducteurs.

Résine (traces?).

3. Eau.

Sels minéraux.

Matières albuminoïdes.

- pectiques (?).

Tannins.

Sucres réduits.

4. $\mathrm{NaOH}(3 \%)$. Matières pecliques (?).

- albuminoïdes.

Tannins.

כ. $\mathrm{HCl}(\mathbf{1} \%)$. Matières minérales.

Hydrates de carbone dédoublés (peu).

6. Résidu. Cellulose

Fruits (péricarpe et yraine) (à maturité, fruits rouges). Arum maculatum.

1. Éther. Natières colorantes.

Arum itaticum

Graisses (peu).

2. Alcool. Alcaloïdes (ppté par KI iodé, fume avec $\mathrm{IICl}$, conicine (?).)

Natières colorantes.

- minérales (peu).

Tannins.

Principes réduits.

Résine (traces?).

3. Ean.

Sels minéraux.

Albuminoïdes.

Natières pectiques (?).

Tannins (traces).

Sucres réduits (peu).

4. NaOH $(3 \%)$. Matières pectiques (?).

Albuminoïdes.

Tannins.

5. HCl $(1 \%)$. Matières minérales.

Hydrates de carbone dédoublés(peu).- 
6. Résidu. Cellulose.

Fruits. Péricarpe (isolé) (à maturité).

La recherche des glucosides et alcaloïdes est seule pratiquée; résultat positif.

Graines (isolées).

Arum maculatum.

1. Ether. Matières colorantes (peu).

Arum itaticum.

Acide gallique.

Graisses (traces).

2. Alcool. Alcaloïdes (peu).

Matières colorantes (très peu).

- minérales.

Tannins.

Sucres réduits.

Glucosides.

3. Eau.

Matières minérales.

Albuminoïdes.

Tannins.

Sucres réduits (traces).

- non réduits (très peu).

$4 \mathrm{NaOH}(3 \%)$. Albuminoïdes.

Tannins.

5. HCI (1 \%). Amidon (beaucoup).

Matières minérales.

Acide gallique.

Hydrates de carbone dédoublés (abondant).

6. Résidu. Cellulose.

Ligneux.

$I d$.
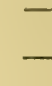
quantité suffisinte pour les caracterisir nctlement, et contrôler les assertions de nos devanciers.

SAPONINE:

La présence de la saponine, dans les tubercules d'Arum maculatum, a d'abord été signalée par Evz, confirmée par Srics qui la retrouve dans $A$. itulicum, puis par IVAAGE, qui dit avoir isolé de la saponine des $A$. maculatum, italicum, Dioscnidis.

La marche indiquée par Exz pour l'extraction de la saponine, en partant des tubercules, est la suivante:

Les tubercules (rhizomes) frais d'Arum maculatum, traités par l'espritde-vin, donnent une teinture. Cette teinture est distillée, filtrée, puis on évapore la liqueur restant dans la cornue. On traite le résidu par l'alcool bouillant et on évapore.

L'extrait alcoolique est repris par l'eau; on ajoute à la liqueur de la levure de bière bien levée, qui y détermine une vive fermentation.

Lorsifue la fermentation s'arrête, on filtre, on évapore et on reprend par l'alcool.

La solution alcoolique fournit de la saponine, sous forme de petits cristaux grenus, d'une saveur d'abord douce, puis amère et âcre.

Pour notre part, nous avons suivi, en vue de l'extraction de la saponine, la marche ci-dessous.

Les plantes, séchées et réduites en poudre, sont traitées par l'alcool bouillant à $90^{\circ}$. Après quelques minutes d'ébullition, on filtre; par le refroidissement, la saponine se précipite en partie; on filtre le liquide. Le précipité est filtré, desséché dans le vide. On réitère le traitement jusqu'à épuisement de la plante.

Les liquides alcooliques qui sont colorés en jaune contiennent encore une certaine quantité de saponine. Evaporés à siccité au bain-mariedans le vide, ils fournissent un extrait qui, traité par l'alcool étendu bouillant, donne une nouvelle qquantité de saponine.

La saponine obtenue est purifiée par des lavages à l'éther.

Le dépot blanc, obtenu par refroidissement de la solution alcoolique, filtrée et séchée, donne une solution aqueuse qui reste trouble, même après filtration, et mousse abondamment. Après interversion par $\mathrm{SO}^{4} \mathrm{H}^{2}$ etendu, la solution aqueuse réduit énergiquement la liqueur de Fehling et laisse un résilu résinoide insoluble, qui correspond rraisemblablement a la sapogénine. 
Lil quantilé la plus considirable de saponine que nous avons pu extraire fdes Armm nous a été fournie par les tubercules, à la fin de la période de végétation (juin-juillet), ałors que les fruits commencent à mûrir et l'axe d'inflorescence a été privé de toute connexion avec le tubercule, par suite de la désorganisation de ses tissus à la base. La teneur maxima des tubercules d'Arum en saponine a été à peu près de $1 \%$ de substance fraîche.

La saponine n'existe d'ailleurs pas en quantité à peu près constante, et à beaucoup près, dans les organes de végétation des Arum; l'expérience suivante le prouve.

5. kilos de tubercules d'Arum italicum en état de repos hivernal, expédiés du midi de la France, sont mis en végétation dans du sable humide infertile en serre, aux premiers jours de février; quinze jours après, ils ont épanoui deux ou trois feuilles vertes, les parties aériennes sont alors séparées des parties souterraines; ces dernières donnent, après dessiccation et pulvérisation, 520 grammes de poudre; cette poudre traitée par l'alcool bouillant (en vue de l'extraction de la saponine), ne donne, après refroidissement, que de très légers flocons qu'il est impossible de recueillir sur un filtre. On fait alors quatre décoctions successives de trois litres d'alcool à chaque fois, toutes les liqueurs sont réunies et distillées, le résidu de la distillation repris par un litre d'alcool bouillant. Cet alcool, pas plus que les décoctions précédentes, ne laisse déposer de saponine; on ajoute à cette solution alcoolique un volume d'éther égal à celui de l'alcool; cette addition d'éther précipite un produit mielleux dans lequel nagrent quelques cristaux (sels minéraux de potassium et calcium (chlorures?). Ce liquide sirupeux ne contient pas trace de saponine, il renferme une quantité très faible d'un principe réducteur (de la liqueur de Fehling).

Donc les tubercules d'Arum peuvent, au moment de la reprise de la végétation, avant l'époque où l'assimilation chlorophyllienne est intense, être totalement privés de saponine; par contre, les parties vertes, récemment épanouies, contiennent une cerlaine quantité de ce même corps.

De là à admettre que la saponine migre au printemps des parties souterraines vers les parties aérieunes en voie d'épanouissement, il n'y a qu'un pas. C'est un fait que nous proposons d'élablir ullérieurement par des essais plus suivis. 
La saponine d'Armm est-celle susceptible d'étre dedoublie, dans les tissus qui la renferment, par un ferment soluble, comme e'est le cas pour de nombreux glucosides végélaux?

Nous avons, pour répondre à cette question, procédé à la recherche d'une diastase:

$1^{\circ}$ Dans les tubercules (à la reprise de la végétalion).

On fait digérer les tubercules hachés dans l'eau à $330-40^{\circ}$, pendant plusieurs heures, on divise le liquide en 2 portions : la première précipitéc par l'aleool, la seconde additionnée de phosplıate d'ammoniaque et précipitée par le chlorure de calcium.

Ces précipités sont filtrés et séchés dans le vide.

On dispose les expériences suivantes :

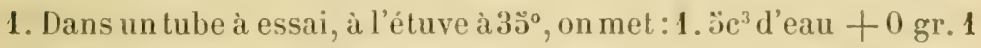
desaponine (du commerce, du bois de Panama) + précipité par l'aleool.

2. Même expérience après ébullition, your détruire le ferment soluble supposé.

3. Même expérience que 1, mais avec précipité par le phosphate. de chaux.

4. Nême expérience que 3 , mais après ébullition.

Tous ces essais donnent un résultat négatif.

L'expérience sera à reprendre avec de la saponine d'Arum, et-à d'autres périodes de la végétation.

$2^{\circ}$ Dans les pousses vertes (émises par les mêmes tubercules).

Mêmes essais, mème résultat négatif, avec le phosphate de chaux seulement, la proportion d'albuminoüdes contenus dans ces jeunes pousses était si faible qu'il.n'y a eu qu'un louche par l'alcool.

Il semble ne pas y avoir dans la plante, lors de la reprise de la végétation, de ferment soluble capable de dídoubler la saponine.

Le temps et les matériaux nous ay̧ant manqué pour extraire, pendant la période de repos des tubercules, une quantité notable de saponine, il nous a été impossible d'essayer de caractériser, par dés réactions colorantes, la saponined'Arum. C'est une lacune à compléter. Yous ne pourons donc aflirmer, dès maintenant, lidentité uu 
la distinction de cette saponine avec les nombreux homologues, découverts dans une foule de plantes, et dont beaucoup doivent, en dépit des noms différents imposés prématurément par leurs obtenteurs, être identiques.

Les propriétés de:

$1^{\circ}$ rendre l'eau très mousseuse;

$2^{\circ}$ réduire, après interversion facile, la liqueur de Fehling;

$3^{\circ}$ laisser, après interversion, un résidu résinoïde, vraisemblablementidentique à la sapogénine.

Nous paraissent suffisantes pour classerle glucoside d'Arum dans le groupe des saponines.

On sait (V. Bibl. ci-dessous) qu'il existe tout un groupe de glucosides, formant une série d'homologues, et que l'on peut désigner sous le nom de groupe chimique de la saponine,

Deux groupes secondaires sont cependant à distinguer dans ce grand groupe : a) un groupe neutre, insoluble dans l'alcool, dont la sapotoxine du Quillaja est le type;

b) un groupe acide, soluble dans l'alcool, dont l'acide quillajique est le type.

Par sa solubilité très grande dans l'alcool, la saponine des Aroïdées appartient à ce second groupe.

Les propriétés organoleptiques de toutes les saponines sont les mêmes. Ce sont des substances amorphes, incolores, jaunâtres, solubles dans l'eau et la faisant mousser fortement.

Nous nous promettons d'établir, après essais plus fructueux d'extraction, les qualités particulières ì la saponine d'Arum, et ses rapports avec les saponines actuellement bien définies.

Bibl. 1874. - Christophson. Unters, über d. Saponin, Diss. Dorpat. 1882. - Leque (Marius). De la Saponaire et de la Saponine, Thèse, Ec. pharmacie, Paris.

1886: - DragendonfF. Beitr. zur ger. chemic. - Manuel de Toxicologie, trad, franc., p. 205, 214, 242.

1887. - Kobert (R.). Ueber Quillajasaüre (Arch. f, exper. Pathol. und Pharmakol. Bd. 23, p. 233).

1888. - ATLaSS (J.). Ueber Senegin (in Arbeit, des Pharmakolog. Institut. zu Dorpat.), p. 57-97.

1888. - Pachonukow (DmitriJ). Ueber Sapotoxin (ibid, p.1-51.) 
1888. - Tufanow (Nicolai). Ueber Cyulamin. (ibid., p. 100-142).

1891. - Kruskal (Nicolai). Ueber einige Saponinsubstanzen (ibid., p. 1-88.)

1891. - Kruskal (Nicolaï). Ueber Agrostemma Githago L. (ibid., p. 89-146).

1891. - Mohrberg (C.). Ueher Cephalantin (Das CephalanthusSaponine) (ibid., VIII, p. 34-35.)

1896. - Witold v. Schulz. Ein Reitr. zur Kenntniss der Sarsaparille (ibid., XIV, p. 1-78).

1896. - ID. Ein Beitr. zur Kenntniss einiger weiteren Saponin. substanzen, namentlich der rothen Seifenwurzel, ibid. (p. 82-113).

\section{ALCALOIJE}

Tous as auteurs sont d'accord à signaler chez la plupart des Arö̈dées, en particulier chez les A.um, la présence d'un principe volatil, facilement destructible, auquel ces plantes devraient, au moins en partie, leur âcreté.

Bird (d'après Husemaxix et Hilger) aurait isolé une base, facilement volatile, de couleur blanche et destructible avec la plus grande facilité.

Spraa se borne à signaler dans les $A$. italirum el maculatum une base volatile, sans rien préciser de sa nature.

\section{Méthode d'extraction.}

a) Parties aériennes, - 1 kil. 200 de plantes, un peu séchées à l'air, sont distillées avec 2 litres de potasse à $15 \%$; on recueille 1 litre à la distillation, d'où l'on peut extraire un demi-centimètre cube d'une base.

Cette extraction se fait de la façon suivante : On sature la distillation par $\mathrm{SO}^{2} \mathrm{H}^{4}$ étendu, on concentre dans le vide, on épuise le liquide concentré par l'éther, après y avoir ajouté un excès de potasse : l'évaporation de l'éther laisse la base comme résidu.

La proportion de cette base dans les parties vertes est extrêmement faible, un peu plus forte dans les plintes traitées à l'étal frais que dans celles qui ont subi un commencement de dessiccation.

100 kilos de parties vertes fraiches donnent environ 10 grammes d'une base impure. Nous pouvons évaluer, en moyenne, le rendement à $0,005 \%$ de plante fraîche.

b) Tubercules. - Le mème traitement appliqué aux tubercules frais permet d'extraire une quantité un peu plus considérable de cette base. 
Dans ces organes aussi, la proportion de cette base diminue a mesure que la dessication se produit.

c) Fruits. - Les fruits (péricarpe et graine), conservés dans l'alcool, sont réduits en pulpe et séchés. Le liquide de macération alcoolique est distillé dans le vide jusqu'à consistance sirupeuse.

On épuise par l'éther, le résidu a une forte odeur de souris; on le réunit à la pulpe des fruits séchés. On distille le tout au bain de sable avec de la potasse aqueuse: le liquide distillé, neutralisé par $\mathrm{SO}^{4} \mathrm{H}^{2}$, est évaporé, puis repris par l'alcool, qu'on évapore au bain-marie. Le résidu traité par $\mathrm{KOH}$, et épuisé par l'éther, développe une forte odeur de souris. Si la quantité de fruits traités est assez considérable, on obtient à la distillation des gouttelettes huileuses, lırunissant à l'air, et présentant l'odeur caractéristique signalée ci-dessus.

\section{Caractères.}

Nous obtenons donc ainsi une base impure, très peu soluble dans l'eau; sa solution aqueuse est alcaline sous forme d'un liquide brun, facilement altérable, de saveur âcre, soluble dans l'alcool, l'éther, la benzine, l'alcool amylique, le chloroforme, l'éther de pétrole, et communiquant à ces solutions une odeur caractéristique d'urine de souris.

Cette base présente tous les caractères d'un alcaloïde liquide volatil. En effet, sa réaction est alcaline; récemment préparée, elle répand des fumées, lorsqu'on en approche une baguette trempée dans HCl. En dissolution dans l'éther de pétrole, et après évaporation sur un verre de montre imbibé de $\mathrm{HCl}$, elle donne un dépôt faiblement cristallin, facilement déliquescent, répandant l'odeur de souris, facilement décomposable, soluble dans l'alcool. La solution aqueuse de chlorh ydrate cristallise en longues aiguilles; la base liquide dissoute par SO4 $\mathrm{II}^{2}$ étendu (à $\left.\frac{1}{30}\right)$, donne, en présence de l’iodure de potassium iodé, un abondant précipité brun, accusant ainsi sa nature alcaloïdique que confirme sa précipilation par les autres réactifs courants des alcaloïdes.

KI iodé est le réactif le plus sensible de la conicine; avec lui, la précipitation est encore distincte dans un liquide dilué au 1/8000, elle est encore risible dans une solution au $1 / 10000$ (limite de sa sensibilité\}. Aussi est-il le réactif auquel nous avons accordé la préférence, pour déceler l'alcaloïde, dans les divers extraits obtenus avec les organes des Arum. 
La solution sulfurirue de lia base se trouble par l'iodure double de bismuth et potassium.

Les deux principales hases organiques d origino végétale liquides, actuellement comnues, sont : la nicotine ta cicutine. On peut les distinguer par un cerlain nombre de caractères, spéciaux à la cicutine, et que ne présente point la nicotine. Ces caractères sontles suirants :

La cicutine $\quad\left\{\begin{array}{l}\text { rougit par } \mathrm{AzO}^{3} \text {; précipite en solution } \\ \text { aqueuse par l'eau chlorée à froid; est } \\ \text { moins soluble dans l'eau à chaud qu'à } \\ \text { froid; fume pn présence d'II'l; coa- } \\ \text { gule l'albumine; odeur de souris. }\end{array}\right.$

La base impure extraite des Arum présente les caractères regardés comme spéciaux à la cicutine. Les essais suivants le prouvent.

Une baguette de verre, trempée dans $H \mathrm{Cl}$, répand des fumées blanches, lourdes, épaisses, lorsquion l'approche de cette hase.

La solution aqueuse de celte base est suffisamment étendue pour ne présenter qu'un trouble faible. Sous l'influence de la chaleur, cette solution devient laiteuse et se clarifie par le refroidissement. Traitée par l'eau additionnée de $\mathrm{SO}^{4} \mathrm{H}^{2}$, elle donne un sel qui, par évaporation, apparait, vu au microscope, sous la forme de tablettes à contours nets, irrégulièrement hexagonales, avec quelques prismes très déliés, d’apparence plus ou moins vaguement quadratique. Le sulfate de cette base cristallise dailleurs mal, et ne tarde pas a tomber en déliquescence.

Une goutte de solution de cicutine (de ciguë) placée au contact d'une solution limpide d'albumine de l'oruf, la précipite. La formation de ce précipité est particulièrement nette en opérant de la façon suivante : Une goutte de solution aqueuse de cicutine est placée sur une lame de verre, au contact d'une goulte d'albumine pure. L'alcaloïde diffuse très rapidement dans la goutte d'albumine, et la zone de diffusion est indiquée, au fur et à mesure de sa progression, par une pellicule opaline de coagulation. Au microscope, cette pellicule se résout en une multitude de petits points 
brillants, correspondant à des granules dabumine precipitée /ces petits points ressemblent étonnamment aux fines pouctuations réfringentes qu'on observe sur les coupes du cartilage hyalin).

Gelte réaction répétée avec l’alcaloüde des Arum se produit arec moins de netteté, ce qui se concoit facilement, l'alcaloïde se trouvant mélingé à une quantité assez considérable de produits goudronneux. Pour la produire avec le plus de succès, le mieux est de recouvrir une goutte de la solution d'alcaloïde d'Arum par une large goutle d'albumine. Les produits goudronneux s'émulsionnent alors en fines goultelettes, et une zone dapparence laiteuse se dessine. Il ne s'agit point li d'une vraie coagulation. Chaque gouttelelte goudronneuse apparaît au microscope avec une teinte brune, l'alcaloïde a une zone de diffusion plus large que les produits goudronneux qui l'accompagnent. A jour frisant, la limite de cette zone est décélée par une zone laiteuse très pâle, qui est la véritable zone de coagulation.

Au microscope, cette zone se montre entièrement parsemée de petits granules brillants d'albumine précipitée, granules absents de part et d'autre rle celte zone. Si nous opérons avec une solution moins diluée d'alcalö̈de, ces petits granules épars se fusionnent en une membrane unique de coagulation, très visible mème à l'aril nu, comme lorsque l'on opère avec la cicutine type.

Ce pouvoir coagulant n'appartient pas aux produits goudronneux ' [ui accompagnent la base extraite des Arum. L'expérience suivante le prouve. Le produit mixte, impur, formé de cicutine et de goudrous, est traité par l'eau acidulée de $\mathrm{SO}^{4} \mathrm{H}^{2}$, épuisé à plusieurs reprises, puis lavé à l'eau jusqu'à disparition totale de l'acidité. Le goudron, débarrassé par ce traitement de toute trace d'alcaloïde, est impuissant ì déterminer dans l'albumine le moindre louche visible à l'ceil nu, ou la moindre précipitation de granules, visibles au microscope. D'ailleurs la solution acide de l'alcaloïde d'Arum détermine la même coagulation.

Dragindonfr estime que cette propriété de coaguler l'albumine 
ne peut être utilisée pour lil caractérisition de la conicine. Lil chuse: est exacte en ce qui concerne les recherches toxicologiques, où l'emploi d'une quantité extrêmenent minime de matière est de rigueur; mais pour le chimiste, cette réaction est d'une sensibilité parfaite, surtout si l'on recourt à l'examen microscopique.

Nous navons pas eu à notre disposition une quantité suffisante de chlorhydrate de notre alcaloüde, pour essayer son action sur la lumière polarisée. On sait que les chlorhydrates de conicine et de méthylconicine jouissent d'un pouvoir rotatoire.

Nous avoris en vain essayé d'obtenir, avec l'alcaloïde d'Arum, la coloration bleue verdatre, en présence de $1 \mathrm{Cl}$ à 1,2 de densité, donnée parfois comme caractéristique de la cicutine. Cette réaction d'ailleurs ne serait, d'après Dragkivorkf, nullement caractéristique de cet alcalö̈de; elle ne se produit que dans des circonstances mal déterminées, avec d'autant moins de netteté que le produit expérimenté est plus pur (on ne lobserve pas avec le résidu abandonné par le pétrole), et nous nous sommes assuré qu'on peut „orl bien ne pas l'obtenir avec la cicutine type.

Tous ces caractères correspondent à ceux de la conicine; nous avons d'ailleurs effectué comparativement les mênıes réactions avec de la conicine vraie; les réactions de la base des Arum se sont montrées identiques, quoique moins nettes par suite des impuretés qu'elle contenail, et dont nous n'avons pu la débarrasser complètement, étant donnée la faible quantité que nous avions pu isoler.

Cet alcaloïde ne semble pas d'ailleurs aroir de point spécial de localisation; on le retrouve aussi bien dans les tubercules que dans les tiges, les feuilles, les fruits et les graines.

Les caracteres ci-dessus indiqués suffisent à distinguer notre alcaloïde d'avec la nicotine.

Quant aux autres alcaloïdes volatils végétaux, bien définis lobé- 
liine, spartéine), ou encore à peine connus fprincipes alcalö̈diques volatils d'IHyosciamus nigpr (?), de Sturacenia purpurea, de Capsirum ammum], un seul possède l'odeur de souris si caractéristique de l'alcaloïde d'Arum et de la conicine, c'est celui que Felletar et Draraxnorfa ont indigué dans le piment. Mais l'alcaloüde de c'apsicum est encore si mal connu, qu'il serait prématuré de chercher à indiquer ses caractères différentiels, précis d'avec l'alcaloïde d'Arum (1).

La proportion si étonnamment minime de l'alcaloïde ci-dessus, dans les tissus des Arum, pourrait donner à supposer que nous n'avons réussi à extraire qu'un produit de dédoublement du véritable alcaloïde, qui existerait dans la plante en quantité plus notable. La faiblesse extrême du rendement pourrait être due, en partie, au traitement par la potasse. Nous nous promettons d'essayer, sur de nouvelles quantités de plantes fraîches, la méthode d'extraction par l'acide tartrique, appliquée couramment pour les alcaloïdes solides. Notre alcaloïde a d'ailleurs été recueilli dans le liquide distillé. Or la vapeur d'eau entraîne la conicine, mais la décompose en même temps, partiellement.

La teneur des Arum en alcaloïde n'est nullement comparable à celle de Conium maculatum en conicine. On sait, en effet, que la grande ciguë renferme de la conicine, à raison de 0,02 à 0,0 ö \% dans les tiges et feuilles a l'état frais, et 0,70\% dans les fruits mûrs (la proportion d'alcaloïde est encore plus forte dans les fruits recueillis avant maturité).

Nous verrons, plus loin, dans la relation des essais physiologiques que l'alcaloïde d'Arum est moins actif que la conicine de ciguë. Ce fait s'expliquerait si l'on admet que notre alcaloïde, bien que très analogue à la conicine vraie, en dillère quelque peu par sa formule chimique, et n'est qu'un dérivé de la conicinc', ou bien que l'alcaloïde par nous isolé est formé, comme l'alcaloïde

(1) Felletar. Pharmac. Zeitssch Rüssland. (t. II, p. 317). - DragendorfF. Manuel de toxicologie, trad. franç. (p. 382.) 
brut extrait de la ciguë, par le mélange de conicine vraie avec des dérivés; tels que la conhydrine et l'éthylconicine, dont l'action physiologique et la toxicité sont de même nature, mais beaucoup plus faibles que celles de la conicine.

Un alcaloüde a élé déjà signalé chez une Aroïdée : Arorus ralamus : la calamine de 'lirous.

Il est intéressant de comparer les propriétés de cet alcaloïde d'Acorus à celles de l'alcaloïde d'Arum.

La calamine est un corps cristallisé et facilement soluble dans l'alcool, le chloroforme, l'acétone, les acides étendus; insoluble dans l'eau et dans l'éther, elle ne réduit pas la liqueur de Fehling; elle jouit de propriétés basiques très nettes, renferme de l'azote, présente toutes les réactions connues des alcaloïdes; son chlorhydrate réduit facilement le chlorure de platine.

On peut l'obtenir par deux procédés :

10 L'extrait aqueux de la plante, en solution dans l'eau, est mélangé de tannin; le dépôt qui se forme est lavé sur un filtre, avec de l'eau acidulée par le même acide; ce dépòt, encore humide, est mélangé d'alcool, puis séché; le résidu sec, réduit en poudre, est traité par l'alcool absolu chaud. L'alcaloïde est obtenu à l'état de pureté par dîstillation de l'alcool;

$2^{\circ}$ La plante est soumise à l'ébullition à plusieurs reprises, dans de l'eau additionnée d'acide chlorhydrique, puis soumise à l'action d'une fortc presse; le liquide exprimé est filtré, puis neutralisé par du carbonate de soude, l'addition de tannin détermine un précipité; ce précipité, après lavage, est mélangé à de la chaux vive, séché, puis traité à plusieurs reprises par l'alcool chaud; après évaporation de ce dernier, on recueille l'alcaloïde faiblement coloré en jaune, imparfaitement cristallisé et encore impur.

Le rhizome d'Acorus donne environ 0,12\% d'alcaloïde.

On voit qu'il n'existe, à aucun point de vue, une analogie quelconque entre cette calamine et la base des Arum.

La présence dans un groupe végétal naturel, aussi éloigné des Ombellifères que celui des Aroïdées, d'un alcaloïde, sinon identique, au moins très voisin de la conicine ou de ses dérivés immé- 
diats (conhydrine, ethyl - et méthylconicine) est d'un réel intérêt au point de vue de la chimie végétale et de la biologie générale.

Il n'est pas sans intérêt de rappeler ici que, dans un groupe bien éloigné des Ombellifères, celui des Légumineuses-Papilionacées, on a rencontré un alcaloïde de même composition que la conicine (sans reparler de l'alcaloïde de Cupsicum).

Cette base, découverte dans les graines de Lupinus luteus par STENER, avait été identifiée par lui à la méthylconicine (Landw. Vers. Stat., t. XIV, 1872. - Arch. f. Pharm., t. I, $3^{\mathrm{e}}$ série, p. 40, 1872). Les recherches de Baunert ont montré qu'un des alcaloïdes de Lupinus luteus offre la même composition que la conicine, mais que, comme la paraconicine, préparée synthétiquement par ScmrF, il n'a en rien les propriétés physiologiques de la conicine vraie.

La lupinidine liquide de BAUMERT, son hydrate et la lupinidine solide sont donc, au point de vue chimique, de vraies conicines extraites des Légumineuses (Annal. d. Chem. . Phys., t. CCXXIV, p. 321, 1881).

Si, dans certains groupes végétaux, l'identité des principes chimiques révèle des affinités dues à une communauté d'origine phylogénique, dans certains autres groupes cette identité n'est que le résultat d'une convergence physiologique entre types fort éloignés.

ACIDE CYANBYDRIQUE

La présence de l'acide cyanhydrique, en proportions assez faibles d'ailleurs, dans les jeunes pousses d'Arum maculatum au printemps, a été indiquée en 1884 par JonIssex; la vérification de ce fait présente un vif intérêt au point de vue toxicologique : elle a donc sollicité d'une façon spéciale notre attention. D'ailleurs quelques Aroïdées exotiques élahoreraient, elles aussi, de l'acide cyanhydrique.

Les différentes espèces du genre Dieffenbachia, et en particulier D. Srgnime Scurrtr, une des espèces les plus fréquemment cultivées dans les serres, sont réputées très toxiques. 
En particulier, Dieffenbrehice Parlatorei, LANDEn et Avdré (Philodendron ENGr.), observí al l'ilat satuvage sur les bords du Rio Magdalena (Nouvelle-Gronado par E. André, exhalerait, lorsqu'on brise ses feuilles, une odeur forte d'acide prussique.

Cette odeur serait suffisanle. d'après certains auteurs, à catratériser les représentants du genre Dieffenbachia?

\section{Methode de recherches}

Pour rléceler la frésence de l'acide cyanhydrique ou de composís cyanridans les tissus des Arum, nous avons eu recours aux procédés suivants :

a) Ou distille une certaine quantité de plantes fraiches a vec sól $I^{2}$ étendu, on recueille ce qui distille. Le liquide distillé, soupronné de contenir ce produit, est saturé à peu près exactement par de la potasse; la lífueur est alors traité par quelques fouttes d'une solution d'un sel ferroso-ferrique (la solution aqueuse du sulfate ferreux oxydé du commerce convient tris bien). On filtre et on lave le précipité sur filtre à l'acide chlorhydrique faible; le bleu de Prusse doit rester sur le filtre, si le liquide essayé contient de l'acide cyanhydrique.

Nous avons contrôlé la sensibilité de cette méthode, sa limite inférieure est de $0 \mathrm{gr}$. 00 . de $\mathrm{CAzII}$.

b) Dans un liquide contenant une solution de CAzI étendu ia $\frac{1}{1000}$, on peut mettre à profit la réaction de Schönbein. Un papier imprégné de sulfate de cuivre à $\frac{1}{1000}$, additionné de quelques gouttes de teinture de gayac, récemment préparée, donne, en présence d'une solution d'acide cyanhydrique, une coloration bleue.

Une trace de $\mathrm{CAzH}$ gazeux, dans un espace clos, donne la méme réaction. Nous avons répété celle-ci avec le papier réactif, placé dans le liquide distillé comme il est dit ci-dessus, ou suspendu dans le courant de vapeur en distillation.

c) Un autre procédé, quelque peu différent, spécialement recommandé dans la recherche toxicologique de CAzII ou de ses dérivés, a été également employé.

On acidule les matières avec de l'acide tartrique sans excès, on distille au bain-marie, on fractionne les produits distillés dans de petits tubes, de façon à recueillir $2-3^{3}$ de liquide dans chaque tube, pour $100^{c^{3}}$ de matière.

CAzH, très volatil, est concentré dans les premières éprouvettes.

$1^{\circ} \mathrm{Au}$ distillat, on ajoute une solution de sulfate ferroso-ferrique + un léger excès liOH caustique; on agite, on ramène l'acidité par HCl. Coloration bieue, il se dépose du bleu de Prusse.

2* Au distillat, on ajoute AzII4IL'S, on évapore à chaud pcur chasser 
l'excès de ce réactif, on ajoute de 1 à 2 gouttes d'HCl +1 seule goutte de perchlorure de fer officinal. Coloration rouge de sulfocyanure de fer en présence de CAzH.

$3^{\circ}$ Le distillat est rectifié sur du borax, on acidule par l'acide nitrique, on y ajoute de l'azotate d'argent. Précipité de cyanure d'argent, s'il contient des traces de CAzII.

\section{RÉSULTATS}

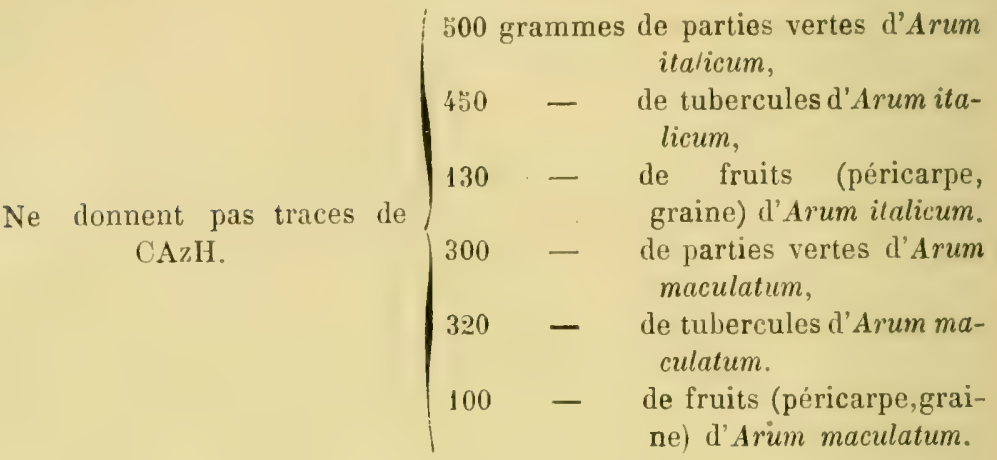

Les pieds de Dieffenbachia Seguine, cultivés en serre au jardin botanique de la Faculté, n'exhalent pas, quand on les froisse, la moindre odeur de $\mathrm{CAzH}$. La recherche de cet acide dans cette plante est restée, comme pour les Arum, infructueuse; mais il s'agit d'une variété panachée, et l'on pourrait supposer (hypothèse peu vraisemblable d'ailleurs) que la culture aurait si profondément modifié son chimisme, que CAzH, présent chez la plante sauvage, ne serait plus élaboré par ses variétés horticoles.

Les parties aériennes et souterraines d'Arisarum vulgare, les tubercules d'Amorphophallus Rivieri et de Caladium tuberosum sont également privés de cet acide.

L'absence de CAzII chez les Aroïdées semble donc, en dépit des assertions de quelques auteurs, un fait général. D’ailleurs les symptômes et les lésions de l'intoxication par les Arum el l'Arisarum ne concordent en rien avec ceux de l'intoxication par CAzII ou ses dérivés. 
Bien que ces considérations ne se raflachent qu’indirectement au sujet speicial qui nous ocempe, nous pouvons fitire remarefuer, qu'au point de vue de la biologie générale,il est d'un réel intérêt de vérifier la prósenes ou l'absence de CAzll dans lus divors régritaux, oủ il a été signalé par des auteurs récents. On sait, en effet, que Treub l'a récemment considéré comme un des premiars produits de l'assimilation dans les organes verts des plantes qui le contiennent. S’il est vrai que, chez les Amygdalies, les Pomacées, les Pamjum, CAzH existe en quantité assez nobable pour jouer ce rôle important, il semble plus difficile de le lui faire jouer chez les quelques aulres plantes, oì il n'a été signalé qu'en quantité très faible (Ribes aureum, Aquileyza v'ulgaris, I'on aquatira), au moment de la floraison, d'après Jorrssev. Quoi qu'il en soit, le's Aroïdées indigènes ne peuvent être considérées comme des plantes renfermant $\mathrm{CAzH}$ en quantité appréciable (supérieure à $1^{\mathrm{mmg}}$ par kilog).

Bibl. 1890. - Greshoff. Eerste Verlag van het anderzœk, naar de Plantenstoffen van Nederl. Indie. (Ann. du Jard. Bot. de Buitenzorg. Leyde.)

- 1895. - Treub (MI.). Sur la localisation, le transport et le rôle de l'acide eyanhydrique dans le Pangium edule (1bid.).

\section{B. - Arisarum vulgare.}

\section{PRINCIPES ACTIFS}

(Mème méthode de recherches que pour Arum.)

$1^{\circ}$ Parties vertes. (Feuilles et inflorescences.) $300 \mathrm{gr}$. donnent:

$\mathrm{CA} 7 \mathrm{H}$

Base volatile, abondante par / odeur de conicine, distillation avec $\mathrm{KO}$ H et extrac- $\{$ fumées en présence de $\mathrm{ICl}$, tion à l'éther.

I précipité jaune brun par KI ioduré.

Saponine dépòt par refroidissement, dans l'extrait par l'alcool bouillant fait mousser l'eau, après interversion, réduit la liqueur de FEHLING. 
$2^{\circ}$ Rhizômes (conservés it sec depuis 8 jours, d'oil déperdition possible des principes volatils). $200 \mathrm{gr}$, donnent :

$\mathrm{CAzH}$ Base volatile, en très faible
quantité. $\left\{\begin{array}{l}\text { odeur de conicine, } \\ \text { les réactions caractéristiques sont peu } \\ \text { nettes. }\end{array}\right.$

Saponine

(dépôt assez abondant dans l'extrait par l'alcool bouillant par refroidissement, fait mousser l'eau,

après interversion, réduit la liqueur de FEHLING.

Pendant la période de floraison et de fructification, Arisarum z'ulgare contient donc, comme les Arum: $1^{\circ}$ de la saponine (qui, même à cette époque de végétation, est plus abondante dans les parties aériennes); $2^{\circ}$ une base, vraisemblablement identique à celle des Arum, dont toutes les analogies sont avec la conicine (à cette époque, cette base est surtout abondante dans les parties aériennes; pendant la période de repos de la plante, elle doit être en plus notable proportion dans les parties souterraines); $3^{\circ}$ on ne peut constater trace d'acide cyanhydrique.

\section{Conclusions.}

Nos essais nous permettent d'arriver, relativement aux principes actifs des Arum et de quelques autres Aroïdées, aux conclusions suivantes :

a) Conformément à l'opinion de Enz, Spica et WaAge, tous les organes de ces plantes sont plus ou moins riches en saponine, ou plus exactement en un glucoside du groupe des saponines. La proportion de ce corps, dans les divers organes, varie selon les différentes époques de végétation, sa proportion maxima (tubercules au repos) ne dépasse pas 1 pour 1.000 du poids de la plante fraîche.

b) Le principe âcre, que personne n'était parvenu ni à caractériser, ni mème à extraire, est un alcaloïde liquide, nettement caractérisé 
comme voisin, sinon identique, ì la conicine ou aux bases dérivén de celle-ci; cet alcaloïde se retrouve dans tous les organes, c'est dans les fruits que sat proportion semble itre la plus forte. Sat proportion maxima semble être de $0,0030,00$ de plante fratiche.

c) L'acide cyanhydrique est impossible à déceler, en quantité appréciable, dans les Aroïdées par nous étudiées.

BInL. 184 - Dietionnaire des drogues simples et composées, I, p. 333. (Cité dans Mérat et DeLEns.)

1858. - Enz. Présence de la Saponine dans l'Arum maculatum. IVittstein's. Vierteljahr. t. VIII, p. 27. - Analys. Bull. Soc. chim. de Paris. - Répertoire de chimie appliquée, t. I, $1^{\text {re }}$ année, p. 17'.

1882. - Husemann (A. et Theod.) et IHllger (A.), Die Pplanzensto/fe, I, 414, Berlin.

1884. - Jorissex. Bildung von Cyanwasserstoffesaüre (Journ. pharm. Anvers, p. 362. - Journ. de pharm. et de chimie, sér. 5艹, t. II, p. 286).

1885. - Jorissen (Pharm. Zeitung f. Rüissland, n³ 34 p. 321. Archiv. f. pharmacie (3) XXIII, p. 513).

1883. - SpICA (G.) (Annal. di chimic. med. farmac., p. 94).

1885. - Spica (G.) et Biscaro (G.). (Gaz. chemic ital., t. XV, p. 238.)

1886. - Thows (H.) (Pharmac. Centralhalle f. Deutschl. no 49, p. 614. - Rundsch. f. die Interes. Pharmac. chem. n०26, p. 517, n०50, p.997.)

1892. - WAAGE (TH.) Verbreitung der saponinartigen Stoffe im Pflanzenreiche (Pharm. Centralh. 657, 673, 685, $696,712$.

1893. - Id. (Apothet. Zeitung, 7\%.) 


\section{CHAPITRE III}

\section{ÉTUDE EXPÉRIMENTALE DES PROPRIÉTÉS TOXIQUES DES ARUM.}

Les propriétés toxiques des Arum sont proclamées par tous les auteurs, mais aucune recherche physiologique, séricuse et méthodique, n'a cependant été faite, jusqu'à ce jour, relativement à la toxicité des Aroïdées indigènes.

Les vieux auteurs se contentent d'établir un rapprochement entre les symptomes de l'intoxication par les Arum et ceux du choléra. Ces plantes sont généralement regardées comme des poisons violents, d'autant plus dangereux qu'elles sont presque insipides, lorsque l'on commence à mâcher leurs organes; mais bientôt une saveur âcre et brûlante se développe dans la bouche, qui semble piquée par des milliers d'aiguilles : gonflement intense de la langue et du pharynx, stomatite, edème de l'arrière-bouche, si considérable qu'il rend toute déglutition impossible, sensation de brùlure dans le pharynx et à l'épigastre, vives douleurs stomacales, vomissements, coliques, superpurgation, convulsions, petitesse du pouls, refroidissement progressif, crampes, tel serait le cortège des symptômes de l'intoxication par les Arum.

Toutes les parties de ces plantes produiraient des désordres analogues. Les feuilles seraient plus actives que les racines (MÉrat et Delexs). Orfila dil avoir fait périr des chiens, en leur faisant ingérer le rhizome frais, la mort survenait de 20 à 36 heures après l'ingestion, par suite de l'irritation gastro-intestinale. Le suc "extrait du tubercule serait moins âcre que celui-ci, un peu acide, et le précipité qu'il dépose ne le serait pas du tout "(DuLovg). (Ce précipité est d'ailleurs formé en majeure partie d'amidon.) On trouvera plus 
loin le résumé des quelques observalions précises que nous possédons, relativement a des cas d'intoxication loujours graves, sonvent mortels, à la suile de l'ingestion, par des enfants, des feuilles et surtout des fruits, des jeunes pousses vertes, et plus souvent des rhizomes, par les animaux domestiques.

Nous chercherions en vain, dans les écrits des nombreux auteurs qui ont parlé d'une manière plus ou moins superficielle des propriétés toxiques des Arum, des données précises, capables de nous éclairer sur la toxicité réelle, sur le mode d'action physiologique et sur la nature des principes actifs de ces plantes. Pour combler celte lacune regrettable, tant au point de vue de nos connaissances de toxicologie pure sur les plantes incligènes, que des applications hygiéniques et médico-légales, nous avons tenté une assez longue série de recherches dont nous allons donner un résumé succinct.

Dans notre étude, l'important n'est pas d'exposer une sírie plus ou moins longue d'expériences qui se reproduisent sensiblement identiques. Il nous suffira de donner le détail de certaines d'entre elles, et de dégager de l'ensemble les faits les plus saillants, capables de fixer nosidées sur la réaction de l'organisme animal, en présence des substances toxiques étudiées.

\section{I. - Action sur les téguanents.}

Les différents organes des Arum, ou le suc qui en découle, sont réputés, d'une façon générale, àcres, irritants, caustiques et même vésicants.

Les fragments de rhizome, les feuilles pilées et appliquées immédiatement sur la peau, posséderaient une action révulsive analogue à celle de la farine de moutarde (Massé). Ce serait à la fois des rubéfiants et des vésicants; “ j’ai souvent employé ce vésicatoire » avec succès », dit Cizıs. Le suc d'Arum serait un caustique, capable de détruire les végétalionseł mème les polypes nasaux (Matrole).

Voyons ce que ces assertions ont de justifié. Le contact prolongé du suc exprimé des organes verts détermine, sur la face dorsale des mains, une démangeaison quelque peu désagréable, mais fugace. 
Lorsque l'on manie les tubercules d'Arum, en les débitant en tranches minces, le suc qui en découle occasionne, à la face dorsale des mains et à la partie antérieure desavant-bras, partout oùla peau est fine, des démangeaisons désagréables, que provoque également le contact de lamacération de ces tubercules dans l'eau. Ces démangeaisons se dissipent d'ailleurs rapidement, et bien qu'ayant manié, souvent pendant une heure et plus, des fragments frais ou macérés de rhizome, jamais nous n'avons constaté d'action ni vésicante ni même rubéfiante.

Nous avons alors essayé les prétendues propriétés révulsives des Arum sur divers malades, auxquels une révulsion énergique des téguments était supposée devoir être utile. Voici quelques observations à cet égard.

Exp. I. - Femme tuberculeuse, peau fine.

22 mai. - Application d'une rondelle de rhizome d'Arum dans la fosse sous-épineuse. Très légers picotements momentanés, disparaissant rapidement, effets vésicants et même rubéfiants nuls.

23 mai. - L'expérience est recommencée; même résultat négatif.

L'application d'une simple rondelle de rhizome peut être considérée comme dépourvue de tout pouvoir révulsif.

Exp. II. - Femme tuberculeuse, peau assez fine.

30 mai. - Friction énergique de la région scapulaire avec une tranche de rhizome, puis application de cette tranche à demeure. Cuisson imméliate, quelques démangeaisons persistant jusqu'au lendemain.

1 er juin. - L'expérience est répétée dans les mêmes conditions ; même résultat.

Exp.III. - 31 mai. - Autre malade tuberculeuse, peau très fine. Sur une étendue de 12 centimètres environ, à la région sous-claviculaire, frictions énergiques avec un rhizome cassé en deux. Légers picotements immédiats; pansement à la vaseline; quelques démangeaisons persistent jusqu'au lendemain.

1 er juin. - L'expérience est répétée le lendemain; même résultat.

Exp. IV. - Autre malade tuberculeuse, expérience entièrement comparable à la précédente.

Exp. V. - 31 mai. - Femme atteinte de bronchite suspecte, peau très 
fine, un cataplasme de rhizome frais est appliqué à la région dorsale, légers picotements; après une heure d'application, le cataplasme est enlevé et la région frictionnée, léger prurit dans le courant de la journée.

fer juin. - L'expérience est répétée avec le mème résultat.

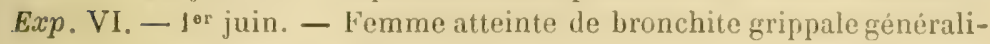
sée,peau très fine. Application à la région dorsale d'un cataplasme de feuilles fraiches pilées; après 10 heures d'application, ni picotements, ni la moindre rubéfaction.

2 juin. - Mème expérience, après frictions préalables assez énergiques de la peau, même résultat.

Exp. VII. - 1 cr juin. - Autre malade tuberculeuse, peau fine, expérience absolument comparable à la précédente.

Exp. VIII. - 1 er juin. - Femme atteinte de bronchite chronique, peau fine, frictions énergiques de la région dorsale avec des feuilles fraiches contusées, pendant 15 minutes; résultat nul.

De ces essais il résulte, qu'en pleine période de végétation, les Arum, loin de posseder un pouvoir vésicant, sontimpuissants à déterminer la simple rubéfaction d'une peau fine. Tout se borne à un prurit, plutòt léger, peu persistant. Le rhizome paraît, à ce point de vue, un peu moins inactif que les parties vertes. Le suc filtré, par suite privé de raphides, peut aussi déterminer quelques picotements; ceux-ci sont donc dus beaucoup plutôt au principe àcre de la plante qu'à l'irritation mécanique, déterminée par l'implantation des raphides dans les téguments.

Le dépôt (recueilli par filtration) d'un suc riche en raphides peut être frotté sur lí peau sans déterminer d'irritation notable; les raphides peuvent donc tout au plus faciliter, par les minuscules érosions qu’ils déterminent, l’arrivée du principe àcre au contact des couches sensibles de l'épiderme.

\section{II. - Action sur les nuqueuses.}

Si l'on porte à la bouche un rhizome frais d'Arum, et qu'on l'entame avec les dents, on ressent une brùlure immédiate, qui fait vite làcher prise, et peut persister, tout en décroissant peu à peu d'intensite, pendant plusieurs heures. La mastication des parties verte et des fruits détermine également cette sensation d'àcreté et de 
brulure; mais nous n'arons janais pu constater sur nous-mème, ni sur diverses personnes qui, par curiosite, avaient mordu ou mastiqué des tissus d'Arum, la moindre tuméfaction des muqueuses ayant subi le contact de ces lissus. Il en a été de même, au cours des nombreuses expériences où nous faisions ingérer à des animaux du suc d'Arum, même lorsque ce sucétait déposé goutte à goutte dans le pharynx; jamais ces animaux n'ont présenté une tuméfaction bien appréciable du pharynx, de l'épiglotle ou du voile du palais.

L'introduction accidentelle de quelques gouttes de suc, dans les voies respiratoires, détermine de violents efforts de toux, mais il n'y a rien là de bien spécial au sue d'Arum.

Ce dernier vient-il, en tombant dans l'arrière-bouche, à pénétrer sous l'influence du courant d'expiration, dans les fosses nasales, il ne provoque pas de bien énergiquesréflexes d'expulsion els'écoule peu à peu, grâce à de petits mouvements dès narines, sans provoquer grande réaction.

L'action irritante sur les muqueuses du suc d'Arum a donc été tout aussi singulièrement exagérée que celle qu'il exerce sur les téguments.

III. -- L'intoxication expérinentale par les Arum ET LEURS PRINCIPES TOXIQUES.

A. - Essais dintoxication par voie digestive.

\section{$1^{\circ}$ Organes en nature.}

Il est presque impossible de faire ingérer aux animaux de laboratoire des quantités un peu notables d'organes frais d'A'um en nature. Les herbivores refusent d'y toucher spontanément, et préfèrent le jeùne complet à l'ingestion de feuilles ou de rhizomes; ils ne touchent également pas aux rhizomes desséchés. Le chien n'avale de force qu'une faible quantilé d'organes frais, réduits en pulpe; leur sareur âcre, brûlante, détermine de violents mouvements d'expulsion qui s'opposent totalement à l'expérience. On ne 
peut songer it fitre passer, mème chez le chien, de la pulpe d'organes par la sonde œsophagienne.

Par là mème, le mode d'expérimentation est forcenent rustreint. Aucune expérience d'ingestion d'organes frais, fùt-ce même de fruits de petite dimension, ne réussit chez le cobaye et le: litpin, il y a toujours rejel.

La pulpe fraîche de feuilles d'Arum, mélangée aux aliments, rebute aussitot le chien; l'animal qui y a touché lémoigne, par sa salivation, les mouvements d'expulsion auxquels il se livre, de la sensation de brùlure qu'il ressent. La mème pulpe, desséchée, puis humectée à nouveau, est ingérée sans difficulté, avec les aliments, en quantité notable (100 grammes), sans déterminer d'effets fàcheux; tout au plus l'animal témoigne-t-il, pendant et après l'ingestion, d'une sensation de picotements sur la muqueuse buccale. La mème pulpe, après ébullition, peut être également ingérée, sans déterminer d'autres effets que la précédente. Les mêmes expériences, tentées avec le rhizome, donnent des résultats identiques.

Le tubercule, après ébullition, ne présente plus trace de saveur àcre, malgré les raphides inaltérés qu il contient, il peut alors être mastiqué et avalé sans inconvénient. En le goùtant, nous n’avons éprouvé aucune sensation appréciable de brùlure ni de picotement. Il est alors accepté volontiers par le chien; sa consistance quelque peu gélatineuse, son odeur presque nulle, en font un aliment insipide, mais dont l'ingestion n'a rien de désagréable.

Nous avons pu faire ingérer au chien jusqu'à dix tubercules, après ébullition, chacun d'un poids moyen de כ̃0 grammes. Aucun malaise appréciable.

Avec de la patience et de l'adresse, il est possible, en enrobant dans du sucre ou de la viande des fruits d'Armm (péricarpe et graines), d'en faire ingérer au chien un certain nombre; dix ou douze fruits peuvent ainsi être administrés, mais la pression qu'ils subissent, lors de la déglutition, fait exsuder leur suc, l'àcreté de celuici détermine des efforts d'expulsion, qui empèchent de continuer l'expérience. 
La pulpe fraîche de fruits, mélangée aux aliments, est également refusée; desséchée, puis humectée, après ébullition, elle peut être ingérée, comme celle de feuilles et de tubercules, sans déterminer d'autre phénomène que celle-ci.

Les fruits qui ont subi une dessiccation assez avancée ¿̀ l'air, peuvent être administrés à la manière de pilules, et en quantité assez considérable.

Exp. IX. - Chien neuf. Poids 13 k. 500.

$10 \mathrm{~h}$. - Ingère successivement 50 de ces fruits. Quelques efforts d'expulsion, des nausées sans effet témoignent d'une certaine irritation des premières voies digestives.

2 h. - Ingestion de 50 nouveaux fruits, mêmes phénomènes. Observé pendant les deux jours suivants, le chien ne témoigne d'aucun malaise, mais mange avec moins d'appétit et présente une constipation opiniâtre. Lorsque le cours des matières fécales se rétablit, il expulse des fèces, extrèmement dures, où les graines ingérées avec les fruits sont réunies par une sorte de masse gommeuse. On retrouve, en les délayant, des débris de péricarpe, encore riches en raphides.

Exp. X. - Dans les mêmes conditions, le même chien et 2 autres animaux ingèrent chacun, pendant 2 jours de suite, 100 fruits desséchés. Tous trois présentent, dès le lendemain, une constipation opiniâtre qui ne cesse qu'au hout de 4 jours. Ces expériences sont donc très concordantes.

Loin donc de déterminer, par la présence de ces raphides, une irritation intestinale traduite par de la diarrhée, les fruits d'Anm, ingérés en nombre assez considérable, déterminent au contraire, une constipation fort nette. La cause de celle-ci est toute mécanique. Comme tous les organes de la plante, le péricarpe d'Arum est riche en gomme, lorsqu'il récupère, au contact des sucs digestifs, l'eau dont l'arait privé la dessiccation; la gomme qu'il renferme agglomère et moule les graines en masses dures, cimentées par les matières fécales, et qui opposent ainsi un obstacle mécanique au cours de ces dernières. Il est à noter que d'autres fruits, ègalement réputés toxiques, peuvent déterminer aussi des phénomènes d'embarras gastro-intestinal par le même mécanisme. Un exemple fort net est fourni par les fruits de Gui (Viscum album), dont la 
richusse en matières gommeuses ast infiniment superieure it celle des fruils d'Arum. Il y a lì une cause d'erreur (qui semble avoir jusqu'ici échappé à l'observation) dans l'appréeciation des disordres gastro-intestinatux, qure certains fruils, riche's en gomme, sont censés produire, lorsqu'on les ingère avec leurs graines.

\section{$2^{\circ}$ Suc extrait des organes.}

Le procédé le plus sûr pour expérimenter l'action physiologique des Arum administrés par les roies digestives, consiste a se servir du sue que renferment leurs organes. Les tissus frais, hachés, sont soumis à la compression énergique d'une presse, il s'en écoule un suc gommeux, riche en raphides, de saveur ìcre, et qui contient en dissolution la majeure partie des principes actifs de la plante. L'analyse chimique permet d'y retrouver la saponine et l'alcaloïde.

Dans tous les essais d'intoxication par ingestion, nous avons toujours fait pénétrer le toxique par la sonde œsophagienne. Le poison mêlé aux aliments leur communique une saveur ou une odeur qui rebutent l'animal; même en cas d'ingestion volontaire par ce dernier, une portion plus ou moins notable peut toujours ne pas ètre ingérée. La sonde œsophagienne est d'ailleurs la seule méthode à employer, pour faire ingérer d'une façon sùre aux herbivores des solutions ou des substances semi-liquides.

L'animal est tenu par un aide la tête en extension, les mâchoires sont écartées par un bâillon, proportionné à la grandeur de la gueule de l'animal, un trou central livre passage à la sonde. La quantité de liquide que l'on veut faire ingérer est versée dans une ampoule, dont la portion inférieure rétrécie s'adapte à frottement au pavillon de la sonde. La partie supérieure de l'ampoule recoit un bouchon de caoutchouc, avec tube coudé, relié à une poire de Richardson. La pression qu'exerce dans l'ampoule, sur le liquide à injecter, l'air foulé par la poire, permet d'instiller goutte à goutte dans l'estomac plusieurs centimètres cubes d'une solution, fùt-elle même gommeuse et fortement visqueuse.

Au cas où, par le vomissement (si fréquent et si facile à déterminer chez le chien), l'animal en expérience rejette, avec des mucosités, une portion plus ou moins notable du liquide ingéré, il est facile, en placant à l'avance l'animal sur un large plateau émaillé de recueillir le liquide expulsé, et de le réintroduire par la sonde, lorsqu'au bout de quelques instants la tolérance gastrique est rétablie.

Il est de la plus grande importance, mème chez les animaux dont le 
sondage cesophagien est facile (chien, cobaye), de bien s'assurer que la sonde est assez enfoncée dans l'œsophage, pour qu'aucune parcelle du liquide ingéré ne reflue dans le pharynx, d'où il pourrait pénétrer dans l'appareil respiratoire. Sa présence dans les bronches ou les alvéoles pulmonaires, surtout s'il contient, comme le suc d'Arum, des particules solides, capables de déterminer des lésions mécaniques, pourrait déterminer des lésions de broncho-pneumonie, le plus souvent infectieuse. Si le toxique n'agit qu'àlongue échéance, la mort du sujet en expérience, survenant 24 ou 48 heures après l'expérience, pourrait être mise sur le compte, non de l'intoxication mais d'une pneumonie intercurrente.

\section{a) Expériences avec le suc brut.}

Parties vertes. - Les parties vertes (feuilles et inflorescence) des Arum, à l'époque de leur pleine végétation, donnent, après expression à la presse, environ $280 / 0$ d'un suc, dont la densité est très faible (indice de la faible proportion des matières tenues en dissolution dans les cellules). Chaque centimètre cube de ce suc correspond, à peu près, à 3 grammes de parties vertes fraîches. La quantité de suc fournie par le péricarpe des fruits à maturité est à peu près la même; cette quantité est plus faible pour le suc cxtrait des rhizomes. Pour nous en tenir à un chiffre général, très suffisant comme approximation, nous pourrons dire que chaque centimètre

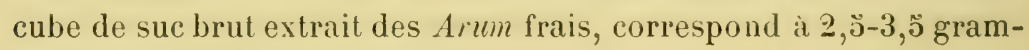
mes de l'organe qui l'a fourni.

\section{Cobaye.}

Exp. XI. - Cobaye mâle, neuf. Poids $800 \mathrm{gr}$.

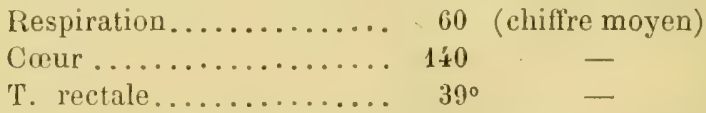

$1 \mathrm{~h}$. - Ingestion par la sonde cesophagienne, enfoncée dans l'estomac, de $10^{\text {e3 }}$ de suc. Rien à noter jusqu'à 1 h. 3u. - Légère accélération de la respiration.

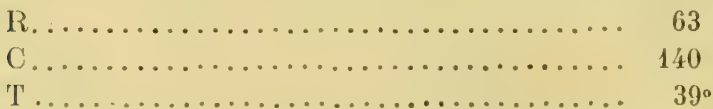

$2 \mathrm{~h}$. - La respiration est revenue à la normale. 
Le lendemain, l'animal est en santé parfaite, pas trace de diarrhée, appétit. Pendant toute la durée de l'expérience, grande vivacité, réaction facile à toutes les excitations.

A la dose de $10^{\mathrm{e} 3}$, le suc peut ètre considéré conme totilrment inactif.

Exp. XII. - Cobaye, femelle, neuf. Poids 623 gx".

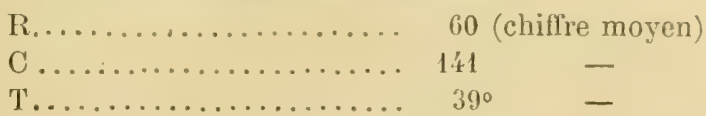

$4 \mathrm{~h} .-17^{\mathrm{c}^{3}}$ de suc dans l'estomac, aucune modification notable dans le rythme respiratoire et cardiaque ni dans la température jusqu'à $6 \mathrm{~h}$.

Le lendemain, pas trace de diarrhée, appétit; n'a cessé de présenter une grande vivacité et une réaction facile aux excitations.

A la dose de $17^{\circ 3}$, le suc est inactif.

Exp. XIII. - Cobaye, femelle, neuf. Poids $526 \mathrm{gr}$.

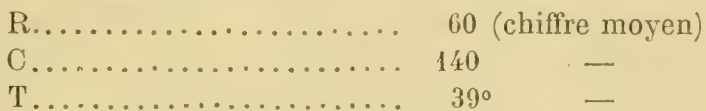

4h. 20. $-20^{\mathrm{c} 3}$ de suc dans l'estomac (sonde enfoncée profondément, son bec doit affleurer près du pylore). Injection un peu douloureuse.

4 h. 30 .

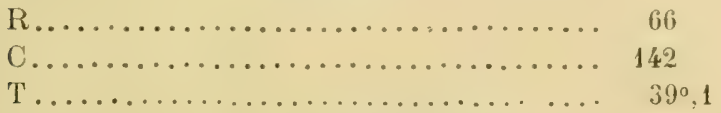

Un peu de dyspnée, de frissons, d'inertie.

6 h. - Retour à l'état normal.

Exp. XIV. - Même cobaye. Le lendemain, parait entièrement rétabli. Des fèces abondantes, semi-liquides, témoignent seules d'une irritation intestinale.

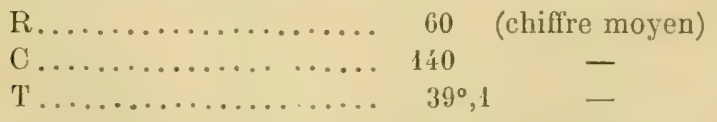

4 h. $-10^{\mathrm{c} 3}$ de suc dans l'estomac (sonde enfoncée profondément comme dans l'expérience ci-dessus). Sondage douloureux (indice de l'irritation des muqueuses œsophagienne et surtout stomacale); violents efforts dexpulsion. 
4 h. 10. - L'animal tombe sur le còté, puis se relève; apathic, sensibilité générale diminuée, subparésie du train postérieur, gémissements continus.

4 h. 13. - Agitation.

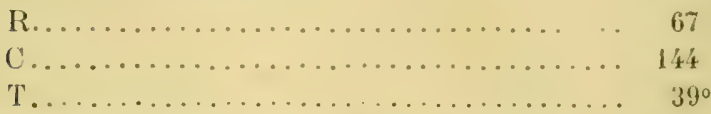

Tremblements. A cette période d'agitation, succède une période de calme et d'inertie.

4 h. 30. - Nouvelle chute sur le côté, iremblements.

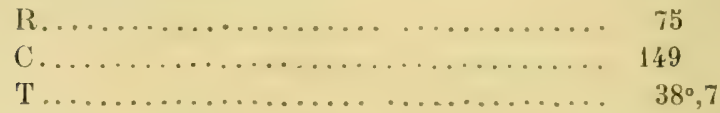

5 h.

R. ralentie.................. 56

C.......................... 120

T......................... 38

Sensibilité générale presque totalement disparue, le coma commence à apparaître.

s h. 15. - Coma complet, intermittences cardiaques et respiratoires, cœur fibrillaire, sensibilité générale et motricité disparues.

5 h. 30. - Sensibilité cornéenne presque nulle; arrêt de la respiration; le cour bat encore, mais à peine perceptible; petites convulsions partielles, convulsions finales. Mort.

6 h. 30. - Autopsie. - OEsophage : légère congestion, mucus abondant.

Estomac. - Quelque peu dilaté pardes gaz, une certaine quantité d'aliments mélangés de suc (raphides au microscope); fines arborisations vasculaires sur les deux faces, pas de congestion accusée; à la face interne, épaisse couche de mucus; muqueuse un peu friable.

Intestin. - Tout l'intestin grêle, surtout le duodénum, distendu par une sérosité sanguinolente; par places, mucus abon. dant, adhérent à la muqueuse; à l'examen microscopique : leucocytes, quelques hématies, cellules épithéliales, nombreux raphides, dans la sérosité. Le suc n'a pas pénétré dans le gros intestin, gonflé d'aliments, entièrement normal.

Rein.-Piqueté violet à la surface, larges plaques de congestion; sur la coupe tangentielle de la région corticale piqueté violet (congestion glomérulaire); injection assez vive de 
la zone des pyramides; la coupe longitudinale, passant par le hile, laisse écouler un sang noir assez abondant.

Vessie. - Très distendue.

Organes génitaux internes. - Congestionnés.

Foie. - Congestion légère; un peu friable; di la coupe, gouttelettes de sang noir.

Systeme veineux abdominal. - Rempli de sang fluide noir.

Poumons. - Franchement asphyxiques, larges sullusions sanguines, sous forme de marbrures noires à la surface, large zone lardacée; a la coupe : spume abondante, sang noir, le tissu crie sous le scalpel. Les deux poumons présentent une véritable hépatisation rouge, surtout à la base.

Caur. - C. gauche vide, ventricule en systole; cœur droit en diastole, gorgé de sang noir et liquide, ainsi que l'artère pulmonaire, les veines caves et leurs branches d'origine.

Lésions histologiques du tube digestif. - Les lésions du tube digestif (fixé aussitòt par le sublimé acétique) sont:

Estomac. - Mince couche pseudo-membraneuse de cellules épithéliales desquamées, avec leucocytes, le tout englobé par un mucus assez abondant; par places, la muqueuse est dépouillée de son épithélium cylindrique, cette chute épithéliale est localisée aux plis de la muqueuse. Pas de lésions appréciables des glandes. L'absence de toute prolifération cellulaire s'explique par la rapidité de l'intoxication. Un peu de congestion vasculaire au niveau des culs-de-sacs glandulaires. Le tissu conjonctif inter-glandulaire est assez fortement infiltré de leucocytes; l'infiltration, très marquée au voisinage de la surface, diminue progressivement vers la profondeur, où toute trace de diapédèse cesse d'être appréciable. Les autres couches de l'estomac sont indemnes de toute lésion.

Intestin grêle. - Sur presque toute sa longueur, l'épithélium est desquamé, ses cellules détachées sont fragmentées, leurs noyaux mis à nu, une grande quantité de leucocytes et d'hématies sont plongées, avec les débris épithéliaux, dans une couche de mucus. Les dépressions entre les villosités sont, par endroits, remplies de mucus avec leucocytes et hématies. Congestion sanguine, infiltration leucocytique intense et diffuse dans le tissu conjonctif des villosités. Aucune lésion appréciable des glandes ni des autres tuniques intestinales.

\section{Lapin.}

Il est superflu de donner la relation détaillée des essais d'intoxication par le suc frais brut, des parties vertes d'Arum, sur le lapin. Leurs résultats sont trop comparables à ceux obtenus sur le cobaye.

Un lapin adulte, de poids moyen (3 kilos), supporte impunément 20 à $25^{\mathrm{c}^{3}}$ du suc ci-dessus par voie stomacale; 30 à $33^{\mathrm{c} 3}$ 
determinent généralement une irritation gastro-intestinale, traduile par un ramollissement des malièresfócales, dans les 24 heures qui suivent l'ingestion. L'animal, abandonné à lui-même avec régime alimentaire sec, se rétablit, mais il succombe à une nouvelle ingestion stomacale d'une dose plus faible de suc 10 à $\left.16^{\mathrm{c} 3}\right)$. Pour les symptomes el les lésions déterminés par culte intoxication, nous ne pouvons que renvoyer à ce qui a été dit à propos des expériences sur le cobaye.

rhien.

Nous serons très bref dans la relation des expériences entreprises avec le suc brut des parties vertes, sur le chien, en raison du peu de résultats qu'elles fournissent.

Exp. XV. - Chien neuf. Poids 10 kilos. Reçoit dans l'estomac $50^{c 3}$ de suc. Légers efforts nauséeux, non suivis de vomissements. Rien de particulier à noter ; 24 heures après, état général excellent, appétit, fèces normales.

Exp. XVI. - Chienne neuve. Poids $8 \mathrm{k} .300 .75^{\mathrm{c} 3}$ de suc dans l'estomac; une portion est rejetée par le vomissement avec mucus abondant, aussitôt recueillie et réinjectée, quelques nausées sans résultat. Aucun symptôme d'intoxication ni d'irritation gastro-intestinale. Le lendemain fèces normales.

Exp. XVII. - 2 h. 43. - Chien neuf. Poids 11 k. 200. Dans l'estomac, $100^{c^{3}}$ de suc, en 2 fois, à $1 / 4$ d'heure d'intervalle, légers eftorts de nausées, sans résultat.

3 h. - Agitation, gémissements, nausées, vomissements de mucosités (raphides au microscope), respiration un peu haletante, cœur et température normaux.

Rien à noter jusqu'à $5 \mathrm{~h}$.

Le lendemain diarrhée (raphides au microscope), inappétence pendant 24 heures, puis rétablissement parfait.

Exp. XVIII. - Même chien. 2 jours après. $200 \mathrm{c} 3$ dans l'estomac en 4 fois. Mêmes sympòmes que dans l'expérience ci-dessus, la diarrhée n'est pas sensiblement plus avancée; le lerdemain, le chien se rétablit dans les 24 heures.

Exp. XIX. - Mème chien ayant déjà subi ₹ injections, l'une de 100 c3, l'autre de $200^{\mathrm{c} 3}$, reçoit, pendant 2 jours 50 c3, ', et pen-

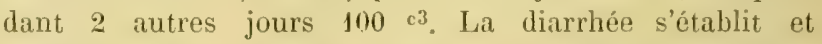
persiste pendant toute la durée de l'expérience; elle disparaît 48 heures après la dernière injection. L'animal ne témoigne que par cette diarrhée, par linappétence, et une perte de poids (7 k. 500 à la fin de l'expérience), les 
troubles gastro-intestinaux qu'il a éprouvés. Pour juger de l'état de ses organes digestifs, il est mis à mort par section du bulbe. L'autopsie ne révèle qu'une congestion, accusée par places seulement, des muqueuses gastrique et duodénale.

Exp. XX. - Expérience identique, répétée sur un autre chien neuf, du

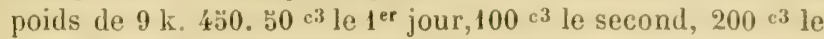
$3^{\circ}$. Mis a mort le $4^{0}$ jour, lorsque la diarrhée est établie.

Autopsie. - Congestion du duodénum; arborisation vasculaire, assez marquée par places, sur la face externe de l'estomac; couche épaisse de mucus sur toute la surface interne de l'estomac et de l'intestin grèle; ce dernier renferme un chyme jaunâtre, peu abondant (l'animal se nourrit peu), délayé par un liquide séreux, où le microscope révéle la présence de nombreux raphides, de cellules épithéliales entières, et fragmentées, dęlleucocytes et de rares hématies. L'examen histologique de la muqueuse n'a pas été pratiqué.

Tubercules. - Fruits. - Il serait sans intérêt d'insister sur les essais, tentés avec le suc frais non filtré des tubercules et des fruits mùrs, le seul point à mentionner est l'activité moindre du suc des tubercules; ce fait est en contradiction avec les assertions des auteurs, mais il est bon de noter que les tubercules, par nous employés; appartenaient à des plantes en pleine période de végétation, et, d'après ce que nous pouvons prévoir de la migration des principes actifs, il se pourrait que les tubercules possèdent une activité plus grande, pendant la période de repos.

L'activité du suc extrait des fruits (péricarpe seul) est très comparable à celle du suc des parties vertes.

b) Expériences avec le suc fermenté à l'air libre (non filtré.)

Pour les essais avec le suc d'Arum fermenté, desséché, filtré ou stérilise, nous nous sommes uniquement adressé au suc extrait des parties vertes. Le suc des rhizomes et des fruits, plus difficile à se procurer en quantité suffisante, n'était pas, d'après ce qui vient d'ètre dit, susceptible de fournir des résultals expérimentaux plus dignes d'intérêt.

Parties vertes. - Le suc ayant subi, à l'air libre, une fermentation de huit jours, présente une odeur sui generis; additionné de potasse à chaud, il ne dégage plus l'odeur de souris, caractéris- 
lique de l'alcaloüde; la saponine y est décelée par ce fait, que le suc mousse abondamment par agitation, ne posside qu'un pouvoir réducteur très faible, et que ce pouvoir devient notable après interversion (hydrolyse de la saponine). La saponine n'a donc pas subi d'action hydrolysante, de la part des diastases microbiennes. Inutile d'ajouter que ce suc est aussi riche en raphides que le sue frais.

Ce suc fermenté est d'une remarquable inactivité, lorsqu'il est ingéré par roie stomacale. 100 centimètres cubes, administrés en moins d'une heure, à un cobaye de poids moyen ne déterminent aucun ș̣mptôme d'intoxication. En présence de ce résultat, indiqué par plusieurs expériences, il est sans intérêt d'expérimenter, arec le suc fermenté, sur des animaux moins impressionnables : lapin, chien.

\section{c) Expériences avec le suc desséché (non filtré).}

Le suc frais, desséché à l'étuve ì s̃ $0^{\circ}$, est privé par évaporation. de l'alcaloïcie. La saponine et les raphides restent dans le résidu. Ce résidu est remis en suspension dans un volume d'eau égal à celui du suc qui l'a fourni; il est aussi peu actif que le suc fermenté (expériences sur cobayes).

d) Expériences avec le suc frais fittré (sur papier).

Le suc frais, aussitôt après son expression, est filtré sur papier à la trompe, et se trouve par là privé de raphides, mais contient autant d'alcaloïde et de saponine que le suc non filtré.

Exp. XXI. - Cobaye mâle, neuf. Poids 527 gr.

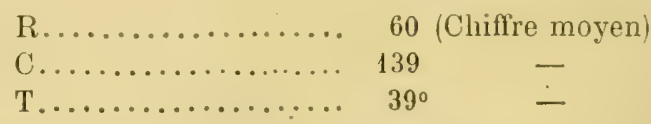

2 h. - Par sonde œsophagienne $20^{\mathrm{c} 3}$ du suc ci-dessus.

2 h. 30 R.............. 64 (Chiffre moyen)

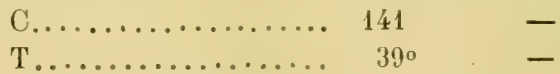

$3 \mathrm{~h}$. - Respiration et cour revenus à la normale. Le lendemain état général bon, pas de diarrhée.

Exp. XXII. - Entièrement comparable à l'expérience ci-dessus. 
Donc $20^{c 3}$ de sue frais filtré sont à peine actifs.

Exp. XXIII. - Colbaye, femelle, neuf. Poids $820 \mathrm{gr}$.

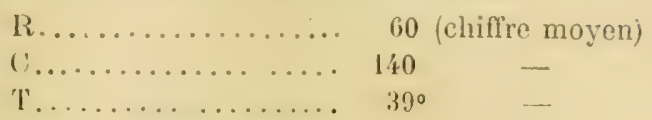

I h. - Dans l'estomac $33^{\circ 3}$ du suc ci-dessus.

1 h. 30 R.............. 63 (Chiffre moyen)

C.............. $142 \quad-$

2h. R.............. 70 -

C.............. $145 \quad-$

T............. $38^{\circ} \quad-$

Un peu de respiration périodique, d'angoisse, d'inertie motrice.

2 h. 35 - Retour à la normale. Le lendemain, diarrhée légère qui persiste 24 heures. L'animal se rétablit.

Exp. XXIV. - Même cobaye. Le lendemain de son rétablissement, $10^{c 3}$ du mème suc. L'intoxication se déclare avec ses caractères, mais après deux heures d'accès dyspnéiques, alternant avec des périodes de calme, l'animal se rétablit.

Exp. XXV. - Cobaye femelle. Poids $800 \mathrm{gr}$., ayant résisté à l'ingestion de $37^{c^{3}}$. Reçoit dans l'estomac $20^{\mathrm{c} 3}$, le lendemain de son rétablissement; il meurt en $4 \mathrm{~h}, 1 / 2$ avec les symptòmes de l'intoxication progressive et mortelle, provoquée par le suc frais non filtré.

L'autopsie révèle les lésions déjả décrites.

Examen histologique du tube digestif (fixé aussitòt par le sublimé acétique).

Estomac. - Couche pseudo-membraneuse d'éléments épithéliaux desquamés, leucocytes, mucus abondant. La muqueuse est presque partout dépouillée de son épithélium. Cette chute épithéliale ne se localise pas aux plis, elle s'étend à l'orifice des glandes.

Congestion vasculaire, surtout au niveau des culs-de-sac glandulaires; infiltration leucocytique de tout le tissu conjonctif interglandulaire, surtout accusée près de la surface; les autres couches de l'estomac, normales.

Intestin grêle. - Presque partout, l'épithélium est desquamé, les débris de ces cellules et de leurs noyaux sont plongés, avec des leucocytes et quelques hématies, dans un magma muqueux, mucus très abondant avec globules sanguins dans les dépressions entre les villosités. La desquamation épithéliale s'étend, par endroits, à l'orifice des glandes, tout le tissu conjonctif des villosités présente une infiltration intense par les leucocytes; les autres couches de l'intestin, normales.

Exp. XXVI. - Un cobaye qui a résisté une première fois à l'adminis- 
tration stomacale de $20^{\mathrm{c} 3}$ de suc, et qui présente le lendemain une légère diarrhée, avec un état général en apparence satisfaisant, succombe en deux heures à l'ingestion stomacale de $7 \mathrm{c}^{3}$ de suc. Mèmes symptômes, mèmes lésions à l'autopsie que dans l'expérience ci-dessus.

e) Expérience avec le suc filtré, soumis à l'ébullition.

Ce suc est privé de toute trace d'alcaloïde; sans relater le détail des expériences, il suffit d'indiquer que des quantités très considérables peuvent être ingérées, par voiestomacale, sans déterminer le moindre accident. C'est ainsi qu'un cobaye, de poids moyen, supporte impunément l'ingestion de 100 et même $1500^{c 3}$ de ce liquide. La toxicité du suc filtré, soumis ì l'ébullition, peutêtre considérée comme pratiquement nulle, par voie stomacale.

\section{B. - Essais d'intoxication par voie sous-cutayée \\ a) Expériences avec le suc brut.}

De petites quantités, 2 à $3^{\mathrm{c} 3}$ de suc de parties vertes, exprimées à la presse, non filtrées, suffisent à déterminer, en injection souscutanée, des abcès, à évolution rapide, au point d'inoculation. Comme, dans ce cas, la mort peut toujours être attribuée, si clle n'est pas immédiate, à une infection et non à l'intoxication, nous avons renoncé à nous servir du sue non stérilisé. La présence des raphides ne peut en rien modifier l'absorption des principes solubles par le tissu cellulaire ; il est donc tout indiqué de les éliminer, on y parvient par la filtration. Voici quelques essais avec le suc filtré, non stérilisé :

\section{b) Expériences avec le suc filtré, non stérilisé.}

Exp. XXVII. - Cobaye mâle, neuf. Poids $430 \mathrm{gr}$.

$10 \mathrm{~h} .-5^{\mathrm{c} 3}$, sous la peau de la cuisse. Injection douloureuse,

$$
\begin{aligned}
& \text { R........... } 60 \text { (chiffre moyen). } \\
& \text { (........... } 139 \text { - } \\
& \text { T........... }
\end{aligned}
$$

10 h. 10. - L'animal mordille le point injecté, qui est évidemment le point de départ de sensations douloureuses. Agitation, anxiété, dyspnée légère (trahie par des eflorts d'inspiration; museau relevé, narines agitées de mouvements convulsifs; semble chercher à humer l'air). 


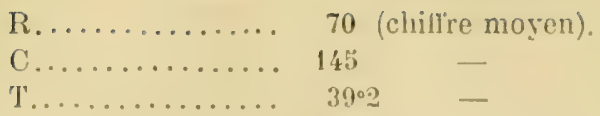

10 h. 20. - La dyspnée se dissipe peu it peu, le train postérieur de l'animal est un peu trainant, réactions motrices légèrement diminuées.

11 h. - L'animal se rétablit et est en parfait état le lendemain.

Exp. XXVIII. - Cobaye femelle, neuf. Poids 330 grammes.

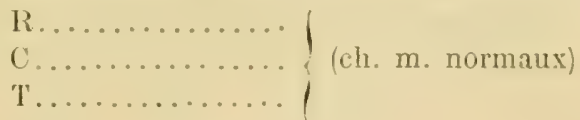

4 h. 10. - y $60^{3}$ en deux fois, sous la peau du dos. Injection rlouloureuse.

4 h. 20. - Anxiété, aritation, cour et respiration accélérés.

\& h. 30. - Respiration périodique (grandes inspirations à intervalles réguliers), polypnée, tremblement, chute sur le còté.

$$
\begin{aligned}
& \text { R............ } 76 \text { (chiflre moyen). } \\
& \text { C........... } 146 \quad \text { - } \\
& \text { 'T'......... } 399^{\circ} 3 \text { - }
\end{aligned}
$$

Peu à peu l'état comateux se déclare, la sensibilité générale diminue, les réactions motrices disparaissent, la respiration se ralentit, le cour faiblit.

4 h. 45. - Coma complet, intermittences respiratoires et cardiaques; après quelques petites convulsions, la mort survient dans une convulsion finale.

\& h. 47. - L'autopsie, faite aussitôt, révèle, du côté des poumons, du caur et les organes abdominaux, les mèmes lésions que dans l'intoxication par voie stomacale, mais ici ce sontles lésions pulmonaires qui sont particulièrement nettes. Quelques arborisations rasculaires et de petites suffusions sanguines sont les seules altérations à relever du còté du système digestif. Au point d'inoculation, colème gélatineux, rosé, fort étendu par places, réritable hémorrhagie dans le tissu cellulaire sous-cutané dorsal.

Exp. XXIX. - Deux autres expériences, dans des conditions tout ì fait semblables, donnent les mèmes résultats.

Le suc filtré détermine donc, par voic sous-cutanée, une intoxication mortelle, a dose beancoup phus litible que par vole stomiacale.

c) Expériences avec le suc stérilisé par filtration à la trompe (sur bougie en alumine, appareil de Kitasato).

Le suc, ainsi filtré à froid, contient ia la fois l'alcaloïle et la saponine. 
Il est inutile de répéter pour lui ce qui vient d'être dit, à propos du suc non stérilisé, simplement filtré sur papier; son activité est plus faible cependant que celle de ce dernier, ce qui s'explique si l'on admet que frar une filtration rapide, sous failhe pression, une partie des principes solubles ne traverse pas la bougie.

d) Expériences avec le suc filtré, stérilisé à l'autoclave, à $120^{\circ}$.

On peut injecter impunément sous la peau du cobaye, du lapin et du chien des quantités relativement considérables de ce suc, sans déterminer aucun accident notable; on évite même toute formation d'abcès si l'on s'assure de la facon aseptique dont est pratiquée l'injection, si ou la pratique lentement, et en des points différents du corps.(pour les doses élevées).

\section{C. - Essais d'intoxication par vole péritonéale.}

a) Expériences avec le suc filtré, non stérilisé.

Exp. XXX. - Cobaye mâle, neuf, poids $480 \mathrm{gr}$.

2 h. 20. $-5^{{ }^{3} 3}$ dans le péritoine.

2 h. 40. - Aucun symptòme assez net d'intoxication ne s'étant manifesté (sauf une douleur assez vive devant partir du point d'inoculation), 5e3 nouveaux, dans le péritoine. Les symptômes de l'intoxication apparaissent, et celle-ci se développe progressivement, comme il a été dit pour l'intoxication par voie hypodermique.

3 h. 35. - Mort.

Autopsie immédiate. Les lésions sont celles précédemment relatées; les altérations pulmonaires attirent spécialement l'attention, mais, tandis que par voie hypodermique, le toxique ne déterminait qu'une congestion peu intense $d u$ tube intestinal, ici les anses intestinales, voisines du point d'inoculation, présentent de larges suffusions sanguines, en particulier sur une notable portion de la longueur du côlon.

La mort, en raison même de sa rapidité, ne peut être mise sur le compte d'une infection; l'examen bactériologique et les cultures démontrent aussi l'absence de péritonite septique.

Il est peut-être cependant bon de ne pas attacher autant d'importance aux essais, dans lesquels la voie péritonéale a été choisie comme voie d'absorption, en raison de l'irritabilité si grande de cette séreuse, et des réflexes pulmonaires et circulatoires, dont elle peut être le point de départ, après injection d'un liquide aussi irritant que lé suc d'Arum. 
b) Expériences avec le suc filtré, stérilisé.

1. A la trompe sur bougie. Nous constatons encore que la filtration ì la bougie diminue quelque peu la toxicité du suc.

2. Al loutorlare. Ce sue peut être injecté impunénent, it doses assez fortes, dans le péritoine (10 à $1 \mathrm{ă}^{\circ 3}$ lapin, 20 à $30^{c 3}$ chien).

D. - Essais d'intoxication par voie intra-veineuse.

a) Expérience avec le suc filtré, stérilisé à l'autoclave, à $120^{\circ}$.

Lapin.

$30^{\circ 3}$ sont supportés, sans désordres appréciables, par le lapin, chez lequel l'injection est poussée, arec une lenteur suffisante et is plusieurs reprises, dans la veine marginale de l'oreille. Le mème animal supporte l'injection de $100^{\circ 3}$ du même suc, en 3 fois, dans les 24 heures, sans rien présenter d'anormal. On peut done dire que le suc filtré, stérilisé, est lépourvu de toute activité toxique, mème en injections intra-veineuses, chez un animal aussi sensible que le lapin, chez lequell'arrivée d'une substance, mème faiblement toxique, au contact de l'endocarde, peut déterminer des réflexes mortels.

Chien.

L'expérience sur le chien conduit au même résultat.

\section{Essais avec l'Extrait alcoolique.}

L'examen chimique nous ayant montré que les fruits mûrs d'Arum sont un peu plus riches en saponine et en alcaloïde, nous arons préparé avec le fruit total (péricarpe et graines un extrait alcoolique, sirupeux, d'un brun orange, miscible à l'eau en donnant une émulsion trouble précipitation de la saponine), acide, suffisamment fluide lorsqu'il est de préparation récente, pour ètre injecté sans dilution par roie hypodermique. L'épuisement par l'alcool à l'ébullition, puis par éraporation dins le vide, à température relativement basse ( $73^{\circ}$ environ), assure la dissolution totale de la saponine. Quant à l'alcaloöde, si les fruits ont été conservés un certain temps en bouteille, même recouverts d'alcool, on peut admettre 
qu'une partie de l'alcaloïde, si altérable, s'est partiellement décomposée.

La conicine émet beaucoup de vapeurs ì une température inférieure à $100^{\circ}$, mais ne se volatilise qu'à $187^{\circ}, \check{i}, 189^{\circ}$ et mème $217^{\circ}$, selon les divers auteurs.

Donc, l'extrait préparé avec l'alcool à l'ébullition, c'est-d̀-dire à la température de $70^{\circ}$ environ, a pu etre privé d'une petite partie de sa conicine, mais la majeure partie doit y être restée.

La vapeur d'alcool, comme la vapeur d'eau, doit entraîner la conicine, mais la décomposer partiellement. Lalcaloüde entrainó par la vapeur d'alcool retombera bien dans l'extrait, avec la vapeur condensée dans le réfrigérant, mais, de ce chef, il pourra encore y avoir perte d'une partie de l'alcaloïde.

L'extrait alcoolique qui a servi aux essais ci-dessous contient donc une notable-proportion de saponine d'Arum, une quantité faible d'alcaloide, ct une quantiti extrimement fiable de sels de $\mathrm{l}$ (très légèrement solubles dans l'alcool étendu employé).

\section{Grenouille.}

Exp. XXXI. - Grenouille mâle, neure, virace ('T. 13․ P. 19 gr.).

1 h. כ̈. - V gouttes de l'extrait ci-dessus, non dilué, dans le sac lymphatique dorsal; agitation spontanée.

\& h. 10. - Inertie; les sauts provoqués par les excitations dénotent une inertie marquée du train postérieur, sensibilité à la pression et à la température (écrasement et carbonisation au fer rouge) totalement abolie dans les membres postérieurs, et nettement affaiblie dans les antérieurs; mouvements spontanés rares, pénibles; addition latente des excitations; après plusieurs excit. successives, simple ébauche de mouvements. Les pattes postérieures, mises en extension, cessent de se replier. Inertie complète de l'animal, les pattes étendues, aplati sur la table, sensibilité générale (tactile et thermique) totalement abolie, absence de tout mouvement de déglutition de l'air.

4 h. 30. - Quelques mourements spontanés filbrillaires; très rares et très pénibles mouvements de déglutition.

5 h. - Réllexes cornéens indiquant seuls la persistance de la vie:

5 h. 20. - Réflexes cornéens à peine perceptibles.

5 h. 45 . - Mort. 
le myocarde, par le contact de l'extrait acide.A chaque instillation, le point touché blanchit, et parait présenter une contraction locale plus énergique et fugace.

\section{Action sur les nerfs.}

La diminution de la sensibilité, chez la grenouille intoxiquée par l'extrait, commence par les membres postérieurs, puis s'étend aux antérieurs et à toute la surface cutance. Il existe normalement une région du corps, à l'excilation de laquelle l'animal réagit énergiquement, c'est celle de l'anus. Dans l'intoxication confirmée, elle est parfaitement inexcitable.

L'absence de réflexes peut tenir: $1^{\circ}$ à ce que l'excitation périphérique ne peut plus cheminer vers la moelle, 20 à ce que la réaction des centres réflexes ne se produit plus, $3^{\circ}$ à ce que l'excitation centrifuge, partie de ces centres, ne peut plus cheminer vers les muscles. Deux hypothèses restent encore: il y a inhibition exercée sur les centres réflexes inférieurs par les centres encéphaliques; ou bien le poison est un agent curarisant : il agit sur la plaque motrice, et les conducteurs nerveux, ainsi que les centres, peuvent rester intacts, bien que le mouvement de réaction ne puisse plus se produire.

A la période l'état de l'intoxication, toute sensibilité a disparu; mais les divers ordres de sensibilité disparaissent successirement. Lorsque la sensibilité cutanée à la pression a disparu, la sensibilité thermique persiste encore. Lorsque cette dernière a disparu à son tour, l'excitation électrique donne encore des résultats.

Ceci n'a pas lieu de nous étonner. Sans s'arrêter de préférence à l'une ou ì l'autre des hypothèses concernant les sensibilités spéciales, leurs voies de conduction nerveuse diffé rentes, et la spécialisation des corpuscules, il est facile de comprendre que l'ébranlement moléculaire,provoqué par une compression mécanique,est tout à fait hors de proportion arec l'ébranlement provoqué par les vibrations calorifiques. Par la même raison, l'excitant électrique sera encore efficace, lorsque l'excitant thermique aura cessé d'ètre assez énergique. Les excitants chimiques caustiques agissent encore beaucoup plus 
tard. Il importe de remarquer que, sitôt la sensibilité cutanio disparur, l'excitation direrte des muscles el destendons provorur les riflexes que l'excitation cutanéc est désormais impuissante a joroduire.

Mais cette localisation de l'anesthésic aux corpuscules sensibles cutanés n'est bien mise en évidence que par l'injection de faibles doses du toxique. Dans ces ronditions, on pent interrosere la sensibilité d'une faron continue, à mesure que les plrimomines toxiques s'accentuent.

Après l'injection d'une dose massive, l'anesthésie envahit d'un seul coup les terminaisons nerveuses des muscles et des fondons, en même temps que celles de la peau.

Au slade d'anesthésic générale confirmée, l’excitation ilurlrique directe du sciatique provoque encore des contractions réflexis. Ces simples constatations mettent bien en evidence la localisation primilive du poison sur les corpuscules sensibles périphériques, et non sur la substance médullaire ou les plaques motrices. Lepoison est done, sous ce rapport, quelque peu analogue à la cocaïne. Il existe cependant une grande diffirence entre les deux agents. La cocaïne. instillée dans l'œil, anesthésie la cornée; il n'en est pas de même pour l'extrait d'Arum, et, dans l'intoxication qu'il détermine, la sensibilité cornéenne persiste jusqu'à la mort.

Dans l'intoxication confirmée, les centres médullaires sont d'ailleurs touchés, une excitation „légère et directe du sciatique est impuissante à provoquer un réflexe; une série d'excitations successives le déterminent au contraire, après une phase d'addition latente. Un peu plus tard, une excitation, unique mais forte, ne provoque pas une réaction immédiate, le réflexe ne s'effectue qu'après quelques secondes.

Ces phénomènes de retard, très nets, prourent, non pas tant que la transmission par les conducteurs nerveux se fait mal, mais plutit que les centres nerveux sont atteints. Leur réaction est lente et, pour la produire, l'addition latente de plusieurs excitations faibles est nécessaire.

Sur l'animal normal, les centres encéphaliques exercent une ac- 
tion modératrice sur les centres médullaires. L'intoxication n'aurail(llo pas pour résultat d'augmenter cotte action modératrice des centres supérieurs, c'est-à-dire d'inhiber la moelle?

Pour vérifier celte hypolhèse, il n'y a qu'à opérer sur un animal dont le bulbe est sectionné. On sait que, dans ces conditions, l’irritabilite réllexe est augmentée. Cependant, sur la grenouille intoxiquée, a bulbe sectionné, on observe la meme diminution de l'excitabilité des centres médullaires, à mesure que l'intoxication se déroule. Même peu de temps avant la mort, il est facile de constater, par l'excilation centrifuge du sciatique. que le phénomène curarique, si fréquent dans la phase terminale de beaucoup d'intoxications, fait ici défaut.

L'excitabilité des plaques motrices et du muscle persiste donc.

Cobaye.

\section{A. - Par vole soles-cutanée}

Des essais d'intoxication par roie hypodermique ne nous ayant pas donné de résultats assez nets, pour utiliser au mieux la quantité restante d'extrait que nous possédions, nous avons réalisé chez le cobaye l'intoxication par voie péritonéale.

\section{B. - Par voie péritoxéale}

Exp: XXXIV. - Cobaye mâle, neuf..... Poids 598 gr.

$$
\begin{aligned}
& \text { R.............. } 60 \text { (chiffre moyen) } \\
& \text { T.............. } 39^{\circ}
\end{aligned}
$$

3 h. 35. - Avec toutes les précautionsantiseptiques, 3 c $^{3}$ d'extrait dilué (à parties égales d’eau-stérilisée) dans le péritoine. Aussitôt, parésie du train postérieur, allure hyénoïde: l'animal est inerte, les pattes postéricures étendues. Diminution de la sensibilité tactile et de l'intensité des réflexes, addition latente des excitations.

3 h. 40. - L'anesthésie, complete atux membres postérieurs, envahit les antérieurs, puis s'étend à tout le corps.

3 h. 50 . - Une très forte compression des pattes postérieures reste inefficace à provoquer toute réaction. Pattes antérieures ne réagissant à cette compression que par des mourements fibrillaires, incapables d'opérer le retrait du membre. L'oreille peut ètre coupée sans réaction, la sensibilité au contact d'un fer rouge est considérablement diminuée, 
presque nulle aux membres postérieurs. L'animal, tombé sur le côté, ne se relève qu'avec peine, respiration saccadée, haletante (68), cœur encore bien frappé (143).

4 1. 5. - Urine faiblement, rétablit mieux son équilibre, lorsqu'on le déplace, l'anesthésie persiste.

4 h. 20. - Urine à nouveau, mème état.

4 h. 55. - Les réactions reparaissent, après excitation des pattes antérieures, des oreilles; anesthésie persistante aux membres postérieurs. Quand l'animal déplacé rétablit son équilibre, il est atteint pendant quelques instants d'un tremblement caractéristique (oscillations horizontales autour de l'axe lorgitudinal du corps). Réflexes rotuliens très aflaiblis.

5 h. 20. - Même état, bien que la sensibilité douloureuse à la pression reparaisse.

5 h. 40. - A l'anestliésie, succède de l'hyperesthésie.

Lelendemain matin, l'animal a le poil hérissé, les yeux chassieux, l'abdomen douloureux à la pression; il présente de l'hyperesthésie cutanée généralisée, il pousse le faibles plaintes continues. La motricité et la sensibilité sont revenues à la normale. Il a évacué des urines abondantes et des féces fermes. La période d'intoxication est incontestablement passée, et de la péritonite doit évaluer.

Mort à 2 heures de l'après-midi.

Autopsie immédiate. Péritoine. - Brides fibrineuses, quantité considérable de sérosité rousse (couleur del'extrait); dans tous les culs-de-sac péritonéaux, dépòts pulvérulents dus à l'extrait. L'ensemencement de la sérosité de l'ascite, sur divers milieux de culture, à l'étuve à $28^{\circ}$, montre l'absence totale d'infection.

Tube digestif. - normal.

Vessie.-Distendue.-Rein. Glomérulite très nette, congestion.

Capsules surrénales. - Zone médullaire très congestionnée. Foie congestionné, friable.

Poumions. - Asphyxiques, larges plages de teinte violacée hortensia; suffusions sanguines surtout au sommet.

Cœur. - Ventr. g. en systole, vide de sang, or. g. gorgée de caillots noirs, ventr. d. en diastole, dilaté par des caillots, ainsi que l'or, d. et les grosses branches veineuses qui en partent.

Exp. XXXV et XXXVI. - Deux autres expériences, dans les mèmes conditions, donnent les mèmes résultats.

Chez le cobaye, la toxicité de l'extrait n'est pas suffisante pour tuer, à dose relativement forte $10 \mathrm{c}^{3}$, par voie hypodermique. Administré par celle voie, il détermine la formation d'un abcès aseptique. 
La dose mortelle par voie péritonéale est de ö $\mathrm{e}^{3}$. L'intoxication se diclare alors aussitit, elle est essentiellement caractérisée par de l'anesthésie (localisation du toxique sur les terminaisons nerve'uses, une diminution d'excitabilité réllexe des centres médullaires; la localisation du toxiquesur ces centres semble débuter par les parties postérieures de l'axe spinal, et remonter progressivement vers la partic antérieure; l'allure hyénoïde, le tremblement caractéristique indiquent que les centres médullaires sont nettement influencés par le poison. Les modifications du rythme et l'amplitude des muuvenents respiratoires ne sont que passigères et de peu d’intensité. Il en est de même pour le cœur.

La mort est très certainement due non à l'intoxication, mais à l'irritation péritonéale, aseptique, dont la cause principale peut être due, au moins en partie, à l'acidité de l'extrait injecté; 12 heures après le début de l'intoxication, les symptômes de celleci ont disparu, vraisemblablement après que l’animal s'est déharrassé. par plusieurs mictions, du toxique absorbé. Il est remarquable de constater l'absence à l'autopsie de toute lésion appréciable du tube digestif, alors que les lésions sont si nettes, mème dans l'intoxication par voie péritonéale, à l'aide du suc frais. Or, dans l'extrait, la quantité d'alcaloỉde est extrêmement faible, le principe dominant est la saponine. Bien des symptômes observés concordent assez bien avec ceux que détermine l'intoxication par une saponine toxique.

Lapin.

\section{PAR VOIE PÉRITONÉALE}

Exp. XXXVII - Lapin neuf. Poids 2 k. 500.

$10 \mathrm{~h}$. - Avec toutes les précautions antiseptiques, 2, 5ّ3 d'extrait alcoolique (dilué à parties égales d'eau stérilisée) dans le péritoine. La contraction cardiaque est enregistrée avant, et une $1 / 2$ heure après l'injection, par le cardiographe Bardier.

Les tracés, pris d'une façon ininterrompue, ne révèlent aucune altération du rythme cardiaque. Pendant toute la durée de l'expérience, l'animal ne présente aucun symptòme d'intoxication.

L'extrait alcoolique n'agit donc pas sur le cour du lapin, et ne 
détermine à doses modérées aucun phénomione appreceiable d'intoxication.

Chien.

\section{PAR VOIE HYPOIERMIQUE}

L'injection de substances, même irritantes, dans. le tissu cellulaire sous-cutané chez le chien, est en général fort bien supportée. I a possibilité de faire absorher assez rapidement des quantités, relativement considérables, de toxique, et d'éviter, contrairement à ce qui a lieu dans les essais sur le péritoine, tout retentissement réflexe sur les grandes fonctions de l'organisme, nous engage à expérimenter l'action de l'extrait sur le chien, par voie hypodermique, à doses fortes.

Exp. XXXVIII - Jeune chiot (2 mois 1/2). Poids 2 kilos.

4 h. - Dans la masse musculaire externe de la cuisse gauche, XII gouttes d'extrait non dilué. Injection douloureuse. Le membre injecté est trainant, l'animal lèche et mordille le point de l'injection; il ne se déplace ru'en trainant la patte du côté injecté.

4h. 10. - Se déplace avec agilité, tout symptòme local a disparu.

4 h. 20. - XL gouttes au même point; douleur locale, membre trainant; mais, au bout de quelques minutes, tout symptôme net a disparu successivement.

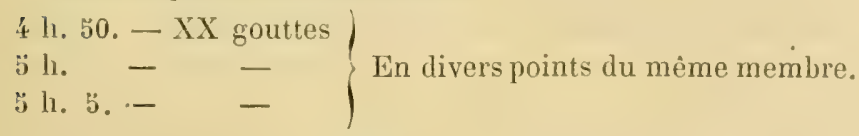

La jambe injectée est quelque peu crémateuse et claudicante, mais aucun symptòme général. Le lendemain l'animal est bien portant, mais évite de porter sur la patte injectée. Les jours suivants, abcès.

Exp. XXXIX - Sur le même animal, totalement rétabli au bout de 2 jours, ne présentant aucun abcès local au membre précédemment injecté, la même expérience est répétẻe dans des conditions identiques sur l'autre membre ; mêmes résultats, absence de toute complication. ultérieure générale; abcès aseptiques aux points inoculés. Ces abcès s'évacuent spontanément et l'animal se rétablit parfaitement.

Un chien de deux liilos supporte done impunément $6^{\mathrm{c} 3}$ d'extrait, par roie hypodermique, sans manifestations générales. Il en est de même, avec des doses plus fortes, injectées par fractions, en des points différents du corps, jusqu'à $1 \dddot{J}^{3}$, mais à chaque point injecté évolue un abeis ou phlegmon, aseptique, qui s'évacue spontané- 
ment, avec rapidité, la plaie ainsi produite se cicalrise rapidement. On roit par lit la faible toxicité de cel extrait. On ne peut pas arec semblables doses parler de véritable intoxication. Tout au plus y a-t-il des troubles locaux aux points d'injection, diminution de la sensibilité et impotence fonctionnelle.

\section{Essais avec l'alcaloïde.}

La toxicité de l'alcaloïde d'Arum n'est pas très forte, ainsi que le montrent les expériences ci-dessous.

Nous arons préparé, en partant de l'alcalö̈de impur (mélangé de produits goudronneux), une solution sulfurique, forcément très diluée; chaque goutte de cette solution contient au maximum 2 milligrammes (chiffre rond) de sulfate d'alcaloïde.

Cobaye.

\section{PAR VOIE IIYPODERMIQUE}

Dans une incision faite à la peau du dos d'un jeune cobaye de 340 grammes, on place une petite boulette de quelques grammes d'alcaloïde impur, mélangé à des produits goudronneux; au bout. d'une heure, il présente de la parésie du membre injecté, mais il se rétablit rapidement, sans présenter d'autre symptôme d'intoxication.

On injecte sous la peau, comparativement, à deux cobayes sensıblement de mêma poids (600 grammes) : $1^{0}$ a l'un, $1^{c^{3}}$ d'une solution desulfate de cicutine; $2^{\circ}$ à l'autre, $1^{13}$ d'une solution de sulfate d'alcaloïde d'Arum (ces deux solutions étant de même concentration). Le premier cobaye meurt, au bout de ̋̈ minutes, en paraplégie, par asphyxie due à l'arrêt des mouvements respiratoires; le second cobaye ne semble en rien gêné.

La toxicité de l'alcaloïde d'Arum est donc beaucoup plus faible que celle de la cicutine type.

PAR VOIE STOMAGALE

L'ingestion, par voie stomacale, de 2 grammes d'alcaloïde impur, détermine la mort, chez le cobaye, avec les symptômes relatés à propos de l'intoxication par le suc. Dans cette expérience, 
la cessation des mouvements respiratoires se produit très vite, et, à l'autopsie, on trouve les mèmes lésions que cellés relatées ei-dessus (à propos de l'intoxication par le suc), mais ces lésions sont beaucoup moins accusées sur le tube digestil.

N'ayant à notre disposition qu'une quantité très restreinte d'alcaloïde, nous avons surtout cherché, par quelques essais, it nous assurerdes analogies ou des différences qu'il présente, au point de vue physiologicque, avec la cicutine.

\section{Grenouille.}

$1 / 2^{e 3}$ de solution de sulfate de cicutine tue une grenouille en 23 minutes, en déterminant la paralysie du membre injecté; $1 / 2^{\text {e3 }}$ de la solution sulfurique neuiralisée d'alcalö̈de, sensiblement au mème litre, détermine aussi chez une grenouille, sensiblement de même poids, la paralysie immédiate du membre injecté, mais l'animal se rélablit au bout de quelques heures.

\section{Souris.}

En raison de la faible masse de son corps, la souris se prète bien à des essais avec de faibles quantités de substance. Elle est, d'ailleurs, très sensible à la ciculine.

Exp. XL. - Souris blanche, neuve, du poids de 22 gr.

4 h. 30. - I goutte de la solution alcaloïdique, sous la peau du llanc; lèche et mordille aussitòt la place de l'injection; respiration haletante; traine un peu les membres postérieurs.

4 1. 40. - III gouttes dans les muscles delacuisse; paralysie immédiate du membre injecté, se déplace en trainant ce membre, la patte renversée; seuls, les muscles de la hanche qui ont échappé au contact direct de la solution, jouissent encore de leur motricité; la sensibilité de la patte paralysée persiste, même état jusquu"a la nuit. La souris est morte le lendemain matin.

Autopsie.- Edème gólatineux, sanguinolent, au point d'inoculation; l'injection n'a déterminé aucun traumatisme des nerl's, mais a déposé le toxique immédiatement au voisinage du sciatique, d’où paralysie immédiate.

Vessie. - Distendue.

Rein. - I'iqueté de glomérulite à la surface, encore plus net sur la coupe tangentielle, la coupe longitudinale de l'organe donne passage en abondance à du sang noir liquide. 
Tube digestif. - Légère congestion.

Foie. - Congestionné, friable.

Poumons. - Asphyxiques, avec 2 ou 3 larges taches ecchy. motiques, noirâtres. au sommet.

Cœur. - C. dr. gorgé de sang noir, encore un peu fluide, ainsi que les grosses veines, qui en partent, et l'or. g., ventr. g. en systole, vide de sang.

\section{Oisernu.}

L'intoxication des oiseaux par la ciculine offre des phénomènes assez caractéristiques; il est donc indiqué d'essayer sur un oiseau de petite taille l'action de l'alcaloïde d'Arum.

Exp. XLI - Moineau. Poids 327 grammes.

9 h. 30. - Dans la masse des pectoraux gauches, $V$ gouttes de la solution alcaloïdique.

9 h. 3ร. - Agitation spontanée marquée.

$10 \mathrm{~h}$. - A cette période d'agitation, succède une période de calme, presque d'inertie, mais sans symptòmes nets d'intoxication,

10 h. 45. - XX gouttes au même point. Immobilité, respiration haletante.

10 h. 47. - Agitation.

11 h. 10. - X gouttes dansla masse pectorale du côté opposé, polypnée, le corps tout entier est agité de soubresauts continus et rythmiques.

1) h. 15. - Polypnée intense, l'animal lutte nettement contre l'ásphyxie; secousses convulsives; chute en avant. Mort.

Autopsie immédiate.

Rein. - Congestionné.

Foie. - Id.

Tube digestif. - Légère congestion.

Poumons. - Congestion intense, localisée au sommet, presque de l'hémorragie.

Cœur. - Ventr. vide ne réagissant plus aux excitations, oreill. dilatée par un sang fluide vermeil, réagissant encore à la piquire.

Un adème gélatineux rouge brun, presque hémorrhagique, s'étend à la surface et dans la profondeur des masses pectorales avec une intensité des plus notables. Le traumatisme fait par l'aiguille de la seringue est tellement insignifiant qu'il est impossible d'en trouver trace. 


\section{CIIAPITRE IV}

\section{ÉTUDE EXPÉRIMENTALE DES PROPRIÉTÉS TOXIQUES DE L'ARISARUM VULGARE}

Aucune recherche expirimentale sur les effets physiologiques et toxicologiques d'Arisarum vulgare n'a été tentée jusqu'ici.

L'Arisarum vulyure étant une plante exclusivement méridionale, nous n'avons pu nous procurer qu'une quantité relativement faible (quelques kilos) de plante fraiche. Nos résultats sont done forcément incomplets; ils comblent cependant, dans une certaine mesure, les lacunes de nos connaissances sur cette plante toxique.

\section{essais d'intoxication par vole digestive}

Tous nos essais ont été tentés avecle suc extrait des parties vertes aériennes (plantes expédiées de Nice en décembre et janvier), à la période de floraison ou de fructification.

\section{Suc extrait des parties aériennes.}

a) Suc exprimé à la presse de la plante fraîche, non filtré (c.-à-d., riche en raphides).

Cobaye.

Exp. XLII. - Cobaye mâle, neuf. Poids $830 \mathrm{gr}$.

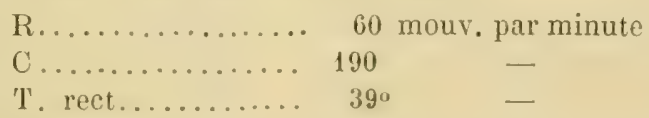

2 h. 50. - Ingestion par la sonde osophagienne de $10^{\text {c } 3}$ de suc.

2 h. 53. - Dyspnée, R. 80, C. 143.

2 h. 60. - Polypnée (1), R. 96.

(1) On serait, peut-être, tenté de nous reprocher le terme de polypnée, appliqué à une augmentation de fréquence de la respiration ne dépassant pas le double de sa valeur normale; mais ce terme a le grand avantage d'être bref. 
C. 150, T. rect. $38^{\circ}$.

Aucun effort d'expulsion.

3 h. - R. 80 , mouvements respiratoires de faible amplitude.

C. 142 . T. rect. $36^{\circ}$.

3 h. $30,-$ R , 72.

C. $13 \%$.

Pendant toute la durée de l'expérience, la motricité et la sensibilité sont restées intactes. Le lendemain, l'animal est rétabli. Il a mangé avec appétit, n’a pas eu trace de diarrhée.

Exp. XLIII. - Colbaye mîle, neuf. Poids 900 gr.

$$
\begin{aligned}
& \text { R............. } 62 \text { (chiffre moyen) } \\
& \text { C............. } 138 \text { - } \\
& \text { T. rect.......... } 37^{\circ}
\end{aligned}
$$

10 h. 40\%. - Ingestion par la sonde osophagienne de $20^{\text {c3 }}$ de suc. (Une petite quantité tombe dans l'arrière-bouche, et peutêtre, sous l'inlluence du courant d'expiration, dans les fosses nasales; les mouvements d'expulsion de ces quelques gouttes de suc ne sont pas très énergiques, elles s'écoulent peu à peu, sans provoquer grande réaction, par de petits mouvements des narines.)

10 h. ว0. - Vivacité.

$11 \mathrm{~h}$. - Tremblement intense de tout le corps.

$$
\begin{aligned}
& \text { R............... } 76 \text { (chiffre moyen) } \\
& \text { C............. } 142 \\
& \text { T. rect............ } 36^{\circ} 5 \text { - }
\end{aligned}
$$

11 h. 20. - Urine à plusieurs reprises.

Un peu d'inertie.

$$
\begin{array}{lr}
\mathrm{R} \ldots \ldots \ldots \ldots \ldots \ldots & 80 \\
\mathrm{C} \ldots \ldots \ldots \ldots \ldots & 189 \\
\mathrm{~T} . \operatorname{rect} \ldots \ldots \ldots \ldots & 36^{\circ}
\end{array}
$$

12 h. - Retour à l'état normal, mange avec appétit, le lendemain n'a présenté qu'un peu de diarrhée. Pendant toute la durée de l'expérience, l'animal n'a présenté de troubles ni de la sensibilité, ni de la motricité.

Exp. XLIV et XLV. -- Entièrement comparables aux deux précédentes, leur relation est superflue.

Exp. XLVI. - Même cobaye que dans l'expér. XLIII.

Ce cobaye, deux jours après son rétablissement parfait en apparence, est remis en expérience.

Son poids a diminué. Poids 820 grammes.

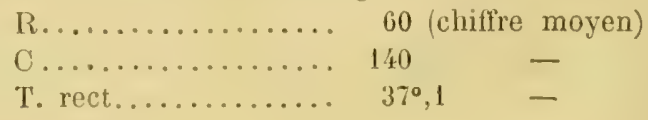


10 h. 53. - Ingestion par la sonde rrsopluagienne de $3^{3}$ de suc (la sonde n’est enfoncée qu'it mi-longueur de l'nesophage), une petite partie du suc reliue, par mouvements péristaltiques, dans le pharynx.

Pendant toute l'injection, qui semlle fort douloureuse, plaintes, mourements de déglutition à rebours, salivation. Quelques gouttes refluent par les losses nasales, et jrovoquent des réllexes d'exjulsion.

Signes de violente irritation du pharynx.

11 h. - Agitation, déglutition it rehours, écoulement de mucus, par la bouclie et les fosses nasales.

11 h. 13. - Court accès de polynnée. R. 68; C. 183; T. 370; tremblements. P'uis période de calme et d'inertie.

12 h. - Nouvel accès de polypnée intense. R. 76; C. 150 ; T. $36^{\circ}, 8$. Un tremblement violent s'etend it tout le corps. Peu à peu, l'animal tombe dans le coma qui s'accentue à 1 heure.

$1 \mathrm{~h}$. - Respiration ralentie, 60 ; cour également ralenti, 110 , devenant à peine perceptible, puis intermittences cardiaques et respiratoires; des inspirutions hrusques, brèves, profondes, suivies d'expirations purement passives, alternent arec des pauses de plus en jilus prolongées. (v. tracés, p. 130). Sensibilité et motricité presque disparues.

1h. 15. - Coma complet, arrèt de la respiration; le cour bat encore faiblement après l'arèt de la respiration, puis cesse d'être perceptible, sensibilité générale et motricité disparues, sensibilité cornéenne très aflaiblie, quelques petites convulsions, suivies diune convulsion finale.

1 h. 17. - Mort.

Autopsie ( $\mathrm{l}$ h. après la mort).

OEsophage congestionné, recouvert de mucus abondant.

Estomac rempli d'aliments, modérément distendu par des gaz, congestion peu accuséc; la muqueuse, débarrassée par lavage des mucosités et des particules alimentaires adhérentes, s'exfolie par plages, par le simple frottement du doigt (il ne peut étre ici question d'une autodigestion); par plages elle est plus adhérente, livide, d'aspect pultacé.

Intestin. - Tout l'intestin grèle, surtout le duodénum, présente une teinte hortensia, sa cavité est distendue par un liquide séreux, sanguinolent, çì et là des mucosités abondantes. L'examen microscopique rérèle la présence, dans ce liquide, de leucocytes, d'hématies, de cellules épithéliales, et de nombreux raphides.

$A u$ niveau du ciecum, une masse alimentaire volumineuse a arrêté le cours du liquide toxique. Celui-ci n'a pas péné- 
tré dans le gros intestin, qui est normal, et renferme des bols fécaux bien moulés.

Rein. - Piqueté violet à la surface, peu accusé, sur la coupe longitudinale passant par le hile; congestion nette de la zone des pyramides: sur la coupe tangentielle de la région corticale, piqueté rouge violacé (congestion glomérulaire).

Vessie. - Pleine.

Organes génitaux internes. - Congestionnés.

Foie. - Congestion légère, à la coupe, laisse sourdre des gouttelettes de sang noir.

Tout le systeme veineux abdominal est rempli d'un sang noir, fluide.

Poumons. - Asphyxiques, suffusions sanguines, par places de larges marbrures noires, infarctus extrêmement nets et larges (les lésions pulmonaires sont avec celles de l'intestin les plus saillantes). Ecchymoses sous-pleurales. A la coupe, noyaux hémorrhagiques, les petites ramifications bronchiques livrent passage à une spume abondante.

Cœur. - Cœur gauche vide de sang, ventricule en systole, cœur droit en diastole, rempli de sang noir, avec caillot ténu.

Artère pulmonaire, veines caves et leurs branches d'origine gorgées de sang noir fluide.

Encéphale. - Congestion des méninges; il en est de même du bulbe.

Exp. XLVII. - Même cobaye que dans l'expérience XLII. Soumis, 2 jours:après son rétablissement apparent, à une nouvelle injection stomacale du mème suc, il succombe avec une dose de $4 \mathrm{c}^{3}$.

Mêmes résultats, quant aux symptômes et aux lésions, que dans l'expérience XLVI.

Il est inutile d'insister sur les lésions délerminées par l'ingestion d'Arisarum, ce que nous avons dit de celles déterminées par l'intoxication à l'aide des Arum leur est exactement applicable.

Violente irritation des voies digestives, surtout de l'intestin; congestion asphyxique, tels sont les faits les plus saillants. Le mode de mort est l'asphyxie. Celte expérience nous révèle un fait intéressant. L'animal n'a subi, du fait d'une intoxication antérieure légère, aucune accoutumance. Il supporte une première fois $20 \mathrm{c}^{3}$ de suc, en ne présentant que des désordres passagers, son rétablissement est parfait en apparence, une perte de poids indique seulement un malaise quelque peu durable; l'absence de diarrhée, 
la conservation de l'appétil tendent à faire rearter lidece d'une irritation gastro-intestinale persistante. Lanimal succomles apries ingestion d'une quantité minime de suc : $3 \mathrm{c}^{3}$.

En présence de ces résultats, il est indiqué de voir si le suc d'Arisarum n'agit pas par une sorte d'accumulation.

Une première dose de $10^{\text {r.3 }}$ est impuissante à déterminor la mort, qui survient après une nouvelle injection de :3-1e', deux jours après. Essayons si une dose massive, de plus de $10^{\mathrm{c} 3}$ en une fois, est capable de déterminer la mort.

Exp. XLVIII. - 2 cobayes neuf's, l'un mâle, l'autre femelle, pesant l'un 910 grammes, l'autre 876 grammes, recoivent, par la sonde cosophagienne, $20^{\text {e3 }}$ (d'un coup) de suc, identique à celui des expériences ci-dessus.

L.es phénomènes d'intoxication sont tout à fait comparables à ceux observés dans les expériences XLII, XLIII, peut-être un peu plus accusés, mais l'animal se rétablit presque aussi promptement. Le détail de ces observations ne constituerait, après l'exposé des expériences précédentes, que des redites.

Done une dose de $20^{c 3}$ en une fois est aussi impuissante à déterminer la mort qu'une dose de $10^{\mathrm{c} 3}$.

Ces expériences ne peuvent s'expliquer que par une vulnérabilité plus grande du tube digestif, déjà lésé pàr une intoxication antérieure.

Chien.

Exp. XLIX. - Chien, neuf, poids 10 kilos.

10 h. $-33^{\mathrm{c} 3}$ de suc non filtré, dans l'estomac, aucun phénomène appréciable.

$12 \mathrm{~h}$. - $30^{\mathrm{c} 3}$ sont versés, goutte à goutte, dans la gueule; le contact du suc avec la muqueuse buccale détermine des réflexes d'expulsion.

12 h. כ. - Vomissements d'environ $10^{c^{3}}$ de suc, abondamment mélangé de mucus. Le tout est recueilli aussitòt, et réintroduit dans l'estomac; la tolérance gastrique s'établit.

L'animal ne présente qu'une accélération, légère et irrégulière, du rythme respiratoire et cardiaque (phénomène d'ail. leurs de peu d'importance, chez le chien). Le lendemain, l'animal ne présente qu'une très légère diarrhée et un peu d'inappétence.

Exp. L. - 10 h. 30. - Mème chien, $23^{\circ 3}$ de suc dans l'estomac, 
vomissement immédiat d'environ '5³ mélangés à une grande quantité de mucus. Le tout est recueilli et réintroduit dans l'estomac.

11 h. 5. $-\Lambda$ nouveau $25^{3}$.

11 h. 20. - Vomissement de $3^{\mathrm{c} 3}$ environ, avec mucus.

La diarrhée s'établit vers 1 heure, et persiste encore le lendemain, puis rétablissement parfait. Pendant toute la durée de l'expérience, l'animal n'a présenté aucune modification physiologique digne d'être notée.

Exp. LI. - Jeune chiot de 6 semaines. Foids 1 k. 800,10 h. 15. $10^{\mathrm{c}^{3}}$ dans l'estomac. L'après-midi, un peu d'inappétence mais aucun signe net d'intoxication.

Le lendemain $10 \mathrm{~h},-10^{\mathrm{c} 3}$ dans l'estomac, I'après-midi légère diarrhée.

Le lendemain 9 h. $30 .-10^{\text {r3 }}$ dans l'estomac, la diarrhée s'établit, persiste 24 heures. Aucun signe d'intoxication. Rétablissement parfait en apparence.

Il est cependant à noter que, tandis que les chiots de la mème portée croissent d'une façon rapide, celui-ci reste chétil et ne présente, au bout d'un mois, qu'un poids très inférieur à celui de ses frères; il est sujet ¿̀ de fréquentes diarrhées. Il semble bien qu'une susceptibilité particulière de l'intestin succède à l'intoxication par de faibles doses, et persiste à longue échéance.

b) Suc filtré et stéritisé.

Il est inutile d'insister sur le détail des expériences faites avec le suc filtré et stérilisé.

Elles conduisentaux conclusions, précédemment énoncées, relativement au suc d'Arum. 


\section{GHAPITRE V}

\section{MODE D'ACTION ET NATURE DES PRINGIPES TOXIQUES DES ARUM (1)}

En résumé, la foxicité des Aroüdées indigènes, considérahlement exagérée par les auteurs, est néanmoins réelle.

Les expériences, ci-dessus relatées, permettent de conclure assez exactement au mode d'action at at la nature des principes loxirgues de ces plantes. Il importe de bien distinguer deux cas, selon que le toxique pénètre par la voie digeslive, ou directement dans la circulation générale, par voie hypodermique, péritonéale, intrareinense. Nons devons donc résumer le mode d'action des Amm: $1^{\circ}$ sur les voies digestives; $2^{\circ}$ sur les autres systèmes organiques.

Leur action irritante sur les téguments est tellement faible qu'elle est pratiquement négligeable. Lirritation des muqueuses au contact du sue des divers organes des trum est douloureuse, marque par de la congestion; nais nous n'avons jamais pu constater trace d'œdème ni tuméfaction notable.

\section{Action sur le tube digestif.}

Le passage du suc frais dims le tube digestif détermine, ì dose suffisante, des phénomènes de gastro-entérite plus ou noins accusés selon la dose), parfois mortels. Les rongeurs (cobayes et lipinsi se montrent beaucoup plus sensibles à ce toxique que le chien; on peut même dire, sins exagération, qu'il est difficile de détrinner, chez ce dernier animal; une intoxication mortelle par le suc.

(1) Nous entendons ici le terme Arum dans son sens gênéral d'Aroïdées indigènes. Les conclusions ci-dessous s'appliquent aussi bien à l'Arisarum qu'aux Arum proprement dits. 
Le principe auquel le suc doit ses propriétés irritantes est bien un principe chimique. On ne peut allribuer à l'implantalion des riphides dans les muqueuses la part, sinon unique, au moins laplus importante, dans la production des lésions déterminées sur ces muqueuses; la saveur âcre du sue n'est pas due à ces cristaux, car le suc filtré ne perd sensiblement pas de son àcreté.

Mérat et Delexs estimaient déjà que le principe âcre est "sifugace qu'il ne passe pas dans l'eau à la distillation, et que l'alcool ne peut le séparer ) des tisstis qui le renferment. Récemment encore, Gresnofr (d'accord en cela avec lit plupart des auteurs) altribue aux seuls cristaux d'oxalate de chaux la toxiciti des Arum, déniant par là tout pouvoir toxique à la saponine, regardée par SPICA, comme le principe actif.

En réalité, l'âcreté et le pouvoirirrilantreviennent, peut-ètre, pour une très faible part à la saponine, mais surtout à l'alcaloüde; le fait n'a point lieu de nous étonner, sachant les rapports de ce corps avec la conicine de ciguë, dont la saveur est extrêmement âcre.

Un premier point à élucider est la solubilité possible de l'oxalate de chaux en raphides dans les sucs digestifs.

Le seul suc digestif capable de dissoudre ce sel est évidemment le suc gastrique, gràce à son IICr. Expérimentons-le à cet égard.

Le suc, riche en raphides, extrait par expression modérée des parties vertes ou du péricarpe d'Arum, est ver'sé sur un filtre, en quantité suffisante pour obtenir un dépôt abondant, où les aiguilles cristallines se trouvent par milliers.

De petites parcelles de ce dépôt sont examinées au microscope, sur lamelle, et soumises à l'action du suc gastrique artificiel.

L'insolubilité dans ce liquide des raphides est absolue.

Liexpérience est répétée avec des solutions aqueuses d'HCl pur, à titre croissant; il faut un séjour de plusieurs heures, dans HCl pur et fumant, pour obtenir la dissolution des raphides. 
Mêmes essais aree des solutions d'acide liceligur. Con acide pur est impuissant à dissoudre les raphides, mème an boul dre huil jours.

Le sue gastrifue est done lotalement impuissint it dissoudre les aiguilles cristallines d'oxalate de chaux.

Cette expérience, tentée avec les Arum, a été répélée avec le même résultat sur les raphides de diverses Monocolylédones fen particulier de T'amus rommunis), que l'on peut supposer capables de déterminer sur les muqueuses une irritation plus ou moius violente, du fait de l'implantation de ces milliers de microseopiques aigruilles. D'une manière générale, on est donc autorisé à conclure que les raphides d'oxalate dechaux, contenus dans les tissus vigelaux, traversent sans être dissous le tube digestif.

Cette conclusion est d'ailleurs confirmée par l'examen microscopique du contenu de l'intestin, quelques heures après l’ingestion d'un liquide riche en raphides.

Ces cristaux se retrouvent intacts dans le contenu intestinal.

Les organes ou le suc, privés, par la chaleur ou l'éraporation, de l'alcaloïde, perdent toute propriété irritante.

Relatons ici un certain nombre de faits, capables d'élucider le rôle des raphides dans la production des lésions observées sur le tube digestif.

Les raphides sont-ils susceptibles de s'implanter sur les muqueuses, et d'y déterminer une foule de minuscules lésions élémentaires?

La question est assez difficile à trancher par l'examen direct de la muqueuse digestive; mais une expérience indirecte est assez probante.

Une parcelle du dépôt cristallin, laissé par la filtration d'un liquide riche en raphides, est déposé sur la cornée d'un cobaye ou d'un lapin, les paupières fermées avec le doight, et une friction circulaire, légère el de quelques minutes, exercée sur le globe de l'oeil. L'expérience n'est pas douloureuse, l'ail n'est ni larmoyant, ni injecté. Quelques heures s'écoulent, la cornée voit alors sa sensibilité très diminuée, à mesure que son opacité se déclare, sous la 
forme de pelites taches laiteuses, très visibles, lorsrgu'on examine l'wil à jour frisant. Le lendemain, une large tache oparque occupe toute la face extérieure de la cornée, et une injection vive de toute la sclérotique apparait. La cornée ainsi lésée est incapable de reprendre sa transparence primitive. Les lésions cornéennes évoluent avec une grande rapidité, surtout au cas où l'on dépose sur l'uil des raphides, empruntés ì un liquide exposé ì l’air et déjat en fermentation; dans ce cas, le traumatisme cornéen se complique d'infection microbienne; chaque raphide joue le róle d'une minuscule aiguille d'inoculation.

Les mêmes faits se passent-ils à la surface des muqueuses? Evidemment oui, mais avec une intensité plus faible. Nombre de raphides doivent être entrainés, enrobés dans le mucus, sans trouver à s'implanter dans les cellules épithéliales.

Les expériences déjà relatées (v. p. 97) sur l'innocuitê d'un liquide tenant en suspension des raphides seuls montrent que les désordres, dus à l'ingestion d'un suc riche en raphides, ne peuvent être altribués à la simple action mécanique de ceux-ci.

Il importe d'avoir bien présent à l'esprit ce fait, que nombre de plantes, susceptibles d'être consommées par les herbivores ou par l'homme, sans que le moindre inconvénient résulte jamais de leur emploi, sont cependant fort riches en raphides d'oxalate de chaux, par ex. : Vigne, Enotherct.

Il est facile de comprendre que, si les raphides sont impuissants à déterminer seuls des lésions appréciables des muqueuses, leur présence dans un liquide renfermant un corps irritant et toxique, pourrasingulièrement faciliter l'action de ce dernier, et son passage dans la circulation générale. Comme confirmation de cette hypothèse, rappelons que la toxieité du suc, débarrassé par simple filtration de ses raphides, est un peu plus faible que celle du suc brut. Les principes toxiques solubles sont cependant, à eux seuls, capables de déterminer la mort et les lésions caractéristiques de l'intoxication. 
La présence des raphides dans le suc n'est pas nécessaire pour déterminer des symptòmes graves et même la mort. C'est donc aux principes solubles (alcaloïdes ou saponine) (qu'il faul rapporter le pouvoir irritant exercé sur le tube digestif, mais l'action mécanique des raphides, contribur très vatisemblablement an developpement des lésions.

Est-ce à la saponine ou à l'alcaloüde que l'on doit rapporter la toxicité ou pouroir irritant? Nous ne pouvons encore donner une réponse absolument catégorique à celte question, car noụs n'avons pu isoler la saponine d'Arum en quantité suffisànte pour déterminer ses propriétés physiologiques.

Administrée par voie stomacale, la saponine type est extrêmement irritante pour le tube digestif, elle est violemment émétique, enflamme la mucrueuse avec laquelle elle se trouve en contact, détermine la congestion de l'intestin, et souvent une entérite hémorrhagique.

Mais il suffit de réfléchir à la faible quantité de saponine que renferme une dose mortelle de suc d'Arum, pour reconnaitre l'impossibilité d'attribuer à ce corps toute l'activité dont jouit ce suc. Lal présence simultance d'un autre toxicque alratoïde arroitrait-elle l'activité de la saponine? C'est. une question à résoudre ultérieurement.

Une expèrience simple prouve, d'ailleurs, que les organes d'Arum les plus riches en saponine (tubercules au repos), privés d'alcaloïde (par ébullition ou dessiccation), peurent eitre ingérés impunément à fortes doses.

Nous avons vu l'alealoïde provoquer la mort par son introduction dans le tube digestif. Rappelons que l'introduction du suc ou de la conicine par une autre voie détermine une congestion nette des voies digestives. C'est donc à l'alcaloïde, et à son contact direct avec la muqueuse, que doivent être attribués surtout les désordres observés.

Quel est le mode d'action du suc d'Arum sur les voies digestives? Il agit à la manière des irritants en général, détermine l'hyper- 
sécrétion des glandes, la congestion de la muqueuse, l'exsudation d'un liquide séreux-sanguinolent, la desquamation de l'épithélium.

Nous n'avons pas essayé de déposer directement le suc sur la surface externe de l'intestin pour deux raisons:

$I^{\circ}$ Il ne peut agir sur les terminaisons nerveuses intestinales qu'après avoir traversé les couches musculaires; les éléments anatomiques qu'il peut impressionner se présentent, dans une disposition inverse de celle où ils se présentent lorsque le toxique est déposé sur la face interne.

20 Il agira forcément sur le péritoine, pourra être absorbé directement par cette séreuse et produire des actions réflexes ayant le péritoine pour point de départ, ce qui ne se produit pas dans le cas d'injection du toxique.

Une remarque digne d'intérêt est la suivante :

Une même dose de suc, portée à l'aide de la sonde dans le tube digestif, produit des effets bien plus marqués, si la sonde, molle, flexible, est susceptible de se courber au contact du fond de l'estomac et de faire affleurer son bec au voisinage du pylore.

Une faibledose de suc $\left(3^{\mathrm{c} 3}\right)$, ainsi déposée au contact du sphincter pylorique, le force facilement si la pression exercée par la poire insuflatrice est suffisante; le suc arrive ainsi directement dans l'intestin grèle. Dans ces conditions spéciales, $3^{\mathrm{c} 3}$ de suc produisent des effets plus énergiques que $10^{c^{\prime 3}}$ du mème suc, simplement déposés dans l'estomac.

Une autre expérience parle dans le mème sens.

Sur un cobaye, fixé sur la planchette d'expérience, on pratique une petite incision à la paroi abdominale, juste suffisante pour atteindre une anse intestinale, et y implanter une aiguille de seringue de Praraz. Cette implantation faite, on laisse reposer l'animal, en rapprochant les lèvres de l'incision, et, au bout d'un quart d'heure, on injecte arec l'aiguille implintée dans l'intestin), un peu de suc dans une anse intestinale.

On peut ainsi voir que $3,5^{\mathrm{e} 3}$ de suc frais (de parties vertes) d'Arum, déposés directement dans une anse intestinale, excitent plu- 
tôt ses mouvements péristalliques, déterminent son injection vasculaire, et la transsudation d'un liquide séro-sanguinolent.

Il nous paraîtrait prématuré de donner une explication de ces faits. Se raltache-t-elle à une absorption plus rapide do foxique par la muqueuse intestinale que par la muqueuse stomarale, on a une irritation plus facile et plus vive des terminaisons nerveuses?

Un tube digestif, dont la muqueuse est en partie desquamée, absorbera les toxiques solubles aroc une rapidité infiniment plus grande, que ne le ferait une muqueuse saine.

De plus, l'irritation mécanique raphides et chimique principe âcre) des terminaisons nerveuses sera bien plus intense dans un intestin lésé.

Sil'on admet que, de la vulnérabilité plus ou moins grande du tube digestif, dépend la gravité de l'inloxication, on sera amené à cette conclusion qu'une mime juantite de plante, egalement riche en principes toxiques, produira, chez deux sujets differents, une intoxication dont la gravité et même certains symptômes pourront être très différents.

Étant donnée la toxicité variable d'un même suc, ingéré par la roie digestive, il serait assez illusoire de prétendre déterminer son coefficient de toxicité, lorsqu'il est administré par cette voie.

Cette toxicité pourra, d'ailleurs, ètre variable, suivant l'éporfue de végétation de la plante à laquelle ce suc est emprunté.

Il est fort difficile de préciser sur quels éléments de la muqueuse agit de préférence le toxique.

L'action directe sur l'épithélium n'est pas douteuse. C'est sur les cellules épitheiliales que soimplantent d'aburd les raphides. Cette lésion mécanique, combinée à la toxicité des principes solubles. provoque la chute de l'épithélium. L'épilheilium intestinal se détache comme dans d'autres intoxications (choléra, arsenic, muscarine).

L'excitation directe des éléments sécréleurs provoque leur hypersécrétion, qui est encore facilitée par la congestion ultérieure de la muqueuse. 


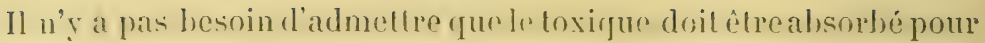
agir sur les éléments nerreux de l'intestin. Il suffit qu'il imbibe la cellule épithéliale, pour que les terminaisons nerveuses, à l'intérieur du protoplasme de ces cellules, soient irritées, d'ou point de dipart du riflexe. Dailleur's, lat wute de lippithélium met en contact direct les fibrilles nerveuses arec le liquide irritant.

La constatation d'un péristaltisme assez intense, aussitôt, ou peu de temps après la mort, ne prouve pas cu'il est contemporain des derniers temps de la vie.

L'intestin de l'animal expirant peut être au repos complet, et le péristaltisme s'accélérer peu à peu par paralysio des centres supérieurs, paralỵsie résultant de l'arrêt de la circulation mème au cas où l'on cherche à éviter, autant que possible, l'action irritante de l'air).

La circulation pendant le coma étant très ralentie, les centres modérateurs sont en état d'asphyxie.

Le péristaltisme peut donc ètre d'origine asphyxique, et ne pas ètre primitif, dù au toxique et coïncidant pendant la vie avec l'action de ce toxique.

La constatation d'un péristaltisme assez intense chez un sujet mort dans le coma n'est donc qu'insuffisamment instructive.

Mais nous avons dit plus haut que l'injection directe du toxique, dans une anse intestinale, excite plutiot ses motrements péristalliques, en mème temps qu'elle y détermine de la congestion.

Le suc d'Arum ne diminue pas l'excitabilité d u plexus myentérique, puisqu'il ne ralentit pas les mourements péristaltiques. Il n'excite pas non plus les fibres d'arrêt de ces mouvements.

On ne peut donc admettre que, par voie réflexe, le nerf splanchnique est irrité, puisque l'on sait que son excitation détermine l'arrêt des mouvements péristaltiques (Guvon et Courtane) (1).

Excite-l-il, au contraire, l'appareil moteur de l'intestin (fibres motrices du splanchnique) (comme la nicotine, la muscarine, la caféine) ? La chose semble douteuse, car il n'accélère pas l'évacuation de l'intestin, et ne produit pas de selles licquides peu de temps

(1) Guxon et Courtade. Act. du sympathique sur la contraction de l'intestin, (Soc. Biol., 5 décembre 4896.) 
après son ingestion. (Lal diarrhie ne s'établil que lardivemrul, of n'est jamais très abondante.)

Il semble plutôt que ce soit un excitant direct de l'intestin, capable de provoruer une latussudation aqueuse des vaisseaux inleslinaux.

L'excitation primitive doil porter sur les terminatisms nerrenses et les plexus nerveux.

Gelteconclusion ne s'appliquerait peul-ètre qu'au début de l’irritation intestinale, si l'on admel, ares: S. MA rerel r. Basch, qur', dans un intestin congestionné, lat veinosite du sang paralyserail les fibres d'arrèt du splanchnique (O. NAsse). L'excitation du splanchnique détermine, au contraire, des mouvements péristaltiques.

L'hypothèse d'une paralysie des fibres motrices du splanchnique parait cependant plus conforme aux fails, car la paralysie fibres vaso-motrices intestinales est incont estable; d'où dilitalion paralytique des vaisseaux du tube digestif.

Mais, dans cette intoxication, la raso-dilatation paralytirgue do la circulation abdominale n'est pas assez intense pour déterminer l'anémie des autres territoires vasculaires, et amener la mort par l'état ersangue des centres nerveux. A l'autopsie on troure, en effel, constamment, ces centres congestionnés.

On sait que l'excitation des nerfs splanchniques (par excitation directe portée sur la moelle lombaire) provoque l'hyperhémie do la muqueuse intestinale, parfois l’hémorrhagie el laspect disentérique de l'intestin (1).

La transsudation d'un liquide séreux, phénomène caractéristique et constant, rappelle, de tous points, la sécrétion paralytique qui s'observe après la section des nerfs du mésentère. La suppression, par suite de laction du toxique, des gimglions sympathiques doil agir comme la section des nerfs.

Il ne faudrait cependant pas altribuer la mort it une diarrhéc

(1) 1874, 1875, 1876. - Lauder Brunton ('T.) and Pre-Smitu. Intestinal Secretion and Movement (British-Association Reports).

1889. - Lauder Bruxtos (T.). Tr. de Pharmacologie (trad. franc., p. ${ }^{439)}$. 
mortelle résultant de la suppression physiologique des ganglions sympathiques.

Les fibres sensitives du splanchnique sont certitinement soumises à unc excitation violente, d’où une série de reflexes, les uns sur l'intestiu lui-mime, les autres sur la respiration et la circulalion.

L'action du toxique sur le tube digestif n'est pas seulement directe, alle est aussi indirecte, par suite des réflexes portant sur la circulation et l'innervation de l'intestin.

In premior reflexe peut se produire par les filores excilatrices que le pneumogastrique fournit à l'intestin.

\section{Action sur la respiration et le système nerveux.}

Les rétlexes respiratoires provoquent des modifications du rythme el de l'amplitude des mourements respiratoires, déjà indiqués dans la relation des expériences.

Une première phase d'accélération de ces mouvements est conlemporaine du début de l'intoxication, puis le rythme se ralentit, ct devient particulièrement intéressant à étudier à la période de l'intoxication.

Les tracés ci-joints fig. ¿8 expériences parr intoxication mortelle du cobaye par le suc d'Arisarum) montrent les modifications du rythme et surtout du type respiratoire, a mesure que l’intoxication s'accentue. Ils prêtent aux remarques suivantes :

10 h. อ̃ (I). Début de l'expérience, tracé normal.

11 h. 7 (II). Le nombre de mouvements respiratoires par minute rst normal, leur amplitude est légèrement augmentée; à intervalles réguliers survient une inspiration profonde, forcée, prolongée (le plateau l'indique), suivie d'une expiration moins brusque qu'à l'état normal.

11 h. 11 (III, IV . Léger ralentissement du rythme, la respiration périodique s'établit.

11 h. 13 (V). Court accès de polypnée, accentuation des inspira- 

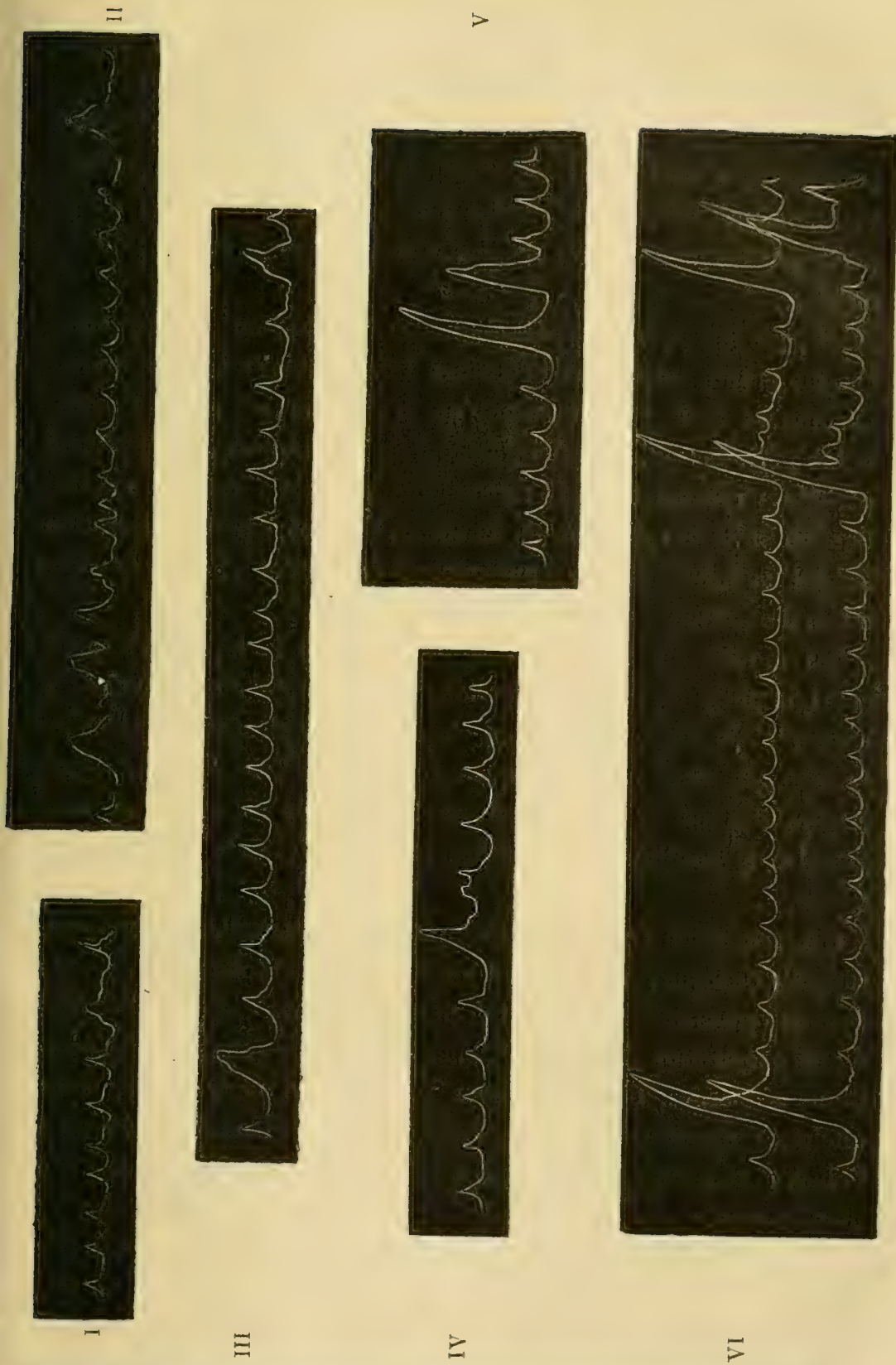

Fig. 58. Modifications des mouvements respiratoires dans l'intoxication mortelle par le suc d'Arisarum (tracés recueillis avec le cardiographe à double ampoule pour cobaye). Expér. XLVI. (Photog. directe, sans réduct.). 
tions forcées. Chaque inspiration forcée est brève, suivie par instants d'une seconde inspiration forcée, mais semi-avortée.

12 h. VI. Accés de polypnée, mourements respiraloires de faible amplitude; à intervalles réguliers, inspirations forcées, brèves. Par moments, tétanos partiel des muscles inspirateurs, qui n'empêche pas les mouvements respiratoires de se produire rythmiquement.

Lorsque la respiration périodique est franchement établie, entre chaque grande inspiration se trouve intercalée une période, qui se décompose assez régulièrement en trois courtes périodes : première période : rythme ralenti (relour au rythme normal); deuxième période : rythme accéléré; troisième période : rythme ralenti. Chaque forte inspiration, ou chaque petite période d'accélération semble suffire à rétablir pour quelques secondes l'hématose normale, d'où relour au rythme normal.

Ces modifications peuvent se résumer de la façon suivante : accès de polypnée (avec diminution de l'amplitude des mouvements respiratoires, et, à intervalles réguliers, inspirations forcées et brèves) alternant avec quelques courtes périodes où le rythme respiratoire est ralenti. Finalement arythmie, diminution progressive et continue du nombre et de l'amplitude des mouvements.

L'intercalation de grandes inspirations à intervalles rythmiques, rappelle quelque peu la respiration de Cheynes-Stokes, si fréquente dans diverses intoxications, ou, plus exactement, elle est l'indice d'une respiration franchement périodique. La respiralion périodique s'observe, d'ailleurs, avec une netteté particulière, dans quelques intoxications. Par exemple, chez les chiens chloralisés, on l'observe très nettement, quant aux contractions respiratoires du diaphragme.

De temps à autre, suivant une périodicité régulière, une sorte de soupir, un mouvement respiratoire plus ample que les autres, interrompt la régularité des mouvements respiratoires à faible amplitude. C'est l'exagération de la respiration périodique, que l'on peul constater, avec plus ou moins de netteté, suivant les sujets 
et les curconslances, sur les sujets normatux pendant l'iblat de sommeil (Mosso).

A mesure que les inspirations devienment plus fréquentes, leur amplitude diminue, la rentilation se trouve finalement diminure.

Mais, comme on sait que de petites inspirations renouvellent parfaitement lair des alvéoles pulmonateses, on ne pent pats allribuer uniquement la grande inspiration periodique at l'amoxhémie, déterminée par un état asphyxique.

Se raltachant à l'hypothèse, proposée par Frédénice et IIÉRING (pour expliquer la respiration périodique, al l'étal normal), on pourrait supposer que les grandes inspirations se produisent pour assurer une sorte de régulation de la pression artérielle.

Dans l'état d'intoxication, les petits vaisseaux accentueraient leur rôle de cœur périphérique, en exagerant, à intervalles régruliers, leurs contractions périodiques, et provorquant ainsi des oscillations lentes de la pression artérielle, au moins sur certains territoires.

L'intérêt de cette remarque, hypothétique assurément, mais digne, ce nous semble, d'être présentée, seraít de meltre en relief un phénomène nerveux périodique, d'intensité notable, au cours de l'intoxication que nous étudions.

Il ne me semble pas nécessaire d'admettre que le centre respiratoire est directement influencé par le toxique (conicine) entrainé dans la circulation.

Les changements respiratoires observés semblent être dus à ce que le centre recoit des excitations périphériques (partant du tube digestif) anormales; l'état d'anoxhémie du sang doit aussi modifier son excitabilité.

Il n'est pas nécessaire d'admettre que c'est l'absorption des principes solubles, dans l'intestin, qui détermine les modifications observées du côté du poumon et du cour. L'irritation et les lésions de l'intestin suffisent à expliquer le retentissement réflexe sur ces organes.

Le rythme spécial de la respiration permet à lui seul de conclure que le cœur ne s'arrête pas avant la respiration. 
lin effet, dans toutes les intoxications oủ le cơur est arrêté avant la respiration, on observe non pas le rythme asphyxique, mais le rythme agonique. Ce rythme est caractérisé par les phénomènes suivants :

Lors de son établissement, on observe de petites respirations fréquentes (derniers soupirs), qui s'affaiblissent, puis cessent; une longue pause survient, puis tout à coup apparait une grande respiration très profonde, suivie de deux ou trois autres; nouveau silence, et la même série de modifications se reproduit deux, trois ou quatre fois, jusqu'ì ce que finalement la respiration cesse.

A la période d'intoxication confirmée par les Aroïdées, les symptômes sont bien ceux de l'asphyxie lente.

Pendant une première périnde d'accélération respiratoire, l'animal est haletant, agité, cette première période est relativement courte; pendant la $2^{\circ}$ et dernière période, beaucoup plus longue, et qui aboutit au coma, la respiration est au contraire plus ample, plus calme; l'angoisse, les efforts d'inspiration pour humer l'air (narines et bouches ouvertes), l'hypothermie constante suffisent à dénoter l'état asphyxique. La mort survient par l'arrêt des mouvements respiratoires, puis du cœur.

Or, dans l'asphyxie, il y a d'abord accélération, puis ralentissement, puis arrêt des mouvements respiratoires.

Le cœur s'accélère d'abord, et sa force augmente, puis ses mouvements se ralentissent, jusqu'ì atteindre le chiffre de 3 à 4 par minute, puis ils s'accélèrent, ce qui est le signal d'une mort prochaine (1).

Il serait certainement exagéré de dire que les Aroïdées tuent par asphyxie. L'asphyxie est la terminaison des intoxications par un grand nombre de substances, dont l'action physiologique est essentiellement différente.

Comme l'organisme doil finir par succomber à une altération fonctionnelle quelconque, c'est très souvent par l'asphyxie que se termine la scène. Dans l'état de dépression de toutes les fonctions

(1) 1895. - Richet (Crr.). La mort du cœur dans l'asphyzie. (Arch. de physiol.). 
où se trouve l'agonisant, on ne peut pas direque la vraic cause de la mort soit l'asphyxio: c'est seulement le mode de la mort.

Ingérés par la voie digestive, les Arum tuent en déterminant une gastro-entérite aiguë, avec retentissement réflexe sur la respiration rt la circulation. La mort survient finalement dans le coma et par le mode asphyxique. Le retentissement réflexe sur lo système nerveux central (convulsions, délire), signale dans les cás l’inloxication chez l'homme, ne s'observe pas dans l'intoxication expérimentale chez les animaux.

Les lésions observées à l'autopsie sont celles de la gastro-entérite et de l'asphyxie.

La toxicité revient aux principes solubles, surtout ì l'alcaloöde ; les lésions par les raphides ne jouent que le rôle de causes adjuvantes.

L'intoxication avec les Arum, par voie hypodermique ou péritonéale, ne relève que de leurs principes solubles (puisque les raphides sont éliminés des liquides d'injection).

\section{Toxicité de la saponine d'Arum.}

Les expériences avec l'extrait alcoolique (V.p. 103) (qui contient lil presque totalité de la saponine et une quantité insignifiante d’alcaloïde) révèlent une réelle analogie entre les symptômes déterminés par l'injection de cet extrait, et ceux que l'on observe après l'injection des saponines toxiques.

Une des propriétés les plus caractéristiques de la saponine est de déterminer, par injections sous-cutanées, une anesthésie locale. Cette propriété appartient aussi à l'extrait d'Arum. Il jouit aussi des propriétés phlogogènes de la saponine. Cependant la saponine, mise en contact avec les nerfs et les muscles striés, en abolit instantanément l'irritabilité, ce qui ne se produit pas avec l'extrait d'Arum.

Selon Scumedeberg, la saponine agit sur la pointe du cocur 
de la grenouille, en abrégeant la durée de la systole cardiaque, aflidiblissant graduellement l'énersio des pulsations ventriculaires, arrêtant finalement le cœur en diastole.

Il suffit de se reporter aux expériences (V. p. 105) pour voir que la saponine d'Arum n'agit pas ainsi sur le cœur. Des doses relativement considérables d'exlrait sont nécessaires pour déterminer une intoxication mortelle. Si done li toxicité de la saponine d'Armm semble incontestable, elle est cependant plutôt faible.

Par injection hypodermique, les sapotoxines déterminent surtout des phénomines du coté de la moelle; il semble que l’on puisse, dans cette intoxication, distinguer deux phases, mal mises en lumière par les auteurs qui l'ont étudiée: $1^{\circ}$ phase d'excitation, d'où agritation, tremblements musculaires, respiration accélérée, pouls plein et fréquent; $2^{\circ}$ phase de dépression, puis de coma, où la sensibilité et la motilité disparaissent, la respiration devient lente, puis s'éteint en même temps que le cœur s'arrête. A l'autopsie, on ne constate gù̀re qu'une injection du côté des méninges. Le tableau de cette intoxication se rapproche donc, sur bien des points, de celui de l'intoxication par des doses élevées d'extrait d'Arum.

N'ayant pu rechercher l'action de l'extrait sur les éléments figurés du sang, il nous est impossible de les comparer à ce point de vue à la saponine qui jouit, on le sait, de propriétés dissolvantes énergiques vis-à-vis des hématies.

L'extrait d'Arum ne modifie qu'à peine la contraction cardiaque chez la grenouille et le lapin.

Il est donc logique de conclure que la toxicité de l'extrait revient à la saponine qu'il contient; mais les organes et le suc ne renferment que des quantités beaucoup trop faibles de saponine, pour pouvoir faire jouer à cette substance un rôle prédominant dans l'intoxication. Même isolée, elle jouit de propriétés physiologiques bien moins accusées que les sapotoxines types.

La toxicité appartient surtout à l'alcalö̈de. Si les Amm perdent par dessiccation et par ébullition tout pouroir toxique, c'est qu'ils sont privés de conicine par ces manipulations. 


\section{Toxicité de l'alcaloïde.}

L'expérimentation directe avec la conicine d'Arum confirme cette opinion. Chez les animaux intoxiqués par cette base, un des phénomènes les plus nets est la paralysie immédiate du membre injecté; c'est là un des catractères essenticls de l'intoxication par la conicine de ciguë.

L'anesthésic au point d'inoculation, le pouvoir curarisant, l'arrêt de la respiration, tandis que le ceur continue ia battre, les convulsions, sont autant de traits communs aux intoxirations par les deux substances.

Les lésions constatées à l'autopsie des animaux qui succombent à l'intoxication par l'alcaloïde d'Arum, sont bien celles de l'intoxication par la conicine (sang noir et fluide, taches pétéchiales, congestion des méninges et des viscères).

Toutes ces considérations nous amènent it conclure que les Arum, ingérés par voie digestive, tuent par la gastro-entérite aiguë qu'ils déterminent, d'où réflexes pulmonaires et cardiaques aboutissant au coma; cette action purement réflexe doit ètre aidée par l'absorption de la conicine.

La conicine d'Arum, portée directement dans la circulation générale, agit (bien qu'arec moins d'énergie) comme la conicine type, et détermine la mort par paralysie musculaire et action sur le bulbé. 


\section{CHAPITRE VI}

\section{L'INTOXICA TION SPONTANÉE PAR LES ARUM}

Nous ne possédons qu'un nombre relativement très restreint de relations complètes de cas d'intoxication par les Arum. Tous les auteurs, qui parlent de leur's propriétés toxiques, semblent plutôt s'inspirer des opinions émises par leurs devanciers que relater leurs observations propres. Pour chercher ì établir le tableau symptomatique de l'intoxication spontanée par ces plantes, il y a tout intérêt à rapprocher les résultats des observations faites sur les animaux domestiques, de ceux fournis par les observations ayant spécialement trait ì l'espèce humaine.

\section{Intoxication par les tubercules.}

Au printemps, les sangliers retournent dans certaines forêts toutes les places où s'épanouissent les Arum, afin vraisemblablement d'en trouver les tubercules (nous avons pu constater nousmêmes le fait.)

Le rhizome d'Arum est parfois déterré, à la sortie de l'hiver, par le pore, qui le consomme, mais jamais en quantité suffisante pour s'intoxiquer mortellement. L'animal est sans doute rebuté rapidement par la brùlure que le passage des fragments de tubercules broyés détermine dans la gueule et l'arrière-gorge.

La gueule et la langue du porc qui a mâché quelques tubercules rougissent et se tuméfient, la salive coule, bientôt la déglutition devient difficile en raison de l'inflammation de l'arrière-bouche. Connevix, à qui nous empruntons ces détails, semble avoir été témoin de l'intoxication des bestiaux par l'Arum, et décrit, comme suit, ses symptômes.

Introduit en petites quantités, dans le tube digestif (comme c'est toujours le cas dans les empoisonnements spontanés des bestiaux, 
l'Arum provoque de vives douleurs intestinales, de l'agitation, un peu de contraclion musculaire des membres, du balancoment de la tête, une superpurgation avec épreintes. Le ventre reste douloureux, et l'appétit faible pendant quelques jours.

OrFILA a fait périr des chiens en leur faisant ingérel le rhizone frais d'Arum. La mort survenait de 20 a 36 heures après l'ingestion, par suite de l'irritation gastro-intestinale.

Vient-on à porter à la bouche un rhizome frais d'Arum, qu'à peine entamé avec les dents, il détermine une bruture immédiate, quifait vite lìcher prise, et peut persister, loul en décroissant peu à peu d'intensité, pendant plusieurs heures. Yous avons pu le constater sur nous-meme, et sur diverses personnes qui, atu cours d'herborisations, voulaient vérifier par elles-mèmes l'opinion courante, relativement ḋ l'àcreté extrème des tubercules d'Arum. Cru, ce tubercule ne parait devoir itre consomme jamais en quantité suffisante pour déterminer autre chose qu'une irritation forte, mais passagère, de la muqueuse buccale.

Il existe cependant une relation d'intoxication spontance par tubercules d'Arum, due à CANGeLla.

\section{Cas d'intoxication chez l'homme par tubercule d'Arum.}

I. (CANGella.) - Un jeune enfant de 3 ans ayant mastiqué, le 20 avril 1860 à 2 heures de l'après-midi,quelques racines d'Anum, se plaignit aussitót de sensation de brúlure sur les lèvres et sur la bouche. $3 \mathrm{~h}$. après il était dans un profond état de stupeur, auquel succéda une réaction fébrile intense. A 8 h., Cangella trouva le petit malade dans une prostration complète, incapable de parler et portant souvent ses mains à sa bouche et à sa gorge, en poussant par moments un cri rauque comme s'il étouffait. Cangella constata que l'action caustique de la plinte s'etendait aux lèrres.au palais, à la langue, aux amygdales et au pharynx, autant qu'il put voir; mais la douleur ressentie, en appuyant sur l'estomac, lui montra que l'enĩant avait avalé du jus, et que l'action caustique s'étendait plus loin. N'ayant aucun moyen sous la mitin de produire l'évacuation du poison, il administra du sel ordinaire, mais la déglutition était impossible; la contraction était telle qu'on ne pouvait introduire une sonde dans l'œsophage. Les révulsifs les plus énergiques furent employés en vain, et la mort arriva à $11 \mathrm{~h}$. au milieu du délire et de l'asphyxie.

Employé après cuisson, soit comme abortif, soit à titre d'aliment, 
le tubercule d'Arum pris en quantité molerée semble, d'après nos essais voir pagre 89, ne deroir déterminer ancun accident. Au cas nù il serait employé d'une facon suivie, comme plante alimentaire, il se pourrait qu'il déterminât, à la longue, comme d’autres Arö̈dées, desphénomines d'intoxication lente, dont l'élude ressort bien plutôt de l'hygiène que de la toxicologie proprement dite.

Par contre, le rhizome d'Arisorum v'nlgare serait susceplible d'ùtre assez fréquemment pris, dans nos provinces méridionales, pour la racine de radis ou de navels de petite dimension.

Certains auteurs affirment que, de ce fait, des intoxications par celte plante ne seraient point rares chez les campagnards des régions où l'Arisurum pousse en abondance. Mais, à notre connaissance, aucun cas d'intoxication n'a été jusqu'ici relaté avec précision.

La confusion ne peut évidemment s'établir entre le navet et les parties souterraines de l'Ariscrum qu'au cas, assez rare d'ailleurs (voir page 49 et pl. II et III), où ce dernier aurait ses organes souterrains réduits à une unique masse tuberculeuse ovoïde. Pendant toute la période de végétation, la forme si caractéristique les organes aériens doit suffire à empếcher toute méprise : ce n'est que dans la période de repos que le tubercule, mis à nu en remuant la terre, pourrait ì la rigueur prèter à confusion. Sa saveur, tout aussi âcre que celle des Arm, doit d'ailleurs vite rebuter. Cuit, il parait tout aussi inoffensif que les premiers, et peul ainsi être employé impunément, d'une façon peu courante d'ailleurs, semble-t-il, dans certaines campagnes méridionales, comme aliment.

L'opinion de Bulliard, que cette plante ne doit pas être susceptible de déterminer d'intoxication spontanée, semble, malgré l'opinion contraire des auteurs, assez justifiée.

\section{Intoxication par les feuilles.}

Les feuilles d'Arum, lorsqu'on les froisse et surtout Ies broie, exhalent une odeur vireuse. Cette odeur, jointe à leur saveur brûlante, lus fait, en général, dédaigner par tous les animaux domestiques. On ne connaît pas de cas d'intoxication, par ingestion de 
feuilles d'Arem, chez nos herbivores. On suppose, ceprodint mais

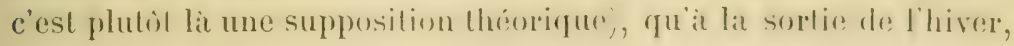
les bestiaux, soumis pendant plusieurs mois au régime du sec, finissent par l'acepeler, mais sans en prenelre un duantilé suftisante pour succomber.

On a vu des cobayes plutôt se laisser mourir de faim que de tou(cher aux femilles d'Alum (Connevix). Nos expériences it cet rigard parlent dius le mème sens; mème après un joùne prohongé, lapins et cobayes refusent même d'entamer les pousses verte des Arum, elles sont rejetées par tous les herbivores : bœuf, mouton, cheval et chèvre, auxquels on les présente.

Ce doit-être l'odeur spéciale de la plante qui la protège contre la dent des herbivores, plus encore que sa saveur brüante, el les raphides dont tous ses membres sont remplis.

Dans la campagne, les feuilles d'Arum ne portent jamais trace de la morsure des escargots ou des limaces, si avides pourtant, au premicrs beaux jours, de toutes les pousses fraîches.

La feuille d'Arum ne sert de nourriture à aucun insecte phyllophage. Nous ne l'avons vue infestée que par un Aphis, et par un Acarien.

Bulliard déclare queles feuilles d'Arum peuventètre, par mégarde, màchées et avalées, parce qu'elles sont insipides !) et qu'ainsi, il arrive fréquemment que l'on confonde la variété d'Arum maculatum, privée de taches noires, arec les feuilles d'Oseille sauvage.

Telle est aussi l'opinion de Mérat el Delens; cette opinion est d'ailleurs appuyée par la relation d'un certain nombre de cas d’intoxication spontanée, surrenus chez des enfants, à la suite de l'ingestion de feuilles d'Arum. Voici le résumé de ces cas :

\section{Cas d'intoxication chez l'homme par feuilles d'Arum marulatum.}

II. (Bullard.) - En 1769, dans la forêt d'Arc, trois enfants de búcheron mangèrent les feuilles de cette plante; il leur prit des convulsions horribles; on tarda trop à leur apporter des secours; il fut impossible de rien faire avaler aux deux plus jeunes : on les saigna sans succès, on leur donna des lavements qui ne produisirent aucun effet; ils périrent l'un au bout de douze heures, et l'autre au bout de seize. Le $3^{\mathrm{c}}$ fut sauvé 
par les soinsde. II. Artus, jeune médecin dont les talents nous sont connus. Cet enfant avait sans doute moins avalé des feuilles de cette plante que les deux autres; il paraissait aussi d'une constitution plus robuste; [il pouvait encore avaler, quoique avec beaucoup de peine, parce que sa langue était tellement tuméfiée qu'elle remplissait toute la capacité de sa bouche; mais la déglutition devint plus libre quand il fut soigné; on lui fit boire du lait, de l'eau tiède, et surtout beaucoup d'huile d'olive; on lui donna aussi plusieurs lavements avec de l'huile d'olive, il lui survint une diarrhée qui le sauva; il fut assez bien rétabli en peüde temps, mais il a toujours conservé une très grande maigreur.

III. (Fraser). - Jolın Call, âgé de six ans, fut trouvé par sa mère, trois heures avant que je le vis, couché sur le bord de la route, à plat ventre, "dans une convulsion," me dit-elle, qui, ne cessant pas, l'obligeait ì m'amener l'enfant.

Je constatai une contraction spasmodique de tous les muscles du corps, une écume sanglante à la bouche, les mains fermées, les pupilles dilatées et les yeux en haut, et la langue mordue; il se faisait aussi dans la gorge un bruit choquant, semblable à celui qu'on entend chez les épileptiques; les lèvres et la face étaient très pâles, le cœur très faible, le pouls petit et intermittent.

Les secousses convulsives duraient chacune quelques minutes, et cessaient pour un temps de même durée; lorsqu'elles cessaient, les muscles de la face étaient agités d'un tremblement particulier. Je trouvai qu'il était impossible de faire avaler quoi que ce soit, la bouche du petit patient étant fermée fortement et serrée.

J'arrivai cependant, après quelque temps, ì donner une solution de tartre stibié, et j'approchai une forte solution d'ammoniaque sur les narines. La dose d'antimoine absorbée n'était pas assez forte pour produire le vomissement, mais il y eut bientôt après deux ou trois selles d'une horrible fétidité et, à partir de ce moment, les spasmes furent courts, et beaucoup moins violents. Tout ceci avait demandé $1 \mathrm{~h} .1 / 2$, pendant laquelle je mis aussi des sinapismes sur la poitrine. Alors, les pupilles commencèrent à montrer que l'œil était sensible à la lumière, et l'enfant. commença à sursauter comme le font les enfants qui ont peur d'un coup' coup de fusil ou du diable. - Peu après il put avaler, quand on lui introduisait une pleine cuillerée à thé de liquide dans la bouche; puis je lui ordonnai une tasse de café bouillant qu'il prit avec beaucoup de facilité.

Les sinapismes furent appliqués sur la poitrine et la nuque, et je lui donnai tard, dans la soirée, un peu de calomel et de jalap. $\Lambda 11$ heures du soir, il était encore insensible quand on lui parlait, mais quand on lui présentait une boisson, il l'avalai i, avec quelque difficulté toutefois, comme s'il y eût une grande irritation de la gorge et du pharynx. - Le lendemain 
matin, il avait sa conuaissance, mais était assoupi. Pouls 100. Je Ie laissai garder le lit et quelques jours après il allait très ljien.

Rem. - Il y a lieu de se demander si l'enfant en question n'étail. pas un épileptique chez lequel l'ingustion d'Armm aurail dretermini un accès.

IV. (Fnasen). - John Sides, âgé de 8 ans, fut trouvé sur le chemin dans le mème état que J. Call. Convulsions, pupilles dilatées, il ne pouvait marcher, mais pouvait voir où il était conduit. Les spasmes n'étaient pas si violents que ceux de J. Call.

Je lui donnai un vomitif de sulfate de zinc et d'ipeca, qui amena un vomissement de matières verdâtres, ressemblant à des épinards hachés; après cela, il but du café brùlant avec beaucoup d'avidité; la première tasse fut vomie, la deuxième gardée. Il se remit si vite, que 2 heures après il était capable de retourner chez lui à pied. Je preserivis qu'il se reposît et prît une purgation de calomel et jalap. Ce médicament rendit l'intestin libre et le lendemain il était guéri.

V. (Alliot). - Le 9 avril, j'étais appelé, à 6 h. 35 du matin, pour me rendre près de M. A. M., petite fille de 4 ans.

Quand j'arrivai à 7 h. $1 / 4$ je la trouvai morte: la peau entièrement marbrée, la raideur de la mort établie, le corps presque froid. Elle était morte à 5 h. 30 du matin. On me raconta qu'elle avait cessé de jouer le vendredi à 3 h. $1 / 2$ de l'après-midi, se plaignant d'être fatiguée. Sa mère la coucha, et elle s'endormit une première fois. A 5 h. $1 / 2$, elle s'éveilla, prit du thé avec un peu de lait qu'elle vomit immédiatement, ainsi que quelques matières caséeuses. Elle se recoucha, et eut un sommeil fort agité jusqqu’à $10 \mathrm{~h} .30 \mathrm{du}$ soir. A ce moment elle se réveilla avec des vomissements et une forte diarrhée. Cet état dura, accompagné de douleur, jusqu'à 5 heures du matin, elle eutalors une légère convulsion, et mourut à כ̆ h. 30 , alors que sa mère commençait à la croire profondément endormie, et dut ètre avertie par un assistant.

D'après l'ordre du coroner, je fis l'autopsie le dimanche, 3 heures après la mort. La raideur persistait encore, tous les organes étaient normaux, sauf la base des deux poumons qui était congestionnée. Les deux ventricules du cœur étaient vides et fortement contractés ; l'estomac et l'intestin grèle étaient recouverts d'une couche épaisse de mucus et de bile, pas: de sang. Le gros intestin contenait un peu de la plante (feuille) fatale qui fut encore trouvée dans les matières, et avait dù passer dans les selles précédentes.

Ceci, avec la contraction du cour, constituait la principale preuve de la nature irritante du poison qui avait donné la mort. 
Les parents avaient considéré la maladie comme une manifestation bilieuse un peu sérieuse, et ne s'étaient alarmés qu'au moment où la mort était imminente. L'enfant, avertie par une petite fille plus âgée, de ne pas manger de cette plante, en avait quand même voulu goùter.

Nous insisterons plus loin sur l'intoxication par l'Oseille et la confusion qui peut s'établir entre cette plante et les Arö̈dées indigènes.

La confusion entre ies feuilles d'Arum et celles d'Oseille peut en effet être faite par des enfants; elle semble surtout facile lor'sqüil s'agit des premières feuilles émises par les jeunes pieds d'Armm issus, soil de graines, soit des petits bourgeons tubériformes qui se délachent chaque année des gros tubercules. L’abondance, dans la plupart des régions de la forme immaculée est de nature à faciliter cetle méprise, qui ne pourrait, en aucun cas, s'établir entre les feuilles d'oseille et les feuilles largement tachetées de pourpre, de la variété maculée d'Arum. La feuille d'Arisarum, même celle des pieds àgés, n'est pas sans affecter aussi une certaine ressemblance avec la feuille de Rumex. Cette remarque ne semble pas avoir été faite jusqu'ici, ce qui donne à penser qu'aucun accident d'intoxication par cetle feuille n'a été, jusqu'ici, causé par cette confusion. La présence de petites taches violacées sur le pétiole, est d’ailleurs un caractère différentiel qui frappe à première vue. Il serait superflu d'insister ici sur les caractères qui permettent, dans tous les cas, de distinguer la feuille des Arö̈dées indigènes de celle des Rumex; indépentamment de leur forme générale et de leur dimension, sitôt leur sortie du sol (moment où la confusion doit être surtout facile pour les enfants), ces feuilles présentent 2 caractères différentiels, capables d'éviter toute méprise : $1^{\circ}$ toutes deux ont un pétiole muni, à sa base, d'une expansion membraneuse, mais en forme de gaine enroulée en cornet chez les Aroïdées (fig. 1, pl. I) d'une double languette presque étalée (Ocréa), chez les Rumex (fig. 40, 2, p. 159); $2^{\circ}$ la feuille est enroulée en cornet sur elle-même (à pointe supérieure) chez les Arum (fig. 4, pl. I), à bords seuls enroulés en dehors chez les Rumex (fig.10, 2, 3, p. 159). N'insistons pas 
ici sur les caractères qui ne peuvent être appréciés par tout le monde, et sur lesquels nous reviendrons au chapitre VIII.

\section{Intoxication par les fruits.}

Les fruits d'Arum, malgré l'odeur spéciale et plutìt légèrenent nauséeuse qu'ils répandent, lorsqu'on les écrase, ont lenté des enfants qui se sont empoisonnés en les ingérant (Connerix).

Cette opinion s'accorde mal arec l'opinion des auteurs qui prítendent que, si on applique sur la langue un fragment de fruit mùr d'Arum, on éprouve une sensation comparable à celle que provoquerait la piquire de nombreuses aiguilles, et que celte sensation peut persister plusieurs heures.

Chez les enfants intoxiqués par les fruits d'Arum, on voit se manifester une superpurgation, des crampes, des convulsions, des douleurs stomacales intenses, avec sensation de brùlure à l'arrièrebouche et à l'épigastre. La mort surviendrait de la dixième à la vingtième heure.

En réalité, les fruits mûrs d'Arum présentent une odeur plutôt désagréable, mais légère, une saveur piquante, si le péricarpe est écrasé par la mastication, mais peu accusce, si le fruit est avalé d'un seul coup. La saveur sucrée, la belle couleur rouge semblent bien de nature à tenter des enfants, qui pourraient avaler gloutonnement des fruits entiers, en quantité suffisante pour déterminer une intoxication; celte intoxication aurait toujours un pronostic grave chez les enfants; empressons-nous cependant d'ajouter qu'aucune observation précise, relative à l'intoxication par ces fruits, ne semble avoir été publiée jusqu'à ce jour.

Cherchons à dégager de ces observations les faits les plus saillants, relatifs à l'intoxication déterminée chez l'homme par les Arum. La mort (lorsqu'elle se produit) arrive au bout d'un temps variable (12 à 15 heures après l'ingestion de la plante). D'une manière générale, ce sont les symptômes gastro-intestinaux qui dominent, et ils commencent à se manifester dès le dél)ut de l’intoxication. Dans certains cas cependant, ils napparaitraient que tardivement, sans que rien puisse expliquer la cause de ce retard. Ces 
phénomènes consistent en congestion, tuméfaction, dysphagie, sensation de brûlure des premières roies digestives la luméfaction, semble en contradiction avec ce que nous a montré l'intoxication chez les animaux). L'irritation buccale el pharyngée semble d'ailleurs pouvoir faire défaut. Les vomissements et la diarrhée sont loin d'être constants. Les symptòmes généraux sont attribuables à des réflexes, dont le point de départ est la muqueuse gastro-intestinale. Ils consistent en dépression du pouls et des mouvements respiratoires, convulsions, insensibilité et coma final. Il est à remarquer que parfois l'intoxication rappelle singulierement, par certains points essentiels, l'intoxication par la cicutine, telles sont les convulsions et la mydriase observées au délut, le tremblement qui apparait plus tard (caractéristique de la période de retour dans l'inloxication par dose faible de cicutine), enfin la mort par asphyxie après convulsion finale.

Dans ses grands traits, l'intoxication spontanée chez l'homme correspond assez bien à celle déterminée expérimentalement chez les animaux; les lésions constatées à l'autopsie présentent la même analugie.

Il n'y a cependant pas à méconnaître une contradiction, sur cerlains points, entre ces deux ordres de faits. Lanciennete des observations ci-dessus relatées, ou la manière trop succincte avec laquelle elles semblent avoir été prises, suffit à expliquer ces contradictions plutôt de détail.

\section{Traitement de l'intoxication.}

Il semble assez facile, d'après les données fournies par l'expérimentation et l'analyse chimique, d'instituer, le cas échéant, une therapeutique rationnelle de l'intoxication par les Aroüdées indigènes.

La détermination de la plante, dont l'ingestion a ainsi déterminé des accidents, sera en général facile par l’interrogatoire du malade, deson entourage, el l'examen des organes entiers ou en fragments, qui peuvent se trouver dans les vêtements de l'enfant ou ćtre rejetés par le vomissement. Les quelques caractères botaniques essentiels 
peuvent seuls donner aux praticiens de's régions oir ces intoxications sont à redouter des éléments de délruminalion, assiez rapides pour combattre, en temps voulu, l'intoxication.

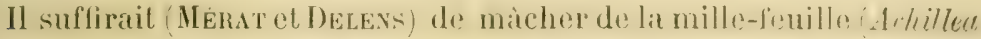
mitlofolium! pour apaiser i l'instant la cuisson violente "atusion par la mastication des fruits mûrs d'Arum.

Obtenir le rejet des débris ingérés, par le romissement, ast uno intervention tout indiquée.

" Un géomètre qui éprouvait des maux d'estomac horribles, pour avoir mangé des fruits de l'Arum, ne sentant aucune amelioration, après avoir avalé plusieurs verres d'eau fraicho, se tira heureusement d'affaire en se titillant l'arrière-bouche avec les doigts, et rendit le poison. " On comprend moins comment " un botaniste provençal, qui avait màché des fruits d'Arum, tourmenti d'une soif ardente, pris d'un flux incessant de salive gluante, après avoir demandé du soulagement à toutes les plantes qu'il reneontra sur sa route: oseille, patience, pimprenelle, chicorée, vit toute inflammation disparaître, comme par enchantement, après qu'il eut màché quelques sommités de tliym ». (Massé.)

On a recommandé d'administrer le lait, à titre d'adoucissant. C'est une médication, au moins anodine, et, s’il est toléré en assez forte quantité, ses propriétés diurétiques pourraient aider à l'élimination du toxique (alcaloïde).

On a conseillé les boissons mucilagineuses ou huileuses, les lavements d'huile : leur efficacité est bien douteuse.

On ne voit pas très bien à. quel titre employer les bains tièdes, le cataplasme de tabac et d'eau-de-rie, et surtout la saignée, recommandée par les vieux auteurs, en particulier par BuLliARD.

Des considérations exposées au chitpitre $\mathrm{V}$ sur le mécanisme de la mort dans cette intoxication découlerait une conséquence pratique.

Lorsque l'intoxication est entréc dans sa phase définitive, l'apport insuffisant des stimulations, envoyées de la moelle vers le centre bullo-respiratoire, a pour conséquence la dépression de la fonction respiratoire. La moelle, privée de son excitabilité normale, 
ne transmet qu'avec peine les excitations venues de la périphérie, il est donc indiqué d'augmenter l'intensité de ces dernières.

Les excitations portant sur les téguments, les divers modes de respiration artificielle seraient donc tout indiqués, et vraisemblablement, d'une réelle efficacité.

Le coeur, toujours affaibli, sera avantageusement renforcé par le café, ou mieux les injections de caféine.

Bibl, 1798. - Bulliand, Hist. des plantes vénén. et suspect. de la France (2 $2^{\circ}$ édit. p. 188-92).

Roques. Phylographie médicale, t, I, p. 65.

1860. - Cangella. Gaz. medicale di Porto. - Pharmaceut. Journal.

1861. - Frazer (W.) Abstract of cases of Poisoning by Arum maGulatum. (Brit. Med. Journ. n. 5, 1, p. 644-655, London.)

1881. - Alliott (А. J.). Anum maculatum. (Brit. med. Journ. n. 5, 1, p. 668. Correspond. London.)

Massé (J.). Bot. médicinale. 


\section{CHAPITRE VII}

\section{L'INTOXIGATION PAR L'OSEILLE (Rume.r).}

\section{Comparaison avec l'intoxication par les Arum.}

La possibilité d'une confusion entre les feuilles d'Arum et celles de l'oseille nous amène à dire quelques mols d'un sujet encore fort peu connu, l'intoxication possible par cette dernière plante.

L’oseille ordinaire (Rumex acelosa), cultivée, n'est généralement pas considérée comme toxique: son emploi journalier dans l'alimentation en est la preuve. Dans certaines conditions, encore mal définies, son ingestion détermine cependant de graves malaises, parfois même une intoxication mortelle. L'habitude fréquente qu'ont les enfants de mastiquer les feuilles d'oseille fait qu'un réel intérêt pratique s'attache aux intoxications, que cette plante est susceptible de déterminer.

C'est la littérature anglaise qui nous fournit la relation de cas d'intoxication par cette plante, dont certains furent mortels.

Leur relation mérite d'être rapportée avec détail.

\section{Cas mortel d'intoxication par l'Oseille (Sucking).}

J'étais appelé, dit cet auteur, à voir Edwin Arthur Geary, garcon âgé de 5 ans, le 10 juin 1886 , à 10 heures du matin. Il avait été frappé subitement.

Le malade avait été soigné par un voisin et était sans connaissance, la face froide et pâle, le front moite, les extrémités froides, la respiration lente, le pouls fréquent ef faible. La prostration était grande. Il n'y avait pas eu de convulsions. La conjonctive était légèrement sensible au toucher, les pupilles petites, mais pas très contractées, et plus tard elles derinrent très dilatées.

Le père me dit que l'enfant avait bu de l'eau savonneuse, recueillie au porte-savon, sous le couvercle perforé qui supporte le pain de savon.

Un voisin ajouta qu'il avait mangé la veille au soir une assez grande quantité de tiges fleuries d'oseille verte ordinaire (Rumex acetosa), puis pris un morceau de plum-cake. 
Alors il s'était couché, le matin suivant on l'avait vu boire l'eau savon. neuse et, peu de temps après, il s'évanouissait.

La domestique le porta en bas à son père, qui lui donna comme vomittf de l'eau salée; ce vomitif resta sans effet. A mon arrivée, je le fis placer sur un lit et produisis le vomissement avec une plume. Au bout de $3 / 4$ d'heure, il vomit $1 / 2$ litre d'un liquide transparent, sans couleur ni odeur, contenant très peu de mucus, ni sang, ni parcelle de la muqueuse. Je lui donnai une cuillère à café de vinaigre dilué, afin de neutraliser l'alcalinité du savon, puis du brandy, ordonnai l'enveloppement ouaté des jambes et un cataplasme sinapisé sur la poitrine. Peu après, il eut un copieux romissement; son pouls était alors faible et intermittent.

L'examen des matières vomies, vertes, montra une grande quantité de tiges ressemblant aux tiges d'oseille. Une petite quantité de brandy fut administrée par la bouche, et par le rectum, et de la craie fut administrée en même temps.

$\Lambda 2$ heures, je quittai le patient. Il y avait des signes de réaction; le pouls était plus plein, régulier et de 120 par minute.

Je vis le petit malade encore à 5 heures. Il était toujours inconscient et dans le coma. Selles et vomissements avaient été abondants durant mon absence.

Il mourut à 5 h. $1 / 2$, sept heures et demie après ma première visite.

L'autopsie fut faite par moi, le 14 juin, 49 heures après la mort. La raideur cadavérique était encore bien marquée. Aucune trace extérieure de décomposition. Les lèvres étaient cyanosées, mais aucune érosion, ni tache sur la muqueuse de la bouche ou de la gorge. La dure-mère était injectée, mais saine, ainsi que les autres parties du cerveau, sauf les veines de la pie-mère qui étaient tout à fait turgescentes. Le cerveau mème était congestionné, mais les autres parties absolument saines. L'estomac était vide et normal, sauf à une petite place dans la région du pylore. L'intestin grêle contenait un liquide jaunâtre et des tiges ì demi digérées. Ces tiges étaient côtelées, et avaient l'aspect de tiges d'oseille. Quelques-unes avaient 2 pouces $1 / 2$ de long. Le cœur était llasque, vide, normal.

Les poumons étaient un peu congestionnés, à la base et sur leur bord postérieur. Le foie et la rate étaient congestionnés. L'état du cœur et des viscères était celui de la mort dans le collapsus. On pensa que la mort était due à l'oseille, et avait été précipitée par l'ingestion de l'eau savonneuse. Je demandai que les vomissemeuts, l'urine, le contenu de l'intestin, l'eau, le savon, une partie des autres viscères fussent analysés. Ils le furent par le $D^{r}$ Bostock IIill, professeur de toxicologie au Collège Royal, qui me dit qu'il ne pouvait trouver aucun autre poison dans ces viscères que l'acide oxalique.

Quelle est donc la cause de la mort en ce cas? L'enfant très bien portant a mangé une grande quantité d'oseille, ver's 7 heures du soir. Il ne se plaignait d'aucun malaise ni de nausée jusqu'au matin, où, évidemment 
malade et altéré, il but l'eau savonneuse. Il but environ quatre onces d'eau, et fut trouvé quelques minutes après étendu sur le parquet sans connaissance.

Il semble donc que le poison avait séjourné toute la nuit sans être absorbé, et que le liquide avalé favorisa rapidementt son absorption, d'où collapsus immédiat.

\section{Cas d'intoxications par l'Oseille (IIANKS).}

I. - J. P., robuste petit garcon de six ans, avait, depuis quelques jours, mangé de l'oseille, mais en quantité négligeable.

En ayant absorbé une plus grande quantité, le matin du 7 juin, il ne put déjeuner à l'heure habituelle, et se plaignit de maux de cœur et de lourdeur de tête. Néanmoins, il alla à sa classe.

Vers trois heures il tomba de son siège, qui était un peu élevé : on le releva et on le porta en plein air. Les membres inférieurs étaient sans force; l'enfant vomit une certaine quantité de matière verdâtre, se leva, mais en pouvant a peine marcher. On le reconduisit chez lui, et on le coucha. Il avait toute sa connaissance, et se plaignait de la tête, et un peu plus tard d'une douleur à l'extrémité inférieure du sternum, puis il eut une toux brève, entrecoupée, et suivie d'une forte expectoration.

La personne qui le soignait, s'étant absentée quelques instants, le retrouva en proie à une crise de convulsions : les bras étaient élevés et se débattaient en tous sens, les jambes également, et l'enfant frappait sa tête avec ses mains fermées et serrées. Les globes oculaires étaient brillants, fixes, et proéminents, les dents grinçaient, mais la bouche était sans écume, et la sensibilité n'existait plus. Cet état dura un quart d'heure. Un peu avant la fin de l'attaque, la grand'mère avait donné à l'enfant une tisane de Rue qui avait paru le ranimer, et lui faire retrouver ses sens. Une sueur profuse couvrait tout le corps.

Parmi les autres symptômes, on notait un gémissement continuel, une soif ardente, de la chaleur, de la rétention d'urine, et des évacuations alvines.

Le lendemain, Je gémissement s'arrêta, mais à part cela, l'état resta le même jusqu'au 9 au soir.

Le 11; l'enfant qui n'avait pas bougé changea de position, la toux et la douleur du creux épigastrique persistèrent, le pouls était faible, la langue mauvaise. Je restai persuadé que l'estomac contenait quelque poison irritant, mais je ne pouvais avoir aucune information sur ce point.

Le 13, on me dit seulement que l'enfant avait mangé de l'oseille.

En examinant la gorge, elle était, aussi loin qu'on pouvait voir, rouge, enflammée, la portion antérieure du pharynx était recouverte d'un épais mucus.

En demandant à l'enfant de me montrer où était exactement le mal, il dirigea son petit doigt sur le trajet de l'œsophage. Pendant la déglu- 
tition la douleur augmentait; il restait couché sur le même côté, buvant avidement, le ventre n'était pas ballonné, mais l'examen en était difficile, l'enfant se contractant à la moindre pression, les extrémités et la peau étaient chaudes, la respiration devenait libre, le pouls restait petit et faible, la langue blanche et épaisse.

Dans l'après-midi du 14, après avoir mangé un œuf, il eut un vomissement dans lequel se trouvèrent des tiges verdâtres. Le vomissement fut suivi d'une crise paroxystique avec gémissement, agitation, fatigue, mais le 17, la température était normale, la douleur d'estomac moins forte. Le pharynx et la toux semblèrent les derniers à s'améliorer, mais la soif se calma, les urines précédemment très diminuées augmentèrent, l'action fébrile cessa.

Le 18 , il restait encore une certaine irritation de la gorge, et surtout une grande faiblesse. Je la combattis par du sulfate de quinine et de l'eau de roses.

Le 21, l'enfant jouait de nouveau avec ses petits camarades.

II. - Il est assez curieux de rapprocher un cas semblable arrivé à I'oncle même re cet enfant, vingt-neuf ans plus tôt. Ayant mangé et cueilli de l'oseille, il revint chez lui a vec une douleur d'entrailles soudaine, et si violente qu'il se tordait sur son lit, l'air hagard et les yeux hors de la tête. Un médecin, mort maintenant, ordonna un vomitif énergique qui provoqua l'expulsion d'une grande quantité d'oseille. Les vomissements recommencèrent trois fois, contenant de moins en moins de matière verte; la solf était intense. Le lendemain le malade était relativement bien, sauf une grande fatigue.

L'oseille ne contient comme principes toxiques actuellement connus que des sels de l'acide oxalique.

L'oxalate de chaux insoluble qui, existe en abondance dans la plante, sous forme de sphéro-cristaux, ne peut donner lieu à une intoxication. Il ne peut être absorbé que peu à peu, à mesure que sa décomposition partielle s'effectuerait sous l'influence d'ICCl du suc gastrique, l'élimination de l'acide oxalique ainsi absorbé semble se produire parallèlement à son absorption, et sous forme d'oxalate de soude, susceptible d'être décelé dans les urines.?

On trouve, dans le suc de Rumex acetosa, deux oxalates de polasse :

Le bioxalate $\mathrm{C}^{2} \mathrm{HKO}^{4}+2 \mathrm{H}^{2} \mathrm{O}$ et le quadroxalate.

Le mélange de ces deux sels forme le sel d'oseille, dont la majeure partie est constituée par le bioxalate. 
Le bioxalate n'est soluble que dans 40 parties d'eau froide, le quadroxalate l'est encore moins.

La proportion de bioxalate n'est que de $7,3 \%$ dans la plante fraîche d'après Mitscaerliscr, de $2 \%$ d'après Orfila.

L'ingestion de 11-15 grammes de bioxalate de potasse peut amener la mort. On a cependant vu l'ingestion de 30 grammes de ce sel ne pas être suivie de mort (mais dans ce cas; il y avail eu des vomissements répétés).

Ces chiffres se rapportent à des adultes; la dose mortelle est évidemment plus faible, s'il s'agit d'enfants.

Si on admet le chiffre indiqué ci-dessus pour la teneur en oxalates de l'oseille fraîche, on voit qu'il faudrait ingérer $600 \mathrm{gr}$, au minimum d'oseille crue, pour voir apparaître des accidents, en admettant même que la totalité du sel ingéré passe dans la circulation générale. Tel ne doil pas être le cas : car, à cause même de la faible solubilité de l'oxalate de potasse, une notable proportion du sel ingéré ne doit pas être absorbée, et passe peutêtre, en présence des sels de chaux absorbés avec les aliments, à l'état d'oxalate de chaux, totalement insoluble. Il ne semble pas que la faible quantité d'oxalate de potasse susceptible d'être absorbée, après ingestion d'oseille, soit capable de déterminer des accidents, dans les circonstances ordinaires; l'expérience journalière en est, du reste, la preuve.

Dans le cas d'intoxication ci-dessus relaté, on peut être en droit de supposer que l'ingestion consécutive d'eau saronneuse a permis ì l'oxalate de potasse, peu soluble, de passer à l'état d'oxalate de soude, plus soluble, par suite plus facilement absorbable. Nème dans cette hypothèse, il semble difficile d'admettre que la quantité de plante ingérée ait été suffisante pour introduire dans le tube digestif une dose mortelle d'oxalate.

Les symptômes observés au cours de l'intoxication par l'oseille et les lésions constatées a l'autopsie concordent-ils avec ceux de l'intoxication par les oxalates?

Dans l'intoxication par l'oseille, les symptômes dominants sont: 
nausées, céphalalgie, refroidissement et pâleur des téguments, rougeur el douleur dans l'oesophage, les fosses nasales, difficulté de déglutition, respiration lente, pouls fréquent et failble, puis intermittent, diminution de la sensibilité, mydriase, conrulsions dans certains cas.

Et les lésions : congestion des poumons, du foie, surtout de l'encéphale (la coagulation ou la fluidité du sang n'a pas été notée).

L'oseille agirait à la façon d'un irritant direct du tube digestif : la toux, signalée chez les intoxiqués par cette plante, serait une toux d'origine gastrique, et les convulsions seraient attribuables ì un réflexe, dont le point de départ serait les terminaisons motrices intestinales du sympathique irrité.

Il existe une analngie réelle arec les symptômes et les lésions de l'intoxication par les oxalates (1).

L'ingestion d'oxalate de potasse détermine : une douleur stomacale brûlante, de l'anxiété précordiale, des défaillances, des vomissements nombreux et violents dans certains cas, pouvant manquer dans d'autres, un abattement considérable, le refroidissement, lit dépression du pouls, du délire, parfois des convulsions, la mort arrive par syncope en quelques heures. A l'autopsie, le sang est vermeil, les poumons congestionnés, les divers viscères présentent des extravasations sanguines, l'estomac est parfois indemne de toute lésion.

Linjection intraveineuse d'oxalate de soude provoque une respiration haletante, une accélération et une diminution d'amplitude des battements cardiaques, le cœur cesse bientôt d'être susceptible, et après de profonds soupirs la respiration s'arrête : la mort arrive donc par syncope. A l'autopsie, le cœur a ses cavités gauches remplies de sang rouge, ses cavités droites de sang noir; le sang est partout fluide, incomplètement coagulable, mème à la longue (caractères du sang oxalaté, théorie d'Artus sur la coagulation).

La même remarque s'applique aux intoxications observées dans (1) V. Ranuteau (A.), Élém. de toxicologie, 2e éd. Paris, 1887, pp. 792-93. 
certains cas chez les animaux domestiques, a la suite de l'ingestion de la petite oseille sauvage (Rumex acetosella).

La petite oseille est mangée avec plaisir par les moutons, d'où son nom vulgaire: Oseille à brebis. Mais on l'a vue produire des intoxications chez le cheval et le mouton, lorsqu'elle est broutée couverte de ses graines.

Le tableau symptomatologique de cette intoxication, chez le cheval, est le suivant (MicmeLs):

$1^{\text {re }}$ période. - Démarche titubante, écartement des membres pour maintenir l'équilibre, anxiété, lèvres pendantes, plyalisme, cyanose des muqueuses;

$2^{\text {e }}$ période. - Frémissements musculaires des fessiers, des scapulo-olécraniens, mydriase, relichement des sphincters, émission d'urine, pouls lent, faible, avec des intermittences de cing en cind minutes.

$3^{\mathrm{e}}$ période. - Contraction convulsive des lèvres, rétraction de l'œil an fond de l'orbite, respiration accélérée, stertoreuse, dilatation extrême des naseaux, contracture létanique des muscles de l'encolure, du dos, des membres, soubresauts convulsifs, sueur abondante, chute. L'animal se relève, reste quelque temps abattu, puis une nouvelle attaque survient, et l'animal expire dans une dernière convulsion.

A l'autopsie, on ne constate, à peu près, qu'une inflammation marquée du sac droit de l'estomac.

On en est réduit à de simples hypothèses sur la nature du principe vénéneux que renfermerait la petite oseille. Serait-ce l'oxalate de potasse? Le tableau symptomatique plaiderait assez en faveur de cette hypothèse.

Il està noter cependant, que les accidents provoqués par la petite oseille l'ont été par la plante fleurie ou fructifiée. Or le sarrasin (Polygonum Fayopyrum L.) détermine, surtout à l'époque de sa floraison, desaccidents bien connus, sur les bestiaux qui le broutent, ou peutêtre mème sonl soumis aux émanations de ses sommités fleuries. La paille et les graines provoquent aussi, sur les animaux qui les consomment, une série de phénomènes congestifs. 
Les graines de Polygonum convulvulus peuvent aussi déterminer, chez le cheval, des désordres qui se traduisent par une entérite plus ou moins grave.

Dans l'intoxication provoquée par l'oseille chez l'homme, c'est aussi la plante fleurie qui est incriminée.

La toxicité, ou tout au moins la toxicité maxima des Polygonées indigènes, semble donc coüncider avec l'époque de floraison de la plante. Ce fait s'accorderait difficilement avec l'hypothèse qui attribue à l'oxalate de potasse la cause de l'intoxication; car ce sel existe en abondance, dès les premiers temps de la végétation, dans les feuilles et les jeunes tiges.

Il est une autre remarque importante, on ne signale nulle part des symptômes fâcheux, à la suite de l'ingestion de l'oseille cuite, les quelques cas d'intoxication connus chez l'homme se rapportent à des enfants ayant mâché de l'oseille crue. La destruction, par la cuisson, du principe toxique ne concorde guère avec l'hypothèse qui altribue aux oxalates la cause de cette.intoxication.

(On a d'ailleurs cité (CLaRle), ce fait qu'en certaines contrées de la Suède, en temps de disette, les paysans mangeaient avec le pain des quantités assez considérables d'oseille crue, sans en éprouver d'inconvénients.)

Peut-être est-il plus prudent de conclure que le principe toxique des Polygonées indigènes, et en particulier de l'oseille, reste à découvrir.

Bibl. 1847. - Hanks (H.) The injurious effects of Rumex AcEToSA. (Lond. med. Gaz., n. s. V, pp. 69-82.)

TAYLoR Medical Jurisprudence 2 ed., p, 103 (relat. des cas de Hanks).

1863-66. - MoISANT. Effets de la paille de sarrasin sur le mouton. (La culture, p. 259.)

1870. - GaIATA (DE). Effets du sarrasin en fleurs sur le bétail. (Joürn. d'agricult. pratique, t. I, p. 531.)

1870. - Michels. Empoisonnement par le Rumex, petite oseille. (Ann. de méd, vétérinaire de Bruxelles.)

1872. - Brodnax (D. W.), Death from poisoning by « sorrel » (Rumex acetosa) (Richmond and Louisville med. J. Louisville, XIV, p. 82.) 
1875. - Fromet. Mème sujet (ibid., t. II, p. 363 et p. 517).

1886. - Bienman (B.) Zuring vergiftiging bij het schaap. Tijidschr. $v$, veeartsenijk, en vee teelt. (Amst. XIII, p. 197).

Chassaing. Empoisonnement par la petite oseille. (Bulletin de la Société d'agricult. de France, 21 janvier.)

Suckling (C. W.). Cas d'intoxication par Rumex acetosa. (Detroit Lancet (édité à Detroit), II, 5, p. 227).

ID. (Brit. med. Journal, p. 436).

1087. - Cornevin (CH.). Des plantes vénéneuses el des empoisonnements qu'elles déterminent. (Bibl. de l'enseignement agricole, in- $8^{\circ}$. Firmin-Didot, Paris, p. $151-156$.

1887. - Galtier. Entérite déterminée par des graines de Polygonum convolvulus. (Journ. de méd. vétérinaire de Lyon.)

Clarke (in the London dispensatory, 6 th. edit, p. 564 ).

Il importe donc au médecin de pouvoir reconnaître et déterminer, rapidement, et avec exactitude, des fragments plus ou moins considérables des organes aériens de Rumex acetosa. Cette détermination devra être faite, soit au lit du malade, s’il s'agit d'instituer le traitement de l'intoxication, soit dans une expertise médico-légale.

Pour cette dernière, les caractères histologiques seront de sùrs documents de détermination, alors même que la plante serait réduite en fragments minuscules. Les caractères urganographiques (surtout si la plante est fructifiée: guideront seuls le praticien au lit du malade.

Rappelons donc ces derniers caractères, puis nous ferons une étude histologique de la feuille d'oseille, qui n’a pas été faite jusqu'à ce jour.

a. Caractères organographiques de Rumex acetosa.

Fleurs hermaphrodites ou polygames. Réceptacle en forme de cupule peu profonde, portant sur ses bords le périanthe trimère et l'androcée, et, à sa partie la plus déclive, l'ovaire. Périanthe a folioles extérieures égales, d'abord imbriquées, à folioles intérieures alternes, les extérieures plus grandes. Androcée de six étamines, superposées par paires aux folioles extérieures du périanthe, formées d'un filet grêle, supportant une anthère 

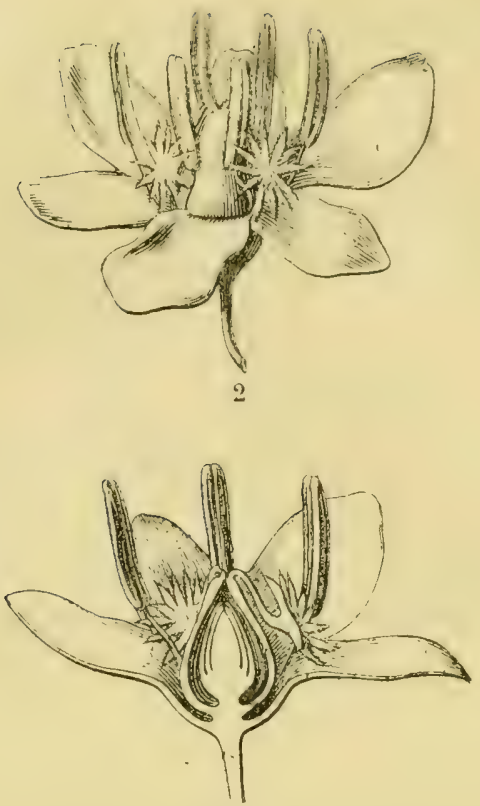

3

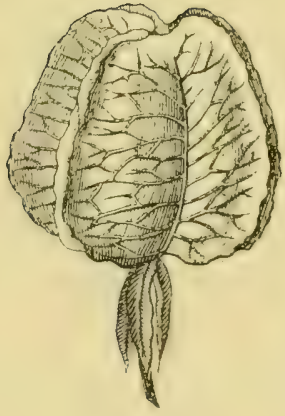

4

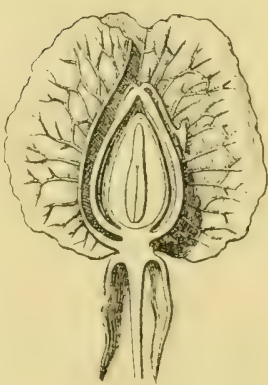

5

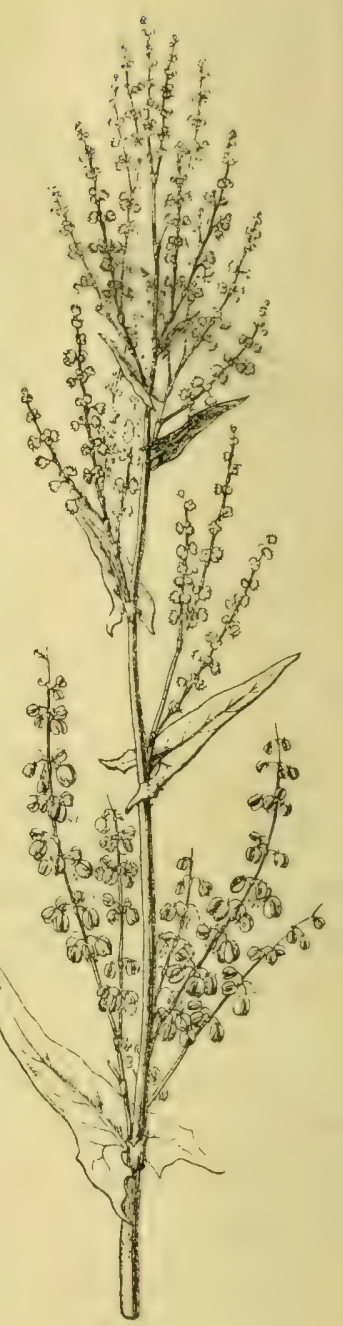

1

Fig. 39. Rumex acetosa. - 1 rameau fleuri et fructifère, 2 fleur isolée, 3 fleur isolée, c. longit., 4 fruit isolé, 5 fruit isolé, c. longit.

allongée, biloculaire, déhiscente par deux fentes longitudinales, introrse ou latérale. Ovaire libre, surmonté de trois branches stylaires, rabattues à sa surface externe, terminées par une sorte de pinceau stigmatique, multidenté, papilleux, affleurant au périanthe en dehors de l'androcée. Dans la loge unique de l'ovaire un placenta central, basilaire, portant un ovule dressé, orthotrope, bitégumenté, à micropyle supérieur. 
Fruit, dit en caryopse, à angles proéminents, surmonté des débris des trois branches stylaires, à pédoncule porteur des pièces persistantes du périanthe, les extérieures non accrues et réfléchies vers le bas, les intérieures accrues, dressées contre les faces du fruit, à jords irrégulièrement denticulés, veinées, à nervure médiane saillante.

Graine à téguments minces, à albumen farineux, à embryon petit, presque rectiligne, à cotylédons plan-convexes, oblongs, à radicule supère.

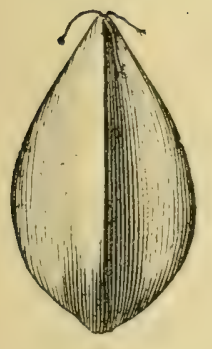

1

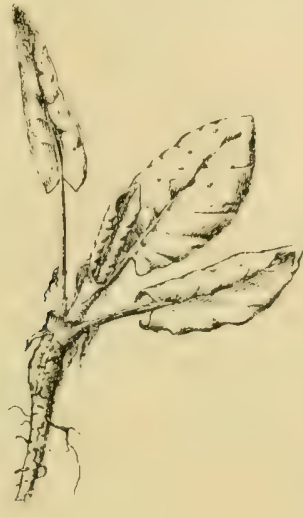

2

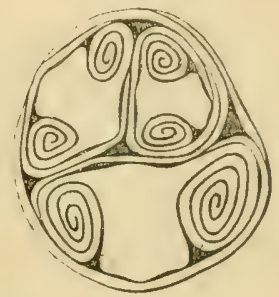

3

Fig. 40. Rumex acetosa. 1 fruit, le périanthe détaché, 2 jeune pousso d'oseille, 3 c. transvers. d'un bourgeon, pour montrer la préfoliation.

Feuilles alternes, rapprochées à la base en fausses rosettes basilaires ; limbe hasté à la base, oblong, atténué au sommet en pointe mousse, entier, à nervation pennée, à nervure médiane saillante à la face inférieure, à peu près plane à la face supérieure, à bords enroulés plusieurs fois sur eux-mèmes en dehors, dans la préfoliation (fig.40,3) (disposition encore très nette sur là feuille jeune, peu de temps après son épanouissement) (fig. 40, 3). Pétiole accompagné à la base de stipules modifiées, unies en une gaine membraneuse, entourant d'abord l'axe (ocrea).

Inflorescences, en grappes de cymes contractées, souvent unipares, à l'aisselle des feuilles réduites, puis des bractées, qui occupent le sommet des rameaux florifères.

b. Structure anatomique et histologique de la feuille de Rumex acetosa.

(Les caractères ci-dessous indiqués se rapportent à une feuille jeune, lors de son épanouissement au premier printemps. Comme ce n'est guère qu'à cette époque que la feuille d'Arum pourra ètre confondue avec celle d'Oseille, il y a tout intérêt à préciser les caractères d'une feuille à peine épanouie).

Pétiole, c. initiale (à la hauteur de l'ocrea), en fer à cheval très ouvert, à angles mousses; un faisceau médian occupe le plan de symétrie; de 

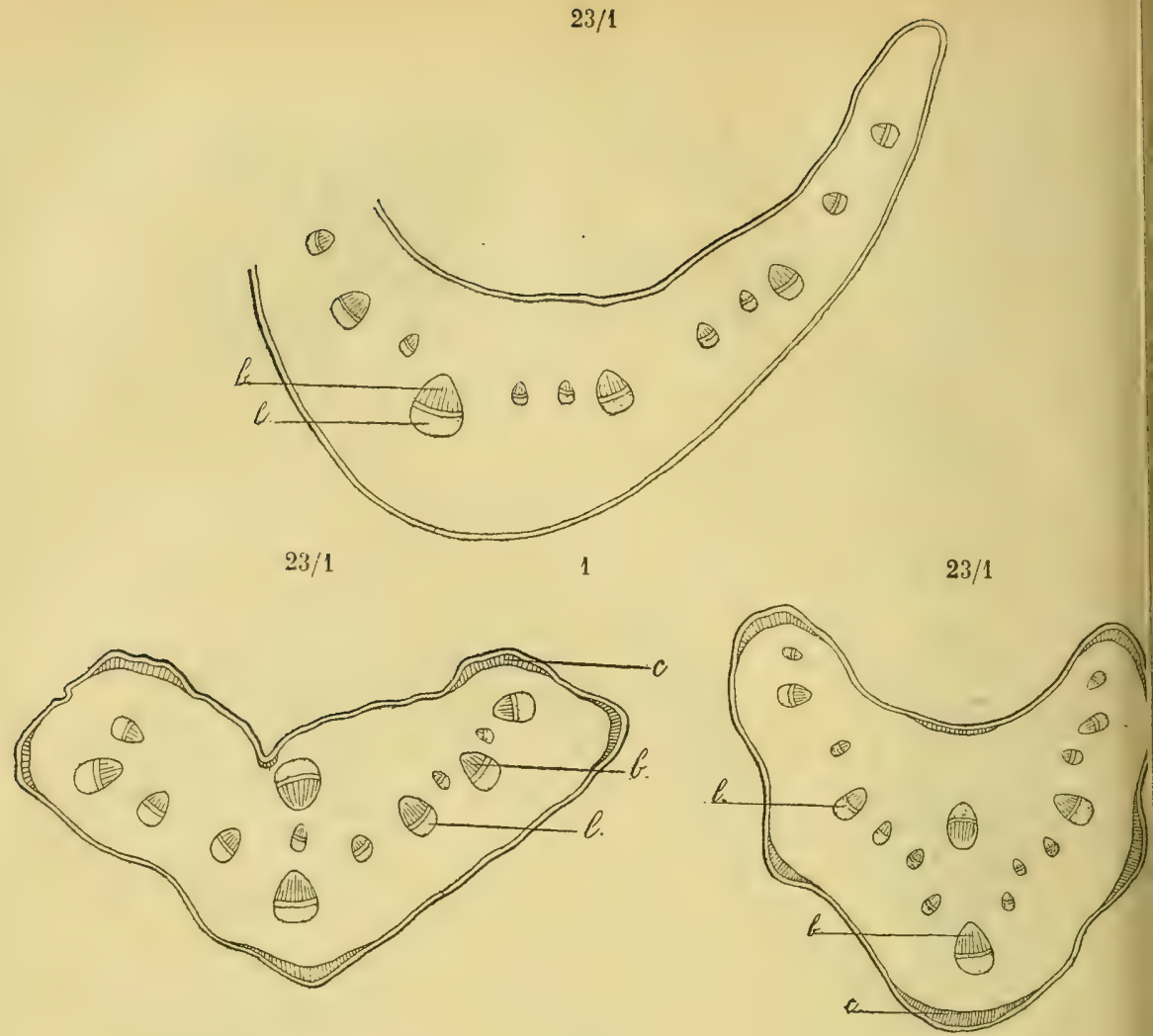

3

$4 \dddot{3} / 1$

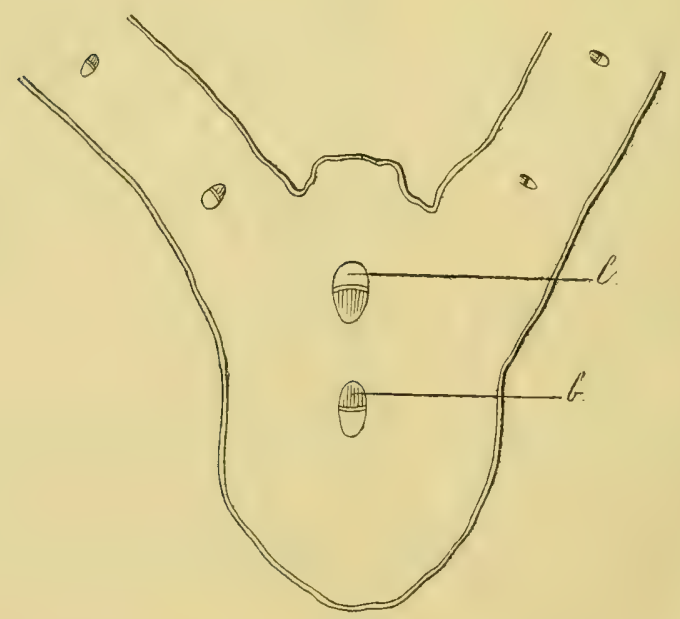

4

Fig. 41. Rumex acetosa (semi-schéma). - C. transv. du pétiole ot de la nervure méd. du limbe; 1 initiale, 2 médiane, 3 terminale.

C. transvers. du pétiole : 1 initiale, 2 médiane, 3 terminale gross. $23 / 1$ et 4 de la nerv. méd. de limbe gross. $45 / 1$ 
part et d'autre de ce plan, deux gros faisceaux; avec $\ddot{b}-6$ petits fais. ceaux alternes.

C. médiane, convexo-concave, à concavité interne, à angles supérieurs très obtus, avec deux saillies latérales de la face externe; même disposition des faisceaux qu'a l'initiale, mais un gros faisceau, à orientation inverse, occupe le plan de symétrie.

C. terminale (ou caractéristique, à la naissance du limbe) convexoconcave, à face supérieure creusée en gouttière aiguë, deux renflements latéro-supérieurs sont l'ébauche du limbe; même disposition des faisceaux qu à la médiane, sauf la réduction du nombre des petits faisceaux accessoires.

Nervure médiane (au milieu), obrectangulaire dans l'ensemble, à face supérieure et inféricure convexe; deux faisceaux sculement dans le plan de symétrie, l'un à orientation normale, l'autre à orientation inverse.

A tous les niveaux du pétiole, on observe:

Épiderme à parois minces, l'externe plus épaisse, striée, avec quelques glandes semblables à celles du limbe.

$\Lambda$ l'initiale, il n'existe pas de tissu collenchymateux, mais il en existe aux niveaux supérieurs, localisé à tous les points saillants de la surface; en tous ces points, deux ou trois assises de cellules, formant des plages collenchymateuses, à parois relativement peu épaisses, sauf aux angles, sont situées immédiatement au-dessous de l'épiderme; ces plages aflectent la forme de croissants, avec passage insensible à la couche hypodermique, que rien ne différencie, dans les points non saillants de la surface, du reste du parenchyme cortical ; une mince plage, peu étendue, de collenchyme se trouve également dans le milieu de la concavité de la face supérieure. Le collenchyme disparaît à nouveau dans la nervure médiane du limbe.

Le parenchyme cortical est uniformément constitué de cellules, assez régulières, à parois minces, séparées par les méats, larges surtout dans la zone périphérique; çà et là, les éléments de ce parenchyme renferment des cristaux en oursins (mâcles polysynthétiques) d'oxalate de chaux.

Les faisceaux, sont isolés dans le parenchyme, entourés d'une simple gaine de parenchyme peu condensé, privés de tout élément de soutien, à faisceaux réduits à des trachées, de médiocre calibre, à section circulaire.

Limbe. - Épidermes inférieur et supérieur très semblables, à éléments polygonaux, plus ou moins curvilignes (vus de face), a section obrectangulaire aplatie, quelque peu onduleuse, à parois minces, finement grenues; les cellules superposées aux nervures sont allongées dans le sens de celles-ci. Les stomates, à peu près aussi abondants sur les deux faces, sont elliptiques ou sub-circulaires, généralement accompagnés de trois cellules de bordure, situés sur le plan de l'épiderme. Quelques cellules épidermiques se différencient en glandes capitées, dont la tête, formée de quatre cellules saillantes, est supportée par un court pédicule générale- 


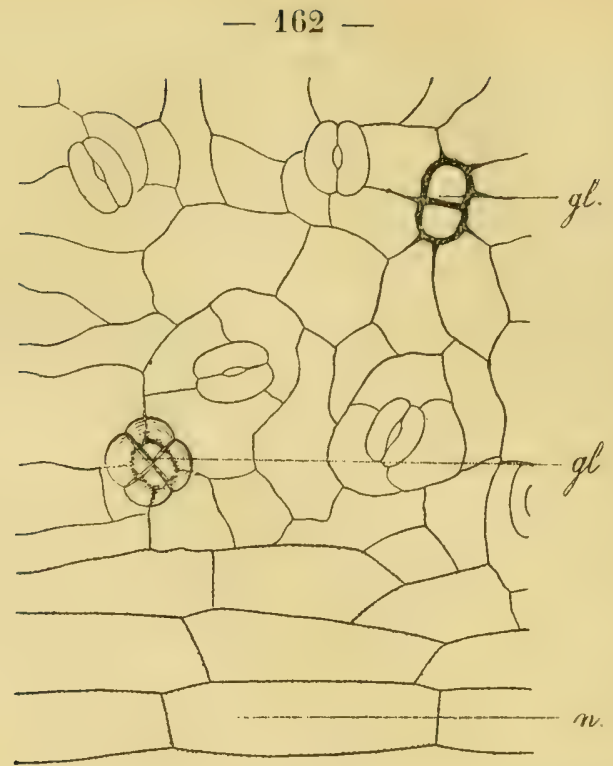

Fig. 42. Rumex acelosa (Gross. 190/1). - Epiderme inférieur du limbe; $g l$ glandes l'une, entière, l'autre détachée de ses cellules basilaire d'insertion, $n$ portion superposée à une nervure.

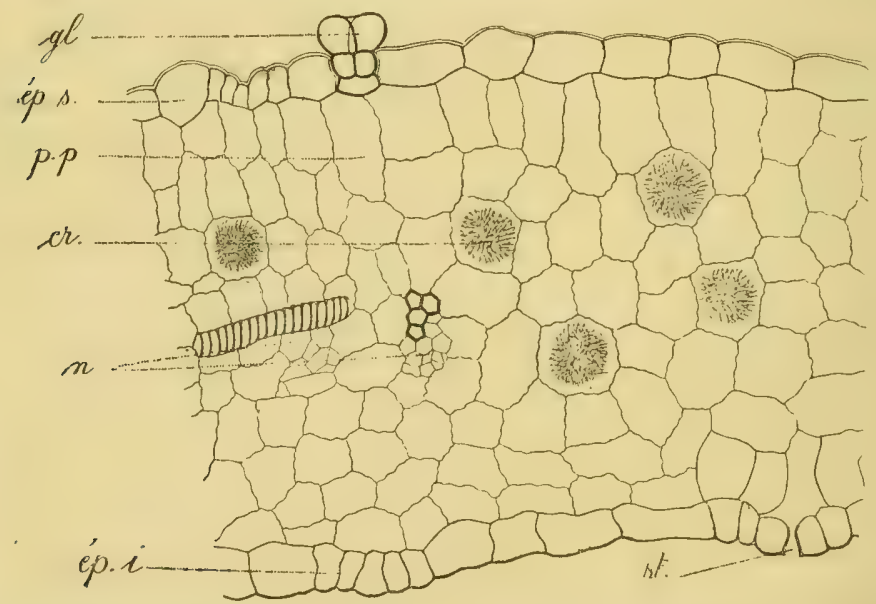

Fig. 43. Rumex acelosa (Gross. 190/1). - Limbe d'une jeune feuille (c. transv.) $g l$ glandes, $p p$ parench. palissad., $c r$ cristaux en macles, $n$ nervures, $s t$ stomates.

ment bicellulaire; les cellules basilaires, à parois épaissies, de ces glandules sont très visibles à la surface de l'épiderme, lorsque le pédi. cule qui s'y insère en est détaché. Mésophylle formé de deux couches, 
(la supérieure surtout nette,) de parenchyme palissadique, et d'un parenchyme homogène, peu lacuneux, avec nombreuses macles d'oxalate de chaux. Il n'existe aucun élément de soutien, et les nervures, disposées sur un seul plan, à égale distance des deux faces, ont leurs éléments vasculaires tous spiralés.

(Nous ne parlerons pas ici de la structure de l'ocrea, et des glandules massives qu'elle porte à sa surface, la base des feuilles ne risquant presque jamais d'ètre ingérée.)

La comparaison des caractères fournis par l'examen de débris d'Oseille pernettra toujours leur distinction, d'avec des débris appartenant à des Aroïdées indigènes.

La confusion possible des feuilles d'Oseille avec celles d'Arum devra toujours être présente à l'esprit, dans le cas d'intoxication supposée par l'une ou l'autre de ces plantes. Les symptômes de l'empoisonnement par ces plantes sont à la fois des phénomenes de gastro-entérite, dus à leur action locale sur le tube digestif et des phénomènes généraux, surtout du côté du système nerveux. 


\section{CHAPITRE VIII}

\section{LES ARUM AU POINT DE VUF MIÉDICO-LÉGAL}

\section{A. - Des prétendues propriétés abortives des Arum.}

Dans certaines régions, en particulier dans le Soissonnais, les Arum sont réputées plantes abortives, d'un usage infaillible, et employées comme telles, par les matrones, de la façon suivante (Dr Dinant com. or. Ce confrère, a recueilli, dans deux cas, la déclaration de personnes qui se sont accusées d'avoir fait usage de l'Arum comme abortif, et d'avoir perdu, à la suite de son emploi, des embryons de deux mois environ). Le tubercule lavé est bouilli, pendant environ une demi-heure, dans environ 200 grammes d'eau, l'extrait aqueux concentré et administré séance tenante pour obtenir l'effet voulu. Il est à remarquer que, d'après ce mode de préparation, la décoction d'Amm est totalement privée de raphides, restés tous dans la masse du tubercule, et d'alcaloüde éraporé par la chaleur ou entraîné par la vapeur d'eau; il ne peut contenir qu'une certaine quantité de saponine, puisque celle-ci n'est pas extrêmement soluble dans l'eau, et que le tubercule n'est pas même débité en fragments, de façon à faciliter sa dissolution. L'emploi de l'Arum, comme abortif, ne semble pas, à priori, pouvoir être d'une efficacité quelconque, ni déterminer aucun accident général.

Les expériences suivantes confirment cette opinion, formulée à priori.

Expér. LII. - Un tubercule d'Arum italicum frais, au moment de la reprise de la végétation, du poids de äs̆ grammes, est bouilli pendant une demi heure avec 200 grammes d'eau, broyé, et laissé à macérer jusqu'au lendemain. La pâte ainsi obtenue est exprimée au nouet, et l'on obtient $100^{3} d^{\prime}$ 'un suc, à consistance d'empois, qui est injecté par la sonde csophagienne à un cobaye femelle, neuve et pleine, du poids de 860 grammes; 
4 jours après, mise bas de 3 petits normaux, bien portants, dont le développement ultérieur ne laisse rien à dèsirer.

Donc, à doses relativement énormes pour la taille de l'animal, l'extrait aqueux préparé à $100^{\circ}$ avee lo lubercule d'Arum, et contenant la presque totalité des substances solubles dans l'ean à cette température, et en particulier une quantité assez notable de saponine (mousse abondamment par addition d'eau et batlige), est tolalement dépourvu d'effet, chez le cobaye, sur l'utérus gravide et sur l'état général.

Expér. LIII. - Deux tubercules frais d'Arum maculatum, un peu avant la reprise de la végétation, pesant en tout 62 grammes, donnent $100^{\circ} 3$ d'un extrait, préparé comme ci-dessus. Cet extrait est administré en totalité à une chienne, en état de gestation avancé; aucun effet. Le lendemain, la même chienne ingère avec ses aliments 6 tubercules bouillis, pesant en tout 111 grammes. Résultat nul : 3 jours après, mise bas de quatre petits vigoureux, dont le développement ultérieur est de tous points normal.

Ces expériences, répétées à diverses reprises, dans des conditions identiques, nous ont toujours donné un résultat négatif. Les propriétés abortives des Arum nous semblent devoir être totalement révoquées en doute. Mais la croyance populaire, dans certaines régions, à l'existence de ces propriétés, mérite d'être connue du médecin-légiste.

Celui-ci peut avoir à se prononcer sur l'emploi des Amu, comme abortifs, sinon efficaces, au moins utilisés dans un but crịminel.

\section{B. - Détermination médico-légale des Arum et de leurs principes toxiques.}

La détermination des Amm, au cours d'une expertise médicolégale, peut se faire, soit par la recherche et la caractérisation de leurs principes actifs: alcaloïde et saponine, soit par l'analyse histologique des débris de la plante incriminée.

\section{I. - Lésions constatées a L'autopsie.}

Nous avons trop insisté aux chapitres III et IV sur les symptimes de l'intoxication expérimentale ou spontanée par les Arum, et sur les lésions constatées à l'autopsie, pour y revenir ici. 
Si les lésions, constatées dans une autopsie, sont de nature à faire soupronner une intoxication par les Arum, il importera de recueillir arec soin tous les débris végétaux, susceptibles d'ìtre examinés ultérieurement, au point de vue d'une détermination botanique.

\section{II. - Recherche des PRINGIPES ACtifs.}

La connaissance des principes chimiques actifs de ces plantes nous dispense d'insister sur les méthodes d'extraction et de détermination à employer, au cours de l'expertise. Ce sont les méthodes classiques de recherche de la conicine et de la saponine, exposées dans les ouvrages spéciaux de toxicologie. Nais, à ce point de vue spécial, quelques réflexions trouvent ici leur place.

L'alcaloïde existe en trop petite quantité dans les Arum pour qu'à l'autopsie, on puisse percevoir l'odeur de souris qui le caractérise. Nous l'avons constaté à l'autopsie des animaux intoxiqués par le suc d'Arum (d'ailleurs ce suc lui-même exliale une odeur nauséeuse, sui generis, mais qui ne rappelle guère celle de l'alcaloïde). Ce premier criterium invoqué par quelques auteurs (nié d'ailleurs par d'autres) dans l'intoxication par la ciguë, fait ici totalement défaut.

Comme pour la conicine, il y aurait lieu de rechercher l'ulcaloïde dans le sang, les organes richement vascularisés, et dans l'urine. On se rappellera que la conicine se retrouve dans l'estomac, souvent très longtemps après son ingestion, que les intestins n'en contiennent généralement que des traces, et qu’elle semble s'éliminer en partie par les poumons (ZALEWsiy et AdELHeim) (1).

Le procédé de choix pour la recherche et l'extraction de l'alcaloïde des Arum semble ètre celui proposé par DRaGExdonfF pour l'extraction des alcaloïdes volatils. (Nous renvoyons pour les détails au traité de toxicologie de cet auteur). L'extrait par l'éther de pétrole répandra l'odeur de souris. $\mathrm{HCl}$ étendu, ajouté à cette solution éthérée, donnera grénéralement un précipité (se déposant progressivement) cristallin, en longues aiguilles ou d'apparence den-

(1) Beit. z. gerich. Chemie, p. 4'. - Unters. ü. J. Conicin, Dorpat, 1869. 
drilique. Ce chlorhydrale dera ctre examini, aussitôt après la dessiccation de la solution aqueuse qui se forme après l'évaporation de l'éther de pétrole.

Lorsque, dans une expertise médico-légale, on se trouvera en présence d'une base végélale linuide, volatile, on devar désormais songer rue l'on peut avoir aflaire à l'alcaloïde des Amm.

Il est inutile de revenir ici sur sa grande analogie, sinon identité avec la conicine. Cette analogie même ne manquerait pas, dans certains cas, que d'embarrasser l'expert appelé à se prononcer sur la plante cause de l'intoxication.

La présence dans le Piment (Capsicum) d'un alcaloüde volatil, à odeur de conicine, présentant avec cette dernière, et par suite avec l'alcaloüde d'Arum, un certain nombre de caractères communs, doit être présente à l'esprit. Celte donnée est importante, surtout dans le cas d'intoxication présuméc par les fruils d'Arum. Les fruits de Piment possèdent, en effet, un pigment rouge orangé, assez roisin de celui des Arum, et les débris du péricarpe, bien que plus coriaces (épicarpe), pourraient, à un examen superficiel, prêter à confusion. La recherche des graines, si diflérentes, comme taille et comme structure, lèvera tous les doutes en quelques instants, et, d'une façon infiniment plus précisé, que ne pourrait le faire une analyse chimique laborieuse.

Il est douteux que, dans un cas d'intoxication, même mortelle, parl'Arum, on puisse retirer, des viscères, ou du contenu du tube digestif, une quantité d'alcaloïde suffisante pour rechercher ses propriétés physiologiques.

La recherche toxicologique de la saponine semble devoir présenter aussi les plus sérieuses difficultés.

DragendorfF a posé la question de saroir si, dans la recherche toxicologique et la séparation des loxiques résicants, on ne serait pas exposé à confondre un de ces principes avec " les principes 
âcres et rolatils des rhizomes d'Arum ». Il n'a pu y répondre.

Nous sommes en mesure de le faire, et la question est d'une réelle importance. La confusion est impossible entre le principe âcre, rolatil (non seulement des Armm, mais des Arö̈dées indigìnes, et probablement de toutes les Aroïdées ûcres) et les toxiques résicants que le toxicologue peut rencontrer dans ses expertises, s'il emploie, dans ses recherches, le procérlé indiqué par Draciexdorfr, pour isoler et caractériser les substances vésicantes (1. c., p. 444).

Nous n'avons pas à rappeler ici les détails de ce procédé; souvenons-nous seulement que les matières à examiner sont soumises à l'ébullition prelongée en présence d'une lessive potassique. Or, nous arons assez repété, en relatant nos essais chimiques, qu'en présence de $\mathrm{KOH}$, le principe âcre des Arum est totalement volatil. Comme la recherche toxicologique des principes vésicants se poursuit sur la masse de matières non volatiles, en présence de $\mathrm{KOH}$, on ne peut être exposé à rencontrer, dans la suite des manipulations, la conicine des Aroïdées.

Remarquons d'ailleurs que le pouvoir vésicant proprement dit de l'alcaloïde ou du suc des Arum est absolument nul (v. p. 8ə̈-87).

Bibl. 1886. - Dragendonff. Manuel de Toxicologie $\left(2^{e}\right.$ éd. franç. par L. Gautier), p. 448 , in $-8^{\circ}$, Paris, Savy.

\section{III. - Détermination mistologieue.}

La seule manière pour l'expert d'arriver à une détermination précise de la plante incriminée est de recourir à la détermination histologique des débris, que ne manquerait pas de contenir le tube digestif.

Il semblerait même logique de commencer toujours les recherches médico-légales par la détermination des débris régétaux, trouvés à l'autopsie, dans les matières fécales ou vomies, ou dans les aliments non encore ingérés, et ayant subi des préparations culinaires.

Nous ne pouvons insister ici sur l'intérèt général de ces recherches; mais, pour nous borner à ce qui regarde les intoxications par les Arum, il est certain que la détermination histologique des débris 
de ces plantes, suserptibles d'ètre recueillis par le méderin légiste, faite par un spécialiste, sera, presque toujours, sinon facile, au moins précise.

a) Procédés techniques. - Sans parler ici des divers procédés tech-

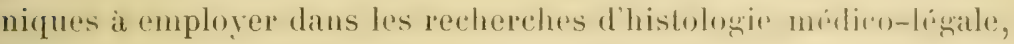
disons quelques mots de ceux d'entre eux qui sont particulièrement applicables à la recherede et à la détermination destrbris des Aroülées indigènes, susceptihles d'être rencontrées an conursthexpertises toxicologiques. Point n'est besoin d'insister sur les procédés courants pour enrober et pratiquer des coupes minces, dans les organes entiers; en particulier dans les graines, que l'ex pert pourrait rencontrer dans le tube digestif ou les matières romies. La recherche et la détermination des fragments de parties vertes, de rhizome ou de péricarpe mérite plus d'attention. Il est rare que les feuilles, pétioles, ou fruits (eussent-ils mème subi un commencement de putréfaction) soient dans un état tel, qu'ils ne puissent prêter à une détermination précise; les fragments un peu rolumineux de ces orgaues seront avantageusement placés, après lavage, diuns une solution de formol (i $40 / 0$ ); s'ils présentent encore quelque peu de consistance, ils pourront ètre débités en coupes, présentant dés sections d'ensemble de l'organe à déterminer. Dans ce cas, la comparaison avec les figures relatives aux sections transrersales des divers orsanes de li plante, permellra une détermination rapide el précise. Tel siratil le cas pour des fragments de pétiole aralés sartis ètre mìchesi, de limbe foliaire (dont des pareelles plus on moins étendues persistent toujours, mème apries mastication, de péricarpe avalé en gros fragments) el surtout de graines (qui traversent intactestoute la longueur du tube digestif. L'emploi du formol comme liquide conservateur et durcissant, présente sur celui de l'alcool le grand avantage de conserver, au moins pendant la durée de l'expertise, leur coloration naturelle aux débris recueillis.

Les parcelles de tissus franchement désagrégés par action mécanique ou digestive pourront ètre examinées par transparence; le plus souvent, il y aura avantage à les soumettre au préalable à l'action des agents éclaircissants ichloral, alcalis causliques! ou 
sourent, micux encore, à l'action des hypochloriles alcalins. Ceuxci, en détruisant toutes les matières protéiques et colorantes, permettent l'emploi des réactifs colorants í en particulier le vert d'iode acétique pour la coloration des éléments lignifiés et subérifiés). L'examen par transparence, après aplalissement sur la lamelle, devra presque toujours être complété par l'examen des éléments histologiques, séparés par dissociation complète. Pour des tissus mous, presque uniquement formés d'éléments parenchymateux, le procédé de dissociation suivant est particulièrement recommandable. Les minces fragments de tissus à examiner sont mis à séjourner dans l'alcool, additionné d'un quart d'HCl. Après vingtquatre ou (quarante-huit heures, on lave soigneus ement, et à l'eau de lavage on ajoule un peu d'oxalate d'ammoniaque; par légère agitation la dissociation parfaite s'obtient et, dans le liquide trouble, nagent des cellules dissociées, des lambeaux d'épiderme et des fragments de faisceaux libéro-ligneux. L'ne filtration sur toile métallique de platine, à mailles très fines, retient les lambeaux d'épiderme et les faisceaux; le liquide trouble qui passe laisse déposer une pulpe, que l'on peut examiner directement ou après l'action de colorants.

b) Caractères histoloyiques. - Nous avons trop longuement insisté au chapitre I ${ }^{e r}$ sur les caractères histologiques des divers organes des Arum, susceptibles d'ètre rencontrés au cours d'une expertise pour y revenir ici.

Ces données sont complétées (p. 15ั6) par l'exposé des caractères histologiques de la feuille d'Useille, qui est susceptible, dans certains cas, de prêter à la confusion. En possession de ces données, un expert spécialiste pourra toujours, par un examen microscopique approfondi, arriver à des conclusions précises.

Quelques réflexions seront ici à leur place.

Dans les cas, fort rares apparemment, où le tubercule d'Arisarum v'ulyaie serail incriminé, les caractères spéciaux de son tubercule, en particulier, les formes caractéristiques des cristaux, presque 
inaltérables, d'oxalate de chaux qu’il contient, seront des éléments précis pour le différencier d'avec des débris de radis ou de navet.

Au cas où on ne posséderail que de menus débris de feuilles et de pétioles, la distinction entre les Aroüdées indigenes el les autres plantes vertes alimentaires, en particulier l'oseille, sera toujours possible, sinon facile. La présence abondante des laphides chez les Aroïdés, des mâcles en oursins chez l'oseille, serait un des caractères les plus précieux, mis surtout en évidence par l'examen des débris en lumière polarisée, où les cristaux s'éclairent de teintes vives, même s’ils sont disséminés en petit nombre dans les préparations.

Les caractères fournis par les cellules épidermiques, en particulier par les stomates, seront aussi des plus précieux, vu la cohésion des lambeaux épidermiques, et l’inaltérabilité assez grande de leur structure par la digestion, ou une putréfaction de moyenne durée.

Sans attacher une créance trop grande, surtout dans des recherches médico-légales, à des réactions de micro-chimie végétale, il nous semblerait bon, en cas d’intoxication par des baies d'Armm ou des organes végétaux riches en pigments rouges ou orangés, de terminer la détermination histologique de ces organes par une recherche microchimique, ayant pour but d'extraire et de caractériser ces pigments.

Ces pigments se rapportent vraisemblablement tous au groupe, encore vaguement défini au point de vue chimique, des pignents lutéiniques, dont le type le mieux connu est la carottine de la racine de carotte. Ces pigments offrent une résistance remarquable à l'action des liquides acides, et même de la putréfaction.

Le pigment des baies d'Arum élant fort peut altérable, l'examen microscopique des cellules du mésocarpe a toutes chances de révéler la présence des granulations amorphes, engagées dans les 
lenciles, el dont nous avons précédemment indirué les caractères. (V.p. 42.)

Nous nous sommes assuré, qu'en particulier, les baies d'Arum ne subissenl dans le tube digestif aucune altération appreciable de leur pigment. Il est possible de l'extraire, si ces baies ont été ingérées en quantitis suffisante,mème du contenu d'un estomac en putréfaction. Dans les matières vomies, leur recherche serait encore bien plus facile.

Pour semblable recherche, il nous parait falloir suivre, d'une manière générale, la marche suivante :

$1^{\circ}$ Rassembler par triage à la main ou à la pince, tous les débris épars colorés en rouge ou en orangé, les débarrasser par lavage à l'eau (les pigments lutéiniques sont insolubles dans l'eau), sur un filtre, ou sur un treillis en fil de fer à mailles fines, de toutes les particules étrangères à la pulpe de fruit, et des matières colorantes qui les souillent.

$2^{\circ}$ Procéder à l'extraction du pigment, à l'aide de dissolvants appropriés. La méthode tui a domné à Counchet de bons résultats, sur les tissus frais, est applicable, avec évidemment moins de chances de succès, à des tissus fragmentés et macérés. Voici cette méthode.

L'organe coloré est divisé aussi parfaitement que possible, ou mème réduit en pulpe, puis mis en macération dans l'alcool, l'éther, le chloroforme ou la benzine, après qu'on a éliminé avec soin toutes les parties qui pourraient introduire dans le dissolvant des matières colorantes étrangères. $A u$ bout de deux ou trois jours de macération à froid, Ia liqueur contient à peu près tout le pigment qu'elle est susceptible de dissoudre. Pour la concentrer, il suffit, si l'on s'est servi comme véhicule de l'éther ou du chloroforme, de l'abandonner à l'air dans un flacon à large ouverture, que l'on recouvre simplement d'une étofle légère ou d'un morceau de papier, pour empêcher la chute des poussières. Si l'on emploie la benzine ou l'alcool, il est nécessaire d'évaporer lentement au bain-marie. Mais, comme les matières colorantes sont en général très altérables, il est préférable d'employer, quand on le peut, le chloroforme ou l'éther, afin d'éviter l'action de la chaleur. Lorsque la concentration est assez avancée, on voit se former soit des globules rouges, qui se réunissent au fond du réservoir, soit des particules rouge-brique, qui se déposent également ou qui flottent dans la solution. On aspire alors à l'aide d'une pipette ces glo- 
bules ou ces particules colorés, et on les soumet à l'examen microscopique. On peut aussi examiner directement une solution concentrée, dans laquelle on voitse former des cristaux, si la liqueur est susceptible d'en produire. Ce dernier procédé toutefois, donne des résultats moins satisfaisants, i cause de lat tendance que présentent les dissolvants à fuser loin des bords du couvre-objet.

Dans cette recherche, deux cas peuvent se présenter:

$1^{\circ}$ On a pu obtenir le pigment cristallisé.

a) Il est alors facile de constater l'identîté de ces formes cristallines microscopiques, avec celles que nous avons indiquées pour le pigment eristallisé extrait des fruits frais.

b) De plus, on peut, jusqu'à un certain point, les comparer à celles des pigments rouges des autres végétaux vulgaires, susceptibles d'être ingérés (tomate, piment, courge, carolte, etc.). Les figures données par Counciet pourraient, en l'absence de documents plus complets sur cette question, servir de termes de comparaison. De cet examen comparatif, on pourrait conclure, avec beaucoup de vraisemblance, à l'identité des cristallites obtenus, avec ceux de l'Arum, ou avec ceux de telle ou telle autre plante.

Il importe, dans cette comparaison, de ne mettre en regard que des cristallites déposés au sein d'un même dissolvant, la nature du dissolvant pouvant influer sur la forme, souvent mal définie cristallographiquement, de ces cristaux microscopiques.

$2^{\circ}$ Le pigment est resté en solution dans les divers dissolvants employés, il se refuse à cristalliser.

Dans l'ćlat actuel de nos connaissances, plus qu'insuffisantes, sur les propriétés chimiques des pigments, nous ne pourrons tout au plus qu’invoquer quelques réactions, sensibles, et caractéristiques d'ailleurs. Elles ne pourront qu'indiquer que nous arons affaire à un pigment lutéinique.

Deux réactions sont à retenir; elles sont très sensibles et n’exigent que des quantités fort minimes de substance :

a. So $\mathrm{H}^{2}$ concentré colore en violet ou en bleu plus ou moins pur les solutions des pigments lutéiniques, et encore mieux les cristaux ou cristallites qu'elles laissent déposer; la teinte primitive 
passe rapidement au vert sale ou au brun, et le pigment est détruit. Cette réaction est fugace.

$b$. L'iode en teinture, ou, mieux encore, en solution dans l'iodure de potassium, les colore en bleu verdâtre ou en vert.

En résumé, dans les expertises relatives à une intoxication par les Aroïdées indigènes, la détermination botanique de la plante incriminée sera toujours possible, alors que la recherche chimique des principes toxiques n'est guère appelée à donner que des résultats douteux. 


\title{
DEUXIÈVE PARTIE
}

\section{REGHERGHES MÉDICALES SUR LE GENRE ACT ÆA L.}

\author{
CHAPITRE PREMIER
}

\section{ÉTUDE BOTANIQUE}

Le genre Actce $L$. (de actcia, en grec: sureau, à cause de la ressemblance du fruit de l'espèce indigène avec celui de cet arbuste) classé jadis dans les Rosacées (TourneforT) appartient, depuis Jussier, à la famille des Renonculacées, série des Clématidées pour les uns (Baillon), des Actæées pour les autres (Rocy et Fouc). (On ne sait pour quelle cause (quelques auteurs ont voulu en faire le type d'une famille spéciale des Actéacées.)

Deux espèces, dans ce genre, attireront seules notre attention, au point de vue médical : l'espèce indigène $A$. spicatu, comme plante réputée éminemment toxique el dangereuse, et l'espèce américaine, A. racemosa, comme médicament célèbre, d'un usage courant dans son pays d'origine, et d'autant plus digne d'être étudié comme tel, que son histoire chimique, physiologique et chimique, mérite d'être totalement reprise.

\section{A. - Actæa spicata L.}

Syn.

Actæa acuminala, WALL.

- arguta NutT.

- brachypetala var.a D.C. 
Actac erylhrocarpí Fisch.

- longipes SPACH.

- nigra GerTN.

- racemosa Gilib.

- Christophoriana spicata MoEnch.

vulgaris nostra racemosa et ramosa TounN.

Noms vulgaires: Actée en épi. - Actée des Alpes. - Christophoriane. - Christophorienne. - Herbe de Saint-Christophe. - Ilerbe aux poux.

\section{MODE DE VÉGETATION}

C'est une plante vivace, à tige souterraine, courte, trapue; qui émet chaque année une, ou plus rarement, un petit nombre de rameaux aériens. Pendant les premières années de végétation, ces rameaux se bornent à émettre des feuilles en petit nombre, souvent une seule. Alors même qu'il n'y a qu'un seul rameau foliifère, il existe toujours des rameaux dormants de remplacement, émis, soit par la propre base de ce rhizome, soit par celle du rameau aérien de l'année précédente. A la fin de sa première année de végétation, toutaxe aérien porte, à l'aisselle de ses cicatrices foliifères, de gros bourgeons latéraux, prêts à s'épanouir en pousses feuillées, l'année suivante, et un bourgeon terminal, d'abord abrité dans la gaine de la dernière feuille, entouré d'écailles d'un blanc rosé, et qui s'épanouira l'année suivante, en pousse foliifère. Ce n'est que.sur les pieds âgés que se développent des inflorescences, le plus souvent terminales.

Sur les pieds vigoureux, à l'aisselle des feuilles basilaires, peurent se développer, à l'automne, de petites inflorescenceslatérales, dont les fruits arrivent rarement à maturité.

Chaque rameau, qui a fleuri, disparaît jusqu'à sa-base; il est remplacé, au printemps suivant, par un autre rameau, émis aussi par le rhizome, ou par des bourgeons latéraux, émis par sa propre base persistante.

Ce que l’on décrit généralement, dans les flores, sous le nom de tige, n'est autre chose qu'un rameau aérien, émis par la vraie tige qui demeure toujours souterraine. 
L'Actée fleurit en juin-juillet, et ses fruits sont mùrs en août et septembre.

\section{$1^{\circ}$ Caractères organographiques.}

Rhizome. - Le rhizome, court, noirâtre, porte les cicatrices sigilliformes, peu proéminentes, des axes de végétation des années précédentes, détruits presque jusqu’à leur hase. Sur la base des vieux rameaux, et surtout de ceux qui fleuriront l'anncic suirante, se voient les cicatrices annulaires des feuilles disparues. De nombreuses racines adventives, plus ou moins abondamment ramifiées, sont émises par les bases des rameaux aériens, à mesure que les plus anciennes disparaissent sur les portions àgées du rhizome.

Les bourgeons dormants sont, à l'automne, gros, ovoïdes, protégés par des écailles, les extérieures brunes, les intérieures d'un blanc rosé.

Chaque rameau ciérien, ì axe court, porte 1, 2 ou 3 feuilles, alternes, grandes.

Feuilles. - Les feuilles sont complètes, largement engainantes à la base, à pétiole progressivementatténuévers le haut, bi-ou triternatiséquées, décomposées suivant le mode penné, à divisions ultimes tripartites.

Liexamen de la figure de ces feuilles ifig. 1, pl. 4) suffit mieux à graver leurs caractères dans la mémoire qu'une longue description (1).

Les folioles terminales ne sont portées que par des pétiolules courts, elles sont ovales ou oblongues, aiguës, incisées, dentées, les folioles latérales, nettement dissymétriques, à limbe rétréci du côté du rachis qui les porte.

Les feuilles diminuent nettement de taille, à mesure que l'on s'élève sur l'axe qui les porte. Les plus élevées sont réduites à

(1) Pour plus de précision, on peut dire :

Le pétiole de chaque feuille, après un léger renflement, se divise en 3 pétiolules primaires (l'un médian, les 2 autres latéraux) dépendant de 3 folioles primaires. Chaque pétiolule primaire se divise à son tour en 3 pétiolules secondaires, dépendant de 3 folioles secondaires; chacune de ces folioles secondaires se divise en 3 folioles tertiaires. Chacune de ces folioles ertiaires est plus ou moins nettement tripartite. 
leur gaine et ì leur limbe divisé ; finalement on passe à des loractées axillantes par rapport aux ramifications de l'inflorescence ou aux fleurs qui la constituent.

Inflorescence. - Les fleurs, petites, sont groupées en petite grappe simple, serrée, ovale dans l'ensemble, le plus souvent terminale. II y a, fréquemment, ure grappe àl'aisselle de toutes ou presque toutes les feuilles des rameaux aériens; 1 ğ grappes évoluent d’aulant plus lardivement qu'elles sont plus inférieures. Les grappes sulpérieures sont à l'aiselle des bractées. Il peut arriver que l'axe de la dernière grappe latérale soit concrescent à la base avec celui de la grappe qui termine le rameau, et fasse croire, alors, a un examen superficiel, à une ramification de celte grappe terminale. La concrescence peul, d'ailleurs, se produire aussi entre un pédoncule floral et l'axe sur lequel il s'insère.

Les fleurs, régulières, ont un réceptacle presque plan et un périanthe double. Les fleurs sont presque toujour's solitaires à l'aisselle des bractées alternos; jeunes, elles sont accompagnées de deux bractéoles, latérales, denticulées, caduques de bonne heure, dont l'une a une fleur à son aisselle.

Le.verticille extérieur du périanthe est formé de 3 à 5 pièces (sépales?) pétalö̈des, blanches, rougeâtres au bout, caduques de bonne heure; lorsqu'il y a 4 pièces, 2 sont extérieures et latérales, se recouvrant l'une l'autre, 2 intérieures; souvent, mais non toujours la postérieure recouvre l'antérieure.

Le verticille intérieur est formé de 4 à 10 pièces, petites, spatılées, onguiculées; dépourvues de tout nectaire à la base, regardées généralement comme des staminodes, alternant (souvent mais non loujours) exactement avec les sépales.

L'androcée forme, dans son ensemble, une sorte de houppe; il est composé de nombreuses ćtamines à filet libre, filiforme, à anthère basifixe, introrse.

La position du carpelle n'est pas constante; il est, tantôt superposé à un sépale, plus souvent alterne avec l'un d'eux. L'ovaire ovoïde, courtement stipité, aplati sur sa face axile (parcourue par un raphé correspondant auplacenta), est surmonté d'un stigmate ì 


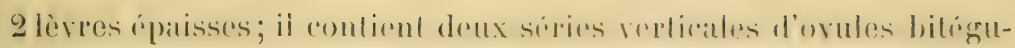
mentés. Les premiers-nés apparaissent à mi-hauleur du placenta, les autres apparaissent successivement vers le bas et vers le haut.

Les baies de la grosseur d'une belle groseille à cassis, à malurilé, sont ovoüdes, aplaties sur leur face axile, à raphé encore bien visible sous forme d'un sillon longitudinal, surmontées du reste puncliforme du stigmate, le pédoncule a une insertion nettement excentrique. Le péricarpe charnu laisse échapper ì la pression un suc pourpré, et ne s'ouvre pas pour laisser échapper les graines.

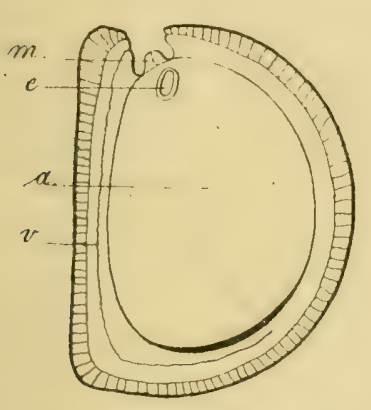

Fig. 4\%. Actrea spicata L. (Gross. 8/1). Graine, c. longitudinale médiane. $m$ imicropyle, $e$ embryon, $a$ albumen, $v$ vaisseaux du funicule.

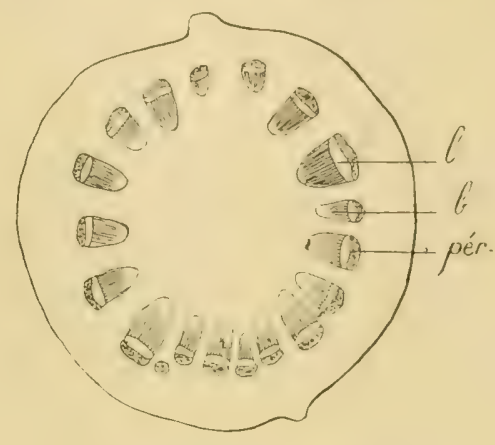

Fig. 45. Actra spicata L. (Gross. 8/1). Rhizome, port. jeune, c. transvers.) (semi-schèma.). $l$ liber, $b$ bois, per péricycle.

La surface des graines, lisse en apparence, est hérissée de petites lamelles peu saillantes, et formant par leur ensemble, vues à un faible grossissement, une sorte de petit réseau.

Les graines affectenteux formes différentes, selon qu'elles oceupent le milieu ou les extrémilés de la cavilé du fruit; elles sont égallement aplaties sur leurs fatces (supérieure et inférieure pour la šrainéen place), et affectent prestue la formo de demi-disques, à converité externe; leur face interne 'st parcourue par un étroil sillon, limite par deux lèvres saillantes, et qui correspond au raphé. Elles renferment, sous un double tégument, un albumen abondant, amylace, renfermant un très petil embrỵon, à peine diflérencié, rentli, subsphérique, dicotylé. 
Les dimensions des organes sont :

Pédoncule floral............... longueur $0,6^{\mathrm{mm}}$ (ch. moy.)

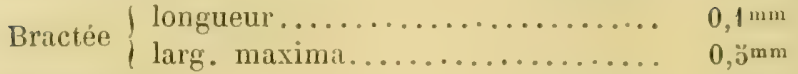

Bractéoles................. longueur $0,5 \mathrm{~mm}$

Fleur (lors de l'anthèse):

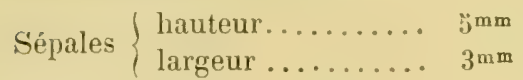

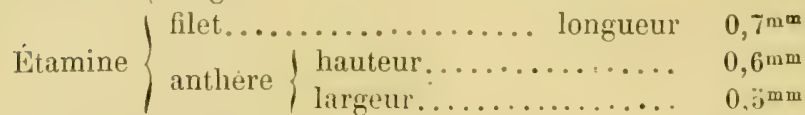

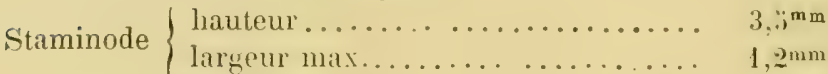

Ovaire $\left\{\begin{array}{l}\text { hauteur } \ldots \ldots \ldots \ldots \ldots \ldots \ldots \ldots \ldots \ldots \\ \text { largeur. } \ldots \ldots \ldots \ldots \ldots \ldots \ldots \ldots \ldots\end{array}\right.$

Fruit mûr $\quad\left\{\right.$ hauteur. . $\quad \ldots \ldots \ldots \ldots \ldots 12^{\text {nm }}$

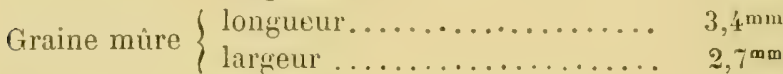

Répartition géographique. - A. spicata est surtout une plante des localités fraîches, montueuses et boisées. On la trouve aux environs de Paris, mais plus fréquemment dans le nord et l'est de la France (elle

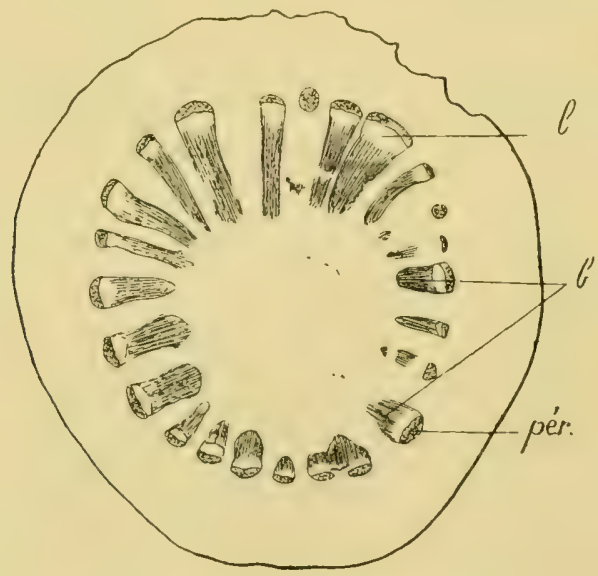

Fig. 46. Actæa spicata L. (Gross. 8/1). - Rhizome (port. âgée) c. transvers. (semi-schéma).

n'est jamais très commune dans les localités où on la rencontre); fréquente dans les Alpes, l'Auvergne, le Forez, les Corbières, elle est rare dans le 
Centre, les Pyrénées, le Midi, et on ne rencontre pas dans l'Ouest (de l'Orne ì l'Océan), ni en Corse.

Elle s'étend à l'Europe septentrionale et centrale, l'Espagne, l'Italie continentale, la Dalmatie, la Bosnie, la Serbie, le Monténégro, jusq̣u'au Caucase, en Sibérie et dans l'Inde.

Les variétés melanocarpa LEDEB., erythrocarpa TURCz, et leucocarpa LEDEB., signalées daus le nord de l'Lurope et en Sibérie, ne se rencontrent pas en France.

\section{$2^{\circ}$ Caractères anatomiques (1).}

Rhizome. - Selon le niveau où on l'examine, on voit sur la coupe transversale la trace de linsertion des écailles protectrices des jeunes pousses. La coupe transversale, régulièrement circulaire, montre un cercle unique de 18 faisceaux fibro-vasculaires environ, ¿̀ section cunéiforme, plus allongés sur le rhizome ìgé, et aux points où ils fournissent aux nervures des écailles.

La coupe transversale du rhizome àgé montre, en allant de dehors en dedans :

Épiderme, exfolié par places, à paroi externe épaissie, mais non lignifiée, à cavité souvent occupée par des expansions, en doigt de gant, des parois.

Exoderme, nullement distinct du parenchyme cortical, formé d'élémentsréguliers, ovales, à parois médiocrementépaissies, d'apparence subcollenchỵmateuse, séparés par des méats petits et nombreux, à cavilé souvent parcourue par une expansion giêle, rísultant de la rupture d'une partie de la paroi.

Des ares péricycliques, fibreux, non lignifiés, sont superposés au dos des faisceaux fibro-vasculaires.

Chacun de ces faisceaux est formé d'un massif liberien, olsquadrangulaire, ou quelque peu allongré, et d'un coin ligneux, allongé vers le centre. Le bois est formé de vaisseaux, de médiocre diamètre, à section polygonale, agencés par petits groupes, irrégulièrement ordonnés eux-mêmes en files radiales, el de parenchỵme ligneux, à éléments polygonaux, à parois épaisses. Aucun de ces

(1) Aucune étude d'ensemble des caractères anatomiques d'A. spicata n'a encore été faite. On ne trouve que quelques détails épars dans les travaux de Marué et de Garcis. 
éléments du bois n’est lignifié. La pointe obtuse de chaque coin ligncux est occupée par un ilot de parenchyme, it petits éléments,

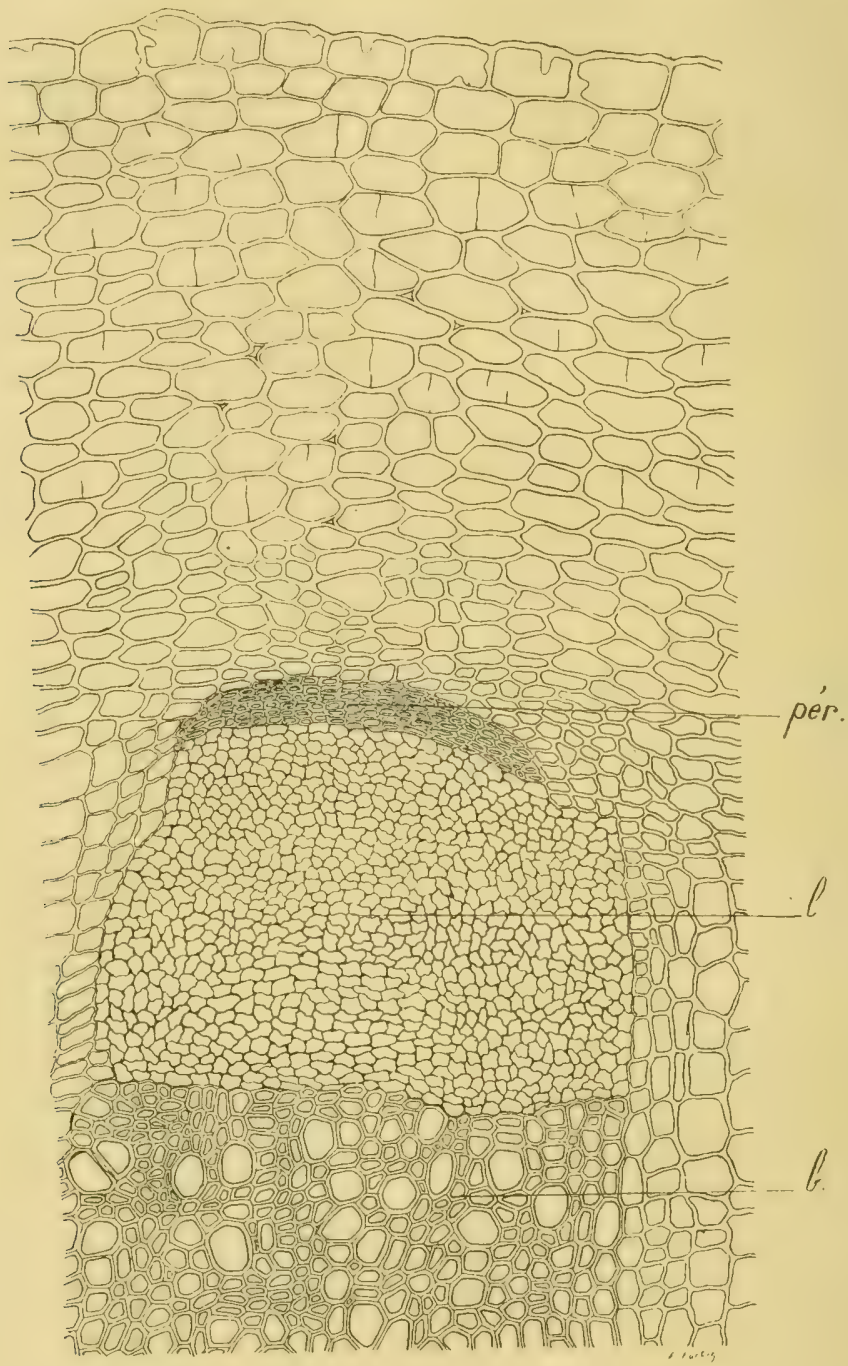

Fig. 47. Actra spicata L. (Gross. 120/1) - Rhizome (port. âgée), c. transvers. (scmi-schéma.). Port. périphérique. $l$ liber, $b$ bois, per péricycle, $r m$ rayons médullaires, $m$ moelle.

munis de parois minces, contenant épars les plus anciens vaisseaux. Rayons médullaires formés d'éléments parenchymateux, allongés, 
et alignés en files radiales, avec quelques miats. Noclle à éléments ovales, réguliers, à paroi médiocrement ipaissie, ponctuée, avec nombreux méats.

Racine. $-1^{\circ}$ Structure primaire. - Une jeune radicelle présente,

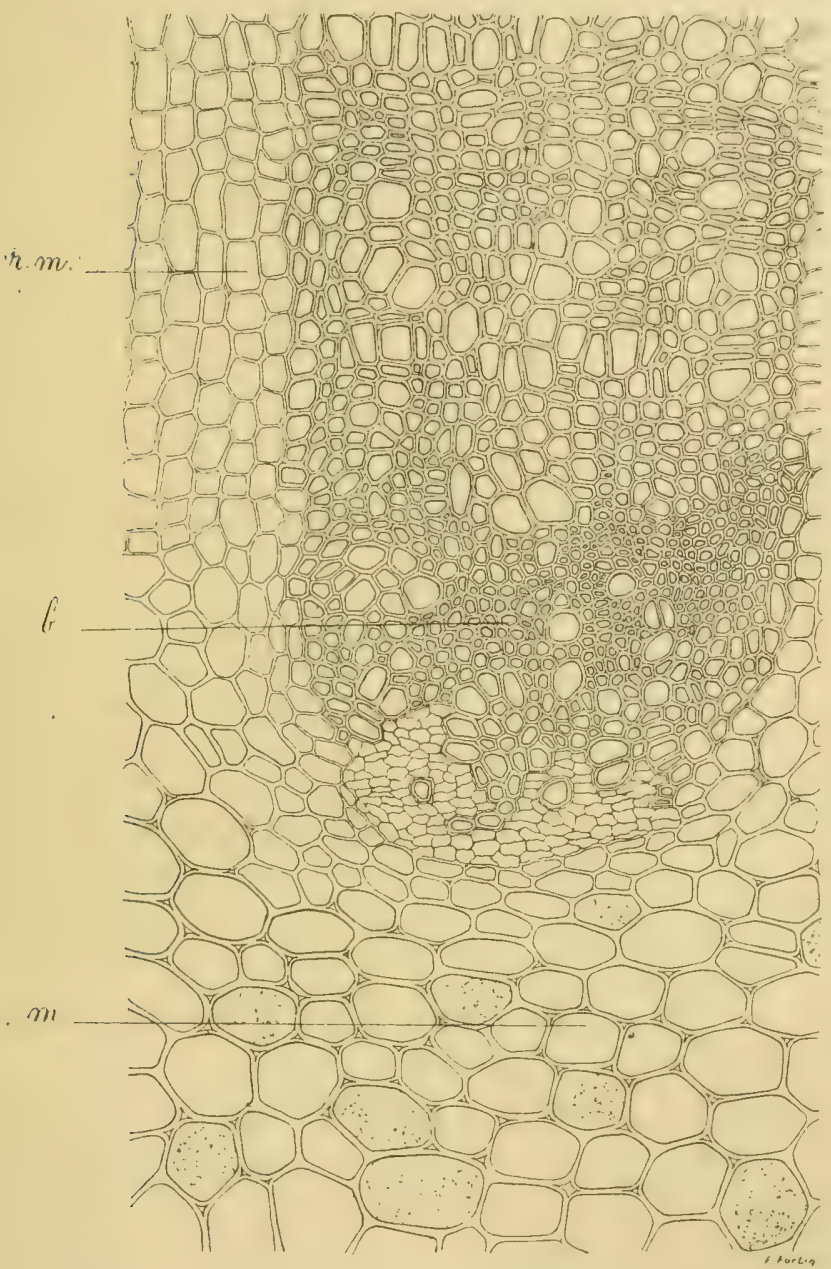

Fig. 48. Actæa spicata L. (Gross. 120/1). - Rhizome (port. âgée), c. transvers., (semi-schéma). Portion centrale. $l$ liber, $b$ bois, per péricycle, rm rayon médullaires, $m$ moelle.

en allant de la péripherie vers le centre /c. à la limite supérieure de la région pilifère) : 
Assise péripheirique, à éléments quelque peu inégaux, à paroi cxtirieure saillante, très épaissie, non lignifiée. Cà et là, certains éléments se prolongent encore en poils absorbants.

Parenchyme cortical formé. d'assises circulaires, à éléments régulièrement alternes, de diamètre d'autant plus considérable que l'on serapproche davanlage du centre, à paroi épaissie, collen-

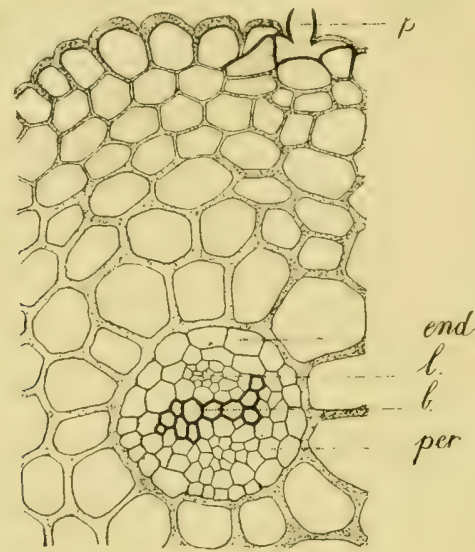

Fig. 49. Actæa spicata L. (Gross. 180/1). - Radicelle adventive (struct. prim.).

chymateuse; endoderme net, mais sans cadres d'épaississement.

Péricycle à peine distinct durestedu conjonctif central.

Deux, trois ou quatre faisceaux libériens, alternant avec autant de faisceaux ligneux réunis au centre. Parenchyme médullaire très réduit.

$2^{\circ}$ Structure secondaire. - Une radicelle adventive âgée possède :

Parenchyme cortical à parois plus minces.

Endoderme et péricycle à peine distincts du parenchyme des rayons médullaires et de la moelle. Les faisceaux ligneux primaires restent bien visibles, sous la forme de coins nets, mais peu saillants, dans la concavité des ares limités par les faisceaux ligneux secondaires, différenciés à la face interne des faisceaux libériens primaires. Vaisseaux du bois secondaire nombreux et larges, à sertion subcirculaire, parenchyme ligneux à éléments polygonaux, à paroi épaisse, non lignifiée. 
Moelle à éléments réguliers, polygonaux ì parois minces.

Rameau cérien. - La base (souterraine d'ailleurs) des rameaux aériens diffère à peine, par sil structure, du rhizomm. L'épiderme y a

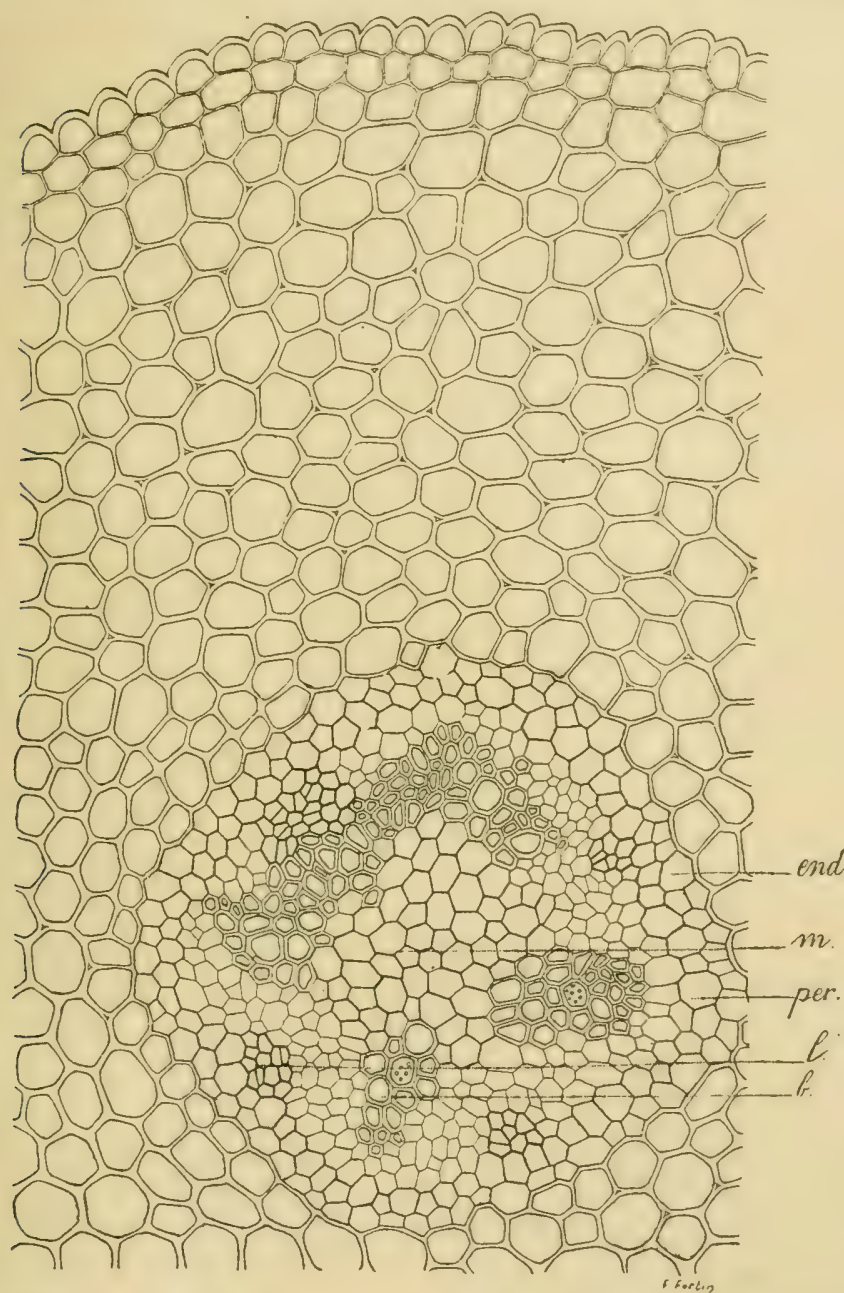

Fig. 50. Actra spicata L. (Gross. 120/1). - Radicelle adventive (passage à la struct. second.)

une paroi externe plus mince; les faisceaux sont plus ramassés, non allongés dans le sens radial, ont it leur dos un are fibreux péricyclique, incomplètement lignifié, et le centre de la moelle a une ten- 
dance à se désagréger pour rendre la base du rameau fistuleuse.

La partie supérieure (subaérienne) du rameau présente:

Epiderme à paroi externe, épaissie, faiblement lignifiée, parcourue de petites crêtes longitudinales. faiblement saillantes;

Exoderme, en rien distinct du reste du

Parenchyme cortical formé d'éléments très réguliers, ovales, de taille inegale, à parois médiocrementépaisses, séparés par desméats;

Une zone péricyclique continue, formée d'éléments de taille inésale, obcirculaires, a parois très épaissies, non lignnifiées, séparés par de petits méats triangulaires; cette zone, réduite au dos des faisceaux à une seule couche de cellules, s'épaissit fortement, en face des rayons médullaires.

Les faisceaux présentent les mêmes caractères qu'à la base du rameau.

Moelle homogène, non fistuleuse.

Pétiole (avant sa division en pétiolules).

C. initiule obovale, aplatie à la face supérieure, les faisceaux s'y disposent en un cercle régulier périphérique, suivant le contour extérieur, la partie médullaire est irrégulièrement fistuleuse.

$C$. médiane triangulaire, à base supérieure, à angles arrondis, même disposition des faisceaux, moelle largement fistuleuse.

$C$. caractéristique (au niveau du renflement de la trifurcation) même forme qu'à la médiane, mais plus large el aplatie.

A tous les niveaux, la disposition régulière des faisceaux à la périphérie, parallèlement au contour extérieur, persiste.

Un faisceau médian inférieur, 2 latéro-supérieurs, et 2 médians supérieurs presque juxtaposés, font saillie vers le centre, à cause de leurs plus fortes dimensions.

Les caractères histologiques sont ceux du rameau aérien, à quelques différences près :

L'assise péricyclique y est un peu moins épaisse en face des rayons médullaires, les coins ligneux de dimensions plus réduites. Les faisceaux principaux, riches surtout en éléments vasculaires, ont leur pointe plongeant dans une plage de parenchyme à éléments petits, à paroi mince, bien distincts du parenchyme médul- 
laire, à élements plus larges, réguliers, polygonaux, à paroi mince, séparés par des méals. Toute la région médullaire centrale a ses éléments tassés, irrégulièrement aplatis et rompus, limitant la cavité résultant de la dislocation du tissu médullaire.

Limbe des folioles terminales. - Nervure médiane (G. au milieu) convexo-concave, un peu irrégulière, avec 4 faisceaux.

Épiderme à paroi uniformément épaissi , l’extérienrestrier', non lignifiée.

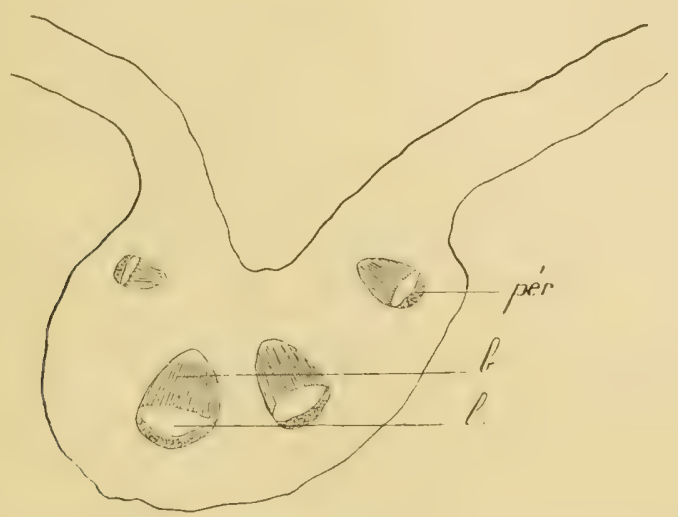

Fig. 51. Actra spicala L. (Gross. 40/1).

Nervure médiane d'une foliole terminale, c. transvers. (semi-schéma).

Hypoderme nettement collenchymateux.

Pas d'assise péricyelique continue, mais des ares péricycliques, au dos des faisceaux, formés de 2-3 couches de cellules, à parois épaissies, non lignifiées. Coins ligneux, de dimensions réduites, pauvres en parenchyme.

Limbe. - Épiderme supérieur à éléments irréguliers, de taille inégale, onduleux, allongés au-dessus du trajet des nervures, à section olsectangulaire, irrégulièrement saillants rers l'extérieur, à cuticule très finement striée, à parois minces, avec épaississements punctiformes, dépourvu de stomates.

Épiderme inférieur, présentant les mêmes caractères, ì éléments plus aplatis, it cuticule plus nettement striée, avec nombreux stomates. Ceux-ci sont ovales, situés sur un plan inférieur par 
papport à celui de l'épiderme, avec chambre susostiolaire, limitée par les bords ondulés des cellules annexes.

Mésophylle presque homogène, sans assise palissadique nettement différenciée, très irrégulièremert lacuneux, arec chambres
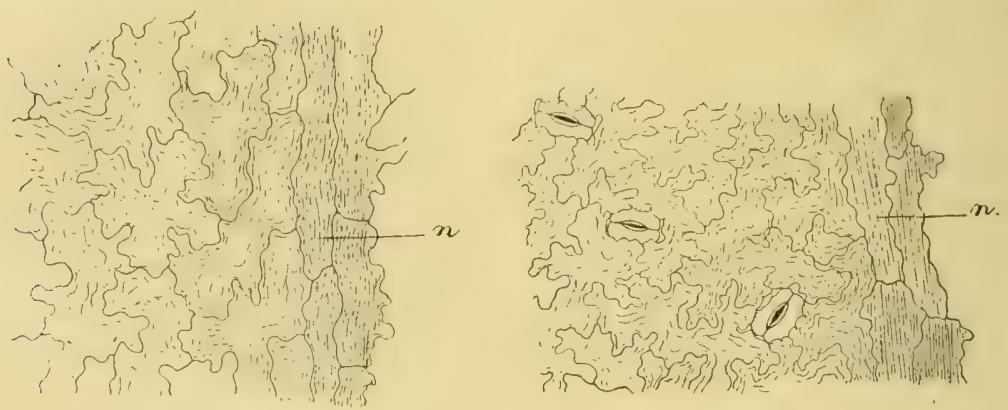

Fi g. 52. Actæa spicata L. (Gross. 120/1). Fig. 53. Aclæa spicata L.(Gross. 120/1).

Limbe d'une foliole. Epid. supér. Limbe d'une foliole. Epid. infér.) $n$ port. superposée à une nervure.

sus-stomatiques, dépourvu de tout élément de soutien différencié. Nervures quelque peu saillantes à la face inférieure, isolées dans le mésophỵlle, à peu près à égale distance des deux faces, entourées d'une zone protectrice de parenchỵme condensé, s'étendant jusqu'au contact des deux épidermes, à endoderme formé de cellules plus grosses que celles du péricycle.

Pédoncule du fruit. - Coupe tranvers., circulaire, à contour sinueux.
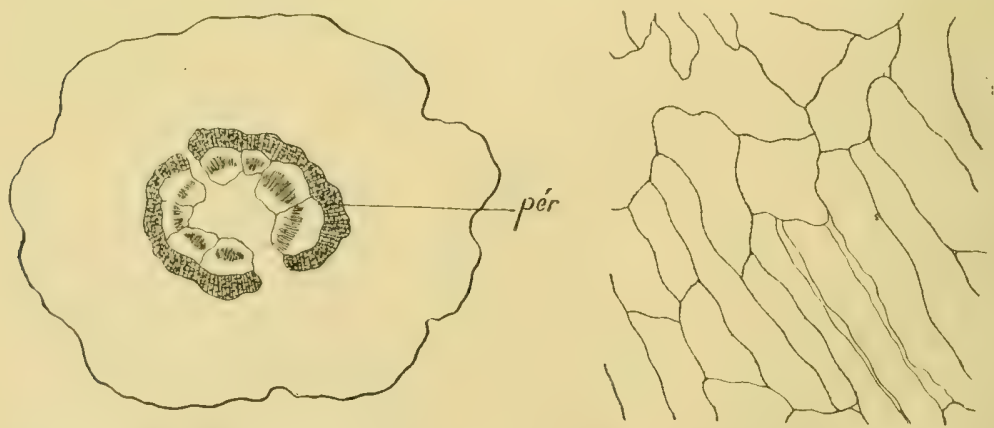

Fig. 54. Aclea spicata L. (Gross. 40/!). Pédoncule du fruit (c. transv.) (semischém.).

Fig. 55. Actra spicata L. (Gr. 120/1). Endocarpe. 
Épiderme à éléments quelque peu irrózuliers, à parois latérales souvent pliées, à paroi externe peu ipaissie, à cuticule finement et nettement striée. Quelques-unes des rellules épirlermiques se pro-
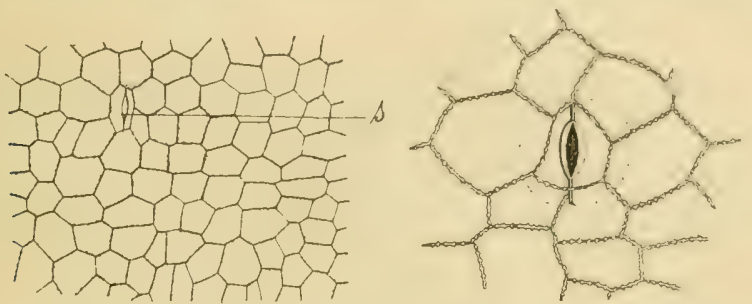

Fig. 5̋. Actæa spicala L. Epicarpe. - (1 Gross. 120/1) - (2 Gross. 260/1).

longent en poils cylindriques, appliqués, à sommet oblus, a paroi mince granulée. Parenchyme cortical à éléments assez réguliers, ovales, allongés et alignés en file radiale, séparés par des méats irréguliers, à paroi mince, onduleuse.

Faisceaux fibro-vasculaires groupés sur deux arcs en regard;

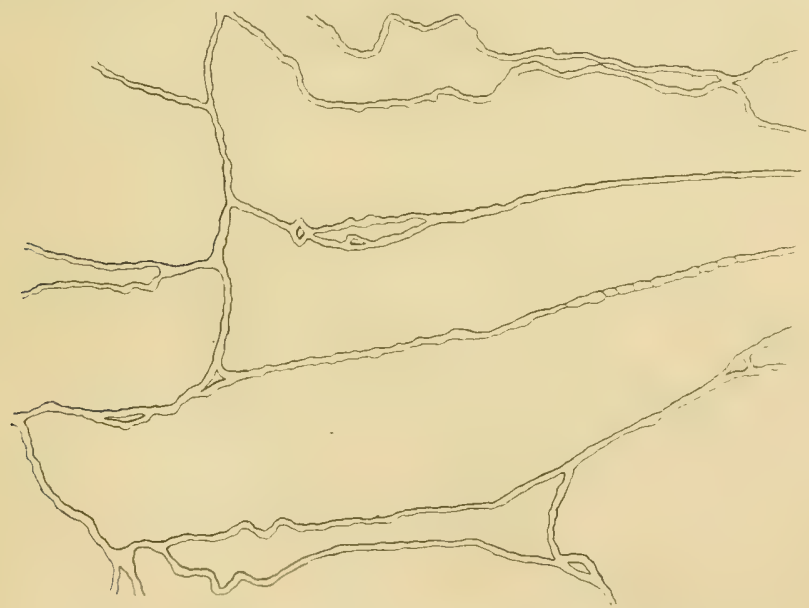

Fig. 57. Actæa spicata L. (Gross. 340/1). - Endocarpe.

avec un arc péricyclique d'éléments réguliers, à section circulaire, séparés par des méats, ì parois épaisses et collenchymateuses. Bois reduit à des éléments vasculaires, épars dans un parenchyme, à section circulaire, de petit calibre, non lignifiés. 
Moelle réduite, formẻe d'éléments réguliers, à section circulaire, séparés par des mẻats, à paroi épaisse, collenchymateuse.

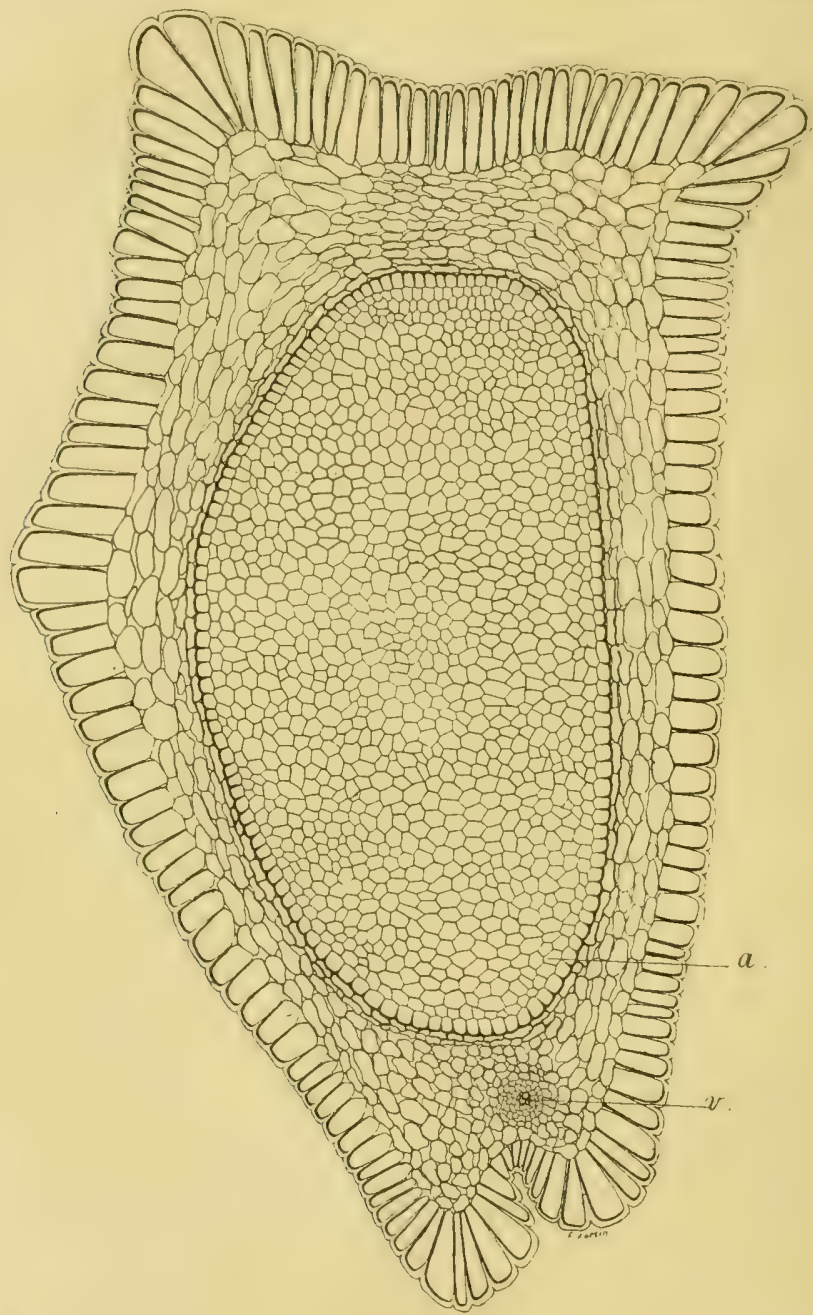

Fig. 58. Actrea spicata L: (Gross. 40/1), - v vaisseaux du funicule, a albumen.

Le péricarpe mùr est constitué par : un épiderme externe (épicallpe) à crllules labulaires (en c.), a parois langentielles, épaissies par de potites eranulations, polygonales (v. de litee), arec quelques 
rares slomales, un hypoderme d'une seule assise de cellules collenchymateuses.

Une chair formée de grands éléments sphéroïdaux ou comprimés.

Un épiderme interne (endocarpe), à cellules réticulées sur leurs laces rándiales, inégales, plus ou moins allongées (v. de face), à paroi irrégulièrement épaissie, souvent séparée sur une portion plus ou moins notable de sa longueur, de la paroi des cellules voisines.

Graine (mûre). - Les graines présentent, sur une coupe transversale pratiquée en leur milieu, une forme triangulaire ou pentagonale, irrégulière, le sommet du triangle correspondant au raphé, qui se dessine sous forme d'un sillon superficiel et étroil.

L'examen des coupes transversales et longitudinales permet de se rendre mieux compte, que par une longue description, Fir 59. - Actæa spicala L. (Gross. 40/1). des rapports des diverses couches qui sont:

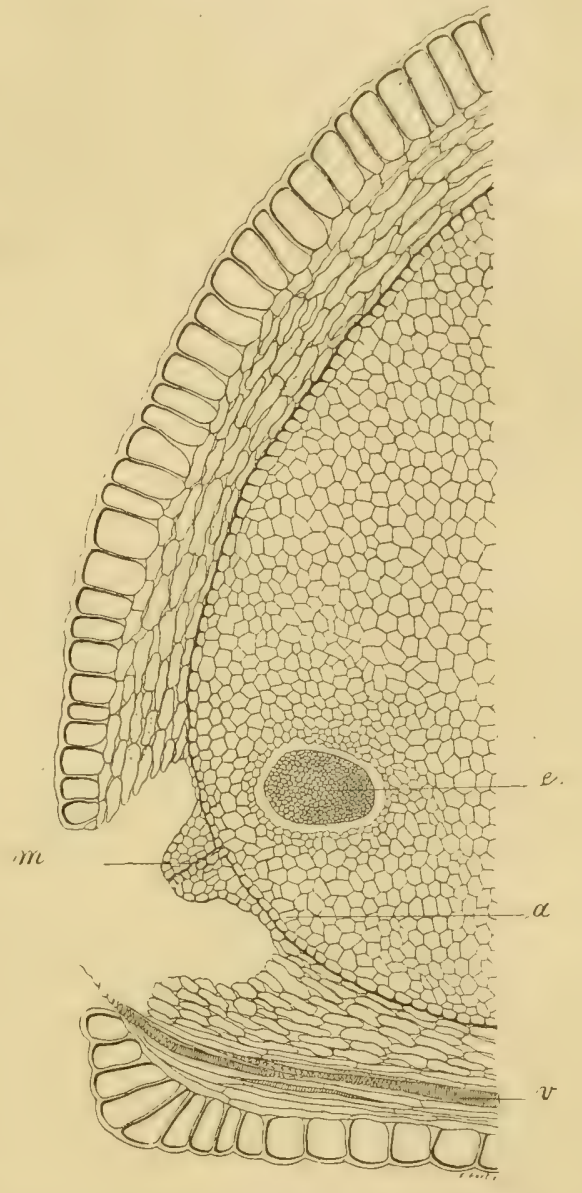

Graine (c. longitud. médiane). a albumen, $e$ embryon, $m$ micropyle interne, $v$ vaisseaux du funicule.

Deux téguments : l'externe à cellules allongées, dont les parois externes et radiales sont fortement épaissies et lignifiées; l'interne formée de ö-1; couches d'éléments assez réguliers, plus ou moins aplatis, à parois minces, laissant entre eux des méats 
nombreux "t irriguliers; la couche la plus interne de ce tégument affecte plus nettement la forme d'une assise tabulaire à petits éléments. C'est dans ce tégument que se trouvent les trachées du raphé, au nómbre de 3-4.

Un albumen rentral, homogène, amylacé, formé de petits éléments polygonaux, intimement pressés, dont l'assise périphérique est nettement différenciée en assise protectrice.

L'embryon, encore non différencié, a déjà provoqué, par digestion, la destruction des couches d'albumen, immédiatement en contact avec lui.

Il est facile de voir, sur la coupe longitudinale, exactement médiane, le micropyle interne persistant, avec son court et étroit canal.

Explicat. de la pl. IV.

\section{Actæa spicata L.}

GROSS.

FrG. 1. - Port .

2. - Inflorescence................................

3. - Fleur isolée.

4. $-\quad$ U. longit............................

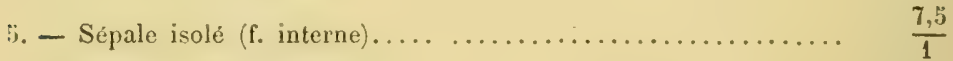

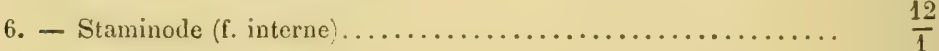

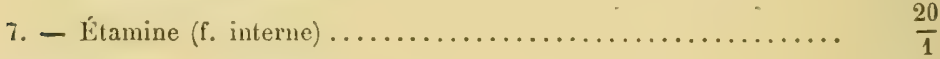

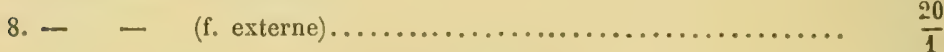

$9-9$ bis. - Ovaire (f. ventrale et $\mathrm{f}$. latérale)................. $\frac{6}{1}$

10. - Ovaire ouvert pour montrer les ovules................. 6

11. $-\quad$ C. longitud........................... $\frac{6}{1}$

12. - Jeune fruit (f. ventrale) ..................... $\frac{2,5}{1}$

13. - Fruit à maturité $($ f. ventrale $) \ldots \ldots \ldots \ldots \ldots \ldots \ldots \ldots \ldots \ldots \ldots \ldots \ldots \ldots \ldots \ldots, \frac{2,5}{1}$

14. $\quad$ - $\quad$ vu de $3 / 4$ (f. latérale) $\ldots \ldots \ldots \ldots \ldots \ldots \ldots \ldots \ldots \ldots \ldots \ldots$ 

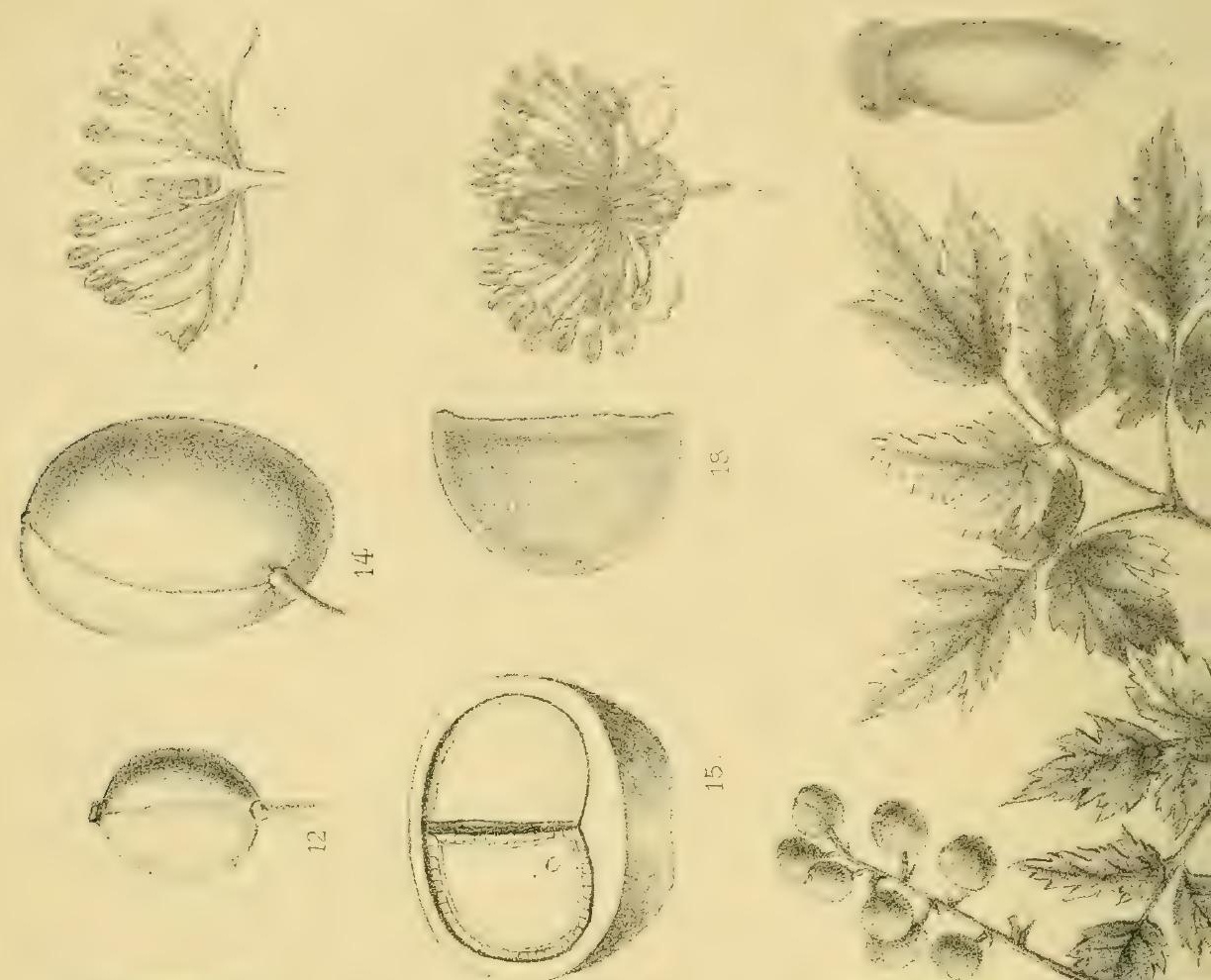

in
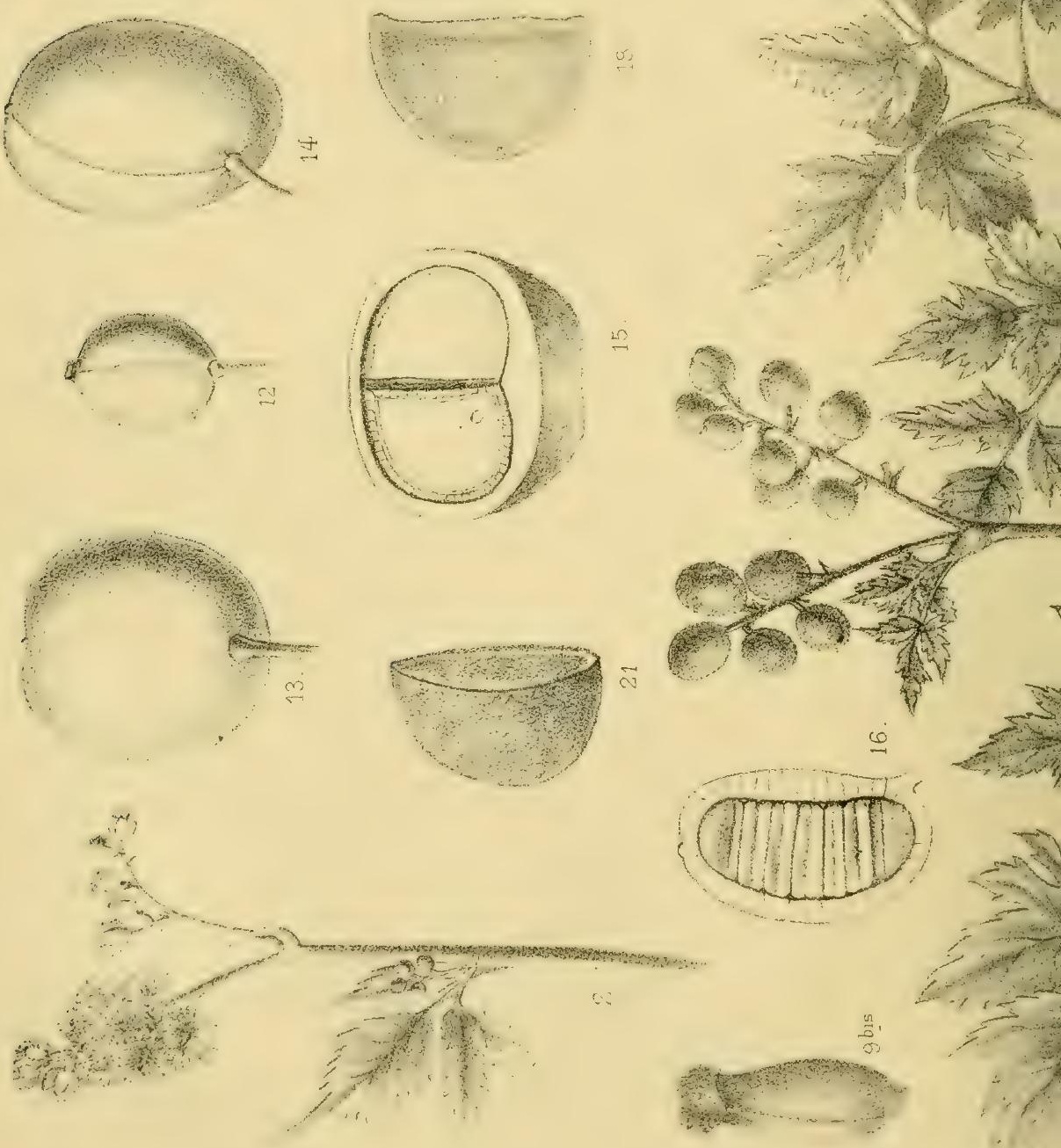



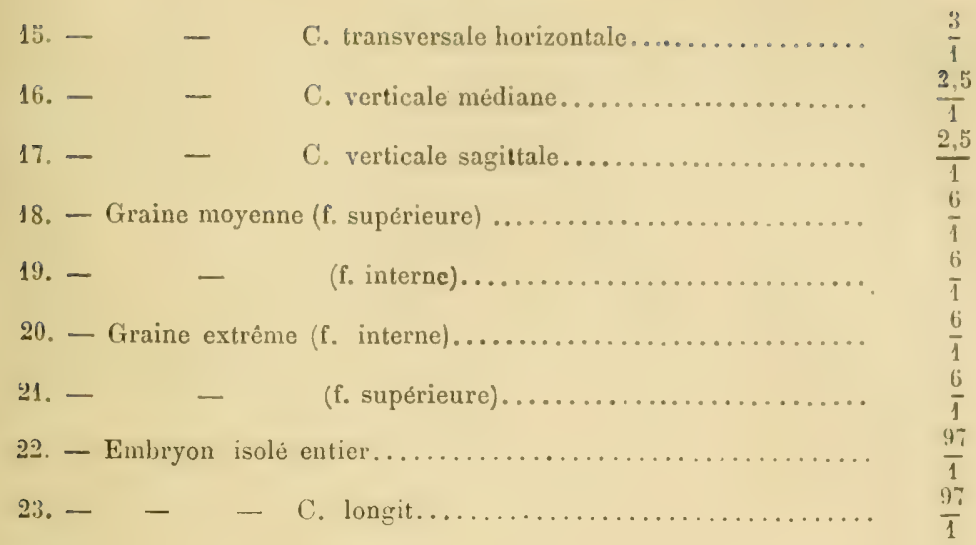

\section{II. - ACTEA RACEMOSA L.}

Syn. Actæa, monogyna IVALT:

Cimicifuga racemosa Elliott-BART.

- serpentaria Prrsh.

- orthostachya WENDER.

Macrotys (1) actroïdes RAFIN.

Noms vulgaires . - Actée en grappe d'Amérique. - Black Cohosh. Black Snakeroot. - Bugbane.

Nota. - Il importe de ne pas confondre cette plante avec d'autres espèces du même genre, désignées par certains auteurs sous la même dénomination spécifique. Les confusions les plus regrettables, au sujet de leur composition chimique et de leurs propriétés, pourraient ainsi s'établir :

Actæa racemosa GENERS. = Actra (Cimicifuga) fœtida $\mathrm{L}$.

- racemosa GiLis. = "spicata $\mathrm{L}$.

- racemosa WALT. = Trautvetteria palmata Fisch.

\section{$1^{\circ}$ Caractères organographiques.}

La grande analogie de bien des caractères de l'A. racemosa arec ceux offerts par l'A. spicate nous permettra d'ètre bref dans sa description. Le médecin n'est d'ailleurs pas intéressé directement à

(1) Nec Macrotys Rarin, ex. D. C. Syst. 
connaitre le détail de ses caractères orgamographiques, puisqu'il s'agit d'une plante exotique.

Mode de végétation. - C'est une herbe vivace, dont le mode de végétation est celui de l'A. spicata. Elle fleurit en juin et juillet, ses fruits mùrissent à l'automne, dans nos jardins botaniques où elle est fréquemment cultivée.

Rhizome. - Le rhizome est épais, horizontal, émeltant par sa face inférieure de nombreuses racines adventives, longues, cylindriques, plus ou moins ramifiées. Il émet, chaque année, une ou plusieurs pousses vertes, et se montre toujours richement pourvu de bourgeons dormants, recouverts d'écailles : les extérieures brunes, les intéricures d'un blanc rosé. Les cicatrices annulaires, laissées par ces écailles sur la base des axes aériens, sont nettement apparentes.

Chaque rameau aérien porte de 2 à 3 feuilles complètes, alternes, grandes, à pétiole engainant à la base. Le mode de partition du limbe est celui décrit à propos de l'A. spicata; mais, au lieu d'être réduit ì 3 folioles primaires, il comprend, implantées sur le rachis, suivant le mode penné, 2-3-4 folioles primaires, les inférieures triséquées, les supérieures souvent entières; les folioles terminales sont en général (sauf arortement plus ou moins complet des lobes latéraux) tripartites, toutes ovales oblongues, aiguës, inciséesdentées.

Les feuilles diminuent de taille, à mesure que l'on s'élère sur le rameau qui les porte, les supérieures sont réduites à leur gaine, et à leur limbe à 3 folioles primaires.

Infloresctnce. - Nous n'avons, sur les pieds cultivés au Jardin de la Faculté, jamais vu qu'une inflorescence terminale (les pieds cultivés dans les jardins botaniques doivent alteindre une taille très inférieure à celle qu'ils atteignent dans leur pays), et nous avons vu, chez l'A. spicata, que seuls les pieds vigoureux présentent des inflorescences latérales), en grappes composées au $2^{e}$ degré.

Les fleurs évoluent de la base de la grappe au sommet, de sorte que les plus inférieures sont déjà fructifiées, lorsque les supérieures ne sont encore qu'à l'état de boutons. 
Les fteurs, petiles, sont groupées sur l'axe de lat grappe allongie, solitaires à l'aisselle d' une bractée réduite, aiğü̈, courtenent pédonculées et accompagnées de deux bracténles latérales aiguës, le plus souvent stériles, caduques. Le réceptache, sensibl('ment plan, porte ^-̋ sépales inégaux, imbriqués dans le bouton, peitaloüdes, ovales, arrondis, d'un blanc jaunatre, cadues de bonne heure; un nombre irrégulier, généralement très réduit, de petits staminodes, cachés derrière les filets staminaux, courts, portis par un petit onglet, ¿̀ limbe lyré, bifide au sommet; des étamines nombreuses, a filet libre, allongé, filiforme, dilaté au sommet, à insertion basifixe, à anthère arrondie, à 2 loges séparées, à déhiscence latérale, d'un blanc crème pâle.

Le gynécée est réduit à un très petit nombre (souvent un seul) de carpelles obpyramidaux, à raphé tourné vers l'extérieur, renflés du côté externe, à sommet obtus, stigmatifère. La cavité unique de chaque ovaire porte, inséré sur presque toute la hauteur de sa face axile, un placenta muni de deux rangées verticales d'ovules (10 environ), anatropes, horizontaux, à micropyle extérieur, se regardant par leur raphé.

Le fruit, entièrement sec, est un follicule ovoüde, plus ou moins allongé, comprimé latéralement, à sommet légèrement incurvé, d'un vert pâle, déhiscent à partir du sommet, parcouru par des nervures saillantes horizontales, émergeant du raphé, et subdivisées.

Les graines présentent la plus grande analogie de forme avec celles de l'A. spicata, mais leur surface est finement villeuse.

Les dimensions moyennes des organes sont :

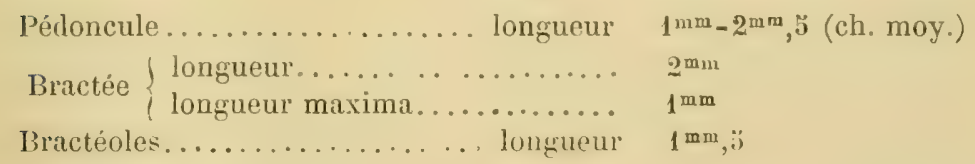

Fleur (lors de l'anthèse).

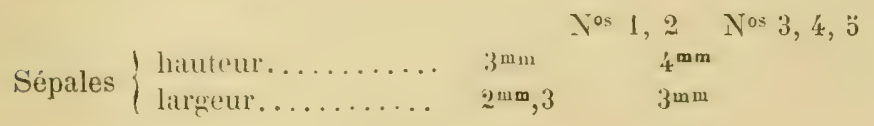




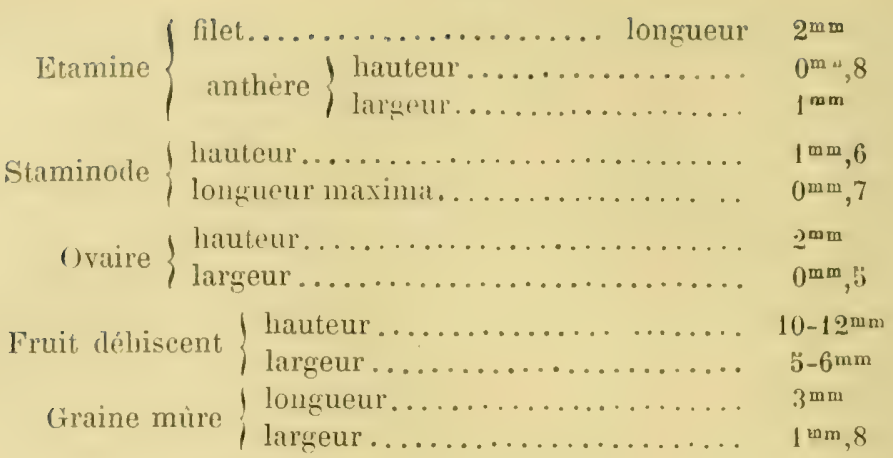

Répurt. géographique. - L'Actéa racemosı est indigène au Canada, aux États-Unis et jusqu'à la Floride; elle y croit abondamment dans les forèts, surtout dans certaines régions de la Garoline et de la Géorgie.

La plupart des auteurs classent l'A. racemosa dans un genre spécial, bien que distinct: Cimicifuga. C'est sous ce nom qu'elle se trouve le plus souvent désignée.

Les caractères distinctifs de ce genre: forme des staminodes, déhiscence du fruit sec, graines villeuses, ne semblent pas imposer la nécessité de cette distinction, et on peut laisser l'A. racemosa dans le genre Actra, dans une section spéciale: Cimicifuga (1).

\section{ÉTUDE PHARMACOGNOSIQUE DU RHIZOME}

\section{$1^{\circ}$ Caractères extérieurs.}

Rhizome. - Le rhizome, tel qu'il se trouve dans les drogueries, d'une odeur sui generis qui disparait en partie par la dessiccation prolongée, se présente sous la forme de fragments, d'un brun foncé, de saveur amère et âcre, astringente, irrégulières (ils ont été

(1) Il est, peut-être, aussi peu-naturel de séparer les Cimicifuga des Actra, par la déhiscence du fruit, que de séparer, pour la même raison, les Euthyra (Paris polyphylla) des Paris.

Un péricarpe, d'abord épais et charnu, peut perdre assez de sucs pour s'ouvrir à la façon d'une capsule, exemple: Lomatophyllum chez les Liliacées.

Pour grouper les Liliacées selon leurs affinités naturelles, il est indispensable d'admettre, dans certaines séries, des genres à fruit charnu et des genres à fruit sec. Il semble naturel d'agir de même pour les Renonculacées. 
coupés de diverses façons dans le rhizome), courts, noueux, plus ou moins ramifiés, de 3 à ä centimètres de long, en moyenne, d'un diamitre moyen de 23 millimitres. Le rhizoms, porle a sa fatce supérieure, les restes de plusieurs lamraux aériensasser largers, el, à sa face inférieure, des débris de racines tordues, cassées, dont il ne reste parfois que des cicatrices. Sa cassure est fibreuse. Sa section transversale montre, à l'aeil nu, une: zone perripherique subéreuse, peu épaisse; une zone ligneuse, bien visible, grâce aux faisceaux dont elle est formée, faisceaux cunéiformes, à pointe interne, plus ou moins larges, tranchant, par leur leinle blanchatre, sur le reste du parenchyme de teinte brunâtre; une zone médullaire assez réduite, de teinte brunâtre pâle.

Les racines adventives, friables, oltusément quadrangulaires, d'un diamètre moyen de 2 millimètres, ont une cassure courte. Leur section transversale montre, à l'œil nu, une écorce très épaisse qui entoure un cylindre ligneux, étroit, présentant l'apparence d'une croix de Malte, à branches plus ou moins régulières (fig. 63).

\section{$2^{\circ}$ Caractères anatomiques $(\mathbf{1})$.}

Rhizome. - Le rhizome âgé présente, sur une coupe transversale, en allant de la périphérie vers le centre:

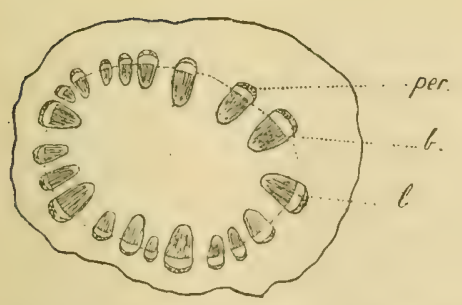

Fig. 60. Actra racemosa L. (Gross. 8/1). (Gross. 8/1). - Rhizome (port. jeune).

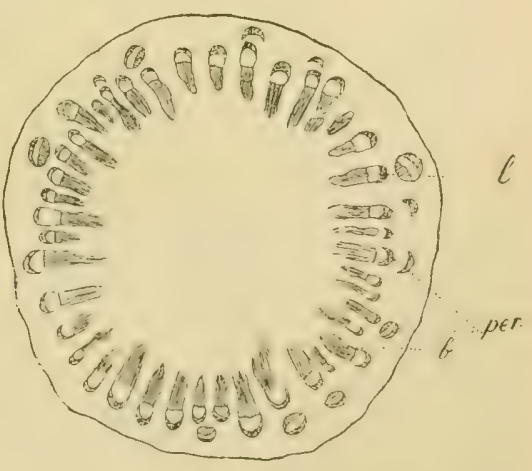

Fig. 61. Actæa racemosa L. (Gross. 8/1). - Rhizome (port. âgée).

(1) Malgré les notes et les figures relatives à la structure du rhizome de l'A. racemosa, donnés par Falck, Bastin, Planchor ex Colzix, bien des détails restent à donner sur cette structure. 

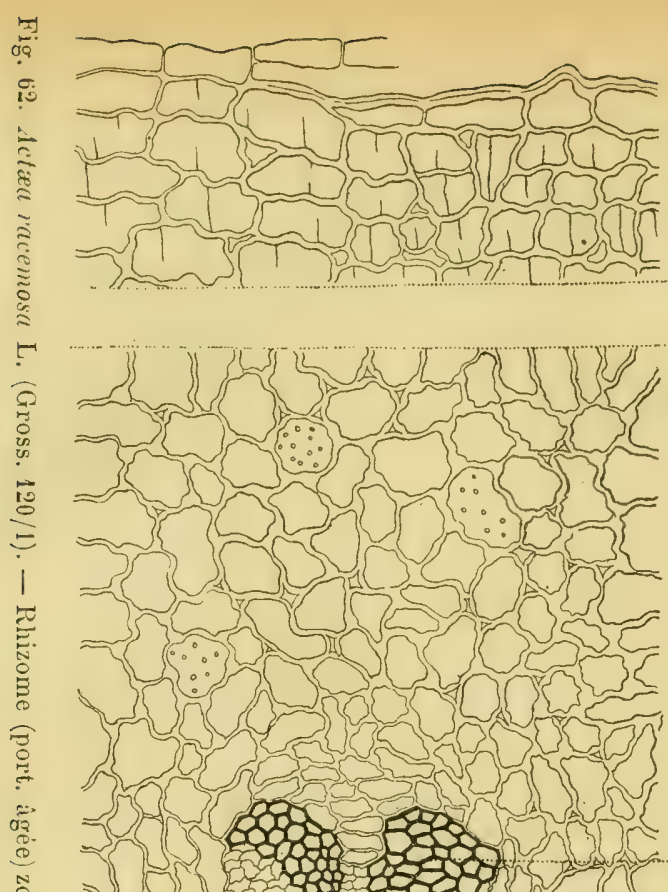

-2)

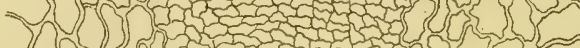
-C Onz

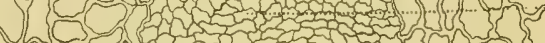

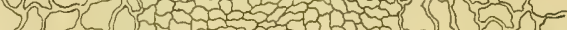

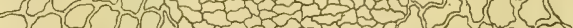

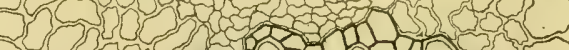
-1)

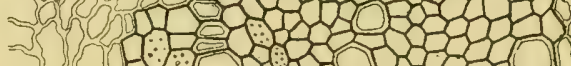

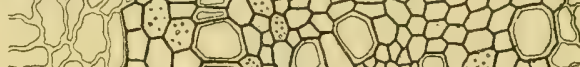

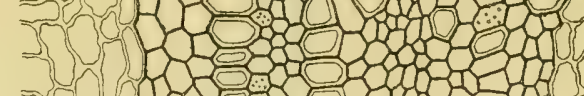
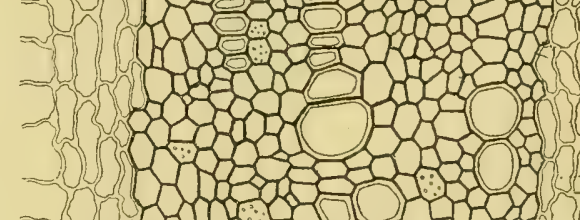

$2 . m$

per.

Z.

f.

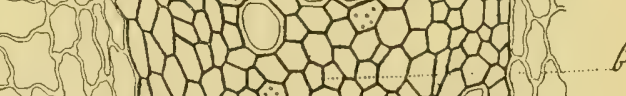


- Un parenchyme corlical, en partie exfolie is sa périphérie par la formation d'une assise protectrice d'aspect épidermoüde, formée de larges éléments, de taille quelque peu irrégulière, à paroi externe saillante, fortement épaissie, non lignifiée, à parois laterales et interne minces, quelque peu onduleuses.

La portion externe de l'écorce est formée d'éléments tabulaires, irrégulièrement stratifiés, à paroi onduleuse, médiocrement. épaisse, ponctuée, collenchymateuse, séparés par des méals irréguliers, et de taille variable.

La cavité de beaucoup de ces éléments est incomplètement divisée par de minces cloisons rompues, adhérant encore à l'une des faces de l'élément dont elles dépendent.

Au dos de chaque faisceau se trouve un arc péricyclique, miparti scléreux, mi-parti collenchymateux. Les éléments collenchymateux ont des angles fortement épaissis et sont séparés par de très petits méats.

Les éléments scléreux ont une paroi fort épaissie, une section elliptique.

Le liber, entièrement mou, est formé d'éléments aplatis, alignés en files radiales, à parois minces et onduleuses.

Le bois est formé de vaisseaux assez larges, à section circulaire ou elliptique, à parois relativement peu épaisses, lignifiées, alignés par petits groupes en files radiales; el d'un parenchyme, à éléments réguliers, polygonaux, alignés en files radiales, à paroi peu épaissie, fortement lignifiée, de diamètre plus considérable à la périphérie qu'à la pointe du faisceau.

La pointe de chaque faisceau ligneux est occupée par un parenchyme à éléments petits, irréguliers, à paroi mince, subcollenchymateuse, plus ou moins ondulée, au milieu desquels sont épars les plus petits vaisseaux.

Les rayons médullaires sont formés d'un parenchỵme, it éléments inégaux, séparés par de petits méats, la plupart allongés et alignés dans le sens radial, à parois minces, onduleuses, plus neltement collenchymateuses dans les assises qui limitent latéralement les faisceaux. 
Le parenchyme médullaire, homogène, présente tous les caractères du parenchyme cortical.

Racine. - Une radicelle, examinée au moment où s'établit la structure secondaire, présente, sur une coupe transversale:

Une assise périphérique dont les éléments, çà et là détachés, sont quelque peu irréguliers, à paroi externe fortement saillante, très épaissie, collenchymateuse, à paroi interne mince.

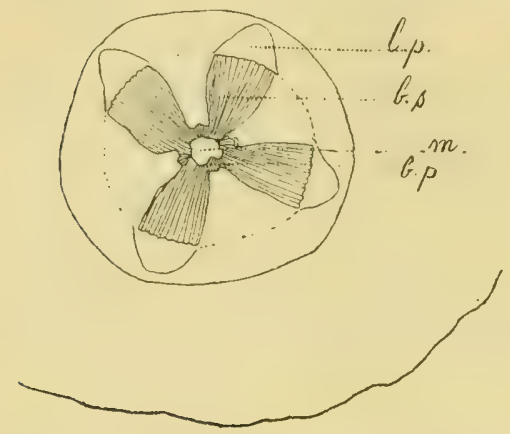

Fig. 63. Actra racemosa L. (Gr. 16/1). - Radicelle. Structure secondaire. (Semi-schém.). bp bois primaire, bs bois secondairc, $l p$ liber primaire, $m$ moelle.

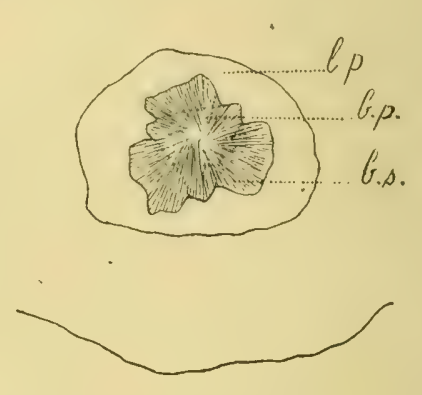

Fig. 64. Actæa racemosa (Gr. 16/1). Radicelle. Passage de la structure primaire à la structure secondaire. (Semi-schem.). bp bois primaire, $b s$, bois secondaire, lp liber primaire, $m$ moelle.

Le parenchyme cortical est entièrement formé de grands éléments, assez réguliers, à paroi très épaissie, collenchymateuse, avec de petits méats difficilement visibles.

L'endoderme, bien différencié, est formé d'éléments, plus ou moins aplatis et rectangulaires, à paroi mince, légèrement lignifiée, dépourvue de cadres d'épaississement.

Le péricycle est à peine distinct, par la taille un peu plus grande de ses éléments, du reste du conjonctif central, d'ailleurs réduit à des rayons nédullaires cunéiformes, car la moelle n'existe pas. Ces rayons sont formés d'éléments, diminuant de taille vers le centre, parenchymateux, à parois minces, disposés en files radiales. Les faisceaux libéro-ligneux (en train de se former, par constitution d'un hois secondaire en dedans des faisceaux libériens primaires), au nombre de 2, 3 ou 4, se réunissent en centre par leur pointe, et 
forment ainsi une figure assez analogue it une croix de Malte. Les faisceaux ligneux primilifs, à pointe externe, proéminent encore avec netteté dans la concavité des arcs limités par les faisceaux

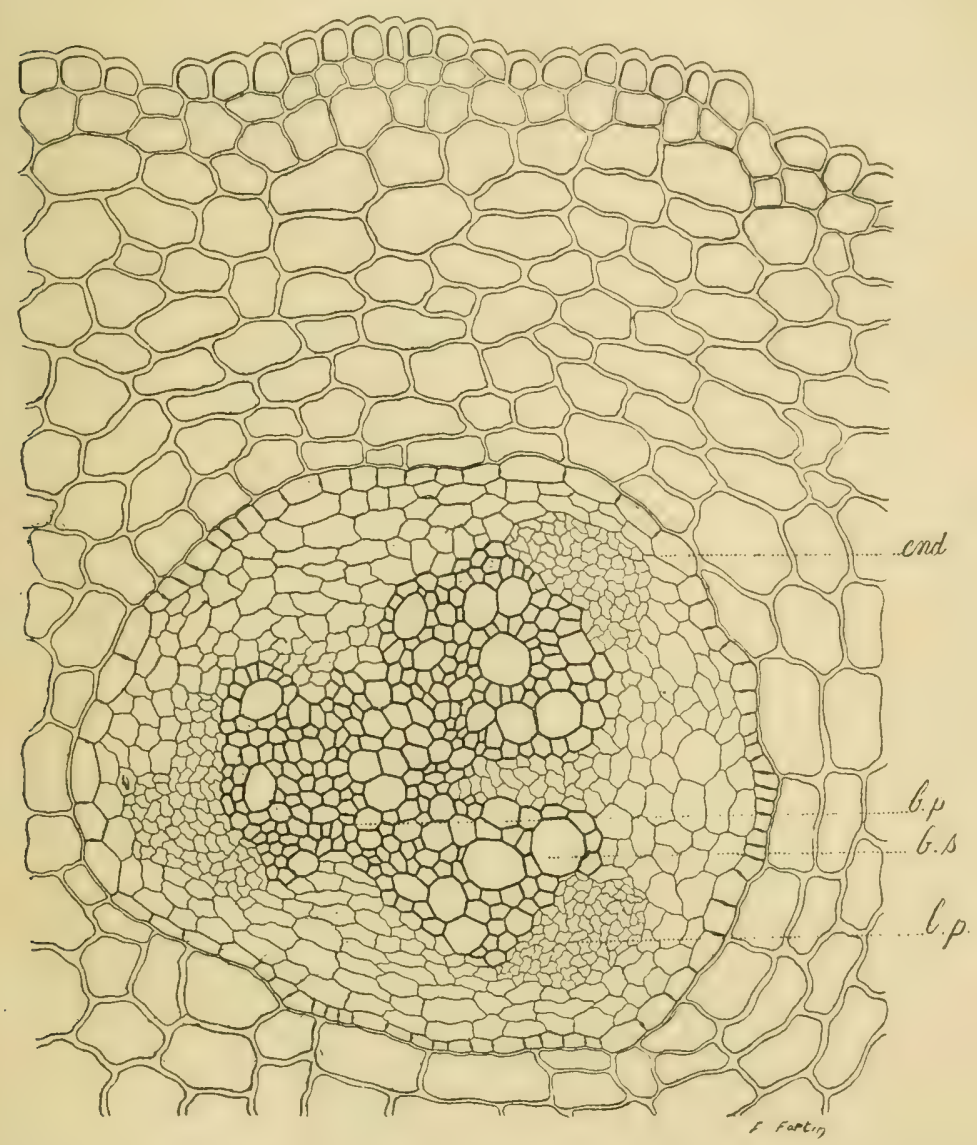

Fig, 65. Actra racemosa L. (Gross. 120/1). - Radicelle adventive. Passage à al a structure secondaire. $b p$ bois primaire, $b s$ bois secondaire, $l p$ liber primaire, end endoderme.

libéro-ligneux. Le bois de chaque faisceau est formé de vaisseaux et de parenchyme, offrant les caractères déjà décrits pour le bois du rhizome.

La racine plus àgée présente, avec une augmentation de diamètre de ses divers systèmes, essentiellement les mêmes caractères. 


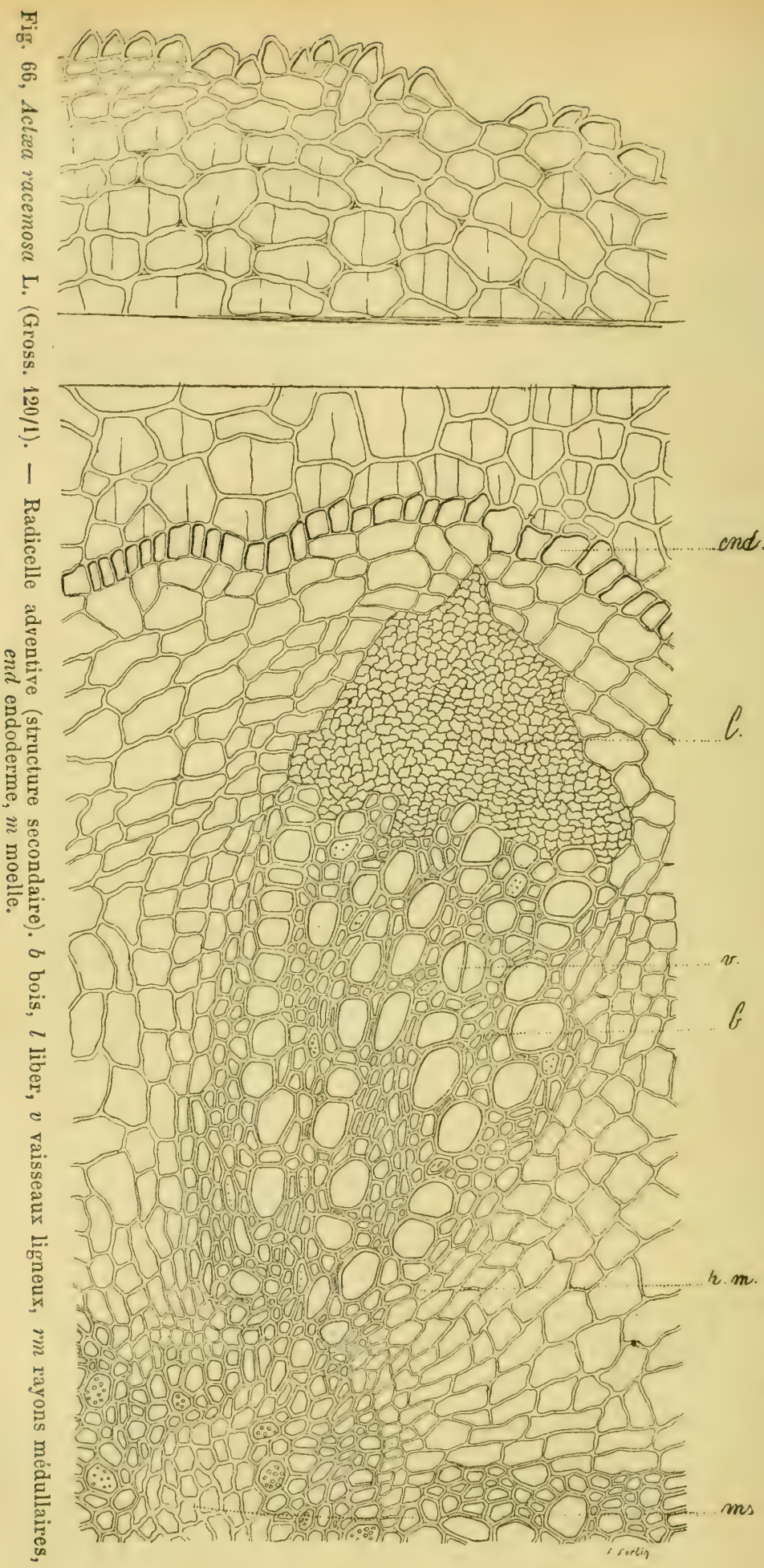


Les caractères diflérentiels (d'avec la racine jeune) les plus saillants sont l'apparition, datns la zone dr parenchyme yui entonte la pointe ligneuse de chaque faisereau, de fuclefurs ifements parenchymateux, polygonaux, a paroi lignilise, munie de larges pons:tuations, et la présence d'une moelle, d'ailleurs réeluile, oflranl les caractères des parenchymes corticaux et des layons midullaires.

Le parenchyme cortical du rhizome et de la racine pricuste une énorme quantité de grains d'amidon, dans presque toutes ses cellules. Ce sont des grains de laille très inegale, isolés ou le plus souvent groupés par 3, 4, et présentant alors une face externe convexe, et des faces latérales planes en contact; la plupart sont munis d'un hile punctiforme ou quelque peu linéaire, peu apparent.

BiBL. et-ICON.

1737. - LinNé (C.). Gen. (p. 644).

1793. - Linné (C.). Spec. (722).

1774. - Hornborg (J.). Planta cimicifuga $\left(8^{\circ} \mathrm{ou} 4^{\circ} \mathrm{Upsaliæ}\right.$ in Linn. Diss., VIII. 10 p. 1 tab. Amæn. acad., p. 169204).

1788-1807, - GenTNER. De fructibus (114).

1789. - Jussieu (A.-L.). Genera plant. (p. 235).

1790-1814. - Smith and Sowerby. Engl. Botany (13, 918).

1791-1801. - Dreves und Hayne. Bot. Bilderbuch (5, 134).

1798-1833. - Sтинм. Deutsch. Fl. (6, 21).

1802-38. - Svensk Botanik (291).

1805-46. - IIAYNE. Getreue Darstellung..... (1, 14).

1824. - De Candolle. Prodromus (I, p. 64).

1824. - ElliotT (ST.). Bot. of South Carolina (II, p. 16).

1828. - NeEs voN Esenbeck. Plant. medicin. (386, suppl, 17, $18,48)$.

1833-44. - Dietrich. Flora regni borussici $(6,407)$.

1833. - Hooker (IV. J.). Flora Boreali-Amerie. (I, p. 27).

1834-43. - BAXTER. Brit. Botany (3, 218).

1834-70. - Reighenibach (H. et G.). Icon. fl. German. (Ran. 4,121, f. 4759).

1836-50. - ExDlicher (St.). Genera plant. (n. 4799, n. 4800).

1839. - Spach (LD.). Suites à Buffon. (VII, p. 275).

1842. - WaLpeis (WV.). Repert. (I, p. 60).

1843. - TonRey: Flora of the State of New-York (4). 
1848-49. - Gray and Sprague. Genera Flor americ. bor. (20). 1848-68. - Walpers (W.). Annales bot. (IV, p. 32).

1848. - Grenier (C.) et Godron (D.). Flore de France (I, p. 51). 1850. - Pereira (J.). The elements of Mat.Med. (II, pt. 2, p.696). 1857. - PAyer (J.-B.). Traité d'organogénie comparée de la fleur (p. 253, pl. 58, fig. 36-43).

1861. - Bentley (R.). On Actæa, or Cimicifuga racemosa. (Pharmac. J., March.)

1861-70. - Willkomi (M.) et Lange (J.). Prodr. fl. Hisp. (3, p. 959).

1862. - Bentham et Hooker (Genera, I, p. q, n. 27).

1865. - Chapman (A.-W.). Flora of South. Unit. States (p. 11).

1866. - Baillon (II.). Adansonia (IV, p. 54).

1867. - GraY (A.). Manual of Botan. of Northern. U. States (ed. V, p. 48).

1867. - Baillon (H.). Dict. encycl. des se. méd. (I, 665).

1867. - - Hist. des pl. (I, p. 60,62, 88).

- Baillon (H.), Dupuis (A.) et Révoll (O.). Flore médic. $d u X I X$ e siècle (t. I, p. 19).

1871. - VAN DER Espt. De l'Actée à grappes (Cimicifuga racemosa). J. de méd., chirur. et pharmac. Bruxelles (III, 30-36).

1871. - Bentley. (Pharm. Journ. 2 (II, p. 460);

- (Am. Journ. of Pharm. (XXXIII, p. 396).

1871. - - ( Ibid. (XLIII, p. 151).

1872. - Year Book of Pharmacy (p. 385).

1874. - Flückigen (F.-A.) and Hanbuni (D.). Pharmeographia. A. History of the Principal Drugs of Vegetable Origin met with in Great Britain and British India, London (p. 16).

18\%. - Wood (G.-B.) and BACHE (F.). The Dispensatory of the United States of America (thirteenth edition. Philadelphia, p. 260).

1876. - Trson (E.). Actée in Baillon; Dict, de Bot. (p. 40-1).

1880. - Bentley (R.) and Trimen (H.). Medicinal Plants (I, pl. 8, London).

1884. - Marié (P.). Rech. sur la struct. des Renonculacées (Th. Fac. sc. Paris, sér. A, no 63, no d'ordre 523, p. 42, 46).

1884. - FALCK (M.-S.). (Am. Journ. of Pharmacy (56, p. 459).

1884. - - (Pharmac. Zeitung ( $n^{\circ} 87$, p. 749).

1890. - Garcin (A.-G.). Rech. sur l'histogenèse des péricarpes charnus (Th. Fac. sc. Paris, sér. A, $\mathrm{n}^{\circ} 144, \mathrm{n}^{\circ}$ d'ordre テ10, p. 226-28).

1890. - The Pharmacopoeia of the United States.

1893. - Roux (G.) et Foucaud (J.).Flore de France (t. I, p.54-5). 
1893. - Bastin (Ed. S.). (The Apothecary Chicago);

1894. - Dispensatory of the United States of America (sevent. ed.; p. $381382 \mathrm{fig}$.).

1895. - BASTIN (Ed.S.). Structure of Cimicifuga (Americ: Journ, of Pharm. vol. 67, no 3, march, p. 121-8, fig. 1, 2, 3, $4,5,6,7)$.

1895. - I'laichon (G.) et Collin (E.). Les drogues simples d'origine végetale (II, p. 930-32, fig. 1377, 78, 79).

1896. - Robinson (L.). Synoptical Flora of North America (first fasc. Ranunculacex). 


\section{CHAPITRE II}

\section{E TUDE GHIMIQUE}

\section{A. - Actæa spicata.}

Aucune analyse sérieuse des divers organes d'Actie spicata n'a été faite jusqu'ici. Les quelques données éparses sur ce sujet sont tellement vagues, qu'elles ne valent point la peine d'être relatées. Nous avons soumis à l'analyse immédiate, suivant la méthode exposée précédemment (v. p. 61), les divers organes de cette plante. Voici les résultats de ces analyses :

ANALYSE IMMÉdiATE DES ORGANES D'AGTEA SPIGATA

Rhizome (sur 10 gr. réduits en poudre)

1. Alcool bouillant. Graisse (très peu).

Acide gallique (traces).

2. Ėther bouillant. Traces d'alcaloïdes.

Matières minérales.

Résine.

Tannin.

Glucose.

Glucosides en petite quantité.

3. Eau

Matières minérales.

Tannins.

Albuminoïdes.

Sucres réducteurs.

4. $\mathrm{NaOH}$ à $3 \%$. Albuminoïdes.

Tannins.

ร. $\mathrm{HCl}$ à $1 \%$. Matières minérales.

Sucres dédoublés en assez grande quantité.

6. Résidu. Cellulose.

Ligneux. 
Feuilles (sur 3 gre réduits en poudre):

1. Ether.

\section{Ether}

Pas d'alcaloïdes.

Graisse.

Acide gallique (traces).

Chlorophylle.

Résine.

\section{Alcool.}

Pas d'alcaloïdes.

Matières minérales.

Tannins.

Sucres réduits.

Glucosides.

Résine.

3. Eau.

Matières minérales.

Tannins.

Albuminoïdes.

Pas de sucres réduits. non réduits.

4. NaOH à $3 \%$. Tannins (traces).

Albuminoïdes (traces).

รั. $\mathrm{HCl}$ à $1 \%$.

Matières minérales.

Sucres réduits venant des hydrates de carbone dédoublés.
6. Résidu.
Cellulose.
Ligneux.

Péricarpe isolẻ. - Les fruits ont été conservés dans l'alcool : à cause de cette macération, la proportion des principes actifs doit être plus faible.
1. Ether.
Pas d'alcaloïdes.
Graisses (traces).
Acide gallique (traces).
Résine (peu). 
2. Alcool. Pas d'alcaloïdes.

Matières minérales.

- colorantes.

Tannins.

Sucres réduits (très peu).

3. Ean. Sels minéraux,

Albuminoïdes.

Tannins.

Sucres réduits (traces).

- non réduits (tracesj.

4. NaOHo $3 \%$. Matières pectiques.

Albuminoïdes.

Tannins.

э. $\mathrm{HCl} 1 \%$ Matières minérales.

Hydrates de C dédoublés (peu).

6. Résidu. Cellulose.

Ligneux.

Graines isolées (fraìches).

1. Ether. Chlorophylles (?) (traces).

Graisses (en assez grande quantité).

2. Alcool. Alcaloïdes (traces) (des plus douteux).

Tannins.

Glucose (en assez grande quantité).

Glucosides (peu).

Sels minéraux.

3. Eau.

Tannins (peu).

Albuminoïdes.

Matières minérales.

4. NaOH $3 \%$. Tannins.

Albuminoïdes.

5. HCl 1\% \% Hydrates de carbone dédoublés.

Matières minérales. 
Guidé par ces premiers essais, nous avons recherché, sur une guantité plus considérable ( 700 grammes de fruits complets (péricarpe et graine), les corps actils susceptibles de se trouver dans ces organes.

Ces fruits, conservés dans l'alcool, sont débarrassés de celui-ci par distillation. On prépare avec le résidu un extrait aqueux, où les alcaloïdes sont recherchés par deux méthodes :

fo Par traitement de l'extrait aqueux par la potasse et agitation à l'éther;

2 Par précipitation de l'extrait aqueux par le sous-acétate de plomb et filtration. Le liquide filtré a été débarrassé du plomb par un courant d'hydrogène sulfuré: puis il a été filtré et concentré dans le vide; on l'a ensuite agité avec de l'éther, il a donné par évaporation un résidu très faible, dont l'essai physiologique montre l'innocuité (V.p. 239). On a fait alors l'extrait alcoolique des fruits; on y a recherché les résines en chassant l'alcool, reprenant par la potasse étendue, décolorant par le noir animal et concentrant le liquide filtré dans le vide.

Le liquide ainsi obtenu, assez riche en produits résineux, est réservé pour un essai physiologique (V. p. 239).

En vue de l'extraction d'une certaine quantité de résine, 4 kilos de feuilles sèches d'Actra spicata sont soumis au traitement suivant: les feuilles, réduites en poudre, sont épuisées par 30 litres dalcool fort; le liquide alcoolique résultant de ce traitement est évaporé à sec dans le vide au bain-marie. Le résidu est épuisé par l'eau, qui enlève certaines matières (tanins et glucosides) qui souillent la résine restante.

Après cet éptisement à l'eau, le corps résinoïde [ident. avec l'actéine d'Actę racemosı, ì établir ultérieurement ] est purifié par redissolution dans l'alcool fort et nouvelle précipitation par l'eau : on le sèche ensuite dans le vide.

Ce traitement ne donne qu'un résultat médiocre : beaucoup de chlorophylle, beaucoup de matières grasses, et environ 2 grammes de résine par kilo.

Le corps résinoïle extrait à l'état impur du rhizome d'A. spicata semble bien identique à celui extrait d'A. racemosa, dont nous 
indiquons plus loin les propriélés. Nous n'arons cependant pas eu a notre disposition une quạntité suffisante de ce produit pour nous prononcer en faveur d'une identiticalion complète. Ce point spécial réclame de nouvelles recherches.

Ces feuilles contenant aussi un glucoside, on a cherché à l'extraire du produit de lavage par l'eau du résidu ci-dessus, résultant de l'épuisement des feuilles par l'alcool.

La combinaison, possible à prévoir, des tanins avec les glucosides est détruite par l'acétate de plomb ou la chaux. Le résultat de cet essai est absolument nul, tant est faible la proportion de glucosides contenue dans les feuilles.

En manipulant les divers organes d'A. spicata, on ne peut manquer d'être frappé de ce fait, que leur macération clans l'eau mousse avec une extrême facilité. L'extrait alcoolique des diverses parties de la plante mousse encore mieux. On est done en droit de songer, à priori, à la présence d'une saponine. Cette. hypothèse n'est point justifiée. Le glucoside que la plante renferme en si faible quantité n'est pas identique à la saponine; c'est la présence de la résine, surtout abondante dans les solutions alcooliques, qui leur communique la propriété de faire mousser l'eau.

On sait que, dans un très grand nombre de Renonculacées et, en particulier, chez A. racemosa, on a signalé la présence de principes volatils, facilement destructibles, et auxquels ces plantes fraiches devraient la majeure partie de leurs propriétés toxiques. Chez A. spirata, aucun principe semblable ne nous est décelé par l'analyse chimique, alors même que celle-ci est pratiquée sur la plante vivante. 


\section{B. - Actæa racemosa.}

\section{RECHERCHES ANTERRIEURES}

La plus extrême confusion règne daus les opinions des auteurs, qui se sont occupés des principes actifs d'A. raremeste Silns pitrler de la gomme, des matières grasses, de l'amidon, du sucre et de divers sels minéraux signalés par tous les auteurs, on a indiqué dans le rhizome de cette plante: deux matières colorantes, l'une brune, l'autre verte, résine, tanin, acide gallique (Trlgnmax). Fraiche, la plante contiendrait une huile volatile guon pent obtenir par distillation, dont l'odeur spéciale rapletle celle du rhizome, un sucre incristallisable et deux résines distincles: l'une soluble dans l'alcool, insoluble dans l'éther, l'autre soluble dans ces deux liquides (Davis). Coxard conclut à l'absence, même dans la plante fraiche, d'une substance volatile fournie par lat distillation; mais it y a trouvé une substance cristallisable neutre, très àcre, d'un beau jaune, insoluble dans l'eau, soluble dans l'ether, legèrement soluble dans l'alcool et le chloroforme. Cet auteur est d'ailleurs muet sur la composition et les propriétés physiologiques de cette substance.

BEACI a obtenu le même principe (cimicifugine ou macrotine) par la méthode de Conand.

Trimble n'aurait isolé qu'un principe amorphe.

Falak dit aroir isolé un alcaloïde (identique au corps ṕrécédent?) soluble dans l'alcool concentré, dont les solutions précipitent par l'acide phospho-molybdique, le timin et liodure double de Ke IIn. Ce corps, soumis à la fusion en présence de potasse, émettrait des rapeurs colorant en bleu le papier de tournesol; il émettrait des fumées blanches, épaisses, à l'approche d'une baguette trempée dans $\mathrm{HCl}$; se dissoudrait dans $\mathrm{SO}^{4} \mathrm{H}^{2}$ concentré en donnant une solution brune, passant au jaune par l'addition de bichromate de potasse.

GaLlanen croit, au contraire, que le principe eristallisable isolé par ses prédécesseurs et indiqué par eux, comme le principe actif, ne serait que du sucre de canne, qu'il a pu extraire de l'extrait fluide.

Contrairement aux résultats amnoncés par Gurdki, BEACu, Llutd 
et Wormsx n'ont obtenu, at l'analyse du rhizome d'A.rucemosa, qu'un produit impur (cimicifugine), formé par le mélange d’une résine difficilement soluble dans l'alcool et dans l'éther, et d'un résidu insoluble dans ces réactifs.

\section{RECHERCHES PERSONNELLES.}

Analyse immédiate du rhizome d'Actæa racemosa (sur 10 grammes réduits en poudre).

1. Ether bouillant. Pas d'huiles essentielles.

Résidu sol. dans alcool, résine.

Pas de graisse (ou traces).

Pas d'acide gallique.

2. Alcool bouillant. Sels minéraux.

Résine précipité par eau.

Tanins (col. brune par $\mathrm{FeCl}^{\circ}$ ).

Glucoses (réduit liq. de Fehling).

Glucosides (réd. liq. de Fehling, plus fortement après qu'avant interversion).

Comme produits principaux il semble, outre la résine, y avoir un glucoside - (qui souille peut-être la résine, ce qui a fait croire au pouvoir réducteur de cette dernière?).

3. Eau bouillante. Sels minéraux.

Albuminoïdes.

Tanins forte (col. brune par $\left.\mathrm{Fe}^{2} \mathrm{Cl}^{5}\right)$.

Sucres (très peu; presque pas de réduction de la liqueur de Fehling).

4. NaoH à $3 \%$. Albuminoïdes.

Tanins (col, brune par $\left.\mathrm{Fe}^{2} \mathrm{Cl}^{5}\right)$.

๖. HCl à $1 \%$. Hydrates de C dédoublés (réd. liq. de Fehling).

6. Résidu. Ligneux.

Cellulose.

Celle analyse immédiate nous permet déjà de conclure à l'absence d'alcaloüde. Pour plus de sûreté, nous arons procédé à la 
recherche des principes alcaloïdirgues sur une forte quantilé de rhizome.

Après macération prolongée, on l'épuise par l'alcool dans un appareil à déplacement, on sature les acides et précipite les tanins par addition d'un peu de lait de chaux, puis de sous-acélate de plomb étendu d'alcool, on filtre en vase clos pour séparer le dépôt; ce dernier est lavé, toujours avec le mème alcool. Dans la

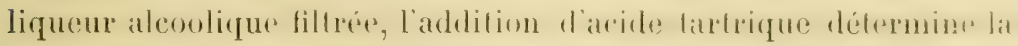
précipitation de l'excès de plomb et de chaux resté en solution.

Après nouvelle filtration, le liquide est distillé; le résidu de la distillalion est forme par une masto prinumse plus ou moins pu suspension dans une liqueur acide; celle-ei filtrée et alcalinisée est agitée arec de grandes quantités d'éther, puis de chloroforme. Ces dissolvants, après distillation, laissent de faibles résidus dans lesquels il est impossible de constater la présence d'un alcaloïde. La liqqueur acide primitive, de même que ces derniers résidus, ne donnent aucun précipité par les réactifs de Walser et de Bouchardat.

\section{Extraction du principe actif.}

Voici le procédé qui permet, en opérant sur une forte quantité de rhizome (10 kilos), d'obtenir le meilleur rendement pour le corps que l'analyse physiologique nous montrera pourvu d'activite.

Le rhizome, réduit en poudre grossière, est mis à macérer pendant deux jours, dans deux fois son poids d'alcool à $80^{\circ}$, puis introduit dans un appareil à déplacement et lessivé avec de l'alcool au mème degré, jusqu'à obtention de 6 litres par kilo de poudre employée. Dans ce liquide, on ajoute un peu de lait de chaux qui sature les acides et commence la précipitation des tanins; cette précipitation s'achève par l'addition de sous-acétate de plomb, étendu d'alcool. Quand il ne se produit plus de précipité, on filtre en vase clos pour séparer ce dépót; ce dernier est lavé toujours avec le mème alcool,

La liqueur ainsi obtenue est ambrée, on en achève la décoloration en la filtrant dans une allonge, sur une colonne de noir animal granulé (il faut pour obtenir une liqueur presque incolore $500 \mathrm{gr}$. de noir par kilo de rhizome). L'alcool est ensuite séparé par distillation, à la température la plus basse possible.

Le résidu de la distillation est un corps résinol̈e, visqueux, nageant 
dans un peu d'eau; il est lavé à plusieurs reprises dans de l'eau distillée chaude, puis redissous dans environ deux fois son poids d'alcool.

Lorsque la dissolution est bien complète, on ajoute goutte à goutte une solution alcoolique d'acide tartrique, afin de précipiter les traces de chaux et de plomb qui n'auraient pas été entrainées par les lavages. Lorsque l'acide tartrique ne produit plus de trouble, on filtre et on verse le tout dans environ quatre à cinq fois son volume d'eau distillée, froide. Ise produit résinoïde qui se sépare est lavé, malaxé, puis séché à l'air en couches très minces, la température de l'étuve suffit pour le contracter et le colorer légèrement.

Par ce procédé, on retire de $3 \check{a}$ à $40 \mathrm{gr}$. dudit corps, par kilo de rhizome. C'est ce corps que nous désignerons, pour les raisons indiquées plus loin. sous le nom de corps $A$ ou Actéine.

Indiquons ici, brièvement, une des premières méthodes employées au début de ces recherches pour isoler ce corps. Après défécation, la liqueur mère alcoolique est filtrée, puis additionnée d'acide tartrique jusqu'à cessation de tout précipité. Après nouvelle filtration, le liquide est distillé. Le résidu résinoïde est traité par le chloroforme (il ne s'y dissout qu'en partie), décoloré par le noir animal. La solution chloroformique distillée donne un résidu, qui est traité par l'éther de pétrole, puis par la benzine, puis enfin par l'éther ordinaire. Le premier de ces dissolvants n'a rien dissous, le second des traces très faibles, l'éther en dissout, ainsi que l'alcool, une forte proportion.

En abandonnant à l'évaporation spontanée une quelconque de ces solutions, on obtient toujours des parcelles d'actéine.

L'actéine semble bien être voisine du corps isolé par Conard.

La marche suivie par cet auteur pour isoler le principe qu'il déclare cristallisable, est la suivante :

La teinture du rhizome est traitée par une solution de sous-acétate de plomb qui précipite la résine, le tanin, les matières colorantes; on filtre, et l'excès de plomb est précipité par l'hydrogène sulfuré. On filtre à nouveau et la liqueur est abandonnée à l'évaporation spontanée. On 
oltient ainsi un résidu pulvérulent, qui est traité par la benzine, lavé à l'eau, dissous dans un excès d'alcool concentré. Isil solution alcoolique est abandonnée à l'évaporation, jusqu'ì obtention d'un résidu sec, qui est repris par l'alcool, puis soumis à nouveau i l'évaporation. On obtient finalement une masse cristalline, dont l'apparence extérieure n'est pas sans analogie avec l'alun.

Cette substance est peu sapide, mais âcre, insoluble dans la salive, mais soluble dans l'alcool, surtout à chaud, dans le chloroforme, légèrement soluble dans l'éther, fusible et inflammable, à réaction neutre.

Le corps isolé par Coxıro a été désigné sous le nom de Cimicifugine. Mais d'une manière courante, en Amérique, on désigne sous ce nom le produit résineux impur, obtenu par précipation de la teinture alcoolique (v. p. 229). Pour ériter toute confusion, nous proposons, au moins provisoirment, le nom d'Acténe, pour désigner le corps ảéfini que nous avons obtenu.

Il est possible de retirer aussi, des eaux mères de préparation du corps précédent, un autre corps que, pour ne rien préjuger de sa nature, nous désignerons sous le nom de corps $B$. Ce corps peut être extrait très pur, et à l'état cristallisé, par une des manipulations suivantes :

$1^{\circ}$ La liqueur alcoolique ambrée (obtenue comme il est indiqué cidessus) qui contient en dissolution l'actéine (et qui a subi déjà l'action du lait de chaux et du sous-acétate de plomb), est filtre sur du noir animal. La première portion qui filtre est complètement incolore; si on l'abandonne à l'éraporation spontanée dans une capsule de verre, on obtient une couche vitreuse, qui ne tarde pas i perdre sa transparence par suite de la production de petils cristaux.

$2^{\circ} \mathrm{La}$ liqueur alcoolique ci-dessus, après filtration lente sur le noir, donne, après distillation, une résine visqueuse, qui est lavée à l'eau distillée chaude, puis redissoute dans deux fois son poids d'alcool. On l'additionne alors à nouveau d'un peu de sous-acétate de plomb, on filtre pour séparer le dépòt, et on ajoute seulement alors la solution alcoolique d'acide tartrique; on filtre de nouveau; le ballon dans lequel on a reçu la liqueur est, après quelques jours, tapissé de petits cristaux.

$3^{\circ}$ Si la solution d'actéine dans l'eau alcoolique ( $3^{\mathrm{e}}$ stade de sa préparation, avant le lavage et le séchage définitifs) est soumise à l'évaporation à $60^{\circ}-70^{\circ}$ pour en chasser l'alcool, on voit se former un dépôt 
résinoïde compact. Ce dépôt traité par l'acide tartrique, puis filtré, donne, dans le ballon où on reçoit le produit de la filtration, des cristaux, en quantité un peu plus abondante que par les procédés ci-dessus, mais toujours en très faible proportion.

Toutes les parcelles obtenues par les manipulations ci-dessus ne s'élèvent pas à plus de 4 gr. 50 pour 10 kilos de rhizome.

Commme nous le dirons plus loin, il semble bien que ce corps B ne doit etre qu'un produit de l’hydrolyse partielle de l'actéine, sous l'influence de la chaux el du sel de plomb employés pour la préparation de celle-ci. Il y aura donc lieu d'essayer une autre procédé d'extraction, dont seront bannis tous les agents plus ou moins capables d'hydrolyser l'actéine. Nos essais, dans cette voie, sont trop peu avancés pour pouvoir être utilement rapportés ici.

L'actéine, telle que nous l'avons obtenue, peut être mêlée à une quantité très faible du corps B. Mais cette quantité est si faible qu'elle semble devoir ètre pratiquement considérée comme nulle.

En effet l'actéine est très soluble dans l'acide acétique, tandis que le corps B ne l'est pas. Or la dissolution de l'actéine telle que nous l'avons obtenue) dans l'acicle acétique ne laisse aucun résidu insoluble; régénérée de sa solution acétique, elleprésente le mème point de fusion et les mêmes propriétés que le corps primitif.

L'actéine sembie bien ìtre un corps homogèneet non un mélange. Vient-on, en effet, à la fractionner par solution dans l'alcool, puis par la précipitation partielle par évaporation de l'alcool ou aduition d'eau, on a deux portions, fondant toutes deux à $170^{\circ}$.

La différence des points de fusion du produit ainsi obtenu et de l'actéine primitive, tient peut-être à ce que l'actéine subit, par l'ébullition en solution alcoolique, un commencement d'altération.

La très faible proportion du corps B, obtenue par les traitements indiqués, s'explique peut-ètre par le mode même de préparation. Lors du défécage par la chaux et le plomb de la solution mère alcoo- 
lique, une notable quantité de ce corps se forme, peut-être aux dépens de l'actéine. Mais, comme le corps Best è prine soluble dins l'alcool, il doit ètre entrainé aree les précipilis calciques of plombiques, el seule, la petite quantité qu'est susceptible de dissoudre la solution mère, se retrouverail à la fin du traitement.

L'actéine, après lavage ì fond par l'eau, présente le même point de fusion que le principe primitif.

L'essai ci-dessous le prouve :

De l'actéine, fondant à $130-153^{\circ}$, est lavée à fond; le corps'purifié par ce lavagé, fond instantanément à $225-230^{\circ}$, puis progressivement à partir de $175^{\circ}$. Voici la raison de cette anomalie : le corps primitif et le corps obtenu par lavage sont identirues, mais les particules du second sont agglomérées en petits fragments, cette simple cause mécanique détermine l'augmentation du point de fusion. Ce qui prouve le bien-fondé de cette explication, c'est que les 2 corps, réduits en poudre, fondent tous deux instantanément à $153^{\circ}$.

Done, notre actéine n'est pas un mélange, dont un des composants (glucoside par exemple), serait soluble dans l'eau. C'est un corps homogène, à point de fusion bien défini.

\section{Recherche du saccharose.}

Pour nous rendre compte des raisons qui araient pu porter G.sLLAIter ì déclarer que le seul principe cristallisable de l'A trtere raremosa était du saccharose, nous avons procédé à la recherche du sucre de canne dans le rhizome.

La plante sèche est réduite en poudre, épuisée par l'eau chaude, le liquide filtré est déféqué par le sous-acétate de plomb, l'excès de plomb précipité par $\mathrm{H}^{2} \mathrm{~S}$, le liquide filtré concentré dans le vide au bain-marie. Il est impossible d'obtenir aucun cristal de saccharose. Le sirop réduit directement la liqueur de Fehling.

Rem. - On pourrait à la rigueur objecter que le traitement du sirop déféqué par $I^{2} \mathrm{~S}$ met en liberté une faible quantité d'acide acétique, qui pourrait, à priori, opérer une légère interversion du sucre de canne pendant la concentration. Mais cette objection ne laisserait pas que d'être assez spécieuse.

La recherche du saccharose est donc négative; on ne peut extraire du rhizome qu'un sucre directement réductible. 


\section{Propriẻtés de l'actéine}

C'est un corps d'un goûtâcre, insoluble dans l'eau même chaude, mais lui communiquant la propriéte de mousser; ì peine soluble dans l'ether, soluble dans la benzine et le chloroforme, peu soluble dans les alcalis, indéfiniment soluble dans l'alcool fort, à froid. L'alcool à $2 \partial \%$ ne dissout que $0 \mathrm{gr}$. 001 de ce corps pur par centimètre cube. L'ammoniaque en dissout difficilement une faible quantité, la lessive de potasse ou de soude le dissolvent plus facilement (surtout en étendant d'un peu d'eau); l'addition d'un acide à la solution alcaline en amène la précipitation. Très soluble dans l'acide acétique froid, sans coloration, insoluble dans l'acide azotique à froid, soluble avec coloration jaune dans l'acide chlorhydrique, et se détruisant dans le même acide à chaud. Ce corps devient mou dans les solutions chaudes, il se comporte comme un corps neutre.

Trailée par le bichromate de potasse, puis par une goutte de $\mathrm{S}^{4} \mathrm{H}^{2}$, l'actéine donne une coloration d'un violet sale, passant rapidement au vert.

$\mathrm{SO}^{\prime} \mathrm{H}^{2}$ concentré le dissout en partie, et lui imprime une coloration d'abord rouge brune, puis noire. La solution alcoolique reste incolore et ne précipite pas en présence de chloral (même à chaud), de $\mathrm{KI}$ iodé, de perchlorure de fer, l'addition d'accilate neutre de $\mathrm{Pb} y$ détermine un précipitéblane soluble dans l'alcool,celle d'eau de $\mathrm{Br}$ un pricipité blanc abondant, l'addition d'ammoniaque n'y détermine pas trace de précipité.

La solution alcoolique dégage une odeur forte, assez agréable, et qui rappelle quelque peu celle de la fleur d'oranger.

Récupéré de sa solution acétique, le corps se présente sous le même état et avec les mêmes propriétés.

Examinće au microscope, l'actéine ne présente pas de véritables formes cristallines, on ne voit que des lamelles, dont les plus 
grosses présentent des cassures, qui rappellent cellesdes substances résineuses.

\section{Essais d'hydrolyse de l'actéine}

$1^{\circ}$ En suspension dans l'eau. - 2 gr. 5 d'actéine sont chauffés, pendant 3 heures a $120^{\circ}$, avec ${ }^{\circ} 0$ grammesd'eatu ot 2 gr. On sature par $\mathrm{CO}^{3} \mathrm{Ba}$, on filtre et on concentre dans le vide; cette solution réduit difficilement la liqueur de F́hling, et a un pouroir rotatoire presque nul. Deux autres essatis d’unterversion parle mème procédé, en opérant avec 5 grammes de résine, donnent le même résultat. L'interversion est plus facile en chauffant à l'autoclave, à $120^{\circ}$ pendant 3 heures, avec une solution de $\mathrm{SO}^{\prime} \mathrm{H}^{2}$ à $ّ \%$. La solution neutralisée par $\mathrm{CO}^{3} \mathrm{Ba}$, réduit le nitrate d'argent.

La solution obtenue avec à grammes d'actéine soumise à l'autoclave à l'interversion incomplète par $\mathrm{SO}^{4} \mathrm{H}^{2}$, est mise à chauffer de nouveau dans un tube scellé, pendant 3 heures à $200^{\circ}$, arec une solution de $\mathrm{SO}^{4} \mathrm{H}^{2}$ à $10 \%$. L'interversion est encore très faible.

$2^{\circ}$ En solution alcoolique. - La solution alcoolique d'actéine est additionnée d'HCl à $6 \%$ jusqu'à apparition d'une teinte lactescente (afin de bien diviser le corps), on maintient le tout à la température de $80^{\circ}$ environ, pendant 2 heures̉; on chasse l'alcool et on filtre; le liquide filtré réduit la liqueur de Fehling.

Deux grammes d'actéine sont soumis à l'hydrolyse comme cidessus; on filtre, la liqueur filtrée réduit la liqueur de Fehling; le résidu de l'hydrolyse fond à 120-1250, le corps réduit formé correspond à $13 \%$ du poids de l'actéine primitive; sa solution prend par le traitement avec l'orcine et $\mathrm{HCl}$ une coloration rouge (caractère des sucres en $\mathrm{C}^{6}$ ) et présente un pouvoir rotatoire dextrogyre.

La solution d'actéine dans $\mathrm{S}^{4} \mathrm{H}^{2}$ concentré donne, lorsqu'on l'étend d'eau, unc solution trouble rose, qui, après filtration, donne un liquide rose, réduisant la liqueur de Fehling.

La difficulté extrême avec laquelle l'actéine s'hydrolỵse, donnerait à douter qu'elle apparlienne au groupe des silucosides. Ce n'est que provisoirement que nous la considérons comme telle. 


\section{Propriétes du corps B}

Le corps B, cristallisé, est presque insoluble dans l'eau, même à chaud, bien moins soluble que l'actéine, dans l'alcool, presque insoluble daus les alcalis lioll, Na()H, Azll'OH), a peu près insoIuble dans l'éther, un peu soluble dans la benzine et le chloroforme, plus lentement et moins soluble dans l'acide acétique que l'actéine. So ${ }^{4} 1^{2}$ l'attaque en produisant une coloration brune; $\mathrm{AzO}^{5}$ et $\mathrm{HGl}$ ne produisent rien, et à chaud l'attaquent, KI iodé y détermine un précipité jaunâtre, si peu notable qu'il est à peine visible.

L'addition à la solution alcoolique de perchlorure de fer, d'ammoniaque, de sous-acétate de plomb, d'eau de brome n'y détermine rien (comme forcément, par celte addition, la solution alcoolique se trouve étendue d'eau, il y a formation d'un louche, par légère précipitation du corps insoluble dans l'eau. Le point de fusion est 24̋̈-230 . Son interversion est très difficile mèmes assais d'hydrolyse que ci-dessus), la liqueur résultant de cette opération prend une coloration rouge avec l'orcine chlorhydrique; une portion de ce corps interverti est séchée, puis traitée par l'acétate de phénylhydrazine, aucun résultat net; on essaie à nouveau l'interversion en tube scellé à $200^{\circ}$, pendant 3 heures avec $\mathrm{SO}^{\prime} \mathrm{H}^{2}$ à $10 \%$, la solution réduit à peine la liqueur de Fehling.

On est en droit de supposer qüil existe dans la plante un ferment soluble, capable de dédoubler plus facilement le glueoside qui s'y rencontre, que les agents habituels d'hydrolyse du laboratoire. Aussi avons nous procédé à la :

Recherche d'une diastase dans le rhizome frais (à la période du repos) d'Actra spicata. On fait digérer le rhizome frais dans de l'eau distillée à $33^{\circ}-40^{\circ}$ pendant plusieurs heures; on divise la macération en deux portions, l'une est précipitée paī l'alcool (albuminoïdes), l'aulre est additionne de phosphate d'ammoniaque et précipitée par le chlorure de calcium. Les deux précipités sont filtrés et séchés dans le vide. 
On dispose les expériences suivantes :

$1^{\circ}$ Dans un tube à essai, à l'étuve à $3 \mathrm{~J}^{\circ}$, on met "ٌ̈ $\mathrm{c}^{3}$. d'eau, 0 gr. 10 d'actéine et un peu du précipité alcoolique ci-dessus.

$2^{\circ}$ Même expérience, après ébullition destinée à détruire le ferment soluble supposé.

$3^{\circ}$ Mème expérience que la $1^{\text {re }}$, mais en se servant du précipité par le phosphate de chaux.

$4^{0}$ Même expérience que la $3^{\mathrm{c}}$, après ébullition.

Tous ces essais donnent un résultat négatif. Il n'y a pas trace d'hydrolyse de l'actéine épreuve de réduction par la liqueur de Fehling,.

Il eût été évidemment plus probant de s'adresser à l'A. racemosa d'où est extrate l'actéine. Mais nous n'avions pas à notre disposition d'échantillon authentique vivant de cette espèce.

\section{Analyse élémentaire de l'actéine et du corps B.}

Nous croyons devoir rapporter ici les résultats d'une analyse élémentaire (préliminaire) de l'actéine el du corps B qui en dérive. Mais il importe de remarquer que les dosages n'ayant été effectués qu'une seule fois, il peut y avoir quelques réserves à faire, au sujet de la valeur absolue des chiffres obtenus.

Actéine.

$$
\begin{aligned}
& \text { Mat. S. } 2.7510 \\
& \frac{2.2290}{0.5220} \\
& \mathrm{H}^{2} \mathrm{O} 45-\quad 3.3020 \\
& \frac{4.9040}{0.3980} \\
& \mathrm{Co}^{2} 90-\quad 4.3990 \\
& 3.1770 \\
& \frac{1.2220}{\mathrm{H}}=8.47 \% \\
& \mathrm{C}=63.83 \\
& \mathrm{O}=27.70
\end{aligned}
$$

Correspondant à

$$
\text { ou } \begin{array}{lll}
\mathrm{H}^{8}, 470 & \mathrm{C}^{83}, 319 & \mathrm{O}^{1}, 731 \\
\mathrm{C}^{53} & \mathrm{O}^{17}
\end{array}
$$

\section{Corps B}

Mat. S. 2.755

2.540

0.215

$\mathrm{II}^{2} \mathrm{O} 45-4.518$

4.334

0.184

$\mathrm{Co}^{2} 90-5.580$

5.080

$\overline{0.500}$

Soit $\mathrm{II}=9.50$

$\mathrm{C}=63.42$

$\mathrm{O}=27.08$

Correspondant à

$$
\mathrm{H}^{9}, 500 \mathrm{C}^{3}, 283 \mathrm{O}^{1}, 692
$$

$\mathrm{H}^{93} \quad \mathrm{C}^{53} \quad \mathrm{O}^{17}$ 
Poids moléculaire. - Cryoscopie.

Pour déterminer le poids moléculaire de l'actéine, nous avons

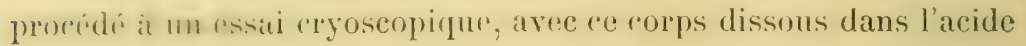
acétique.

Point de congélation de l'ac. acétique cristal............ $\quad \mathbf{1 4}^{0} 80$ d'une solution acétique d'actéine'à $10 \%$. 12060

Différence (abaissement du point de congélation)... $\overline{2^{\circ 26}}$

En substituant dans la formule générale

II

poids moléculaire

$=\mathrm{K} \times \frac{\mathrm{P}}{\mathrm{c}}$ (pourcentage de la solut.)

de la substance)

$$
\begin{aligned}
& \mathrm{P} \text { dans le cas actuel }=10 \% \\
& \text { C } \quad=2^{\circ}, 26 \\
& \text { K } "=39
\end{aligned}
$$

il vient

la formule de l'actéine serait

$$
\mathrm{H}=39 \times \frac{10}{2,26}=172
$$

$$
\frac{\mathrm{C}^{63} \mathrm{H}^{89} \mathrm{O}^{17}}{6}=\mathrm{C}^{9} \mathrm{H}^{14} \mathrm{O}^{3}
$$

Rem. - Nous ne donnons iei les résultats de l'analyse cryoscopique qu'en faisant, à l'égard de leur valeur, les plus expresses réserves.

L'actéine est un glucoside et, pour ce groupe de corps, la détermination, par la cryoscopie, du poids moléculaire laisse place au doute.

L'actéine se présente donc avec les caractères d'un' glucoside, bien différent des glucosides les plus slables, par l'extrème difficulté que l'on éprouve à l'hydrolyser.

Quels sont les rapports chimirues entre l'actéine et le corps B? On pourrait supposer, par analogie, que ce dernier n'est qu'un produit mieux cristallisé, mais identique par sa constitution à l'actéine dont il dérive.

En effet, heaucoup de sulsstances, végétales actives, chimiquement définies, peurent se présenter sous forme cristallisable et sous forme amorphe, et les deux produjts se trouver identiques d'ailleurs comme action et composition. C'est le cas en particulier de la digitaline (1). Le mène fait semble se produire pour le glucoside d'Actra racemosa.

(1) 1889 Adriar et Bandet, Soc. de thérapeut. 
La formule des deux corps semble bien voisine, mais ils difrèrent nettement par certains caractéres (solubilité dans les réactifs, point de fusion).

L'hypothèse qui expliquerait le mieux ses rapports avec le corps B, serait celle-ci.

Dans la plante n'existe qu'un glucoside, l'actéine.

Celui-ci subil très facilement un commencement d'hydrolyse en présence d'agents hydrolysants, même faibles (chaux, oxyde de plomb, acide tartrique), il donne alors naissance à un corps peu différent, le corps B.

Il est, par contre, très difficile d'obtenir (même par les agents énergiques d’hydrolyse) l’hydrolyse complète de lactéine détunblable finalement en glucose, et en un corps que nous n'avons pu encore étudier), qui apparaît alors comme le glucoside le plus stable de tous les glucosides connus.

Celte faculté d'hydrolyse en deux temps n'est d'ailleurs pas si exceptionnelle, chez les glucosides. L'amidon ne présente-t-il pas une série de slades d'hỵdrolyse de plusen plus avancée? Le premier de ces stades, qui abuntit à la production de dextrine el de maltose, est infiniment plus facile à obtenir que les suivants.

Sans insister sur les caractères différentiels, la comparaison la plus superficielle suffit à montrer que l'actéine n'est identirue ni à la berbirine, qui existe dans une Renonculacée : Coptis tectu, ni à la macrocarpine de Thalictrum macrocarpum.

\section{Conclusions}

Tos recherchesnous conduisent, sur presque tous les points, a des conclusions différentes de celles admises justüà ce jour, relativement aux principes actifs d'Acteca racemosa.

Le rhizome (desséché, tel qu'il nous arrive d'Amérique) ne renferme pas trace de principe volatil. Ce principe existe-t-il dans la plante fraîche? C'est un point qui reste à élucider.

Il n'existe pas dans la plante trace de principe alcaloïdique.

Elle renferme une certaine quantité de tanin. 
Il n'existe pas dans la plante deux corps resineux distincts, mais bien un seul et même corps, dont la formule semble bien être $\mathrm{C}^{9} \Pi^{14} \mathrm{O}^{3}$, résineux en apparence, mais en réalilé cristallisable, capable de donner naissance (par hydrolyse?) à un corps, fort peu différent par sa formule, distinct cependant.

Ce corps cristallisable ne peut être en aucune façon confondu avec un saccharose; c'est un glucoside (?) très remarçuable par l'extrême difficulté que l'on éprouve à l'intervertir.

C'est un corps de composition parfaitement définie, et totalement différent de ceux décrits jusqu'à ce jour chez les Renonculacées.

On ne peut lui conserver le nom de Cimicifugine ou de Macrotine, sous lequel on désigne, dans la pharmacopée américaine, le magma résinoüde, précipité par l'eau de la teinture de la plante. Nous proposons pour lui le nom d'Actéine, qui aura l'arantage de rappeter en même temps sa présence (bien probable) dans l'Actée indigène.

Les rapports que peut affecter ce glucoside avec le corps décrit comme résineux ou cristallisable par les chimistes américains sont bien difficiles à établir. Les produits par eux obtenus étaient tous plus ou moins impurs, le corps que nous avons réussi à isoler semble, au contraire, bien défini, au point de vue chimique; la formule de constitution reste à établir.

L'actéine doit exister aussi dans les divers organes d'A. spicata, ce serait le corps résinoïde et glucosidique indiqué par l'analyse élémentaire.

BIBL.

Tilghiman. (Am. J. of Pharmacy, VI.)

DAYIS (G. II.) (Ibid. XXXIII.)

1871. - Conard (T. Elwood) (Ibid.) (Jahresb. Pharm,, 1022). (Journ. de Pharmacie, 29 avril, p. 866.)

1876. - BEACH (L F.) (Ibid.)

1878. - Trimble (F. H.) (Ibid. (4) 50, 468.)

188'. - FalGK (S.) (Ibid. 56, p. 459.)

1884. - » (Pharmaceut. Zeitung, n० 87, p. 749.)

1886. - Liovd (J. U.) and Lloyd (C. G.) (Pharmaceut. Rundschau und Zeitungf. die IVissenschaftl.... New-York, Berlin (C. Springer), n. 2, p. 30).

1886. - Ibid. (Pharmaceut. Zeitung, n²3, p. 185.) 


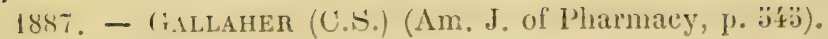

) (Pharmac. Zeitung, no 105, p. 745.

189'. - Dispensatory of the United States of Americal (sevent ed. p. 381-2).

Drugs and Medicines of North America, vol. I. 


\section{CHAPITRE III}

\section{PHARMACOLOGIE - POSOLOGIE}

L'Actiea racemosa est employée en Amérique, et quelque peu en Angleterre, sous des formes pharmaceutiques sur lesquelles il est nécessaire d'insister.

$1^{\circ}$ Racine brute (rhizome). - La poudre du rhizome est d'un emploi peu répandu, elle a été prescrite à la dose journalière de $20 \mathrm{gr}$. (Davis); la dose de 0 grr. 0 ) -0 gr. 20 indiquée par Brminzm (Formulaire clin. de Vienne) ne semble pas en rapport avec les doses prescrites par les praticiens américains. Celles-ci varient entro 1 scrupule et 1 drachme $(1,3-3,9$ gram.)

Si l'on admet, comme nous devons le faire, d'après nos essais chimiques et physiologiques, que le seul principe actif de la plante est l'actéine (la teneur du rhizome en ce corps ne dépassant pas au grand maximum 4 \%), l'administration de 20 grammes de poudre correspond à $0 \mathrm{gr} .80$ d'actéine. Cette dose est susceptible de déterminer des accidents d’irritation gastro-intestinale (V. p. 313). Par contre, la dose de $0 \mathrm{gr}$. 20 de racine brute correspond à la dose insignifiante de $8 \mathrm{~g}^{\mathrm{mm}}$. d'actéine.

La décoction de 1 à 2 grammes de racine pour 100 parties d'eau a parfois été prescrite, à la dose d'un verre à vin toutes les 3 ou 4 heures (Davis).

L'insolubilité complète de l'actéine dans l'eau enlève toute valeur a cette préparation, 'qui ne peut contenir qu'un peu de tannin. Elle est d'ailleurs totalement inusitée aujourd'hui.

$2^{\circ}$ Teinture. - La teinture d'Actée, si utilisée en Amérique, s'obtient en épuisant une partie de rhizome sec par ร̌ ou par 8 (suivant les auteurs) parties d'alcool. Elle est d'une teinte orangée 
claire, d'une saveur quelque peu amère; mélangie à l'eau, elle devient opalescente, par suite de la précipitation de la résine, qu'elle tient en dissolution à la faveur de l'alcool.

Celte teinture contient toute l'actéine du rhizome, gråce à sa parfaite solubilité dans l'alcool. On la prescrit à la dose de XXX à LX gouttes toutes les heures, on toutes les 2,4 ou 6 henres (D.vis); de XXV à XL gouttes trois fois par jour (MAcomsusu); de IV gouttes toutes les deux heures, cost-i-dire à la dose quotidienne de XL it L gouttes (IIEWELkE); de V-L gouttes par jour (Formulaire de Vienne). En moyenne 3-7c; par jour;

Ou sous la forme suivante:

Teinture CLX;

Lau $3 i j$; (PatTon).

3 j.t.d.s.;

Teinture au quart: de XV à LX gouttes par jour, ou V gouttes toutes les heures (Lauden Bruntor).

$3^{\circ}$ Extrait fluide. - Il est presque superflu de rappeler que cet extrait, préparé suivant le procédé américain, représente, poids pour poids, la drogue qui a servi à le confectionner. La dose des principes actifs est la nè̀me dans la drogue et dans l'extrait: mais, dans ce dernier, les matières inertes (insolubles dans l'eau et l'alcool) sont remplacées par un poids égal de dissolvant, mi-partie aqueux, mi-partie alcoolique.

Le temps n'est plus où l'on pouvait croire à l'identité d'action de principes actifs, administrés en poids égal, sous des formes pharceutiques diflérentes. Il importe de se rappeler que, dans les extraits fluides amérieains, la totalité des principes solubles dans l'eau et dans l'alcool se trouvent dissous dans un véhicule mi-partie aqueux, mi-partie alcoolique, additionné d'une cerlaine quantité de glycérine. L'addition de ce dernier corps était, jusqu'à ces derniers lemps, considérée comme n’étant pas de nalure à modifier, d'une manière sensible, les propriétés phrsiologiđques ou thérapeutiques d'une solution médicamenteuse; mais on sait maintenant que 
l'addition de glycérine, alcool triatomique, à une solution de principes arlifs, modifie singulièrement l’équilibre moléculaire de cette solution, affaiblit son activité chimique, et par suite son activité phrsiologique, qui n'est qu une des modalités de la première (1).

Le degré de dilution dune solution médicamenteuse est égralement un facteur important de son aclivité.

Il importe done, pour obtenir des effets comparables, de toujours recourir à des solutions médicamenteuses aussi identiques que possible, sous le triple point de vue de la teneur en substance active, de la dilution et de la nature, ainsi que des proportions relatives, des dissolvants.

Il importe, pour que tout expérimentateur puisse se placer dans des conditions identiques à celles où nous nous sommes placés nous-mème, d'indiquer avec soin la composition exacte de l'extrait fluide par nous employé.

Cet extrait était préparé, suivant le procédé américain, avec le rhizome sec d'A. incemosa, tel qu'il est expédié des drogueries américaines.

L'extrait fluide, préparé selon le procédé américain, et (qui a servi à nos essais physiologiques et cliniques, renfermait pour $100 \mathrm{c}^{3}$ $17 \mathrm{gr}$. d'extrait solide, en dissolution dans de l'alcool à $600 / 0$ environ.

On a prescrit l'extrait fluide à la dose de :

III à XXX gouttes par jour (LAUDER-BRuntoy).

De X à XXX gouttes.

$\mathrm{V}$ gouttes chaque soir (KNoRR).

0 g, 0 马̆ à 0,3 s̆ par jour (Form. de Vienne).

$0 \mathrm{~g}, 75$ par jour dans du sirop de Salsepareille.

(1) Voir pour l'exposé de ces faits :

1879. - Binnandisk ( $7^{\mathfrak{e}}$ Congrès international de Médecine, à Amsterdam).

1896. - Srokvis (B. T.) Leçons de Pharmacothérapie (traduction française, par de Buck et de Moor, Haarlem et Paris (Doin), p. 324). 
En moyenne de 1, 9 a $3,7 \%^{e 3}$ par jour:

Extr. fluide de Cimicifuga

Sirop de Salsepareille. (MilLand).

Sirop simple.

$\mathrm{K}$

Nous avons employé l'extrait fluide, à la dose journalière de X à XL grouttes, dans fï grammes d'eau sucrée. Il se peut qu'au débul l'extrail thuide provoque quelques phénomènes d'intolérance análogues à ceux que déterminent l'actéine (voir plus loin ch. VI) en poudre. Cees phénomènes disparaissent au bout de quelques jours de traitement.

D'après ce que l'on sait de l'àreté des principes volatils contenus dans nombre de Renonculacées, on peut supposer que si la plante fraiche contient réellement le principe volatil, assimilé it une huile essentielle par Divis, elle jouit d'une activité différente de celle de la plante sèche.

Les extraits fluides, préparés en Amérique avec la plante fraîche, utilisés aussitòt, ne seraient peut-être pas comparalles, au point de vue de leur action, à ceux préparés en Europe avec le rhizome sec.

$4{ }^{\circ}$ Résine. - Dans les divers ouvrages récents de pharmacopée américaine, le principe actif est indiqué comme soluble dans l'eau et dans l'alcool. On prépare, sous le nom de Cimicifugine ou Macrotine, un produit impur, d'apparence plus ou moins résinoïde, par précipitation de la teinture alcoolique concentrée de rhizome par addition d'eau. On semble aussi aroir employé en Amérique, sous le nom de Cimicifugine, le prétendu alcaloïde dont nous avons parlé p. 211). Il n'y a pas à parler ici de ce corps introurable). C'est une poudre d'un brun foncé; d'apparence résineuse, faiblement amère, soluble dans l'alcool à $90^{\circ}$, dont la proportion dans le rhizome serait d'environ $40 / 0$ Pakrisil. Les doses prescrites sont assez rariables selon les auteurs: 3, o, $7,10,20,30$ centigrammes en pilules par jour; de 1 à 6 grains dans une pilule avec de la glyceérine. On l'administre, en Amérique, à la dose de $0 \mathrm{gr}, 06: 3$ à $0 \mathrm{gr} ., 13$. 
;"Actèine pure. - Le corps, d'apparence résineuse, que nous avons isolé, et qui est, en réalité, le principe actif, chimiquement défini, y existe dans les proportions de 22,5ั à 29,6 0/00.

La pureté du corps par nous obtenu explique la différence que ces chifles présentent avec ceux donnés par les auteurs américains.

Nous avons primitivement employé l'actéine en poudre, en cachets ou en pilules.

Les pilules contenaient 30 centigrammes chacune d'actéine. Nous les donnions à la dose journalière de 5 à 6 pilules (soit de 5 à 30 centigrammes), en augmentant progressivement de 1 par jour.

Les cachels étaient primitivement de $\ddot{j}$ centigrammes chacun (2 par jour, soit 10 centigrammes), puis de 15 centigrammes 2 par jour, soit 30 centigrammes. L'ingestion des cachets ayant donné lieu à des phénomènes d'intolérance, nous avons renoncé à leur emploi. L'emploi de pilules contenant une même dose d'actéine n'est au contraire suivi d'aucun phénomène fàcheux. Le fait s'explique facilement : la masse pilulaire offre une certaine résistance à l'action dissolvante des sucs digestifs, et le médicament n arrive que peu à peu au contact de la muqueuse des voies digestives. On peut même se demander si la désagrégation des pilules dans le tube digestif est complète.

L'administration de l'actéine en cachets est tout à fait à rejeter ; avec les cachets une quantité trop considérable (même si on mélange l'actéine avec une substance inerte, telle que le sucre de lait) de ce corps irritant est mise en liberté dans la cavité stomacale.

De toute façon, l'actéine en cachets nous parait ne pouvoir être maniée qu'avec la plus grande prudence.

On pourrait préparer une solution alcoolique d'actéine, contenant par c $c^{3} 1$ milligramme d'actéine. Bien que facile à manier, au point de vue thérapeutique, cette préparation nous a paru très inférieure a la solution alcaline du principe actif. Elle ne se prête d'ailleurs en rien à l'expérimentation physiologique. 
La solution alcaline d'actéine, que nous avons employée dans nos essais physiologiques et cliniques, a été préparée de la façon suivante :

13 grammes d'actéine, finement pulvérisée, sont dissous dans $30^{\text {c3 }}$ d'alcool à $90^{\circ}$. L'addition d'eau à cette solution alcoolique y détermine un précipité partiel. Ce précipité est redissous par l'addition d'une solution sodique (a $\ddot{3} \%$ environ). On ajoute à nouveau de l'eau, et on redissout le précipité formé, jusqu'à obtention de $300^{\text {c3 }}$. La solution est alors chaufée au bain-marie, pour chasser l'alcool. Du fait du départ de l'alcool, on obtient à nouveau une précipitation notable. On fait passer un courant de $\mathrm{Co}^{2}$ jusqu'à refus, pour saturer la soude libre, une nouvelle précipitation d'actéine se produit. On filtre, et, dans le liquide limpide, on dose l'actéine dissoute et le carbonate de soude. (Pour ce dosage, on évapore ì sec, et on pèse le résidu; de ce poids total, on retranche le poids du carbonate de suude, après destruction de l'actéine par calcination.)

Dans cette solution, l'alcali libre est entièrement neutralisé, il n'existe plus que des traces insignifiantes d'alcool, et chaque $c^{3}$ contient 0 gr. 02 d'actéine solubilisée, et $0 \mathrm{gr}$. 03 de carbonate de soude.

C'est un liquide limpide, jaune paille, à saveur franchement piquante (carbonate de soude), fraîche, puis d'une amertume légère (actéine) et persistante, présentant quelque analogie avec celle des solutions faibles de sulfate de quinine à odeur forte et spéciale.

D'après le nombre de $\mathrm{e}^{3}$ de cette solution, administrés au malade, il nous est facile, dans les essais cliniques, de savoir exactement la dose d'actéine solubilisée qu'il reçoit journellement. Nous évaJuerons toujours cette dose en centigrammes. (V. les observ. du chap. VI.)

L'addition d'acides à la solution alcaline d'actéine provoquant sa précipitation plus ou moins complète, il est intéressant de savoir. si 
- celte précipitalion se produit dans l'estomac, au contact du suc gastrique.

\section{L'expérience suivante répond à cette question :}

Sur la solution alcaline, ci-dessus décrite, on essaye l'action d'un suc gastrique artificiel, contenant $2 \%$ d'HCl. En présence d'un excès de ce suc gastrique artificiel, lit solution ne précipite que très faiblement, et d'une façon pratiquement négligeable.

Donc la solution alcaline d'actéine ne précipite pas dans l'estomac, il en est évidemment de mème au contacl des sucs intestinaux alcalins.

Ce fait nous amène à cette conclusion, que l'actéine ainsi administrée reste solubilisée dans toute la longueur du tube digestif, et, par suite de son absorption possible, peut passer dans la circulation générale. L'actéine administrée en poudre, au contraire, ne doit ître que bien imparfaitement soluble dans des sucs digestifs alcalins, et agir surtout par action locale sur le tube digestif.

Celle solution se prête fort bien à des essais physiologiques; elle ne précipite pas en présence du sang alcalin, el contient (en chiffres ronds) $30 \%$ de carbonate de soude, sel on ne peut moins toxique. Le sang normal contenant environ $7 \%$ de sels solubles, on roit qu'en étendant la solution alcaline primitive de quatre fois son volume d'eau, on obtient presque unesolution d'actéine (très diluée, il est vrai) dans du sérum artificiel.

La présence du carbonate de soude dans les proportions indiquées) dans la solution ne paraît pas de nature à modifier aucunement l'action propre de lactéine. Les effets physiologiques ou thérapeutiques observés se rapporteront bien à ce dernier corps, étant donné le peu de toxicité et d'activité physiologique du carbonate de soude.

Dans la médication alcaline, on ne dépasse guère, d'une façon courante, les doses journalières de $\tilde{j}$ grammes de bicarbonate de soude et de $2 \mathrm{gr}$. Јे de carbonate. 
Par excès de prudence, nous avons commencé nos essais sur l'homme par des doses journalieres de $2 / 10$ de e.3 par jour. Yous n'arons jamais dépassé la dose journalière de for e de la solution alcaline dactione les 60 ) centigr. d'achine qu'elle contient étant susceptibles dr provorfuer des malaises). Inone, à eelle dose maxima, notre solution n’introduisait jamais dans l'organisme des malades guère plus de I sramme de carbonale de soude par jour. En règlo générale, les doses par nous employées ne dépassant pas 20 c c de $^{3}$ solution. le malade n'ingérait que 60 centigrammes de carbonate, dose négligeable au proint de vue des résultats thérapeutiques.

Chez l'homme, les phénomènes d'intolérance se produisent, presque dès le premier jour du traitement, lorsqu'on administre $30^{\text {e3 }}$ d'actéine pulrérulente, au bout de deux ou trois jour's lorsque l'on se borne a des doses de 10 centigrammes. L'extrail tluide peut aussi occasionner quelques acridents analogues, mais qui s'alténuent après un usage de quèlyues jours. L'actéine en solution sodique est, au contraire, toujours bien tolérée, à la dose journalière de ô à 6 centigrammes, dilués dans 4 ğ grammes d'eau.

V. pour la posologie, dans chaque affection, les chiffres indiqués par les auteurs (chap. VI).

BibL. - Outre les sources citées plus haut (chap. II), oủ il est incidemment question de la posologie, v. Bibl. chap. VI et:

1894. - The dispensatiry of the Unit. Stales of America (sevent. ed. p. 381.)

1895. - Martindale (W.) and Westcott (W. Wrin). The Extra Pharmacopoeia, (eight ed. London.) 


\section{CHAPITRE IV}

\section{L'AGTAA SPICATA AU POINT DE VUE TOXICO- LOGIQUE ET MÉDICO-LÉGAL}

\section{A. - Intoxications spontanées.}

D'après l'opinion généralement admise, toutes les parties de l'Actica spicata sont vénéneuses, mais les baies et le rhizome sont les parties les plus vénéneuses. La dessiccation leur enlèverait une partie de leur toxicité.

Cette assertion donnerait a supposer que, comme pour beaucoup de Renonculacées, l'Actée contiendrait un principe toxique volatil.

L'analyse chimique nous a montré la fausseté de cette hypothèse.

Les moutons, les ânes, les chèvres, mangeraient l'Actée verte, sans en paraitre incommodés (Bulliard). LinNÉ déclare les fruits de l'Actée capables de déterminer un délire furieux, suivi de mort. Tous les auteur's, à la suite de Linné, déclarent la plante fort dangereuse.

LEMERGier aurait vu ses baies produire une sorte d'ivresse, des troubles cérébraux et de l'irritation gastro-intestinale; un des malades par lui observés présenta des vomissements.

"Il faut bien prendre garde qu'on en use intérieurement, car cette plante est un poison, comme l'aconit ordinaire. "(Dict. de mat. méd.)

La plante fraiche tue les canards et les poules (Mérat et Delens, Bulliard). Son extrait serait également toxique pour ces oiseaux (Lemonnier). Cependant, d'après Mattei, les baies d'Actée seraient disséminées par les oiseaux frugivores. (Bien qu'ayant observé pendant plusieurs années cette plante, dans les forèts où elle est fréquente, nous n'avons jamais pu vérifier le fait.)

L'ingestion des baies a été, dans un cas observé par Colden, suivie de malaises, de sueurs froides, sans autre accident. 
La teinture préparée avec la racine n'a déterminé, dans un cas observé par Colden, qu'un malaise, accompagné de sueurs froides, sans autre accident.

Onfila déclare avoir fait souvent prendre 4 à 6 onces de décoction d'A. spicata, cueillie en mai, sans avoir observé aucun phénomène fácheux.

Les empoisonnements spontanés par l'Actée sont rares, semblet-il. Tout au plus, dit-on, les enfants pourraient-ils manger les baies, mais l'odeur désagréable de la plante doit suffire, en général, à les en écarter.

"Ne laissez jamais les fruits de cette plante, dit BullianI, dans l'état de maturité, après les tiges, parce que les enfants, naturellement curieux, peuvent en manger et s'empoisonner. ")

Lo rhizome d'Actée est, dans certaines campagnes, employé comme purgatif, et ses autres parties comme parasiticides; quelques empoisonnements ont pu se produire de ce chef.

“ Cette plante occupe un des premiers rangs parmi les plantes âcres : dans des mains peu excrcées, son usage peut être très dangereux. " (BulliaRd.)

On a rapproché les symptômes de l'intoxication par l'Actée de ceux que provoque l'intoxication par les Hellébores. Inflammation vive du tube digestif, purgation violente, vomissements, dépression du système nerveux, puis ivresse et délire furieux, tel serait le tableau général de cette intoxication.

BıBL. 1737. -- LinNÉ. Flora Lapponica, Amstelodami, $8^{\circ}$.

1743. - Colden. Act. Upsal, p. 132.

1798. - Bulliard. Hist. des pl. vénén. et suspect. de la France, $2^{*}$ éd., pp. 154-6, in- $8^{\circ}$, Paris.

Lemercier (de Rochefort). (Compte rendu de la Soc. méd. de Lyon), cité dans

1868. - CAzIN (F.-J.). Traité prat. et raison. des pl. médicin. indigènes, p. 29.

1888. - Mattei (Boll. del Naturalista).

L'affirmation de Bracoxiot que l'Actée n'a rien d'âcre au goût, est certainement exagérée, sa saveur et son odeur sont plutôt désagréables. 
Tous n'avons vu survenir chez nous-mêmes, et chez le chien, aucuu simptome fitcheux a la suite de l'ingestion de quelques baies mûres d'Actée.

Que des enfants, trompés par leur saveur un peu âcre, mais en même temps sucrée, et leur couleur, soient tentés de consommer ces fruits, la chose ne semble pas douteuse; mais, étant donnée la faible quantité de corps résinoïde (actéine) qu'ils contiennent, et labsence de tout autre principe toxique, il futudrait qu un nombre fort considérable de fruits fussent ingérés avant de déterminer des phénomènes dintoxication. Ceux-ci seraient du même ordre que ceux que nous avons observés (p. 312) dans l'intoxication expérimentale avec l'actéine.

La proportion plus considérable de corps résinoïde contenue dans le rhizome permet de comprendre que son usage, comme purgatif, puisse déterminer, à dose un peu forte, des phénomènes de gastro-entérite aiguë, peut-être mortels dans certains cas.

Ii semble qu'en aucun cas l'Actée ne se prête facilement à être employée comme toxique dans un but criminel. Tout au plus pourrait-on supposer que, dans les campagnes où l'usage de l'Actée comme purgatif est établi, on pourrait faire intentionnellement prendre une dose trop forte de poudre ou de décoction de rhizome.

Les symptômes et les lésions que déterminerait, chez l'homme, l'intoxication par l'Actée, doivent ètre très semblables a ceux décrits à propos de l'intoxication expérimentale. Y revenir ici est chose inutile. Remarquons seulement que l'ivresse et le délire furieux, indiqués par les auteurs comme symptômes dominants de cette intoxication, semblent par analogie avec ce qui se voit chez les animaux) devoir faire défaut chez l'homme.

\section{B. - Détermination botanique médico-légale.}

Le médecin légiste n'aura jamais à se prononcer que sur le fruit. et le rhizone de l'Actée. S’ils sont entiers, leur détermination sera facile, d'après les caractères botaniques précédemment décrits. 
La poudre de rhizome, ou les fragments infusés de ce dernier, les débris de periearpe, seront tomjours detrminables arece précision par un examen histologique, dont les résultats seront comparés à ceux que donne l'étude anatomique et histologique du rhizome et du fruit.

La recherche des graines dans le tube digestif, à l'autopsie, permeltra toujours de distinguer l'Actéc des autres fruits noirs ou vionlacés que l'on peut y rencontrer. Ces graines, traversant le tube digestif sans altérations, pourront être déterminées, soit par leur's caractères extérieurs, soit par leur structure microscopique.

\section{G. - Recherche chimique du principe toxique.}

L'absorption de l'actéine est assez lente el faible, pour que sa recherche dans le tube digestif puisse être fructueuse, si elle s'y trouve en assez grande abondance.

Il ne semble pas que ce soit d'arec les substances résineuses (aloès, myrrhe, jalapine, convolvuline, elc.), que l'actéine doive être distinguée, dans une recherche toxicologique, mais plutôt d'avec les glucosides tels yue l'helléborine et l'hellij.oréine. La confusion avec cette dernière pourrait être rendue plus facile, à cause de la solubilité des deux corps dans l'alcool et le chloroforme; tandis que l'helléboréine est soluble dans l'eau et peu soluble dans l'alcool. Nous arons vu que la coloration déterminée par laction de $\mathrm{SO}^{\prime} \mathrm{H}^{2}$ sur ]'actėine est brune; l'helléborine, au contraire, se colore en violet par ce réactif, et l'helléboréine en rouge foncé.

Il semble que, dans toute intoxication surtout dans les cas ligers qui sont les plus fréquents) par l'Actée, l'analyse botanique est appelée à domner des résultats bien supérieurs, en rapidité et en précision, à ceux que peut fournir l'analyse chimique. 


\section{CHAPITRE V}

\section{ÉTUDE PHYSIOLOGIQUE}

\section{A. - Actæa spicata.}

Le rhizome et le fruit de l'Actée indigène sont généralement réputés toxiques. Aucune expérience précise n'a cependant été entée, jusqu'ici, relativement à cette toxicité.

Orfila déclare avoir fait souvent prendre 4 à 6 onces de décoction d'Actren spicata, cueillie en mai, sans avoir observé aucun phénomène fâcheux. Il y a loin de cette observation à l'assertion de Linsé, qui aurait pu s'assurer, par plusieurs exemples, que les baies d'A. spicata sont un poison violent, qui fait périr rapidement les chiens. Elles détermineraient un délire violent, suivi de mort.

C'est à cette opinion que se sont ralliés la plupart des auteurs, sans apporter d'ailleurs aucune preuve expérimentale en sa faveur.

L'ingestion des baies d'Actée (péricarpe et graines) ne détermine, chez le chien, aucun symptome appréciable d'intoxication (même à la dose de 12 à 15 à la fois).

L'analyse chimique (v. p. 206) n'y révèle d'ailleurs qu'un seul principe que l'on peut à priori supposer toxique: le corps résineux, qui n'existe dans le fruit qu'en petite quantité, et manque totale. ment dans les graines.

Les essais, tentés pour rechercher les alcalö̈des que l'on pourait supposer exister dans les fruits d'Actæa spicata (v. p. 209), ont été complétés par quelđues essais physiologiques, négatifs d'ailleur's.

10 L'extrait aqueux de fruits (péricarpe + graines) est traité par la potasse, puis agité avec de l'éther. 
2 L'extrait aqueux a été précipité par le sous-acétate de plomb et filtrí; on débarrasse le liquide filtré de l'excès de ploml, par un courant de I'S'S, on filtre, on concentre dans le vide, puis on agite avec de l'éther. L'éther de ces deux essais, décanté, donne après évaporation un résidu très faible. Ce résidu bien neutralisé est injecté à la grenouille sans déterminer le moindre accident

Donc, les fruits d'Actea spicata ne renferment, ni dans leur péricarpe, ni dans leurs graines, de principes alcaloüdiques toxiques.

L'extrait alcoolique, tenant en dissolution la résine, se borne it produire, après injection sous la peau à la grenouille et au cobaye, un peu d'inertie passagère.

La tris grande analogie (pour ne pas dire identité) entre le corps d'apparence résinoïde qui existe dans l'Actéc spicata, et l'acléine extraite de l'Actáa racemosa, nous a engagé à porter nos efforts sur cette dernière substance, et nous ne relatons ici ces quelques essais, avec l'Actée indigène, que pour montrer la toxicité extrêmement faible de cette plante, employée en nature.

\section{B. - Actæa racemosa.}

I.es essais physiologiques tentés avec l'Actxa racemosa se réduisent ì une courte note d'Hutcursson; nous en analyserons les résultats, en les comparant à ceux que nous ayons personnellement obtenus.

Nos essais ont été tentés avec: $1^{\circ}$ l'extrait fluide; $2^{\circ}$ l'actéine en poudre; $3^{\circ} l^{\prime}$ actéine en solution alcaline.

Nous allons d'abord examiner la marche générale de l'intoxication, puis chercher à élucider l'action particulière de l'Actée sur les diverses fonctions.

EXPÉRIENCES AVEC L'EXTRAIT FLUIDE

Nous ayons indiqué plus haut (v. p. żz) la composition exacte de l'extrait qui a servi à nos expériences. Nous ferons encore remarquer qu'à priori l'action de cet extrait peut différer de celle attribuée ì l'extrait préparé avec la plante fraiche, tel qu'il a pu être expérimenté en Amérique. La contradiction qui existe, sur plusieurs points importants, entre nos conclusions et celles précédemment formulées par Hutchissox, s'explique peutêtre par une différence de 
anmposition entre les extraits employés par cet expérimentiteur et frar nous. Même préparés tous deux avec la plante sèche, ces deux extrails peurent peut-être présenter une teneur assez différente en alcool:

\section{Gienouille.}

Pour II thinison, XXX goultes d'extrait fluide injectées dans le sac lymphatique postérieur d'une grenouille, de taille moyenne, suffiraient à amener la mort. Quelques minutes après l'injection, la paralysie de tous les muscles se déclarerait, en même temps qu'une anesthésie générale; l'animal présente une diminution progressire des mouvements respiratoires, et se " cyanose ". (?)

E.xp. I. - Grenouille vivace, neuve, poids.... $22 \mathrm{gr}$.

$\mathrm{T} . \ldots \ldots \ldots 15^{\circ}$

4 h. 30. - XXX Gouttes d'extrait fluide dans le sac lymphatique dorsal.

4 h. 40. - Notilité intacte, soubresauts de tout le corps, à intervalles réguliers; ces soubresauts rappellent asssez (bien qu'avec une intensité beaucoup plus faible) les soubresauts convulsifs que l'on observe à la moindre excitation dans l'intoxication par la strychnine.

4. h. 45. - A cette période primitive d'excitation succède une deuxième période de dépression; sensibilité (tactile et thermique) des téguments et de la cornée intacte; diminution du nombre et de l'amplitude des mouvements respiratoires; longues périodes d'apnée.

5 h. - Addition latente des excitations, temps perdu dans la réponse à ces excitations.

$\ddot{~ o ̈ ~ h . ~ 1: 3 . ~-~ R y t h m e ~ r e s p i r a t o i r e ~ a s s e z ~ r e ́ g u l i e r, ~ m a i s ~ m o u v e-~}$ ments d'une amplitude presque nulle, mouvements volontaires rares et incoordonnés; abolition de tout réflexe rotulien, sensibilité thermique totalement disparue, sensibilité tactile très diminuée, inertie à peu près complète.

1 h. $1 / 2$. - Le lendemain, près de 20 heures après l'injection, la grenouille est encore vivante, mais en état de coma.

马 h. - Mort.

Autopsie immédiate: Légère congestion des vaisseaux abdo. minaux; tube digestif, foie, rein normaux; cœur répondant encore aux excitations mécaniques, en diastole, gorgé 'de sang noir non coagulé; poumons très rétractés, gorgés de sang noir. 
Exp. II. - Deux autres expériences, tentées dans des conditions iden tiques, donnent les mêmes résultats.

Exp. III. - L'injection de XL gouttes d'extrait fluide amẻne la mort, plus rapidement (au bout de 8 heures), en déterminant les mèmes symptômes d'intoxication et les mèmes lésions.

L'extrait thuide détermine done une première perciode d'excilabilité, qui avait échappé à l'attention; puis survient une seconde période de dépression, qui ne se termine que par la mort. Le rylhme respiratoire est influencé avant la molricité et la sensibilité; la paralysie progressive dez différents muscles n'est due it la localisation du poison, ni sur les plarues motrices, ni sur la fibre musculaire, car, jusqu'à l'établissement de la période définitive de coma, les mouvements volontaires sont possibles.

L'action du toxique parait surtout se localiser sur la moelle; il est, en effet, assez facile de s'assurer de l'intégrité, au cours de l'intoxication, des terminaisons nerveuses sensitives, ainsi que des nerf's moteurs.

Exp. IV. - On isole, par une ligature, un des membres postérieurs à sa racine, en laissant le sciatique en dehors de la ligature. L'extrait fluide est alors injecté dans la partie antérieure du corps. L'excitation (à l'aide d'un courant induit d'intensité médiocre) détermine une réaction motrice d'égale intensité, que cette excitation porte sur le membre isolé, soustrait par suite à l'action du toxique, ou sur l'autre membre. Lorsque l'intoxication est plus avancée, la parésie est aussi nette dans les deux membres.

Exp. V. - Une autre grenouille, préparée de la mème façon, reçoit de l'extrait fluide dans le membre isolé, ce membre ne perd ni sensibilité ni contractilité; il en est de mème du reste du corps.

Done, à dose relativement considérable, l'extrait fluide détermine une diminution d'excitabilité des centres médullaires; cette diminution n'est pas sous la dépendance d’une excitabilité exagérée des centres encéphaliques, capable d'exercer une action inhibitoire sur les centres médullaires, car elle persiste chez une grenouille à bulbe sectionné. 


\section{Influence sur la respiration.}

L'influence de l'Actée sur le rythme et l'amplitude de la respiralion pent itre mise en evidence, chez la grenouille, par l'enregistrement graphique des pulsations du plancher buccal, pulsations qui traduisent les mouvements de déglutition de l'air.

Exp. VI. - Pour enregistrer graphiquement ces pulsations, il suffit d'appliquer sur le plancher buccal une mince lame d'aluminium, taillée sur la forme de ce plancher et reliée, par une mince tige de même métal, au levier d'un tambour manipulateur de Marey. Les déplacements imprimés à la membrane de ce tambour sont transmis à un tambour ins. cripteur, de faible capacité et à longue plume inscriptrice (d'où grande sensibilité de l'appareil).
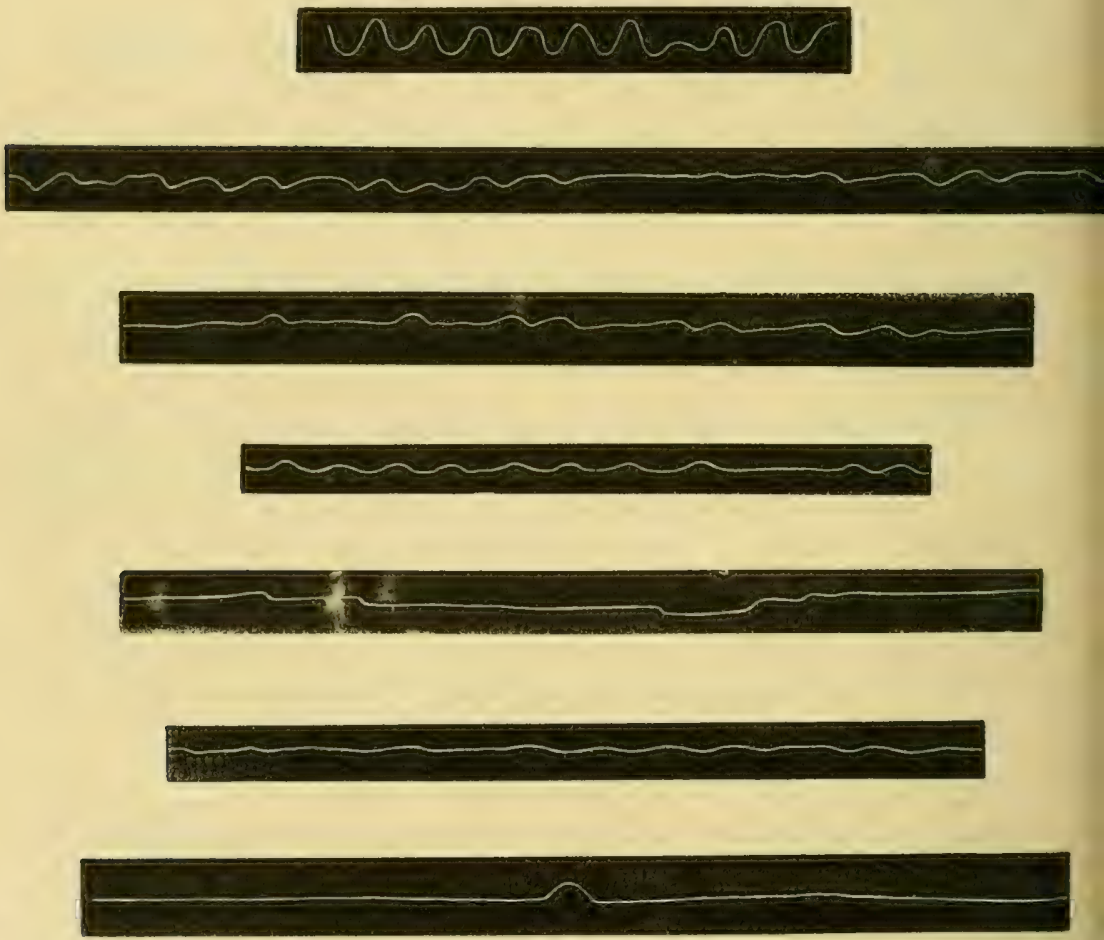

Fig. 67. - Modifications des pulsations buccales chez la grenouille (mouvements de la déglutition) au cour's de l'intoxication par l'extrait fluide d'Actée. (Expér. VI.)

(Phot. directe, sans réduction.) 
Une grenouille du poids de 30 grammes reçoit dans le sac lymphatique dorsal XXX gouttes d'extrait fluide. Les tracés ci-contre montrent nettement les modifications des mouvements respiratoires i mesure que l'intoxication s'accentue (fig. 67).

Les mouvements de deghlutition sont normalement quelque peu arythmiques (I); dès le début de l'intoxication cette arythmie s'accentue, leur amplitude diminue, et des périodes plus ou moins longues de pause respiratoire surviennent (III). Par instant, le rythme normal se rétablit, mais il n'en est point de même de l'intensité (IV). Il peut mème survenir (V) une véritable tétanisation, de plus ou moins longue durée, des muscles du plancher buccal qui se trouve alors rétracté; cette rétraction se traduit sur le tracé par une descente en plusieurs temps; puis brusquement la contraction cesse et le plancher revient à sa tension normale; finalement les mouvements respiratoires ne se traduisent plus que par des ondulations insensibles; la ligne droite qui indique leur cessilion n'est plus interrompue qu'à de rares intervalles par une ondulation nette, ébauche d'une tendance, vite avortée, à la reprise des mouvements (VII).

\section{Influence sur le cour.}

L'extrait fluide agit-il sur les mouvements cardiaques? H'tchissox aftirme que le cœur de la grenouille, détaché sur l'animal normal et placé dans l'extrait pur, ralentit aussitôt ses battements: après quelques contractions, il s'arrête en diastole. Si le contact avec le poison est prolongé, cet arrêt est définitif; mais le cœur, enlevé après quelques minutes de contact, reprend ses contractions sous l'influence d'une excitation directe.

Selon le même auteur, une goutte d'extrait tombant sur le cœur, mis à nu, mais ayant conservé toutes ses connexions, suffit à l'arrêter en diastole.

Nos expériences ne confirment point cette manière de voir.

Exp. VII. - Sur une grenouille, le cœur est mis à nu, et ses contractions enregistrées par le cardiographe Verdin-Vilert (fig. 68). 
L'instillation de plusieurs gouttes ne provoque que des modifications passagères de la forme de la contraction curdiaque, le rytlıme reste très sensiblement le même. Lors de la tombée de chaque goutte, par suite de l'irritation directe du myocarde (due peut-ètre simplement a la présence de l'alcool), le ventricule blanchit et modifie sa contraction, puis celle-ci revient au type normal.

$\mathrm{Si}$, à des intervalles très rapprochés, on projette sur le coeur l'extrait goutte à goutte, l'amplitude des contractions diminue progressivement et avec rapidité; mais, au bout d'un temps relativement très court, les contractions reprennent une amplitude, supérieure mème à la normale, et leur rythme, bien que d'abord ralenti, se rétablit peu à peu pour revenir bientôt à la normale.

L'influence de l'extrait est donc relativement bien faible.

Pour obtenir des modifications notables dans la forme de la systole ventriculaire, il faut employer des doses, relativement fortes, à intervalles très rapprochés, le rythme ne se modifie à aucun moment, et l'amplitude ne tarde pas à revenir à la normale.

Même sur un cœur déprimé, le contact de plusieurs gouttes d'extrait relève notablement, instantanément et temporairement la force de la contraction (V).

Un cœur de grenouille, qui a subi le contact direct de 20 gouttes d'extrait fluide, bat encore, avec même rythme et même amplitude 4 heures après la cessation de l'expérience.

On peut donc conclure que l'extrait fluide n'agit, contrairement à l'opinion d'Hutchisson, ni sur le myocarde ni sur les ganglions intracardiaques; les faibles modifications qu'il imprime à la contraction ne sont guère attribuables qu'à l'irritation locale temporaire qu'il détermine, irritation due en grande partie à l'alcool qu'il renferme.

\section{Cobaye.}

Par vois stomacale. - L'administration d'extrait fluide, par

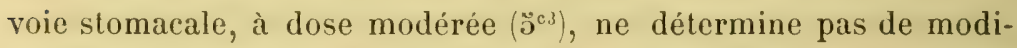
fication physiologique notable chez le cobaye. Une dose massive de $10,15 \%$, chez un cobaye de poids moyen, détermine l'apparition brusque d'un état comateux, dont l'alcool doit être la principale cause; l'animal ne meurt qu'au bout de plusieurs heures, ct les 
lésions constatées sur le tube digestif sont, à l'intensité près, celles que nous étudierons en détail, à propos de l'intoxication par l'actéine. Inutile donc d'y insister ici.

Par voie hypodermique.

Exp. VIII. - Cobaye mâle, neuf; poids : $48 \ddot{\text { grammes. }}$

2 h. 30. - Sous la peau du flanc, àc3 d'extrait. L'injection n'est point douloureuse.

2 h. 40.- Quelques minutes après l'injection, l'animal est étendu inerte sur le côté, sa respiration est régulière, mais aflaiblie; progressivement la sensibilité tactile et thermique diminue jusqu'à disparaître. Lorsqu'une excitation unique est impuissante à déterminer une réaction, on peut encore, par une suite d'excitations, déterminer, après addition latente, une réaction de peu d'intensité.

4 h. - Cet état se prolonge jusqu'à six heures, à ce moment la sensibilité cornéenne est elle-mème fort diminuée; la température qui a diminué progressivement, lepuis le commencement de l'expérience, n'atteint plus que $32^{\circ}$, la respiration cesse peu à peu d'être perceptible, le cœur n'a plus que des contractions fibrillaires, la mort survient à 4 h. 40 .

Les modifications des mouvements respiratoires sont très analogues à celles qui seront étudiées plus loin, à propos de l'intoxication par l'actéine. L'autopsic ne révèle que des lésions peu accentuées, le fait le plus saillant est la congestion des viscères abdominaux et des poumons.

Nous insistons peu, d'ailleurs, sur les symptômes déterminés par. l'intoxication à l'aide d'extrait fluide, à cause de la proportion assez forted'alcool contenue dans cet extrait, et de la sensibilité des rongeurs à l'alcool.

\section{Lapin.}

Par voie stomacale. - L'intoxication par voie stomacale, chez le lapin, reproduit trop exactement le tableau de lintoxication chez le cobaye, pour qu'il soit besoin d'insister. Le lapin est cependant plus sensible que le cobaye.

Par voie intraveineuse. - L'injection, dans la veine auriculaire, de $3^{\text {c3 }}$ d'extrait détermine instantanément la mort, après une grande convulsion généralisée. La sensibilité de l'endocarde du lapin et la 
toxicite fort grande de l'alcool pour cet animal enlevent, d'ailleurs, presque tout intérêt à l'essai de l'extrait lluide par voie intra-veineuse. Toutes les modifications observées sont attribuables à une action directe de l'alcool sur le myocarde.

Par voie péritonéale. - Il n’en va pas de même avec l'injection par voie péritonéale. A forte dose, l'extrait injecté dans cette séreuse, pourrait ètre accusé de produire, par voie purement réflexe, des modifications notables des grandes fonctions. En se bornant, au contraire, à dis doses faibles, on peut faire pénélrer le toxique dans la circulation générale, avec une rapidité suffisante, pour observer avec précision les modifications respiratoires et cardiacues. Les premières sont à peine appréciables.

Influence sur la contraction cardiaque.-L'examen de la contraction cardiaque, pendant la durée de l'intoxication par une dose faible, est, au contraire, capable de nous révéler certains faits intéressants.

Exp. IX. - Le tracé cardiographique d'un lapin, neuf, adulte, de poids moyen $(2 \mathrm{k}, 300)$, est recueilli i l'aide du cardiographe Bardier.

2 h. 3ă. - 303 d'extrait dans le péritoine. Quelques minutes après, une arythmie très nette commence à se manifester (III); mais le nombre des pulsations cardiaques reste le même, tandis que leur amplitude dininue (II, III).

3 h. - L'amplitude est presque revenue à la normale (IV).

4 h. - Nouvelle injection dans le péritoine de $3 \mathrm{c}^{3}$, l'amplitude diminue aussitôt (V), l'arythmie réapparait (VI) et le tracé, pris d'une facon ininterrompue jusqu'i 4 h. 30 , met en évidence une arythmie des plus curieuses, se reproduisant à intervalles réguliers; pendant de courtes périodes (une seconde environ), séparées par des périodes plus longues où la contraction est normale, bien que l'amplitude soit plus faible.

Pendant les périodes d'arythmie, la forme de la contraction cardiaque mérite d'être analysée avec quelques détails.

Pendant les périodes de rythme normal (I), la portion de la courbe correspondante à la ligne de descente présente deux crochets, le second bien moins accusé que le premier. Le premier crochet est dû à la fermeture des valvules aortiques, et le second à la fermeture des valvules de l'artère pulmonaire. 

Pendant la periode d'arythmie perpiodigur VIII), dans chaque pulsation, le rochet de descente en considirablement plus areusé, et le ressaut do la ligrue de deseente sur e point arrive à alteindre près de la moitic de la hauleur de la systole ventriculatire. Ia prointe de la systole, au contraire. lend à clisparaitre presque completement.

L'interprétation de cette modification est évidemment fort délicate, il nous semble cependant qu'elle pourrait être donnée de la façon suivante :

La contraction ventriculaire étant moins énergique, la pression diminue dans l'aorte, et le claquement des valvules sigmoïdes se produit avec moins d'énergie.

Nous verrons, d'ailleurs, plus loin, par l'étude des modifications de la pression sanguine, que sous l'influence de l'extrait celle-ci tombe au-dessous de la normale (diminution du tonus vaseulaire).

Si on admet qu'il existe un ralentissement ou une gêne dans la rirrulation pulmonaire, la pression sanguine doit augmenter ausi dans l'artère pulmonaire, d'où fermeture plus brusque des valvules artérielles, et ressaut plus.intense au bas de la ligne de descente de la systole rentriculaire. Ce tracé n'est pas sans présenter une certaine analogie a l'intensité près) avec celui de l’insuffisance. mitrale.

L'explication ci-dessus (que nous ne proposons évidemment qu'avec réserve, mais qui présente l’incontestable mérite d'expliquer les faits d'une maniere satisfaisante), conduirait à admettre que lespériodes d'arythmie seraient contemporaines de périodes de vaso-contriction pulmonaire, autrement dit, qu'il y aurait intervention d’un phénomine nerveux se reproduisant à intervalles réguliers.

Il est intéressant de chercher, si, à doses très faibles, l'extrait fluide, injecté directement dans une veine, est susceptible d'imprimer quelque modification à la contraction cardiaque. 
Exp. X. - Un lapin de poids moyen (3 k.).

4 h. - Recoit dans la veine auriculaire V gouttes d'extr. fluirle.

(Sa contraction cardiaque est enregistrée par le cardiographe Bardier.)

4 1. 2. - Diminution légère de l'amplitude (II).

4 h. 15. - Il y a un retard très net dans l'apparition du crochet de descente (III).

4 h. 20. - Diminution légère de l'amplitude (IV).

4 h. 30. - L'amplitude revient it la normale (V).

4 h. 35. - Très légère tendance à de l'arythmie (VI).

Fig. 70. - Modifications de la contraction cardiaque, chez le Lapin, au cours de l'intoxication par l'extr. lluide d'Actée (voie intraveineuse). (Expér. X) (Cardiogrammes fournis par le cardiographe. Bardier). - I. 'I'race normal. La vitesse du cylindre enromstrent est un peu plus faible que dans l'expér. précédente). Le second crochet de la ligne de descente n'est pas enregistré, parce que la pointe exploratrice n'affleure pas exactemênt à la pointe du coeur. L'animal donne normalement des contractions plus fortes que celui de l'experience precédente.

(Photog. directe, sans réduct.)

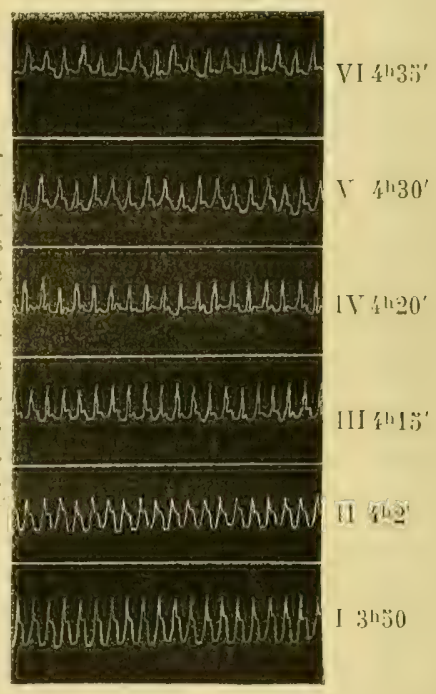

L'injection d'une dose très' faible d'extrait dans une veine ne modifie en rien le rythme cardiaque; il ne diminue l'amplitude de la contraction que d'une facon toule temporaire et peu accusée. Le retard dans la fermeture des valvules sigmoïdes s'expliquerait par une diminution de pression artérielle, due à une vaso-dilatation. Somme loute, l'extrait à très faible dose, même en injection intraveineuse n'agit en rien sur le rythme du coeur, et fort peu sur la force des contractions.

\section{Chien.}

Influence sur la pression artérielle. — Les essais sur le chien séprê- 
Arec une dose modérée d'extrait, la chute de la pression n'est donc que tout ì fait temporaire.

Rem. - Notons, en passant, que pendant la durée de l'intoxicalion la coagulation du sang est quelque peu retardée. Le retard apporti a la formation du caillot est de 10', lorsque la pression est encore it un chiffe inférieur, de ösculement lorsqu'elle remonte à son chiffre primitif.

La cause de ce phénomène accessoire réside, rraisemblablement. dans la chute de la pression artérielle analogue, bien que moins intense, à celle que provoque l'injection d'extrait de sangsues et des peptones. Si l'on admet la théorie d'Atmanasiu et Carvallo sur le méranisme de rette diminution de coagulabilité, les phénomènes s'expliqueraient bien. Rappelóns que, pour ces auteurs, les subslane's capables de produire une raso-dilatation abdoninale déterminent une diminution de la coagulabilite du sang, les leucocytes se trourent, par suite de rette raso-dilatation, en quantité inférieure à la normale dans le sang phériphérique; le tibrine-ferment, par eux excrété, se trouve aussi en quantité inférieure à la normale dans ce même sang.

L'examen du tracé de pression artérielle permet également de se rendre compte des varialions de lactivité respiratoire et cardiaque. Les grandes oscillations du tracé, dues aux mouvements respiratoires, diminuent considérablement d'amplitude pendant toute la période d'intoxication, puis remontent peu à peu à la normale, lorsque l'aclion du toxique se dissipe. Nous pouvons done dire que, sous l'influence de l'extrait, l'amplitude des mouvements respiratoires diminue, mais que leur rythme n'est sensiblement pas modifié. Même résultat en ce qui concerne les contractions cardiaques, traduites sur le tracé par les petites oscillations superposées aux grandes. Le rythme cardiaque n'étant pas altéré, il est évident que l'extrait n'agit en rien sur les centres modérateurs ou accélérateurs du cour, ni sur les ganglions intra-cardiaques. 
L'après-midi, le chien est totalement rétabli. Nous pouvons done conclure qu'une dose de $\ddot{j} \mathrm{c}^{3}$, pour un chien de poids moyen, suffit à déterminer des phénomènes d'intoxication passagère, surtout marquée par une chute brusque, mais momentance, de la pression artérielle. La cause de cette chute ne peut être attribuée à l'affaiblissement des contractions cardiaques; les deux phénomènes ne sont pas en rapport suffisamment direct.

Telle n'élait pas la conclusion d'IIutcursson qui, expérimentant avec l'extrait à la dose de $\ddot{a} c^{3}$, a noté une diminution assez forte de l'amplitude et du nombre des contractions cardiaques ; aussi, est-il tenté de rapporter à ces modifications du cocur la cause première de la chute de la pression. Nous sommes cependant d'accord avec cet auteur pour conclure qu'il n'y a pas de stimulation des centres cardiaques inhibiteurs, centraux et périphériques.

N'ayant pu observer d'altération du rỵthme cardiaque, il nous était inutile de reprendre l'expérience après la section des vagues. Cetle expérience, tentée par Hutcunson, lui a montré la même chute de pression et le même ralentissement du pouls. Ce résultat est assez difficile à interpréter; les vagues sectionnés, le pouls devrait s'accélérer; or, l'injection d'extrait le ralentit encore. L'Actée agirait-elle donc sur le ccur (poison nerveux ou musculaire)? L'expérience répond à cette hypothèse par la négative. Ou bien agirait-elle en augmentant la pression générale (vaso-constriction), et le ralentissement du rythme cardiaque ne serait-il qu'un phénomène consécutif et sous la dépendance du phénomène vasomoteur? Deuxième hypothèse également contredite par l'expérience.

Nous devons ajouter d'ailleurs qu'Hutcmisson semble bien admettre, comme nous, que le principal facteur de la diminution de pression artériclle est une vaso-dilatation (vraisemblablement de la circulation abdominale).

Exp. XII. - Même chien que ci-dessus, le lendemain de son rétablissement.

11 h. 3. - $15^{c^{3}}$ d'extrait, d'un coup, dans la saphène. Les phénomènes sont entièrement comparables, quant à la chute 

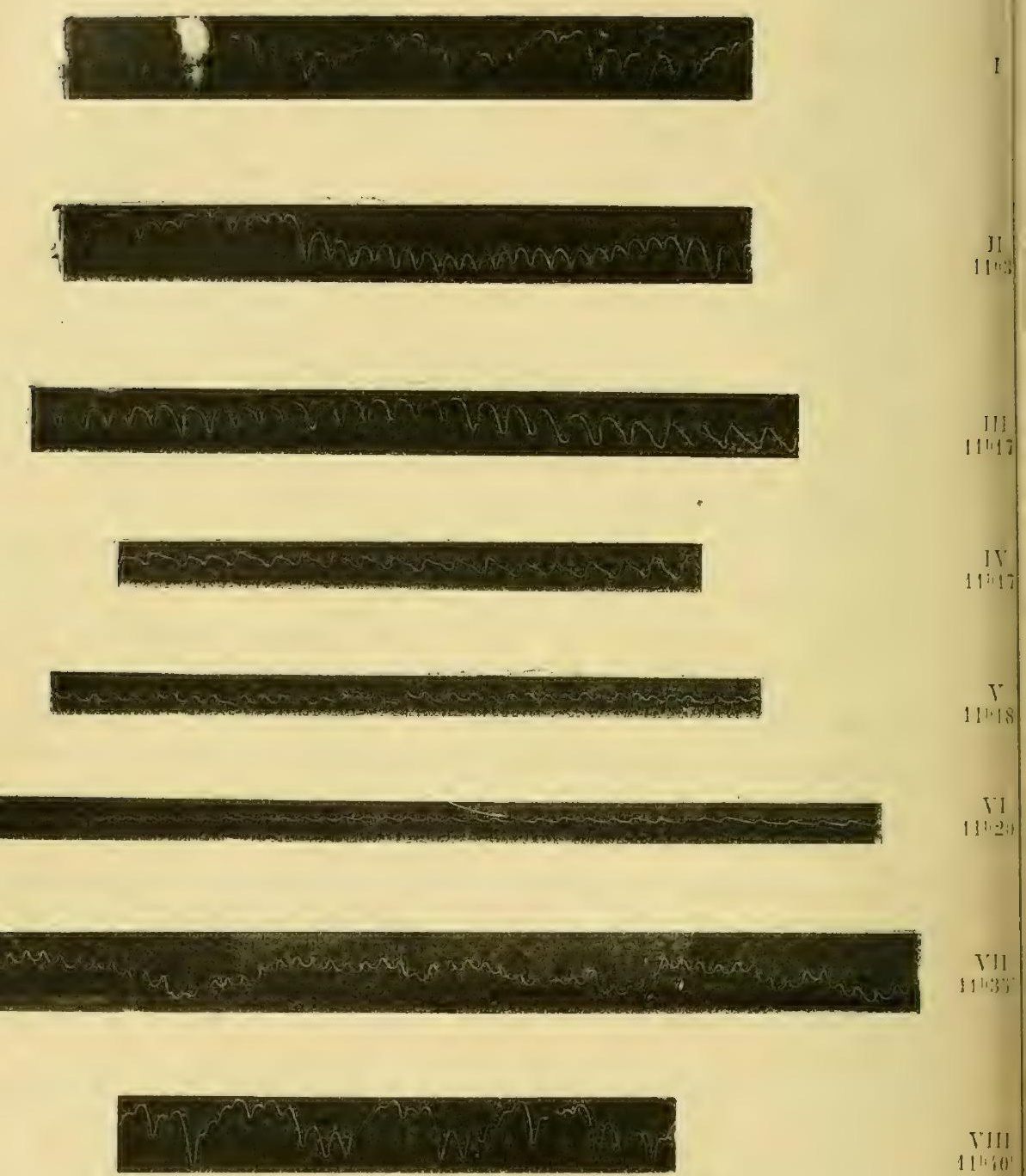

lig. 72. - Modifications de la prossion actirielle chez le chien, après injection (Expér. XII.) intra-veineuse d'extrail fluide $\left(15+15^{\circ 3}\right.$. $)$

(Manomètre double de Fr. Franck, pression carotidienne.)
Phot. directe, réauction au tiers) tracé normal

La vitesse du cylindrs enregistreur est plus forte que dans l'expérience précédente, ainsi que la pression normale. 
de la pression et aux modifications respiratores et cardiaques, il ceux de l'expérience ci-dessus fii l'ỉ tensité près, puisque la dose du toxique est triple (II)].

11 h. 17. - Lorsque la pression tend à revenir fa normale (III), nouvelle injection de $15^{3}$ dans la sapherte. La pression de $12 \mathrm{~cm}$ tombe immédiatement à 6 , puis à $3(\mathrm{IV}, \mathrm{V})$ la disparition, dans le tracé, des grandes oscillations indique la cessation jresflue complete des mouvements respiratoires (VI). L'amplitude des contractions cardiaques diminue aussi de la façon la plus notable (le cour cesse d'être perceptible au doigt), mais leur nombre ne varie point; la pression est alors égale à 0 , et tous les réflexes ont disparu (VI). L'animal semble en état de mort imminente.

11 h. 35. - La pression monte, l'amplitude des pulsations cardiaques s'accentue, ainsi que celle des mouvements respiratoires.

Après plusieurs romissements l'animal commence à se réveiller.

11 h. 40. - La pression est considérablement remontée $12 \mathrm{~cm}$.

L'après-midi l'animal est encore en vie, mais inerte, râlant et agité de petites convulsions généralisées. La mort survient i 5 heures.

Avec une dose aussi forte $\left(30^{\mathrm{c} 3}\right)$ la chute de la pression n'est plus seulement attribuable i des modifications raso-motrices, son facteur principal est évidemment l'affaiblissement de la contraction cardiaque. Il est à noter qu'alor's que les mouvements respiratoires sont presque totalement arrêtés, le cœur continue à battre; pendant toute celte période, où l'animal est éxidemment en état d'asphỵie, la pression artérielle devrait normalement s'élever. On sait, en effet, que dans l'asphyxie de cause mécanique, par suite de la vaso-dilatation périphripique et de la vaso-constriction abdominoviscérale, la pression augmente (Ludw el THRY).

Ici, au contraire, la pression se maintient à un chiffre extrêmement faible, et tombe même à 0 .

Ce qui amène à conclure que, sous l'influence de l'extrait, il y a vaso-dilatation abdomino-viscérale, et que les centres vaso-moteurs, en état de paralysie, sont incapables de répondre à l'excitation que détermine normalement le sang asphyxique. 
L'extrait d'Actée apparait donc comme un toxique paralysant des centres vaso-moteurs. On devrait alors s'attendre à le voir primitivement exciter ces centres et déterminer ainsi, tout à fait au début de son action, une augmentation de la pression. Nous n'avons pu constater le fait et, par là, ce toxique semble échapper à cette règle, presque générale, que tout toxique qui déprime un élément anatomique, commence tout d'abord par l'exciter.

Rem. - La dose toxique d'extrait dans l'injection intra-veineuse est donc de $30^{\mathrm{c} 3}$ pour un chien de 6 kilos, et encore la mort ne se produit-elle qu'au bout de 6 heures. La toxicité de cet extrait est donc bien inférieure au chiffre indiqué par Hưchinson (dose mortelle $10^{\mathrm{c} 3}$ pour un chien de 11 kilos).

\section{EXPÉRIENGES AVEC L'ACTÉINE}

La présence dans l'extrait fluide d'une quantité assez considérable d'alcool introduit forcément une cause de doute sur l'action propre des principes actifs de la plante. L'expérimentation avec l'actéine pure est d'un intérêt beaucoup plus considérable, tant au point de vue théorique qu'au point de vue pratique.

\section{$1^{\circ}$ Actéine pulvẻrisée.}

Grenouille.

Par voie stomacale. - Quelques goultes d'eau tenant en suspension de l'actéine en poudre, injectées ì la seringue dans l'œsophage d'une grenouille, ne déterminent aucun accident; mais il est à noter que, comme il est très difficile de faire pénétrer le liquide dans l'œsophage, la majeure partie peut être rejetée du pharynx par les mouvements de projection en avant de la langue.

Par absorption cutanée. - Une grenouille, placée dans une mince couche d'eau, tenant en suspension une forte quantité d'actéine, vit, sans inconvénient, pendant deux semaines, au contact de ce principe; son absorption par la peau est donc nulle. On sait, d'ailleurs, que la peau humide de la grenouille absorbe mal, même les substances dissoutes. 
L'absorption de l'actéine en suspension dans l'eau, par la peau sèche, du mème animal, semble également nulle.

Par voie lymphatique. - On peut, sans inconvénient, injecter dans le sac lymphatique dorsal 1 à $2^{c 3}$ d'eau stérilisíe, tenant en suspension de l'actéine pulvérisée.

L'expérimentation sur la grenouille n'est donc susceptible de nous fournir aucun résultat.

\section{Cobaye.}

Commençons par relater quelques essais négatifs que nous avons tentés, après nous être assuré des propriétés irritantes de l'actéine, sur lesquelles nous insisterons plus loin.

Sur la cornée. - La poudre d'actéine déposée sur le globe oculaire est éliminée peu à peu sans déterminer aucun phénomène appréciable, par le clignement des paupières.

Sur le derme. - La même poudre, déposée sur le dos, à la surface du derme, mise à nu par friction avec de l'ammoniaque, ne détermine aucune irritation appréciable, et ne retarde nullement la cicatrisation.

\section{Par voie hypodermique.}

Exp. XIII. - 5 5³ d'eau stérilisée, contenant en suspension 2 gr. 5 d'actéine en poudre, sont injectés sous la peau du flanc d'un cobaye de poids moyen. Liinjection est douloureuse; pendant les 24 hlh. qui suivent cette injection, hyperesthésie marquée de toute la région injectée. L'état général persiste à être bon; il ne se forme, au point d'inoculation, ni œdème ni collection purulente. L'actéine injectée détermine la formation de petits nodules indurés, qui augmentent d'abord de volume pendant les 1 ă jours qui suivent l'injection. Pendant cette période, le poids de l'animal diminue sensiblement (de $60 \mathrm{gr}$. environ). Au bout d'un mois, le nodule persiste; à l'autopsie, on retrouve, en outre, une masse d'actéine, entourée d'une zone de tissu fibreux de nouvelle formation.

Il est rraisemblable que l'actéine, introduite dans le tissu cellulaire sous-cutané, se borne ì y déterminer (par action chimiotactique?) l'afflux de leucocytes, et la formation d'une zone fibreuse 
d'induration, plus étendue que s'il s'agissait d'une substance inerte.

Administrée par cette voie, la substance est sans retentissement sur l'élat général (sauf une diminution temporaire de poids) due à la suppuration.

Par voie diyestive. - L'actéine pulvérisée, en suspension dans l'eau, détermine en $̋ ّ$ heures la mort d'un cobaye, de poids moyen, à la dose de $0 \mathrm{gr} .25$.

A la dose de 0 gr. $ّ 0$, la mort est un peu plus rapide (au bout de $1 \mathrm{~h}$. .); rien n'est modifié dans l'allure de l’intoxication, ni dans les lésions constatées à l'autopsie, par l'ingestion de doses plus fortes. Plus la dose est forte, plus les lésions du tube digestif sont intenses.

Les expériences donnent des résultats tellement concordants, que la relation d'une seule d'entre elles suffira.

Exp. XIV. - Cobaye mâle, vigøureux, neuf, $\mathrm{P}=655$ gr. R. 55. C. 140 . T. $39^{\circ}$.

10 h. 30. - Dans l'œsophage, par la sonde $0 \mathrm{gr}$. 25 d'actéine en suspension dans l'eau.

11 h. - R. 79. C. 147 . T. $39^{\circ}, 2$.

La respiration est accélérée comme le cœur, des expirations forcées (sortes de hoquets) répondent peut-être à des efforts nauséeux avortés. Vivacité très grande, réaction à la moindre excitation, motricité intacte.

11 h. $30 .-$ R. 60. C. 139 . T. $38^{\circ} 7$.

11 h. $45 .-$ R. 54. C. 138 . T. $38^{\circ}$.

La respiration se ralentit ainsi que le cœur, la température tombe, peu à peu cet état de collapsus s'accentue, les réflexes s'atténuent.

1 h. - L'animal entre dans le coma, avec hypothermie marquée $\left(30^{\circ}\right)$; à intervalles presque réguliers, surviennent des convulsions de courte durëe; rejet par les narines de mucosités spumeuses, respiration lente et faible, accompagnée d'un bruit de râle (V.tracés p. 317); mouvements d'expiration forcée se reproduisant à intervalles réguliers; l'inspiration ressemble plutôt à un hoquet; par instants, émission d'une faible quantité d'urine; des mouvements spontanés couvulsifs indiquent que la motricité reste intacte; sensibilité aux excitations fortes et douloureuses encore conservée. 
4 h. - Mort par arrêt de la respiration et du cocur.

Autopsie immédiate :

OEsophage. - Normal, n'a pas subi le contact de l'actéine, la sonde ayant été enfoncée jusqu'ì l'estomac.

Estomac. - Congestion légère à la surface externe; petites ecchymoses punctiformes disséminées, le contenu stomacal exhale une forte odeur d'actéine; forte et par larges plaques à la surface interne, surtout le long de la grande courbure.

Intestin. - Intestin grèle, de couleur hortensia, à surface interne présentant de larges plaques de congestion intense, distendu dans toute la longueur par un liquide sérosanguinolent, de consistance un peu muqueuse, exhalant une forte odeur d'actéine, renfermant une grande quantité de cellules épithéliales desquamées, des leucocytes et quelques hématies.

Gros intestin normal, le toxique n'a pas franchi le cxcum, et la sérosité accumulée dans le duodenum y a été retenue par un amas de fèces à ce niveau.

Foie. -Congestionné, piqueté rouge, intralobulaire; vésicule biliaire rempli de sérosité rosée.

Reins. - Piqueté rouge à la surface, surtout net à la coupe tangentielle (glomérulite); un sang noir s'écoule à la section.

Organes génitaux internes. - Congestionnés.

Les veines du mésentère et tous les vaisseaux abdominaux sont fortement congestionnés.

Poumons. - Asphyxiques, marbrés, ecchymotiques; par places de larges infarctus noirâtres, fournissant à la coupe du sang noir.

Cœur. - Ventrïc. g. en systole, vide, cœur dr. et or. g. remplis d'un sang noir, fluide.

Encéphale. - Congestion des méninges.

\section{Examen histologique du tube digestif (1).}

Le tube digestif, fixéaussitôt au sublimé aceitique, est l'objel d'un examen ultérieur, dont voici les résultats :

Pour l'estomac, il s'agit d'une gastrite superficielle aiguë desquamative. Les lésions consistent en la production, à la surface de la muqueuse, d'une mince couche peudo-membraneuse formée de cellules épithéliales desquamées et fragmentées, de noyaux ou de fragments de noyaux, de globules rouges du sang et de leucocytes agglomérés par une gangue de mucus.

(1) Cet examen a été pratiqué par le Dr Mént́trier, professeur agrẻgé à la Faculté, à la demande de M. IIeim, auquel il a bien voulu remettre la cette note. 
Au-dessous, la surface de la muqueuse est dépouillée de son épithélium cylindrique superficiel, au niveau desportions saillantes des plis de la muqueuse, tandis que, dans les dépressions, l'épithélium persiste sous la pseudo-membrane. Même au niveau des points desquamés les orifices glandulaires ont également conservé leur revêtement épithélial cylindrique. Plus profondément les glandes n'ont subi aucune altération, les épithéliums glandulaires sont partout conservés et ne présentent aucune modification ni de leur situation, ni de leur morphologie, ni de leurs réactions colorantes. Il n'y a aucun signe de prolifération cellulaire.

Le tissu conjonctif interglandulaire présente une légère infiltration leucocytique, surtout au voisinage de la surface, entre les orifices glan-. dulaires et plus particulièrement au niveau des points desquamés. Dans la profondeur, au niveau des culs-de-sac, la diapédèse est peu marquée, et il y a seulement un peu de congestion vasculaire; quelques capillaires dilatés sont remplis de globules.

Aucune altération des autres couches de l'estomac.

Dans l'intestin grêle, l'épithélium de revêtement est partout conservé et non altéré. Il est, au niveau des dépressions, entre les villosités, recouvert de mucus, avec quelques globules blancs dégénérés, et peu de globules rouges en amas irréguliers. Il y a une infiltration leucocytique assez notable et diffuse, dans le tissu conjonctif des villosités, et un peu de congestion sanguine. Pas de lésions des glandes, ni d'altérations des autres tuniques de l'intestin.

Ce sont en somme des lésions assez banales d'irritation superficielle récente, un peu plus marquées au niveau de l'estomac que de l'intestin.

\section{Chien.}

Par voiestomacale. - Le chien est beaucoup moins sensible que le cobaye à l’ingestion par voie stomacale d'actéine. Unchien, du poids moyen de 10 kilos, peut impunément ingérer de 1-2 gr. d'actéine pulvérulente, soit avec ses aliments, soiten suspension dans l'eau. Malgré la facilité avec laquelle cet animal romit, on ne constate qu'assez rarement des nausées. Les modifications qui surviennent (à la suite de l'ingestion d'une semblable dose unique), du côté des grandes fontions ou de l'état général sont inappréciables. L'ingestion de 5-6 grammes, d'un coup, d'actéine amène la mort, après aroir déterminé des phénomènes locaux de gastro-entérite, et des phénomènes généraux, qui reproduisent si bien ceux ohservés chez. le cobaye, que relater la marche de l'intoxication et les lésions 


\section{$-261$}

constatées à l'autopsie, ne conduirait qu'à des redites inutiles. Le chien se prète fort bien, en raison mème de son peu de sensibilité à l'artéine, à l'essai de polites doses suceessives journalieres. Cette expérience présente un intérét spécial, au point de vue des essais thérapeutiques à tenter chez l'homme.

\begin{tabular}{|c|c|c|c|c|}
\hline \multicolumn{5}{|c|}{$x p$. XV. — Chien neuf. } \\
\hline \multicolumn{2}{|c|}{ DATES } & POIDS & \multicolumn{2}{|c|}{ ACTÉINE (par sonde stomacale). } \\
\hline $1^{\text {er }}$ & Fév. & 8 , kilos & $1 \mathrm{gr}$. & $\begin{array}{l}\text { Rejet d'une petite quantité de mucus } \\
1 \text { heure après l'ingestion. }\end{array}$ \\
\hline 2 & ” & ) & $1 \mathrm{gr}$. & 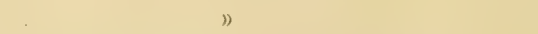 \\
\hline 3 & 》) & 》) & 0 & $\begin{array}{l}\text { Inappétence totale, constipation mar- } \\
\text { quée, état maladif. }\end{array}$ \\
\hline 4 & ) & $7 \mathrm{k} \cdot 940$ & $01 \mathrm{gr}$. & $\begin{array}{l}\text { État général mauvais, rejet des mucus, } \\
\text { bave abondante, visqueuse, spu- } \\
\text { meuse, s'écoulant spontanément de } \\
\text { la gueule; gémissements, anurie, } \\
\text { constipation et inappétence persis- } \\
\text { tantes. }\end{array}$ \\
\hline 5 & 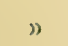 & 7 k. 920 & $0 \mathrm{gr}$. & Amélioration de l'état général. \\
\hline 6 & " & ” & $0 \mathrm{gr}$. & $\begin{array}{l}\text { Après expulsion de fèces sèches, } \\
\text { dures, exhalant l'odeur d'Actéine, } \\
\text { l'appétit reparait, l'état général } \\
\text { redevient normal. }\end{array}$ \\
\hline
\end{tabular}

7,8,9,10,11,12 Fév. 7 k. 9550 gr. Cessation de l'expérience. 13 Fév.
$1 \mathrm{gr}$. Rejet d'une faible quantité de mucus.

14 " $7 \mathrm{k} .930 \quad 0 \mathrm{gr}$. Constipation, inappétence.

L'expérience est continuée comme ci-dessus et donne un résultat identique.

Il n'y a donc aucune accoutumance du tube digestif à des doses assez fortes d'actéine.

En résumé, l'actéine en poudre même à doses fortes détermine aussi chez les chiens des phénomènes de gastro-entérite, d'une gravitémoyenne, à cause du peu de susceptibilité du tube digestif chez cet animal. 


\section{$2^{\circ}$ Actẻine en solution sodique.}

Les essais relatés ci-dessous ont été effectués avec la solution sodique, préparée comme il a été indiqué au Ch. III.

Rappelons que charque ${ }^{\text {c3 }} \mathrm{de}$ cette solution contient 0,02 d'actéine pure, et 0,03 de carbonate de soude.

\section{Cobaye.}

Par voie hypodermique.

Exp. XVI. - Cobaye femelle, neuve. Poids 5i: grammes. Recoit dans le flanc $3^{3^{c 3}}$ de la solution; aucun phénomène appréciable. Le lendemain état normal, mais, au bout de 48 heures, un abcès se forme au point d'inoculation, et se vide spontanément au bout de trois jours. Il en résulte une large surface bourgeonnante, dont la cicatrisation se fait avec une fort grande lenteur. Le pus de l'abcès examiné bactériologiquement est amicrobien.

La solution sodique d'actéine est donc phlogogène; mais cette propriété appartient bien plutot au sel de soude qu'elle renferme en fortes proportions qu'à l'actéine elle-même qui, nous l'avons vu précédemment, est impuissante à déterminer la supuration. D'ailleurs l'importance pralique de ce fait est insignitiante. Cette propriété phlogogène empèche de renouveler l'expérience avec des doses plus fortes, car tout retentissement sur l'état général, se produisant quelques heures après l'inoculation, pourrait ìtre mis sur le compte de la suppuration commençante.

Par voie péritonéale.

Exp. XVII. - L'injection aseptique, dans le péritoine d'un cobaye, de poids moyen, de šc3 de solution détermine, au bout de 10 heures environ, de l'inertie, de la parésie du train postérieur surtout du côté inoculé, et la mort au bout de 15 à 16 heures.

A l'autopsie imédiate, on trouve:

OEdème gélatineux jaunâtre, violacé par places, de tout le tissu cellulaire sous-cutané de la région abdominale, surtout autour du point d'inoculation; péritoine rempli d'une sérosité roussâtre que l'examen bactériologique montre privée de tout germe.

Tube digestif.-Normal, sauf au niveau du cæcum et du cólon dont la surface présente une congestion intense.

Foie. - Congestionné; vésicule biliaire vide. 
Rein. - Organes génitaux internes congestionnés.

Poumons. - Asphyxiques, d'un rouge brique avec nombreuses petites ecchymoses noires.

Cœur.-Oreillettes encore contractiles, gorgées de sang noir fluide ainsi que le ventricule droit. Le cocur s'est arrêté en diastole.

Encéphale. - Congestionné.

La mort est, dans cette expérience, très certainement déterminée par l'irritation aseptique du péritoine.

Il est à remarquer que le contact de la solution d'actéine avec la surface externe de l'intestin détermine (arec moins d'intensité), aussi bien que le contact de l'actéine en poudre avec la surface externe, une congestion marquée de l'organe.

Il est sans grand intérêt d'étudier avec cette solution l'intoxication par vie stomacale. Les cssiais avec lactéine pulvérulente nous ont suffisamment renseigné à cet égard. Le seul avantage de la solution sodique pour l'expérimentation physiologique est la possibilité de porter directement le toxique dans la circulation, et d'étudier les modifications physiologiques attribuables à l'acténe, en dehors de toute action locale sur le tube digestif.

\section{Lapin.}

Influence sur la contraction cardiaque. - Il est particulièrement intéressant de rechercher si la solution d'actéine est susceptible, comme l'extrait fluide, d’imprimer quelques modifications à la contraction cardiaque.

Exp. XVIII. - Une injection de $3^{\mathrm{e} 3}$ de solution dans le péritoine du lapin ne détermine que des modifications insignifiantes dans l'amplitude des contractions cardiaques.

Il ny a aucun changement, pendant l'heure qui suit l'injection, dans les rythmes respiratoires ou cardiaques. Les tracés ci-contre (recucillis arec le cardiographe Bardier, indiquant a la fois les mourements respiratoires et cardiaques, sont démonstratifs à cet égard. 


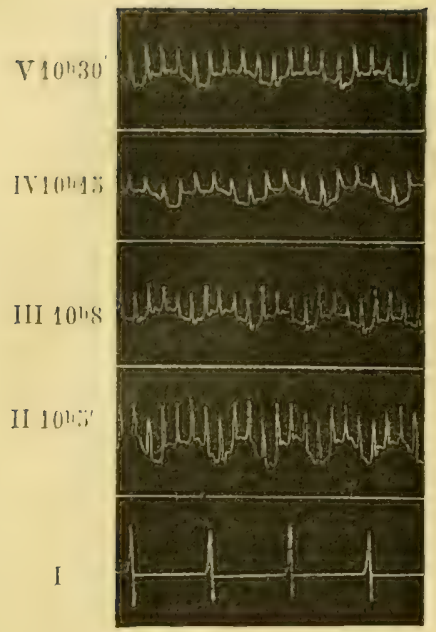

Nic. 73. - Modifications de la contraction (ardiaque chez le Lapin, sous l'influence de la solution sodique d'actéine. (Voie péritonéale). (Lispér. XVIII) (Cardiographe Bardier).

(Phot. directe sans réduct.)

Les tracés indiquent à la fois le cœur et la respiration.

I tracé du métronome, battant la seconde. II tracé normal au moment de l'injection.

Chien.

Par voie stomacale. - Une forte dose de solution (30 et même $\check{30} 0^{r 3}$ d'un coup) portée directement dans l'estomac par la sonde, ne détermine chez le chicn aucun accident fâchrux. Tout se borne à un rejet plus ou moins alondant de mucus, selon la sensibilité plus ou moins grande de la muqueuse, chez l'animal en expérience.

Par voie intra-veineuse.

Influence sur la pression artérielle. - Les modifications de la pression, provoquées par l'injection d'extrait fluide, se reproduisentelles à la suite de l'injection d'actéine? L'expérience répond par la négative.

Exp. XIX. - Chien neuf. Poids 5 kilos. Pression carotidienne enregistrée par le manomètre double de Francois Franck.

11 h. 3.- $-15^{\mathrm{c} 3}$ de solution dans la saphène, chute immédiate de la pression, mais au bout de quelques secondes, elle est revenue à la normale.

$11 \mathrm{~h} .15 .-$ Nouvelle injection de $15^{3}$ dans la saphène, même chute de la pression, plus accusée, se prolongeant pendant quelques secondes de plus. Après cette très courte période pendant laquelle l'amplitude des contractions cardiaques est diminuée, la pression revient entièrement normale. 


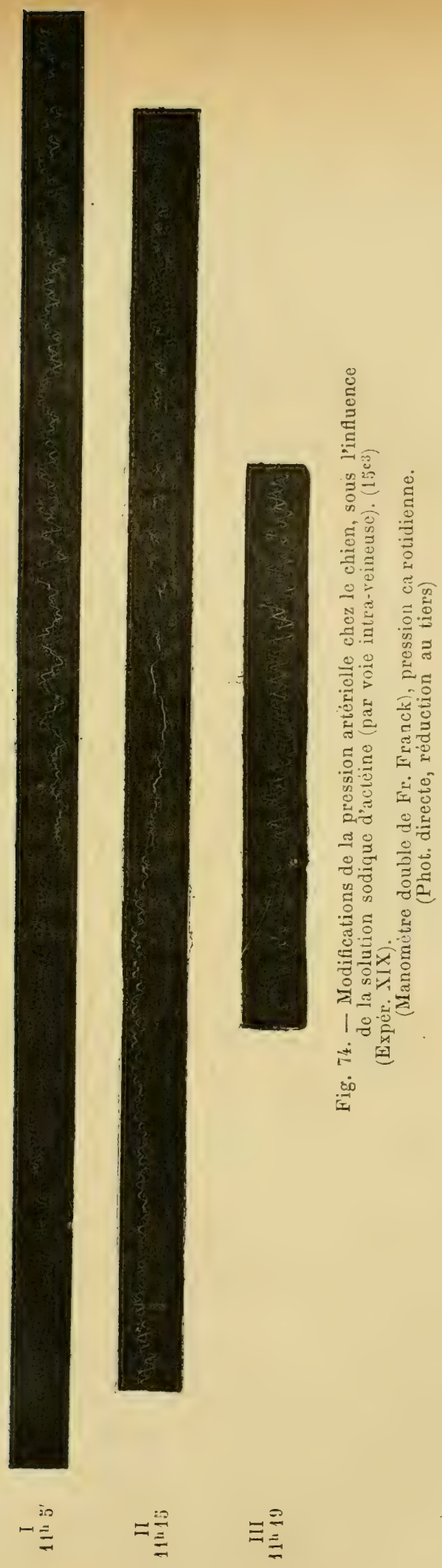


La diminution de force de la contraction cardiaque suffit ì expliquer cette chute si monentance de la pression, et l'action (toul aussi courte) dépressive de l'injection sur le cœur est entièrement attribuable au carbonate de soude injecté. Semblable baisse s'olserverait d'ailleurs à la suite de l'injection de n'importe quelle substance faiblement irritante pour l'endocarde. Noussmmes donc amenés à conclure que ce n'esl pas à l'actéine que l'extrait fluide doit son action sur la circulation et la respiration.

\section{L'intoxication par l'Actra racemosa chez l'homme.}

Nous relatons plus loin les phénomènes d'intolérance gastrointestinale (avec retentissement plus ou moins marqué sur la respiration et la circulation), consécutifs à l'ingestion de doses un peu fortes d'actéine en poudre.

Il existe quelques relations de cas d'intoxication survenus chez l'homme à la suite de l'ingestion, par mégarde, d'une dose trop forte d'extrait fluide.

La plus complète, due à Brainaro, et qui a le mérite d'être relative à un cas personnel, peut avantageusement être résumée ici.

"Le 26 décembre, dit cet auteur, je pris 3 grammes d'extrait de Cimifuga, et $1 / 2$ heure après, environ, j'éprouvais une sensation de lourdeur, de plénitude à la tête; la face était rouge, une impression de chaleur s'élendait à tout le corps, avec des vertiges, que la station debout exagérait encore. Un sentait aussi une insupportable douleur à la base de l'épine dorsale.

"Après une heure, tous ces symptômes s'accentuèrent. Il y avait de la rougeur des yeux, mais les pupilles étaient normales, de mème que la température du corps. Le pouls marquait 100, était plein, et annonçait une augmentation dans la tension artérielle.

"A aucun moment ralentissement du pouls, ni faiblesse cardiaque.

"Le mal de tête devint excessivement violent, et la moelle était évidemment très stimulée. Les muscles du dos, des bras, des jambes étaient dur's et tremblants. Deux heures plus tard ces symp- 
Lomes continuèrent, s'aggravèrent et les nausées apparurent. Il y eut péristaltisme exagéré, mais non purgation. Quatre heures après avoir pris le poison je bus un peu d'eat chaude, el je vomis durant les $ّ$ heures qui suivirent.

" Les symptomes continuèrent sans amélioration jusqu'ù 8 heures. Le mal de tête était si sévère que ma femme fut forcée de m'anesthésier avec du chloroforme.

"Forte douleur du dos et grande agitation; huit heures après que j'eus pris le poison, le sommeil vint, et je m’éveillai, quelque temps après, avec un priapisme marqué.

"Les effets sur l'épine dorsale et les nerfs se firent sentir encore un peu pendant deux jours. La sécrétion bronchique étail considérablement augmentée.

Il n'y a pas eu d'augmentation dans la quantité d'urine émise, ni de modifications dins la sécrétion de la peau, durant toute la période de paroxysme."

Ce chapitre étant essentiellement documentaire, nous ferons plus loin les réflexions que nous suggèrent les essais physiologiques, ci-dessus mentionnés, relatives à l'action de l'Actée sur les divers systèmes organiques.

Bibl. 1887. - Hutchinson (R.). The physiological action of CimiciFUGa RACEMosa (Thérapeut. Gaz., vol. XI. sér. 3, III, pp. 731-33).

1893. - Brainard (I. N.) Physiological action of Cimicifuga Racemosa (Thérapeut. Gaz., vol. XVII, sér. 3 vol. IX, p. 369). 


\section{CHAPITRE VI}

\section{L'ACT AEA RAGEMOSA GOMME AGENT THÉRAPEUTIQUE}

Faisons pour l'instant abstraction des résultats fournis par l'expérimentation, relativement aux modifications physiologiques que l'Actée est susceptible d'imprimer aux diverses fonctions, et cherchons à vérifier, par la simple observation au lit du malade, les affirmations des auteurs sur l'efficacité de l'Actée dans diverses affections.

Ces affirmations une fois contrôlées, nous pourrons, en comparant les résultats cliniques aux données physiologiques, chercher à dégager le mode d'action de l'Actée sur les divers systèmes organiques; ses indications thérapeutiques, dans les nombreuses affections contre lesquelles on l'a préconisée, seront par là même singulièrement élucidées.

Nous n'avons pu expérimenter ses effets que dans un nombre de cas relativement restreint. Nous donnons donc ci-dessous de simples documents préliminaires, et non une étude thérapeutique d'ensemble de ce médicament.

Nous grouperons nos observations, autant que possible,par affection (1).

Comme l'Actée a été surtout vantée dans certains états chroniques, qui relèvent plus ou moins d'un vice général de la nutrition, beaucoup de nos malades présentaient à la fois plusieurs manifeslations pathologiques. C'est la manifestation prédominante qui nous a servi à établir un classement artificiel dans les observations.

(1) M. Bunti qui suivait, en même temps que nous, le service hospitalier de M. le Dr Dalché, a hien voulu nous aider à recueillir certaines de ces observations. Nous tenons â lui en adresser ici tous nos remerciements. 
Nous ferons préceder chaque groupe d'observations personnelles du résumé de l'opinion d'auteurs, relativement à son efficacité contre l'affection considérẻe.

De la sorte, il sera facile de se rendre brièvement compte de la valeur que l'on peul attribuer aux affirmations précédemment émises sur la valeur thérapeutique dı médicament.

Nota. - Pour abréger, nous n'indiquons pas toujours dans les observations les doses journalières d'actéine: ce sont celles indiquées au ch. Posologie.

Rem. - Dans tous les tableaux relatifs aux courbes d'urine, le terme résine est synonyme d'actéine.

\section{DANS LA BRONCHITE}

L'Actée est souvent prescrite dans la bronchite, comme calmant de la toux, et comme expectorant. Chapmas la déclare, à ce point de vue, médicament de valeur. Elle donnerait beaucoup de succès dans les cas " d'influenza, de catarrhe accompagné de migraine, de raideur des muscles, de douleur et de courbatures " (SrDney Rrveri), autrement dite de bronchite grippale. Sumpox vante ses bons effets dans les affections bronchiques des vieillards. BRtrToN la croit appelée à rendre de réels services dans le traitement de la bronchite, tant aiguë que chronique.

\section{A. - Bronchite chronique.}

Ons. I. - (Traitement par l'extrait fluide.)

La femme G... âgée de 72 ans, entre à l'hôpital le 8 janvier 1897.

ANTÉCED. HÉRÉD. - Parents morts vieux.

ANTÉCÉD. PERSON. - Règles à 13 ans, régulières, non douloureuses; 3 grossesses normales. Ménopause à 46 ans. En 1876, fluxion de poitrine, soignée à Beaujon, et accompagnée d'une perte vaginale de sang rouge. Pas de maladies infectieuses, pas de rhumatisme aigu, pas de fièvre typhoïde.

Histoire DE LA MALAdIE. - Depuis 1887, respiration courte, difficulté à monter, à marcher vite. Peu à peu; oppression presque continue s'améliorant par le repos, augmentant pendant le mouvement, et après les repas. La nuit est plutôt bonne : il ne semble pas y avoir de véritables crises d'asthme. La malade tousse énormément. Crachats glaireux. Symptòmes de courbature dans les épaules et entre les omoplates. 
ETAT ACTUEL :

Corur. - Normal.

Rein. - Pas d'albumine.

Systeme nerveux. - Normal.

Poumons. - Diminution du murmure vésiculaire. Râles sibilants et ronflants, ayant leur maximum aux bases, où s'entendent déjà quelques râles bulleux assez gros. Dyspnée : 34 respurations par minute.

Appareil digestif. - Bon appétit, mais digestion très difficile : la malade ressent de la pesanteur d'estomac, des bouffées de chaleur, un peu de vertige, de lassitude. Constipation opiniâtre. Gaz abondants.

Organes génitıux. - Normaux.

Rate. - Non perceptible.

Diagnostic. - Bronchite chronique.

Traitement :

9 janvier. - Badigeonnages iodés, sinapismes, extrait fluide $\mathrm{XX}$ gouttes.

16 janvier. - La malade trouve que la potion l'a fatiguée (du reste elle supporte mal les médicaments). Néanmoins la respiration est plus facile. Ext. fluide XXX gouttes.

11 janvier. - Ext. fluide XXXX gouttes.

13 janvier. - Beaucoup de mieux, expectoration facile. Disparition de l'oppression et des étouffements. Une selle, pas de crise diarrhéique.

14 janvier. - Pas d'extrait.

15 janvier. - Etat bon, une selle. Ext. fluide XX gouttes.

16 janvier. - Sortie de l'łıopital.

Fem. - La régularité des fonctions intestinales semble se manifester très nettement. La dyspnée a rapidement cédé.

Est-ce sous l'influence du médicament? Il serait aventuré de conclure, vu la brièveté du traitement.

\section{OBs. II. - (Traitement par l'extrait fluide.)}

La femme R..., âgée de 78 ans, entre à l'hôpital, le 5 janvier 1897 , pour des accès de dyspnée et de suffocation.

ANTÉCÉD. HÉRÉD. - Pas de renselgnements.

ANTÉCÉD. PERSON. - Plusieurs accès de dyspnée paroxystique l'an dernier.

État ACTueL. - Radiales flexueuses et athéromateuses. Nodosités arthritiques. Varices des membres inférieurs. Pas de fièvre. Langue bonne. Constipation.

Ceur. - Normal.

Poumons. - Percussion et auscultation donnent : emphysème, poussée légère de bronchite. Expectoration trìs peu abondante. 
Urines. - Légèrement albumineuses. Pas de sucre.

Diagnostic. - Bronchite chronique. Emphysème. Albuminurie, Herpétısme.

Traitement :

6 janvier. -10 Scammonée........................... a a 0 gr. 50.

2。 Extrait fluide.............. $\mathrm{XX}$ gouttes.

Eau sucrée.............. 60 gr.

8 janvier. - Diminution notable de l'oppression et de la toux. Bienêtre complet.

10 janvier. - Extrait fluide XXX gouttes. Pas de douleur d'estomac, pas de diarrhée. Ni étouflement, ni crachat.

11 janvier. - Extrait fluide XL gouttes.

12 janvier. - La malade quitte l'hôpital.

Rem. - La respiration semble plus facile après le traitement, la toux a également diminué.

OBS. III. - (Traitement par l'extrait fluide.)

Jean-Baptiste G... âgé de 73 ans, entre le 8 janvier 1897 à l'hòpital.

ANTÉCÉD. HÉRÉD. - Père et mère morts très vieux. 3 sœurs mortes d'hydropisie? de diabète? d'hépatite?

ANTÉCÉD. PERSON. - Érysipèle.

HISTOIRE DE LA MALADIE :

Depuis 10 ans, gêne de la respiration, sans véritables attaques d'asthme. Oppression quand l'expectoration est difficile. Depuis quelques mois, augmentation de la dyspnée. Pas de palpitations.

E'TAT ACTUEL :

Cœur. - Bruits un peu assourdis et lointains, 2c bruit seul un peu éclatant. Temporales non sinueuses. Pouls bien frappé.

Poumons. - Emphysème; râles sibilants.

Un:ines. - Rares, miction pénible. Pas de signes de brightisme, pas de sucre, pas d'albumine.

Appétit moyen. - Constipation.

Ongles épaissis. - Nodosités arthritiques, double hernie.

Diagiostic. - Bronchite chronique. Empliyseme. Surcharge graisseuse du cœur.

Traitenent :

15 janvier. - Urée 8 gr. 9 par litre. Extrait fluide XXX gouttes.

17 janvier. - Pas de doul. d'estomac. Légère céphalée. Diminution de la toux. Respiration pénible. Urine $1500^{\mathrm{cm} 3}$. Urée $15 \mathrm{gr} .3$ par litre. Extrait fluide XXX gouttes.

18 janvier. - Moins d'étouflements. Urines $1150 \mathrm{~cm} 3$. Même traitement. 
20 janvier. - Respiration plus facile. Moins de constipation, 1 selle. Mème traitement. Urines $1500^{\mathrm{c} 3}$.

22 janvier. - Etat général meilleur. Urines $1750^{\mathrm{c} 3}$.

24 janvier. - Mieux. Néanmoins quelques coliques. Expectoration assez ahondante. Urines $1750^{\mathrm{c} 3}$. Mème traitement.

25 janvier. - Urines $2000^{\text {c3 }}$. Même état, même traitement.

27 janvier. - Augmentation de la toux et de l'expectoration. Un peu de constipation. Urines 2 litres.

29 janvier. - Après sa potion, légère douleur d'estomac. Urines $1750^{\mathrm{c} 3}$.

31 janvier. - Pas d'extrait fluide. Toux fréquente. Pas de douleur d'estomac. Urines $2000^{\mathbf{e} 3}$.

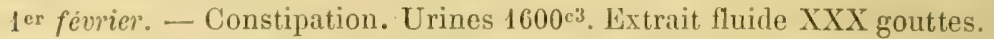

3 février. - Poitrine toujours un peu embarrassée. Urine $1300^{\mathrm{c} 3}$. Le malade demande à sortir.

OBS. III

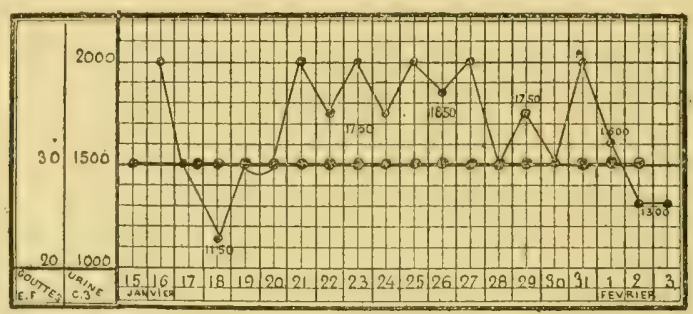

Rem. - Diminution de la constipation. Augmentation de la diurèse. Dans la première semaine du traitement, pendant laquelle le dosage de l'urée a été fait, on constate une augmentation du taux de l'urée.

OBs. IV. - Traitement par la poudre d'actéine.)

Charles WV..., âgé de 71 ans, entre à l'hôpital, le 24 octobre, pour une poussée de bronchite aiguë.

ETAT actuel :

Poumons. - Murmure respiratoire très affaibli, râles sibilants et ronflants disséminés dans toute la poitrine. Expectoration très abondante, aqueuse et spumeuse.

Cœur. - Normal.

Urines. - Pas d'allsumine, uro-hématine. Langue blanchâtre.

Diagnostic. - Artério-sclérose. Emphysème, bronchite chronique.

'T'RATTEMENT' :

25 octobre. - Actéine 1 cachet.

26 octobre. - Bonne nuit, n'a pas toussé, sauf une quinte le matin. 
Expectoration aussi abondante. Pas de maux d'estomac. Se trouve mieux. Actéine 1 cachet.

28 octobre. - Douleur gastrique, angoisse.

29 octobre. - Actéine 1 cachet.

30 octobre. - Devant l'intolérance gastro-intestinale pour le médicament, on doit interrompre le traitement.

OBS. V. - (Traitement: $1^{\circ}$ par la solution d'actéine; $2^{\circ}$ par l'extrait fluide.

Jean R..., polyscléreux, d'un aspect assez vigoureux, entre le 24 décembre à l'hòpital parce qu'il étouffe. Cet étoulfement est en rapport avec une bronchite chronique.

ÉTAT ACTUEL :

Poumon. - Sonorité exagérée. Signes d'emphysème et de bronchite chronique, gros râles disséminés.

Cœur. - Souffle systolique mitral.

Urines. - Claires, ni sucre, ni albumine.

Constipation habituelle.

Diagnostig. - Bronchite chronique.

Traitenent :

$1^{\circ}$ Par la solution d'actéine.

25 décembre. - Actéine 2 centigrammes.

27 decembre. - Expectoration aussi abondante, muco-purulente. Se sent plutôt mieux. Pas de maux d'estomac. Actéine 3 centigrammes.

28 decembre. - Même état, pas de douleurs d'estomac. Actéine 4 centigrammes.

30 décembre. - Va facilement à la selle : même un peu de diarrhée. Le malade étouffe un peu moins, mais crache autant. Actéine š centigrammes.

31 decernbre. - On suspend le traitement.

()BS. Y. 1 .

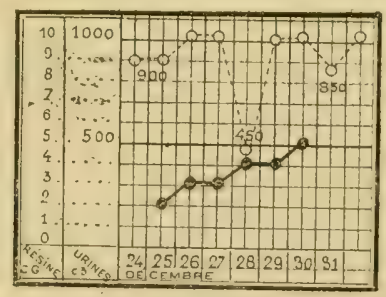




\section{Par l'extrait fluide.}

3 janvier 1897. - Même état. Le malade trouve qu'il tousse plutôt moins. Lxtrait fluide, $\mathrm{X}$ gouttes.

4 janvier. - Extrait fluide, $\mathrm{X}$ gouttes.

6 janvier. - Le malade n'étouffe presque pas. $\Lambda$ plutôt moins craché, semble mieux : va librement à la selle. Extrait fluide, XX gouttes.

8 janvier. - Etouffe plutot moins, mais souffre beaucoup au moment de la miction. Expectoration peu abondante. Extrait fluide, XXX gouttes.

10 janvier. - Avant le traitement était plutôt constipé, maintenant va 2 ou 3 fois à la selle par 24 heures. Extrait fluide, XXX gouttes.

11 janvier. - Extrait fluide, XL gouttes.

13 janvier. - Soufre moins. Suppression de la potion. Du 15 au 18 pneumonie et broncho-pneumonie qui emportent le malade.

OBS. Y. 2.

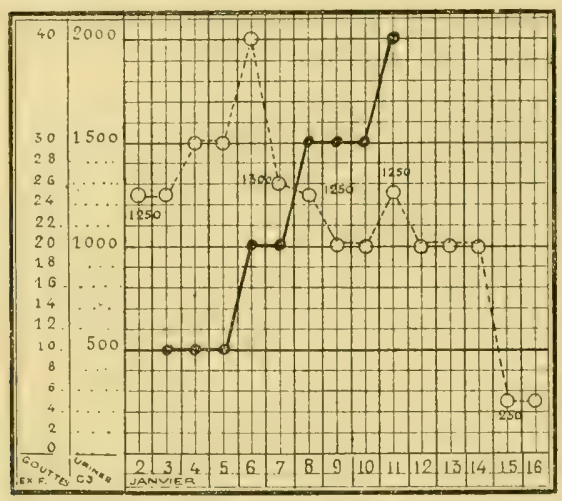

Rem. - La quantité d'urine ne semble pas proportionnelle à la quantité d'Actée administrée, ce qui s'expliquerait bien, si on admet que le médicament n'agit sur le rein que par voie réllexe. Chaque période de polyurie est suivie d'une phase d'hypo-urie.

La chute des urines correspond à la suppression du traitement.

(Il est vrai que le malade est un prostatique, avec cystite, ce qui rend un peu difficile l'appréciation juste de la quantité d'urine.)

Liextrail fluide semble déterminer une diurese plus abondante rue l'actéine alors que son administration n'introduit dans l'organisme qu'une plus faible dose d'actéine que celle qui était précédemment administrée en nature), à moins qu'il y ait une accumblation de l'actéine antérieurement administrée. 
V. aussi Observations VI, VIII, IX, X, : Bronchite chronique.

\section{B. - Bronchite aiguë}

OBS. VI. - (Traitement par l'extrait fluide.)

La femme B... entre à l'hôpital le 21 janvier 1897.

ANTÉGÉD. HÉrÉD. - Père bien portant, mère épileptique, morte à 36 ans, frères et soeurs bien portants.

ANTÉCÉD. PERson. - Premières règles à 16 ans, régulières, peu abondantes, non douloureuses. Pas d'enfant ni de grossesse. A 18 ans, choléra?... A 22 ans, bronchite.

HistoIRE DE LA MALADIE :

Le 8 janvier, la malade a un refroidissement, suivi d'une mauvaise nuit, sans sommeil et avec malaise. Le lendemain, oppression qui ne cesse plus, et pour laquelle elle entre à l'hòpital.

ETAT ACTUEL :

Facies normal, un peu pâle.

Cour. - Bruits forts et bien frappés. Pas de palpitations. Pouls un peu faible. - Artères souples.

Poumons. - Râles de bronchite diśséminés dans toute la poitrine, avec prédominance aux bases.

Thorax bombé d'emphysémateux.

Rein. - Normal, ni sucre, ni albumine.

Appétit médiocre. - Pas de constipation. Varices. Légères nodosités arthritiques.

Diagnostic. - Poussée de bronchite aiguë greffée sur bronchite chronique.

Traitement. - Extrait fluide, XX gouttes.

22 janvier. - Malade un peu mieux, léger tiraillement d'estomac.

23 janvier. - Journée assez bonne, mais étouffement la nuit. Céphalée. Extrait fluide, XX gouttes.

25 janvier. - Oppression, expectoration plus facile. Extrait fluide, XXX gouttes.

27 janvier. - Mieux : expectoration diminue. Pas de maux d'estomac, 1 selle.

29 janvier. - La malade se plaint de l'estomac et de la tête. Pas de diarrhée, mais après sa potion 3 selles. Suppression de l'Actée.

31 janvier. - Nême état.

$1^{\mathrm{er}}$ février. - Amélioration.

"sevvier. - Depuis trois jours, maux de tête au réveil, mais diminution de l'oppression.

8 février. - Amélioration sensible; la malade quitte l'ho̊pital le 9 février. 


\section{OBS. VII. - (Traitement par la pouđre d'actéine.)}

Le nommé D..., infirmier, entre à l'hôpital le 30 mai 96.

ANTÉCÉD. HÉRÉD. - Fièvre typhoïde à 20 ans. Variole dans l'enfance. Rhumatismes vers 19 ans; à 22 ans fièvre intermittente suivie de dysenterie.

ANTÉCÉD. PERSON. - Père mort à 52 ans de congestion pulmonaire; mère; frères et sœurs bien portants.

ÉtAт ACTUEL. - Depuis une dizaine de jours, toux, courbature, fièvre, pas d'expectoration, pas de râles dans la poitrine.

Diagnostic. - Bronchite grippale chez un malade, qui a un sommet avec une expiration rude et prolongée.

Traitement .

30 mai. - Actéine, 3 pilules.

$31 \gg \quad 4$ pilules.

I $^{\text {er }}$ juin. - Tousse et crache beaucoup moins, pas de râles dans la poitrine.

2 juin. - Tousse moins. Les pilules sont remplacées par 2 cachets de 15 centigrammes, 1 le matin et 1 le soir.

3 juin. - Même traitement.

4 juin. - Le malade va bien.

\section{OBS. VIII. - (Traitement par la solution d'actéine.)}

La veuve M... entre à l'hôpital, le 18 janvier 1896, pour bronchite et fièvre.

ANTÉCÉD. HÉRÉD. - Pas de renseignement.

ANTÉc. PERson. - Depuis quelque temps oppression, bronchites répétées, amaigrissement, migraines.

État actuel. - Nodosités arthritiques, maigreur, pas d'appétit, constipation.

Poumons. - Sonorité dans tout: le poumon. Murmure respiratoire étouffé, râles sibilants et ronflants disséminés.

Cæur. - Normal.

Urines. - Pas d'allumine.

Diagnostic. - Arthritisme, emphysème, poussée de bronchite aiguë. Traitement :

21 janvier. - Actéine, 2 centigrammes.

23 janvier. - La malade a beaucoup toussé le 22, mais la nuit du 22 au 23 a été bonne. Respiration plus facile. Urines, $400^{\mathrm{c} 3}$; actéine, 3 centigrammes. litre.

24 janvier. - Urines, 250c3. Pas d'albumine. Urée : 39 centig. 7 par 
25 janvier. - La malade tousse et crache autant. La température a baissé. Constipation. - Actéine, 3 centigrammes.

27 janvier. - Expectoration aussi abondante mais plus facile; actéine, 4 centigrammes. Petite crise polyurique : L'urine, de $: 50^{\mathrm{e} 3}$, est passée à $55^{\circ}{ }^{\mathrm{c} 3}$.

28 janvier. - La malade tousse moins, respire mieux. Urines, $430^{\mathrm{c} 3}$; actéine, 4 centigrammes.

30 janvier. - Toux et crachats moins fréquents. Urines, $750^{\mathrm{c} 3}$.

31 janvier. - La malade se sent mieux. Urines, $950^{\mathrm{c}^{3}}$. On continue toujours la potion.

3 février. - Amélioration sensible. Urines, $750^{\circ 3}$.

4 févier. - La malade quitte l'hôpital.

OBS. VHII.

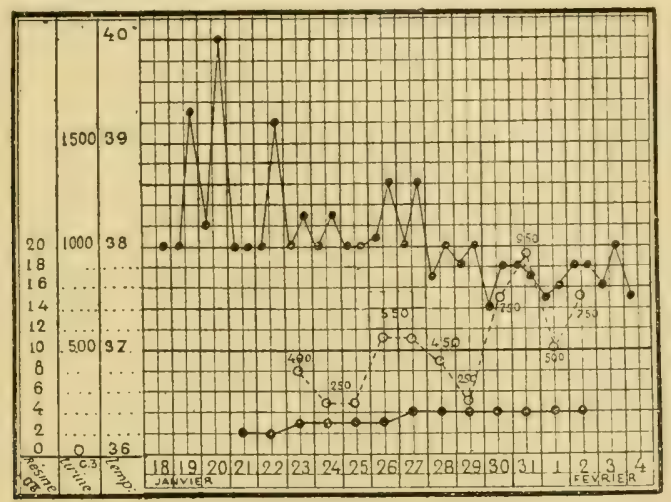

Rem. - La solution d'actéine ne semble pas avoir eu sur la régularité des selles la même action que l'extrait fluide.

V. aussi Observ. X. Bronchite aiguë.

\section{DANS L'EMPHYSÈME}

OBs. IX. - Traitement par l'extrait fluide.

Le nommé D... entre le 16 janvier 1897 pour la septième fois a l'hôpital. Il est depuis de longues années atteint de bronchite aux saisons froides; mais, depuis sept ou huit ans, il est plus souvent et plus longtemps atteint.

ÉtAT ACTUEL :

Poumons. - Submatité en arrière aux deux sommets. Râles sibilants disséminés partout. Crachats peu abondants, oppression. 
Ceur. - Normal.

Urine. - Ni albumine, ni sucre. Urée $23^{\mathrm{c}}$ par litre;

Pas de constipation habituelle.

Diagnostic. - Emphysème. Artério-sclérose. Bronchite chronique.

Traitement :

17 janvier. - Extrait fluide XXX gouttes.

18 janvier. - Pas de douleur d'estomac.

20 janvier. - Mieux. Respiration plus facile. Extrait fluide XXX gouttes.

22 janvier. - Tousse et crache. Légères douleurs d'estomac.

23 janvier. - Suppression de l'Actée.

24 janvier. - Maux d'estomac. Pas d'appétit. Urine 1250³,

25 janvier. - Souffre de l'estomac. Ext. fluide XXX gouttes.

27 janvier. - Maux d'estomac. Suppression de l'actée. Lait. Laudanum.

29 janvier. - Même état.

1er février. - Teinture de kola et de coca.

3 féviier. - L'estomac s'est calmé.

ö février. - Étouffe toujours et souffre de nouveau de l'estomac. Le malade se lève, mais no peut marcher.

7 février. - Même état.

8 février. - Sirop de morphine $30 \mathrm{gr}$.

10 février. - Mieux très sensible.

12 février. - Le mieux s'accentue.

Du 19 aи 20. - L'amélioration se continue.

21 février. - Le malade quitte l'hôpital.

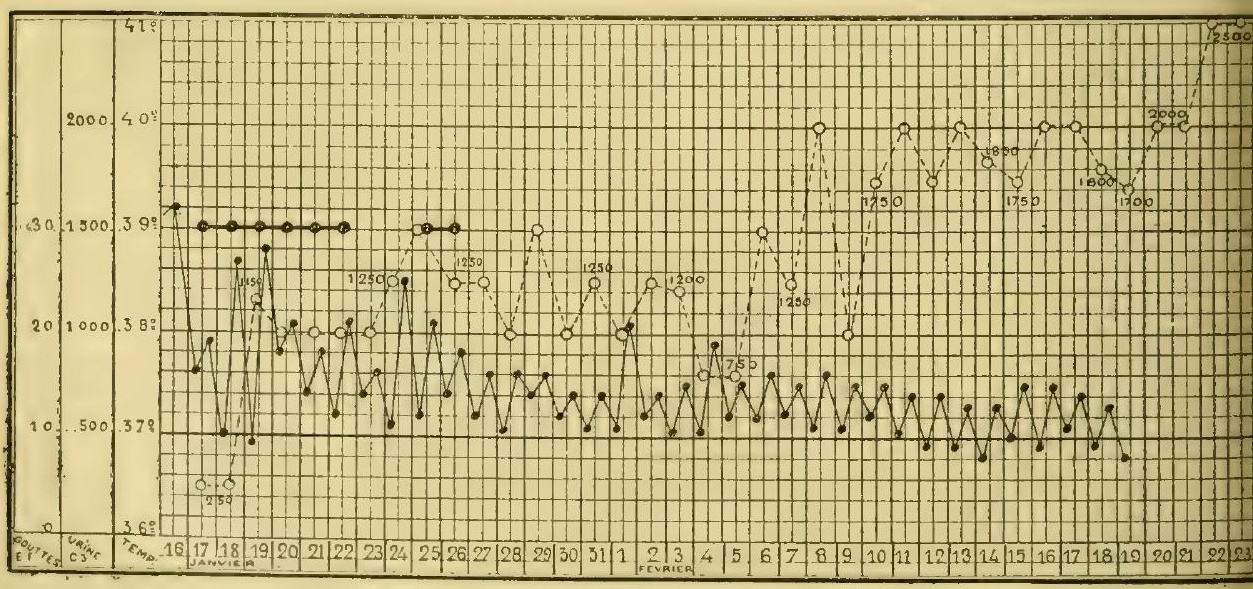

OBS. IX. 
Rem. - L'administration d'extrait fluide a provoqué de suite une augmentation des urines; celles-ci, malgre des oscillations, se maintiennent ì un chiflre élevé. Plusieurs jour's après la cessation du traitement, la diurèse se maintient.

Ons. X. - (T'raitement : lo par la solution d'actéine; $2^{\circ}$ par l'extrait lluirle.)

Le nommé P... entre en décembre à l'hôpital pour une poussée de bronchite aigue.

ANT. héı̇́D. - Pas de renseignements.

ANT. pensond. - A 18 ans, fièvre typhoïde, depuis lors bronchite fréquente. L̇n 1892 le malade a été soigné pour une bronchite gangreneuse. Depuis lors, oppression, expectoration. Signes d'éthylisme avéré.

État actueL. - Depuis quelques semaines, oppression continuelle entremêlée d'accès de faux asthme. Cardiopathie secondaire. A son entrée a l'hôpital le malade présente :

Poitrine en voussure.

Poumons. - Murmure vésiculaire aboli. Râles sibilants et ronllants.

Cœur. - Bruits très sourds, bruit de galop.

Urines. - Normales.

Diagnostic. - Emphysème. Tuberculose fibreuse probable. Bronchite chronique avec poussée de B. aigue.

\section{Traitement :}

\section{Parl'actéine.}

21 decembre. - Potion gommeuse : actéine en solution, 2 centig.

23 décembre. - Crise polyurique le 22. Urée 11 gr. 529 par litre.

24 decembre. - Expectoration un peu moins abondante. Pas de diarrhée, pas de maux d'estomac. Actéine 3 centig.

h. X.1.

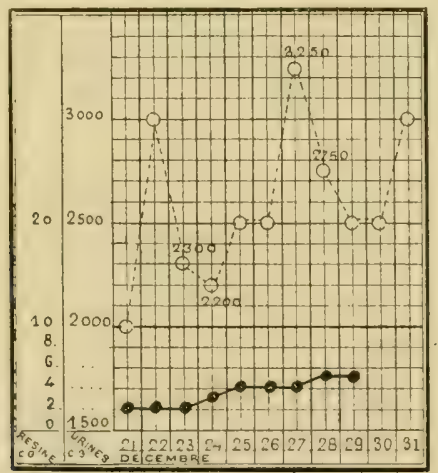


25 décembre. - Même état, actéine 4 centigrammes.

27 dẻcembre. -

28 decembre. - 5 centig. Respiration plus facile. Expectoration bronchitique est moins abondante.

Urẻe $10 \mathrm{gr}, 2$ par litre.

30 décembre. - Suppression de l'actéine. Constipation.

2 janvier. - Urines 2 litres 500. Urée 11 gr. 3 par litre.

$2^{\circ}$ Par l'extrail fluide.

4 janvier. - Extrait fluide

6 janvier. -

8 janvier. -

11 janvier. - 》
$X$ gouttes

XX gouttes.

XXX gouttes.

$\mathrm{XI}$, gouttes.

15 janvier. - Pilules d'ergotine. Sulfate de quinine.

25 janvier. - Théobromine. Suppression de l'actée.

10 février. - Respiration très pénible. Sommeil difficile.

19 févier. - Etouffe toujours et crache beaucoup.

28 février. - Mème état.

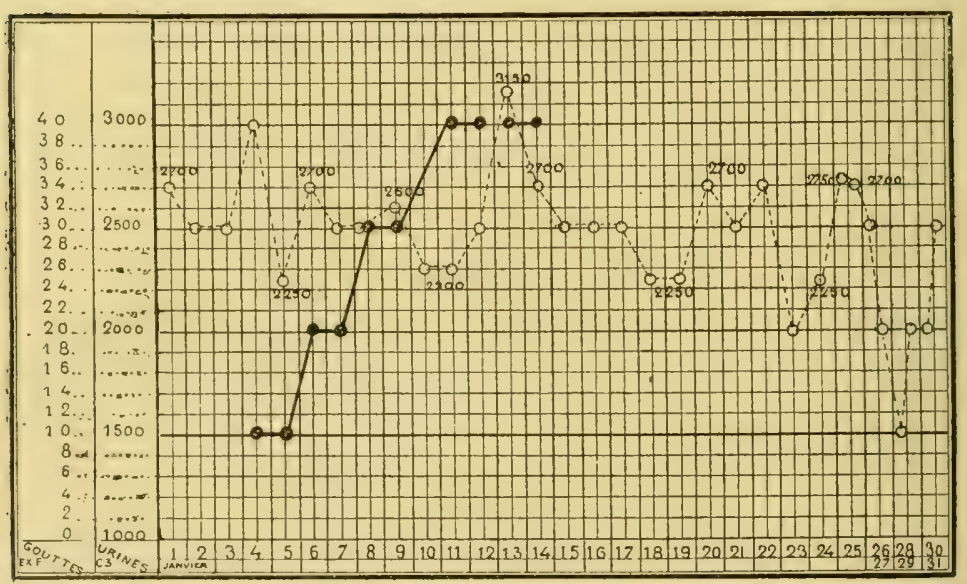

Obs. X. 2.

Rem. - Pendant la durée du traitement par l'actéine, on constate des oscillations périodiques assez nettes dans la courbe d'urine. A mesure que les doses d'actéine croissent, la quantité d'urine croît également.

Dans ce cas, l'action diurétique de l'extrait paraît sensiblement égale à celle de l'actéine. Les oscillations périodiques se poursuirent pendant la seconde partie du traitement, même après sa ces- 
sation; après celte cessation, la courbe se maintient encore, pendant quelques jours, à un niveau assez élevé.

\section{OBs. XI. - (Traitement par la solution d'actéine.)}

Le nommé L... entre à l'hôpital le 7 février 1897, pour accès d'oppression et dyspnée. Peu d’antécédents. Aucune malidie infectieuse. Depuis 3 ans, accès de suffocation.

ÉTAT ACTUEL. - Quelques nodosités arthritiques. Obésité.

Cœur. - Surcharge graisseuse, accidents dyspnéiques.

Poumons. - Nurmure respiratoire presque aboli.

Diagnostic. - Emphysème. Obésité. Surcharge graisseuse du cœur.

Traitement :

8 février. - Actéine en solution 5 centig.

9 février. - " "

10 février. - Crachats hémoptoïques, râles fins disséminés dans tout le poumon, œdème du poumon. Infarctus. Suppression de l'actéine. Urines 1 litre.

\begin{tabular}{|c|}
\hline \\
\hline Acide gallique........... \\
\hline 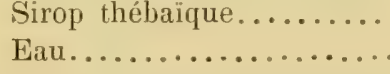 \\
\hline
\end{tabular}

12 février. - Plus de crachats sanglants. Oppression considérable. Urine $900^{c} 3$. Piqùres de caféine.

13 février. - Mort à 11 heures du soir d'une apoplexie pulmonaire.

OBs. XII. - (Traitement par la solution d'actéine.)

M. G... âgé de 72 ans, est entré à l'hôpital le 6 fév. 1897.

ANTÉCÉD. HÉRÉD. - Pas de renseignements.

ANTÉCÉd. PERSON. - Impaludisme.- Gastrite. - Vomissements.

ÉTAT AGTUEL。 - Depuis trois mois, augmentation des vomissements. Accès d'oppression. Articulations douloureuses, surtout aux coudes, quelques déformations de rhumatisme chronique peu avancé, au nireau des doigts, des orteils et des coudes. Artères peu flexueuses mais dures, se plaint surtout de manifestations dyspnéiques et d'un état nauséeux.

Poumons. - Respiration emphysémateuse, quelques râles sibilants et ronflants.

Cour. - Bruits un peu sourds; mais, à la base, claquement très marqué des sigmoïdes de l'aorte.

Foie. - Déborde légèrement les fausses côtes.

Constipation opiniâtre. 
Urines rares. - Pas d'albumine, pas de sucre, urée 11,529 par litre.

Diagnostic. - Emphysème.

T'RAITEMENT:

8 férier. - Actéine en solution 5 centig.

9 févier. - id. Urines, 1,000 grammes:

10 février. - Respire mieux. 2 selles. Urines 1,000 grammes. Actéine $0 \mathrm{gr} .06$ centigr.

11 février. - Le malade semble oppressé, urines $730 \mathrm{~g}$.

12 février. - Respiration plus facile. 2 selles. Urines 1 litre. Vin de guinguina.

14 févier. - Tousse autant, mais respiration plus facile. Urine $850^{\circ 3}$. 1 fövrier. - Urine, 1,2ä0 0 3.

16 février. - Expectoration difficile. Un peu plus d'essoufflement. 1 selle. Suppression de l'actéine.

17 fevrier. - Le malade se sentant bien demande it sortir.

OBS. XII.

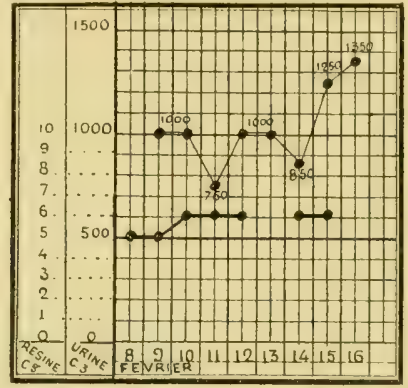

Rem. - Régularité des selles chez un malade ayant, avant l'em ploi de l'actée, une constipation rebelle.

La courbe d'urine présente les mêmes oscillations, presque périodiques, que les précédentes, la diurèse s'accentue après la cessation du médicament.

V. aussi Obs. II et III, IV, VIII, X, XVIII. Emphysème.

\section{DANS TA TUBERCULOSE}

Dès 1823, GARDEN publiait une note relative à l'action de l'Actáa, dans la tuberculose pulmonaire, note d'autant plus intéressante que l'auteur y relate son propre cas.

Peu après l'emploi de ce remède, dit-il, « l'hecticité s'est arrètée, les sueurs nocturnes diminuent, l'expectoration semblable 
à du pus disparaît : la toux devient moins fréquente et moins fatigante.

Mon pouls, qui n'étail jamais au-dessous de 100 à 120 , redevient normal, la douleur de mon coté s'apaise, mes forces et mon appétit renaissent, j'abandonne tous les traitements et me live seulement à un exercice régulier.

Depuis un an j'avais tout essayé : vésicatoires, cautères, etc... ”

Et il ajoute : le Cimicifuga possède certainement " à un haut degré le pouvoir de baisser la tension artérielle et de relever l'organisme. ”

Plus tard, il revient sur le même sujet, pour confirmer son opinion première, par un succès de 30 années.

Heldreti a rapporté 3 cas de phtisie, améliorés considérablement par le Cimicifuga, associè à l'iode. Il est pleinement de l'avis de GARDEn sur son efficacité.

"Nous n'avons jamais usé, dit Davis, de l'A. racemosa seule dans aucune forme des affections pulmonaires, mais nous l'avons vue agir dans la scrofule et la tuberculose, jointe à l'iode.

“ Notre expérience de ce remède ne nous le fait voir ni comme un stimulant, ni comme un modificateur des sécrétions, mais comme un sédatif dans toute la force du terme.»

Brtirgon croit l'actéine utilement employable dans le traitement de la phtisie.

Simpson vante aussi ses bons effets dans la tuberculose pulmonaire.

OBS. XIV. - (Traitement par la poudre d'actéine.)

Le malade entre à l'hôpital le 26 mai 1896.

ANTÉCÉD. HÉRÉD. Père mort à 43 ans d'une bronchite. Mẻre morte à 80 ans.

ANTÉCÉD. PERSON. Ne se rappelle aucune maladie d'enfance, a été éthy lique, mais ne boit plus.

ÉTAT ACTUEL. - Depuis environ un an, étouffement, dyspnée à chaque effort. Toux fréquente la nuit. Mal de gorge et épuisement.

Poumons. - Submatité des 2 sommets en arrière, respiration rude et soufflante. Matité sous la clavicule gauche en avant, craquements 
humides, râles ronflants et sibilants dissséminés dans toute la poitrine. Exprectoration abondante, bronchitique et muco-purulente avec bacilles.

Autres organes normaux.

Diagnostic. - Tuberculose pulmonaire.

Traitement :

27 mai. - Actéine en poudre, 1 pilule.

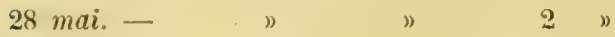

29 mai. - "

30 mai. - " 4 "

31 mai. - " " $"$ "

1 er juin. - Tousse toujours beaucoup. 6 "

2 juin. - Les signes bacillaires sont les mêmes. Plus de râles dans la poitrine. 2 cachets de 5 centig.

3 juin. - Crache moins. 2 cachets de 15 centig.

4 juin. - Même traitement. Maux d'estomac.

כ̆ juin. - Suppression de l'actéine.

OBs. XV. - (Traitement par la poudre d'actéine.)

Victorine D... entre à l'hôpital le 26 mai 1896.

ANTÉCÉD. HÉRÉD. - La malade n'a pas connu ses parents.

ANTÉGÉD. PERSON. - Coqueluche vers 7 ans; variole vers 13 ans; fièvre typhoïle vers 19 ans. Premières règles à 13 ans; depuis, menstruation normale, bien qu'accompagnée de iégère céphalalgie; grossesse et couches pénibles à 25 ans.

HistoIRE DE LA MALADIE :

En 94, bronchite, depuis laquelle toux persistante. Depuis 3 semaines augmentation de la toux. Sueurs nocturnes, fatigue continuelle, anorexie, quelques douleurs vagues.

État actuel :

Poumons. - A gauche, submatité du sommet, expiration rude, quelques râles humides; mais le tout est voilé par de gros râles ronflants et sibilants, disséminés dans toute la poitrine. Crachats muco-purulents. Recherche des bacilles négative.

Cœur. - Rétrécissement mitral dénoté par un dédoublement du

e temps, et un souflle diastolique qui s'entend un peu au-dessus du point d'élection, souftle qui semble avoir pour origine une insulfisance aortique.

Foie. - Peu gros.

Diagnostic. - Tuberculose pulmonaire très probable.

TRAITEMENT :

27 mai. -1 pilule.

28 mai. - Les règles, qui ne s'étaient pas montrées depuis 3 mois, sont apparues; actéine 2 pilules.

29 mai. - 4 pilules. 
31 mai. - Un peu moins de sécheresse dans la poitrine. La malade se trouve soulagée. Elle tousse un jeu moins, mais les crachats n'ont pas

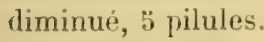

Beaucoup moins de râles que les jours précédents.

ler juin. - La température est tombée; la toux a diminué; l'expectoration est toujours abondante, et on remarque quelques filets de sang dans les crachats, quelque crépitation aux bases. En avant sous la clavicule gauche, expiration rude et prolongée: 6 pilules.

2 juin. -2 pilules, et le soir 1 cachet de 0,15 centigrammes.

3 juin. -2 cachets.

4 juin. - Vives douleurs au creux épigastrique, suppression des cachets.

з̆ juin. - Les douleurs se sont calmées. Amélioration.

Rem. - La réapparition. à la suite du traitement par l'actéine, des règles, qui ne s'étaient pas montrées depuis 3 mois, est à noter.

OBs. XVI. - ('Traitement : $1^{\circ}$ par la solution d'actéine; $2^{\circ}$ par l'extrait fluide.)

La femme A... J... entre à l'hòpital le 28 décembre 1897 pour une hémoptysie abondante.

ANT. HÉRÉD. - Peu de renseignements.

Ant. PEnsonn. - Plusieurs hémoptysies antérieures et fluxions de poitrine.

ÉTAT ACTUEL :

Poumons. - Gauche : Matité et submatité du sommet, expiration lente et prolongée.

Droit : En arrière, grosse caverne, souffle amphorique, craquements, " ramollissement en avant, matité, craquements humides. Bacilles.

Cæur. - Normal.

Urines. - Traces d'albumine.

Diagnostic. - Tuberculose pulmonaire.

Traitement :

$1^{\circ}$ Par la solution d'actéine.

21 décembre. - Arrèt de l'hémoptysie, actéine en solution : 6 centigr.

23 décembre. - La température est tombée. Expectoration peu abondante. Auscultation : Mêmes signes. Actéine en solution : 8 centigr.

24 décembre. - T'races d'albumine, pas de sucre. Urée : 12 gr. 81 par litre.

25 décembre. - La malade est constipée. A très légèrement souffert de l'estomac. Actéine en solution : 10 centigr. 
27 décembre. - Expectoration muco-purulente, lémoptysic le 26. Petite crise polyurique. Urée : 14 gr., 5 par litre.

28 décembre. - L'hémoptysie est arrêtée. La malade se sent mieux. Actéine en solution : 10 centigr.

\section{$2 \circ$ Par l'extrait fluide.}

31 décembre. - Même état.

3 janvier. - IIémoptysies le $1^{\mathrm{er}}$ et le 2. La malade se sent très faible, potion d'ergotine.

4 janvier. - Abaissement de la température. Extrait fluide $\mathrm{X}$ gouttes

6 janvier. - Etat nauséeux, diminution de l'expectoration. Extrait lluide $\mathrm{X}$ gouttes.

8 janvier. - Extrait fluide XX gouttes.

10 janvier. - Respiration plus facile, même traitement.

13 janvier. - Bien. Urée, 6 gr. 405 . Suppression de l'actée.

20 janvier. - Etat général meilleur.

22 janvier. - Crise d'etouffement.

20 jonvier. - Toux sèche en quintes.

3 février. - Très grande oppression, nuit mauvaise. Teinture d'iode, ventouses.

10 février. - Ne va pas mal.

19 février. - Malade moins bien. Suppression des crachats, sensation de sécheressse à la gorge.

23 fevrier. - Nouvelle hémoptysie, potion à l'acide gallique; potion d'ergotine.

28 février. - Etat général bon.

V. aussi Observ. VII. Tuberculose prolıable.

Observ. X. T. fibreuse probable.

Rem. - Dans l'administration de l'Actée à des tuberculeux, l'étal du tube digestif doit être pris en très haute consideration. Chez les Luberculeux, les voies digestives sont altérées, parfois dès le début de la maladie. Le plus grand souci du médecin doit être de surveiller l'alimentation et le bon état de l'estomac; il pourrait être dangereux de les soumettre à l'action d'un médicament irritant pour le tube digestif, tel que l'Actée.

La même réflexion s'applique à nombre de bronchites dites sclé- 
reuses, derrière lesqueltes se cache la bacillose. Celte reserve est d'autant plus justifiée que l'action de tout irritant du tube digestif s'exerce avec beaucoup plus de violence, quand il existe une inflammation de la muequeuse digestive; à tel point que la tolérance pour un tel médicament peut neetre jamais obtenue, chez une personne dont le tube digestif est malade.

DANS IES AFFEGTIONS DE L'APPAREI GIRCULATOIRE

L'Actée a été vantée par PaTTox contre les bourdonnements d'oreilles, évidemment à titre de modificateur de la circulation auriculaire. "Dans les cas simples, dit-il, on obtient souvent une cure complète ou une amélioration marquér : quelquefois le mieux persiste, tant que le remède est employé; quelquefois la cure est radicale.

"Dans les cas graves (je parle de ceux relatifs aux lésions de l'oreille moyenne ou interne), je n'ai jamais trouvé aucune observation ayant trait à l'utilité de ce médicament, mais il devrait être recommandé d'en faire l'essai, et de le donner dès le début de l'affection. »

On a comparé l'action de l'actée sur la circulation à celle de la digitale. (Lauder-Brunton.)

Pour la plupart des auteurs américains, l'Actée se comporterail comme "un sédatif du système artériel ».

Davis et Jonnsox affirment qu'à doses thérapeutiques elle diminue constamment la force et la fréquence du pouls, et fait tomber la pression artérielle.

Gardex la considère plutôt comme un tonique du système circulatoire.

DANS L'ARTÉRIO-SCLÉROSE

OBS. XVII, - (Traitement : $1^{\circ}$ par l'extrait fluide;

$2^{\circ}$ par la solution d'actéine.)

Ime C. entre à l'hòpital le 18 janvier.

Axtécéd. hÉnéD. - Père mort d'apoplexie, mère morte âgée.

ANTÉCÉd, PERsox, - Premières règles à 13 ans depuis normales. Aléno- 
pause it $: i 2$ ans; 6 cnfants. Dejuis la ménopause, eczéma. En 1886, hémiplégie avec aphasie ayant duré 3 mois.

Histoire dE LA MALAdIE:

Crises d'oppression et étouffement depuis 3 à 4 mois, s'accompagnant de sueurs. Apres chaque crise sommeil difficile. Expectoration abondante. Pas de varices. Pas de nodosités arthritiques. Quelques petits signes de brightisme, pollakiurie, doigt mort, etc.

ETAT ACTUEL. - Constipation opiniâtre.

Poumons. - Diminution du murmure vésiculaire, quelques râles ronflants et sibilants.

Cœur. - Bruits bien frappés. Pas de souffle, bruit de galop. Radiale dure, pouls légèrement bondissant. Temporale non sinueuse.

Rein. - Ni albumine, ni sucre.

Diagnostic. - Artério-sclérose.

TRAITEMENT :

10 Par l'extrait fluide.

19 janvier. - Extrait fluide XXX gouttes.

25 janvier. - Même traitement, respiration plus facile, va plus facilement à la selle.

30 janvier. - Même état, grande faiblessé; kola et coca.

2. Par la solution d'actéine.

$1^{\text {er }}$ février. - La malade se trouve très bien. Actéine en solution 5 centig.

5 février. - Tête lourde, léger malaise.

12 février. - Étouffement. Selles régulières.

KI. Benzoate de soude.

14 février. - Va un peu mieux, dort bien. Suppression de l'actée.

17 février. - Un peu moins bien.

19 février. - Crise d'étouffement. Constipution.

21 février. - Iodure de potassium. Benzoate de soude. Grande constipation.

24 février. - Même état.

Rem. - Le résultat le plus net du traitement a été la régularisaion des selles; à ce point de vue l'actéine a agi aussi efficacement que l'extrait fluide. Sitot la cessation du traitement, la constipation a reparu.

OBS. XVIII. - (Traitement : $1^{0}$ par la solution d'actéine; $2^{\circ}$ par l'extrait fluide.)

La femme G... B... entre à l'hôpital en juillet 1896.

ANTÉCÉD, HÉRÉD. - Père mort de bronchite, mère rhumatisante. 
AntécÉd. PEnson. - Premières règles à 12 ans $1 / 2$, depuis régulières, normales, ménopause à 46 ans; dans sa jeunesse; variole et ictère; depuis la ménopause, rhumes et bronchites, fréquents et longs.

ÉTAT ACTUEL :

Poumons. - Sonorité; murmure respiratoire étouffé, râles ronflants et sibilants; expectoration abondante muco-purulente.

Cœur. - Léger souffle systolique à la base, artério-sclérose de l'aorte; oppression, lèvres violacées, pseudo-asthme.

App. digestif. - Foie cardiaque, constipation rebelle.

Urines. - Foncées, sédimenteuses, peu abondantes; ni sucre, ni albumine; urée 13 gr. 43 par litre.

Diagnostic. - Artériosclérose, arthritisme, cardiopathie, emphysème.

Traitement :

1. Par la solution d'actéine.

De juillet à décembre. - Saignées, purgations fréquentes; la malade est très oppressée, cyanosée, elle tousse et crache beaucoup.

9 décembre. - Actéine en solution, 1 centigramme.

10 dícembre. - Toux et crachats non modifiés, état nauséeux, même traitement.

13 décembre. - Respiration plus facile, même traitement.

16 décembre. - État plutôt meilleur. Peu de râles, expectoration moins abondante; urines, $1 / 2$ litre, urée, 13 gr. 3 par litre; actéine en solution, 2 centigrammes.

18 décembre. - Journée du 17 assez bonne, malgré une crise d'oppression. Pour la première fois augmentation de la diurèse, 1 litre. Solution d'actéine, 2 centigr. $1 / 2$.

20 décembre. - Diurèse établie, mème traitement.

21 décembre. - La malade se plaint d'une sensation de brûlure au niveau du creux épigastrique. Suppression de l'actéine.

23 décembre - La malade souffre du ventre et de l'estomac, la douleur est due à l'asystolie hépatique; reprise de l'actéine, 2 centigrammes.

2ə̃ décembre. - Augmentation de l'urine qui remonte à 1.500 grammes; urines claires. La malade a bien dormi. Elle se sent mieux malgré une toux fréquente; aucune douleur d'estomac. Actéine, 2 centigrammes.

27 décembre. - L'action diurétique ne se mannent pas. Urines : $20^{0}{ }^{3}$. Constipation, oppression. Actéine en solution, 3 centigr.

28 décembre. - Urines : $850^{\text {c3 }}$. Léger nuage d'albumine. La malade tousse et crache beaucoup. Pas de selles. Actéine en solution 4 centigr. Douleurs gastriques, état nauséeux.

30 décembre. - Urée : 16 gr. par litre. Suppression de l'actéine.

31 décembre. - La malade étouffe et se plaint de l'estomac. 
$2^{\circ}$ Par l'extrait fluide.

\& janvier 189\%. - Expectoration bronchique, dyspnée.

Extrait fluide.................. X gouttes.

6 janvier. - Vomissements dans la nuit du \& au 5, pas de douleurs d'estomac.

Extrait fluide.................. XX gouttes.

8 janvier. - Toux opiniâtre, 1 selle.

10 janvier. - Oppression très douloureuse, expectoration muco-purulente, crachats sanguinolents.

$\left\{\begin{array}{l}\text { Ergotine } \\ \text { Sirop de digitale }\end{array}\right.$

$\left\{\begin{array}{l}\text { calomel. } \\ \text { scamonnée. }\end{array}\right.$

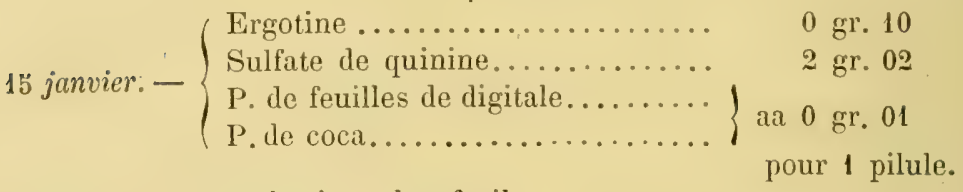

18 janvier. - Respiration plus facile.

20 janvier. - Strophantus $3 \mathrm{gr}$.

22 janvier. - Même traitement. Un peu de mieux.

29 janvier. - Oppression très douloureuse, ventouses.

$1^{\text {er }}$ février. - Même état.

10 février. - Sirop de belladone......... $30 \mathrm{gr}$.

Teinture de lobélie........... $2 \mathrm{gr}$.

Alcoolature d'aconit............. XX gouttes.

20 février. - Respiration de plus en plus difficile, palpitations, exophtalmie.

28 février. - Un peu calmée par la digitale, mais même état.

Rem. - Dans ce cas, la digitale a réussi à provoquer une amélioration, alors que l'Actée est restée impuissante (l'actéine tout comme l'extrait fluide).

L'Actée a réussi à établir la diurèse, mais en pleine crise polyurique est survenue une asystolie hépatique et rénale; sous l'influence de cetle asystolie, la diurèse s'est arrêtée. La conclusion à tirer, semble-t-il, de cetle observation est que l'Actée doit provoquer la diurèse, non par suite de son élimination par le rein, mais en modifiant la circulation rénale.

V. aussi obs. III, XI, surcharge graisseuse du ccur. 
Obs. IV, IX, artério-sclérose.

Obs. $X V$, rétrécissement mitral, insuffisance aortique.

Obs. XIX, artério-sclẻrose généralisée, aortite.

DANS LES AFHECTIONS NERVEUSES

L'Actée a été vantíe contre diverses affections nerveuses, et en particulier contre l'

\section{Épilepsie.}

Nous arons eu l'occasion de l'essayer sur une malade, alteinte de mal comitial.

OBS. XIX. — ('Traitement par l'extrait fluide.)

La nommée B..., âgée de 71 ans, entre à l'hôpital en octobre 96.

ANTÉGÉD. HÉRÉD. - Mère morte accidentellement à 51 ans, père à 79 ans d'une bronchite, mais le père et 5 frères et sœurs de la malade semblent atteints d'accidents comitiaux.

ANTÉCÉD. PERSON. - Enfance normale, menstruation établie à 15 ans, sans déterminer les accidents comitiaux qui n'apparaissent que quelque temps après. Les premières attaques sont nocturnes, et ne laissent aucun souvenir à la malade. Celle-ci se marie à 19 ans, a 3 enfants, qui meurent au moment de la dentition d'accidents convulsifs. Ménopause à 40 ans; attaques plus fréquentes et apparitions de troubles gastriques et circulatoires. La malade est une arthritique ayant eu plusieurs poussées de rhumatisme. En juillet 1896, grave épistaxis suivie d'ictère.

État actuel:

App. circulatoire. - Artério-sclérose généralisée. Souffle systolique de la base.

Cœur. - Aortite : souffle systoliquede la pointe. Pouls lent, petit, facilement dépressible.

App. respiratoire. - Normal.

App. digestif. - Troubles gastriques assez vagues. Anorexie, constipation. Parfois ténesme rectal.

App. uro-génital. - Urines normales; un peu de cystite. Après la ménopause, leucorrhée.

Système nerveux - Céphalalgies fréquentes, sans localisation spéciale. La pupille réagit normalement. Pas de points hystériques. 
Attaques. - Pas de prodromes éloignés: aura constante, motrice et viscérale, débutant par le pouce droit qui est le siège de tremblement et de chatouillement. En même temps, d'une facon constante, vif besoin d'aller à la selle, suivi parfois d'évacuation abondante. L'attaque suit l'aura. Ellle se produit la ‘nuit. Palpitations, avec douleurs localisées à la paroi tlıoracipue du còté gauche, refroidissement'des extrémités, frissons, spasmes, troubles visuels : fantômes, hallucination, parfois diplopie. Troubles psychiques i peu près nuls. Jamais de perte deconnaissance ni d'accidents convulsifs, durée de l'attaque 1 h. 1/2 à 2 heures.

DiagnostiG. - Epilepsie.

Traitement :

29 octobre. - Attaque à 8 heures du matin; urines $820^{\mathrm{cm} 3}$. Dens. de l'urine 1,013 , ni albumine ni sucre.

D'octobre a janvier : doul. hépatiques vagues; on essaie la solution d'actéine jointe à la lactose. Médicament mal toléré : on le supprime.

fer janvier Miême attaque.

3 janvier. - Extrait fluide.............. $\mathrm{X}$ gouttes.

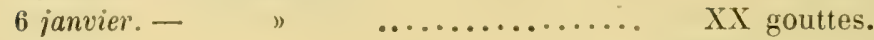

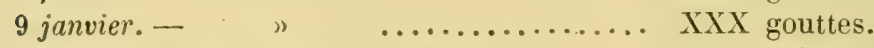

La malade, ordinairement constipée, va à la selle; depuis 2 jours pas de diarrhée, pas de douleurs gastriques.

10 janvier. - Crise différente des crises habituelles; douleurs dans le bas-ventre; suspension des urines pendant 3 heures, puis accès de polyurie (2 litres). Malaise général, mais absence de la tachycardie, de l'angoisse, des frayeurs habituelles, quelques coliques intestinales sans diarrhée.

11 janvier. - Extrait fluide XXX gouttes.

13 janvier. - Nouvelle crise, débutant par un malaise général et un besoin d'aller à la garde-robe; 3 selles non diarrhéiques. C'est la $1^{\text {re }}$ fois que les crises sont aussi rapprochées. Pulsations cardiaques tombant de 138 à 88 apres l'attaque, tachycardie, polyurie. Suppression de l'ext. fluide.

15 janvier. - Pas d'actée.

20 janvier. - Velléité de crise, pas d'actée.

22 janvier. - Crise durant 5 heures : douleurs dans le bas-ventre ; impossibilité de la miction, douleur de tête, angoisse. Bromure.

24 janvier. - Le bromure apporte quelque soulagement.

29 janvier. - Pas de crise, mais cystite; la malade se trouve plutôt moins . bien. Lavages de la vessie; bromure.

31 janvier. - Attaque commençant par une rétention urine et ne cessant que par la miction.

8 février. - Nouvelle crise identique à la dernière.

10 février. - Attaque de paralysie sans perte de connaissance, ventouses scarifiées.

12 février. - Figure déviée à droite, hémiplégie gauche. Aucune perte de connaissance ni de la parole. İschémie cérébrale momentanée. 
19 févier. - Douleurs de ventre. Céphalée. Etat moins bon. 24 fivrier. - Crise d'oppression. Valérianate d'ammoniaque. 28 février. - Malade fatiguée. Se plaint beaucoup.

OBS. XIX.

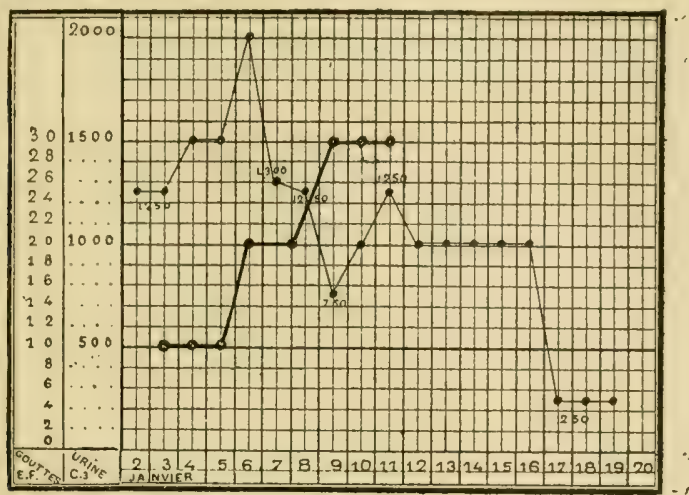

Remarque. - L'Actée s'est encore montrée ici efficace à régulariser les selles; la constipation opiniâtre dont souffrait la malade a disparu par son emploi.

L'actéine, mal supportée par un tube digestif irritable, a pu être remplacée, sans inconvénient, par l'extrait fluide.

Après un traitement de quelques jours par l'Actée, les troubles circulatoires, dus à l'artério-sclérose, n’ont pas été améliorés, puisqu'une ischémie cérébrale temporaire a pu se produire.

Il semble que l'Actée a réveillé la susceptibilité intestinale; sous son influence, les attaques, dont le point de départ est une aura intestinale ont paru se multiplier.

Il y a là un enseignement à retenir.

L'Actée est formellement contre-indiquée dans les cas d'épilepsie, où l'aura siège dans le tube digestif.

Dans le cas unique où nous avons pu l'expérimenter, loin de répondre à la réputation qui lui est faite, elle s'est plutôt montrée nuisible.

\section{Hystẻrie.}

Signalons l'emploi que l'on fait de l'Actée à haute dose contre l'hystérie (MILLARD). 
Riscier la prétend efficace contre les migraines d'origine hystérique.

C'est sans doute de troubles hystériques qu'étaient affectés les malades dont Crair: Balfour rapporte l'histoire. Citons les observations de cet auteur en nous dispensant de les discuter.

"Il y a quelques mois, je fus appelé près d'une fillette de $\mathbf{1 2}$ ans, et qu'on me disait très malade.

Sa mère m'expliqua qu'un jour, six mois environ avant ma visite, et alors qu'elle semblait en pleine santé, elle s'était plaint d'une douleur dans le bras et dans la jambe, en même temps que d’un malaise général.

Depuis ce moment, son état s'était graduellement aggravé. On l'avait tout d'abord traitée sans succès pour une névralgie, un rhumatisme, puis pour une affection de la colonne vertébrale, et on lui conseillait l'immobilité.

Elle se plaignait de douleurs dans les jambes, et dans l'épine dorsale, ainsi que d'une grande sensibilité de l'abdomen.

Elle remuait ses l)ras facilement, mais ses jambes lentement, et accusait une douleur si on les touchait.

Elle était capable à la rigueur de marcher et de se tenir debout, mais elle ne dormait pas, et accusait des étourdissements intenses dans l'obscurité, surtout quand on la laissait seule.

J'examinai à nu la colonne vertébrale: aucune déformation, mais la petite malade criait si on lui touchait le dos, les jambes ou le ventre. On ne pouvait me renseigner beaucoup sur la famille; cependant je pensai qu'il ne s'agıssait pas d'une affection de la colonne vertébrale, mais d'une névralgie hystérique, d'autant plus que la mère et la grand'-mère de la malarle souffraient de migraines, ainsi qu'une sœur aînée.

Pour commencer, j'ordonnai $\mathrm{X}$ gouttes de teinture d'Actæa toutes les six heures, additionnées de $\mathrm{X}$ gouttes de bromure d'ammonium, et à moins de six heures d'intervalle, si les douleurs revenaient violentes.

Deux jours après je la vis : elle avait mieux dormi, mais elle accusait une douleur du ventre et surtout du bas-ventre.

J'ordonnai des cataplasmes sinapisés sur les deux ovaires, remplacés aussi souvent que le permettrait l'état de la peau. L'effet fut considérable : les étourdissements disparurent.

Trois semaines après, j'ajoutai à l'Actæa une petite dose desirop d'Easton, et six mois plus tard, la petite malade marchait avec toutes les apparences de la guérison.

Elle fut envoyée à la campagne, pour changer d'air, et tout traitement fut abandonné.

Trois mois après, à son retour, elle était abolument bien, n’ayant ni douleur, ni étourdissement, ni insomnie. J'avertis la mère que si quelques douleurs réapparaissaient au moment de la formation, l'Actæa racemosa en aurait vite raison. 
$\Lambda$ ce cas si probant, peut se joindre celui d'une jeune femme souffrant d'une névralgie pubienne depuis la naissance de son dernier enfant. La douleur était si forte que les os semblaient atteints.

$\mathrm{X}$ gouttes de teinture d'Actæa pendant quelques jours amenèrent la guérison. ")

\section{Dans la chorée.}

Les’ premières notes relatives à l'action de l'Actxa racemosa dans la chorée, furent publiées par Joung, qui donnait le compte rendu de plusieurs cas, promptement et efficacement guéris par son. seul usage.

Les faits suivants, publiés par DAvis, parleraient, suivant lui, dans le même sens.

Nous arons tenu à rapporter cette observatton en entier, pour qu'on puisse juger, à sa simple lecture, de sa valeur démonstrative.

" Je me rappelle, dit-il, avoir vu, il y a deux ans, un jeune homme, habitant une campagne arriérée, et atteint de la plus forte chorée que j'aie jamais été appelé à constater. Les secousses musculaires étaient si violentes qu'il pouvait à peine marcher sans l'aide de 2 ou 3 personnes, et elles se répétaient si frérquemment que le malade, depuis plusieurs semaines, n'avait pu prendre aucun repos.

Les médecins du voisinage avaient tout essayé : émétiques, calmants, antispasmodiques; tout avait été impuissant.

Le cas était dù à un refroidissement : un examen attentif me fit reconnaitre une inflammation locale.

J'ordonnai immédiatement une décoction de racines de Cimicifuga, et une vessie d'eau froide placée sur l'occiput, et renouvelée toutes les heures.

36 heures après, je trouvai mon malade tranquillement couché dans son lit, avec un pouls ne dépassant pas 60 pulsations par minute.

L'eau froide fut interrompue et la décoction donnée moins fréquemment. Il n'y eut aucun retour de l'affection, et en 8 ou 10 jours le jeune homme retournait a son travail.

Quelle part doit revenir à l'eau froide ou au Cimicifuga? Que le lecteur juge!

Nous pensons que l'eau agit localement et le Cimicifuga sur l'état général ».

J.-J. Sumpox dit avoir constaté, au début d'une chorée d'origine rhumalismale, l'efficacité très spéciale de l'Actée, efficacité qu'il croit pouvoir attribuer aux propriétés sédatives de la plante.

Plus récemment, F. R. Millard exprime son avis en ces termes: 
"Si on se reporte à la liste complète des agents thérapeutiques employés contre la chorée, on reconnaît que le Cimicifuga est beaucoup trop négligé.

Depuis ces quinze dernières années, tous les praticiens de ma connaissance ont déclaré que le Cimicifugd était aussi actif, dans les cas de chorée survenant dans l'enfance ou dans la puberté, que la quinine dans les fièvres intermittentes : il demeure facilement le premier de tous les remèdes antichoréiques.

Mon expérience personnelle corrobore entièrement cette opinion.

Les seules déceptions que j'ai eues se rapportaient à des cas de chorée, survenus à la suile d'une attaque d'endocardite rhumatismale aiguë.

Dans la chorée ordinaire, une anesthésie modérée peut enrayer les mouvements, pendant un temps plus ou moins long.

Dans les cas graves, l'anesthésie chirurgicale la plus profonde arrête en général les mouvements du trone et des jambes, mais n'arrête pas le tremblement de certains muscles ou de certains groupes de muscles, et avant que la connaissance soit redevenue complète, l'agitation est souvent plus forte que jamais.

Il est naturel de penser que plus l'attaque sera courte - moins le cœur courra risque d'être lésé dans la suite; aussi un mẻdicament qui arrêtera les symptômes en quelques jours, devra-t-il toujours être préféré à un traitement qui assurera le même résultat en 4 ou 6 semaines.

Quand on considère la rapidité et la sùreté avec lesquelles la chorée est combattue par l'Actée, sans aucun danger ou aucun effet funeste (le seul inconvénient peut être un assez léger mal de tête de courte durée), et si l'on compare ce traitement à celui par l'arsenic, qui doit être longtemps continué, qui est incertain, et duquel résultent fréquemment des dérangements gastriques et des troubles nerveux multiples, il parait étrange que des praticiens continuent ì nous dire: "Le traitement par l'arsenic est le meilleur qui puisse être employé. »

Évidemment personne n'a comparé les deux traitements pour arriver à cette conclusion. 
La seule raison que j’imagine pour la persistance en faveur de l'arsenic, c'est qu'aucun maître n'a recommandé Ie Cimicifuga.

Dès que les manifestations choréiques ont cessé, la dose doit être un peu diminuée; mais le remède sera continué quelque temps, moins pour prévenir le retour de la maladie que son action sur le coeur. "

RINGER dit avoir constaté « que l'Actée n'est efficace que dans lachorée d'origine rhumatismale $v$, et il ajoute: "Ce médicamentest connu depuis des siècles, par les Indiens, comme sprécifique de la chorie » (!)

Remaique. - Il est à remarquer que les propriétés attribuées par les auteurs à l'Actée tendraient, a priori, à la rapprocher, du groupe des antimoniaux, et que le tartre stibió a été vanté contre la chorée (BERGERoN).

Nous n'avons pas eu l'occasion d'expérimenter l'Actée contre la chorée chez l'homme, mais nous avons soumis à un traitement journalier, de plusieurs semaines, par l'extrait fluide d'Actée (30 gouttes), une chienne atteinte de chorée.

Cette affection consécutive à la maladie infectieuse du jeune âge, si fréquente chez le chien, se prolongeait depuis trois ans, avec une intensité croissante; sa principale manifestation était un mouvement choréique de la patte antérieure droite; sous l'influence du traitement, l'affection n'a été en rien améliorée (mais l'état général s'est maintenu excellent).

L'enregistremeut graphique des secousses choréiques du membre atteint faites avant, pendant et après le traitement, n'ont montré aucune modification, même légère, dans le rylhme et l'amplitude des mouvements.

Ajoutons qu'il serait d'ailleurs imprudent de conclure de la chorée du chien à celle de l'homme; l'origine infectieuse de la première est aujourd'hui bien établie, celle de la seconde n'est que probable. Nous savons que le mouvement choréique du chien est dû à une altération des centres médullaires, consécutive à une action microbienne [Triboulet (I)]. Quoi qu'il en soit, l'Actée qui

(1) 189.̈. Triboulet (H.). La chorée du chien. (Trav, du labor. de M. Ch. Richet, t. III, p. 175.) 
pourrait agir dans celte affection, si elle était douée de propriétés modératrices réflexes, s'y montre totalement inefficace.

\section{DANS LES AFFECTIONS RUUMATISMALES}

\section{$1^{\circ}$ Rhumatisme articulaire aigu.}

Dès 1838, N. S. Davis rapportait les expériences de Jonnson, relativement à l'action de l'Actée dans le rhumatisme articulaire: "Johnson, dit-il, nous a fait savoir qu'à différentes reprises, et dans plus de vingt cas de fièvre et d'attaques rhumatismales revêtant les formes les plus graves, il a employé le Cimicifuga. Les résultats ont été satisfaisants au plus haut point; tout vestige de l'affection disparaissant au bout de 2,8 ou 10 jours, sans produire le moindre symptôme fàcheux. Ces essais ont été répétés par moi-même et d'autres, au point que nous n'ayons plus de doute sur l'efficacité du Cimicifuga au début de l'attaque rhumatismale, et que nous le considérions comme un préservatif compararable à celui de la vaccination pour la variole (!!). »

Jomsson a constaté que les cas les plus aigus et les plus graves (qu'il ait jamais observés cédaient sous l'influence du Cimicifuga, non seulement plus promptement, mais encore plus parfaitement, et sans la moindre trace de danger pour les autres organes, que sous l'influence d'aucun autre médicament.

"Les seuls effets appréciables de cette médication, dit-il, sont la diminution de la force et de la fréquence du pouls; la clisparition de l'inflammation et de la douleur articulaires, ainsi que cles vertiges.

Nous avons été fort surpris que quelques praticiens aient usé du Cimicifuga dans le rhumatisme, sans obtenir les mêmes effets. Mais dans tous les cas oủ nous avons pu nous informer, nous avons trouvé qu'il avait été commis quelques erreurs, soit dans l'espèce de la plante employée, soit dans la période de l'affection où le Cimicifuga est surtout efficace.

De ce que le Cimicifuga a été considéré par les écrivains médicaux comme un stimulant et un excitant des sécrétions, on a conclu qu'il ne devait être applicable que dans les 
formes subaiguës, chroniques, ou tout au plus ì la fin de la période aiguë du rhumatisme; alor's que c'est principalement dans ces cas que son action a le moins de valeur(!)

Son pouvoir curatif dépendant (selon nous) entièrement de l'influence sédative qu'il exerce sur les nerfs de lin vie organique, le Cimicifuga peut surtout être énergique, quand il est administré au début de l'attaque, et avant la formation des dépôts fibrineux, autour des ligaments et des articulations touchées dépòts qui sont généralement formés à la fin des accès aigus), et cncore plus dans la forme chronique du rhumatisme.

C'est surtout dans la forme aiguë du rliumatisme que le pouroir curatif du Cimicifuga est démontré. Et vraiment, nous pouvons dire que le rhumatisme le plus aigu et le plus manifeste cessera sous l'action de ce remèdle.

De 30 à 60 gouttes de teinture ou 20 grammes de racine mise en poudre seront administrés à un adulte toutes les 2 heures jusqu'a l'amélioration (DAvis). "

Rivger aurait aussi employé l'Actée dans le rhumatisme aigu, et l'aurail vu atténuer rapidement la douleur, surtout dans les cas analogues à ceux-ci.

"Un malade est d'abord inquiété par une douleur, d'apparence rhumatismale, mais sans fièvre. Bientôt l'affection se localise au poignet ou à la main; les tissus s'épaississent, les os du poignet sont gonflés, jusqu'à ce que les mouvements disparaissent et le membre devient inutile. La chaleur engourdit la douleur qui cesse la nuit. L'attaque présente les caractères du rhumatisme blennorrhagique, quoiqu'il n'y ait pas trace de blennorrhagie.

"J'ai plusieurs fois observé que, dans ces cas, l'Actée agit presque instantanément, alors que KI et les autres remèdes sont essayés en rain : la douleur disparait, et les jointures redeviennent souples et utiles. $)$

Sumpox aurait également eu à se louer de l'Actée, dans le rhumatisme articulaire aigu.

Mac Doxald aurait employé l'Actée, avec un égal succès, dans toutes les formes aiguës de rhumatisme articulaire. 
Dans les cas subaigus, dit-il, elle agit avec une remarquable rapidite. Dans tous les cas oì, soit par diathèse rhumatismale, soit par l'habitation dans un pays humide, soit par un refroidissement qui, au lieu de donner un rhume légrer, provoque une douleur rhumatismale aux grens prédisposés, l'Aciée est très satisfaisante et salutaire.

Mais c'est surtout dans les formes plus graves que ses bons effets ont été reconnus. Deux observations de ce genre me frappèrent, en mème temps, au printemps dernier. L'une se rapportait à un garçon de 9 ans, l'autre à un homme de 40 ans! Dans l'un et l'autre cas, les désordres s'aggravèrent jusqu'à ce que l'Actée eût été donnée. La dose employée variait de 20 à 40 gouttes, administrées trois fois par jour.

Tout récemment, Hewelke a employé l'Actée sous forme de teinture (à la dose de IV gouttes, toutes les deux heures, c'està-dire de à XL-L gouttes par jour), dans sept cas de rhumatisme articulaire aigu. Il aurait obtenu sept succès.

Seul Lauder Brunton estime que l'expérience n'a pas confirmé l'efficacité de l'Actée dans le rhumatisme aigu.

N'ayant pas d'expérience personnelle à ce sujet, contentonsnous d'enregistrer l'opinion des auteurs. Mais est-il vraiment bien utile de chercher, dans le rhumatisme articulaire aigu, un succédané au salicylate de soude?

\section{$2^{\circ}$ Rhumatisme chronique. - Arthritisme. - Nẻvralgies.}

C'est aussi contre les affections rhumatismales chroniques que l'Actée a été essayée.

Dans les névralgies idiopathiques, spécialement dans les névralgies faciales, le Cimicifuga agira plus souvent et avec plus de succès qu'aucun autre remède (Gatch-CarEY).

Srmpson aurait vu des cas de lumbago rapidement guéris par l'usage de l'Actée. Si l'opinion de HARLer, sur sa ressemblance d'action entre l'Actée et le Colchique, était justifiée, elle serait digne d'être essayée contre les manifestations goutteuses. Mais les essais physiologiques ne décèlent guére semblable analogie d'action. 
"Dans le lumbago et la sciatique, dit Rryger, son eflet est plus prompt que celui d'aucun autre remède.

J'ai essayé celte plante, avec beaucoup de persévérance, dans la sciatique, le lumbago, et les cas de rhumatisme chronique, dans lesquels les tendons, les muscles ou les articulations sont très douloureux au mouvement, et aussi dans les cass de grande raideur des articulations, mais arec peu ou point de succès. Au contraire, mon ami Bartlett a employé ce remède dans les mèmes cas avec le plus grand succès. Entre mes mains, cependant, ce remède a eu des résultats heureux dans certaines formes de rhumatisme chronique, par exemple, quand les jointures sont très raides et bridées.

Les jointures, dans ce cas, n'étaient pas, il est vrai, gonflées, et la douleur allait de l'une à l'autre sans se fixer profondément. Les crampes douloureuses des jambes, qui interrompent le sommeil, qui sont aggravées par le temps froid et humide et par certains vents, sont aussi justiciables de l'Actée. Dans ces cas, outre son action narcotique, l'Actée détermine souvent une diminution des crampes et de la douleur, et assure le repos et le sommeil. Dans bien des cas, ce remède peut soulager l'arthritisme. "

OBs. XX. - ('Traitement par l'extrait fluide).

La femme L... entre à l'hôpital pour des douleurs.

ANTÉcÉD. HÉnÉD. - Père mort d'excès alcooliques, mère morte à 80 ans.

ANTÉcÉd. PEnSON. - Premières règles à 14 ans, régulières, abondantes, 4 enfants. Ménopause à 50 ans. A ce moment, coliques hépatiques suivies d'ictère. Depuis 3 ans lassitude dans les membres inférieurs et douleurs dans les genoux. Dans la dernière quinzaine de décembre, coliques hép., suivies d'ictère.

État actuel. - Obésité datant de la ménopause. La malade garde le lit parce que ses jambes sont raides. Articulations pas déformées, mais des deux còtés douleurs passagères, très vives, dans le cou-de-pied et les genoux. Pas de craquements : réflexes conservés. Un peu de rétraction palmaire du côté droit.

Cœur. - Souflle systolique à la base de l'aorte et souflle systolique mitral. Pouls petit et irrégulier.

Poumons. - Respiration emphysémateuse. 
Urine. - Pas d'albumine, pas de sucre. Urée 55,125 par litre.

Diagnostic. - Rhumatisme chronique, arthritisme, lithiase biliaire.

TRAITEMENT :

4 janvier. - X gouttes extrait fluide.

6 janvier. - Se sent un peu mieux. Jambes moins douloureuses, quoique raides. Aucune douleur d'estomac, ni vomissements, ni purgation. Extrait fluide $\mathbf{X} \boldsymbol{X}$ gouttes.

8 janvier. - Assez mauvaise nuit. Constipation. Souffre des jambes. Aucune douleur d'estomac.

Extrait fluide............... XXX gouttes

10 janvier. - Mème état, mais 3 selles. Pas d'extrait.

11 janvier. - Nuit moins calme. Diminution de l'urine.

Extrait fluide................. XL gouttes

13 janvier. - Dans la nuit du 11 au 12 diminution des douleurs, qui ont disparu cette nuit. 3 selles. Urée 10 gr. 248.

15 janvier. - Pas de douleur, malgré la suppression momentanée de l'Actée, mais constipation. Urée 17 gr. 9 par litre.

Extrait fluide............... XXX gouttes

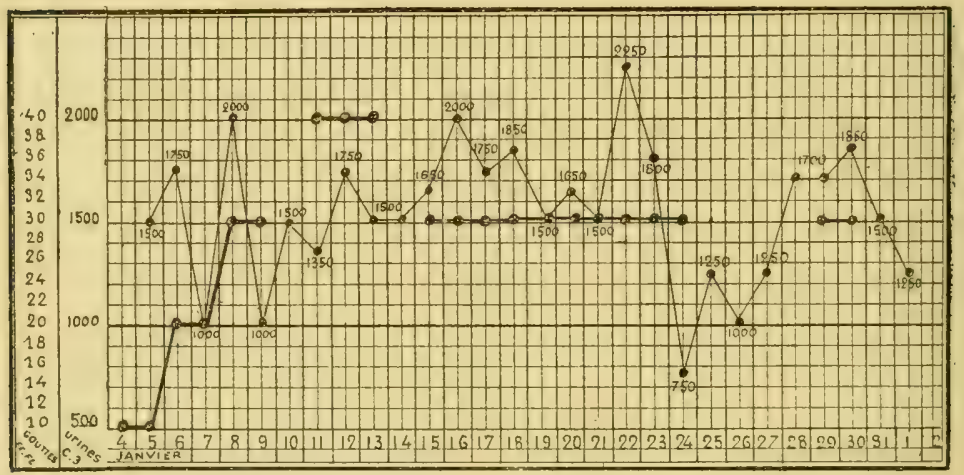

Obs. XX.

17 janvier. - Le 16, crise polyurique. 2 selles. Un peu de souffrance dans les genoux.

18 janvier. -1 selle.

20 janvier. - Diminution des douleurs. Urée 12 gr. 5.

22 janvier. - Le 21, quelques douleurs hépatiques suivies d'un frisson qui dura 3 heures. Douleurs dans les jambes et agitation la nuit.

24 janvier. - Grande amélioration.

2马̈ janvier. - Bonne nuit. Teinte subictérique.

27 janvier. - Coliques hépatiques. Suppression de l'Actée.

29 janvier. - Grande agitation. La malade se plaint de douleurs. dans le jarret et la cheville. 
Extrait fluide................. XXX gouttes

$1^{\text {er }}$ février. - Suppression de l'Actée.

ä février. - Depuis 3 jours amélioration, puis douleurs dans la nuit.

14 fivvier. - Frictions au menthol.

2't férrier. - Grande constipation, huile de ricin.

26 février. - Grande constipation. Douleurs des jambes plus aiguës.

Rem. - L'influence de l'Actée sur la constipation est encore ici bien manifeste. Son influence sédative est inappréciable.

A chaque fois que l'Actée est administrée, la diurèse s'établit; mais son action n'est pas immédiate.

OBS. XXI. - (T'raitement par la poudre d'actéine).

R. B..., âgé de 48 ans, entre à l'hòpital le 2 juin 1896.

HISTOIRE DE LA MALADIE :

$\Lambda$ la suite d'une rougeole, acquise l'hiver précédent, bronchite qui se perpétue jusqu'au 3 juin. Le dimanche 31 mai, douleur dans l'articulation du genou gauche, qui aujourd'hui est le siège d'un épanchement considérable, sans qu'il y ait de douleur spontanée ni provoquée.

ÉtTat actuel :

Poumons. - Gros râles ronflants disséminés dans toute la poitrine Expectoration bronchitique et muco-purulente.

Urines et Cœu normaux.

Fièvre. Éthylisme.

Diagnostic. - Faux rhumatisme ou arthrite tuberculeuse (?).

TraiteMENT :

3 juin. - Deux cachets de 0 gr. 15 centig.

4 juin. - Diminution de la toux et des crachats : même traitement.

5 juin. - Douleurs persistantes. Suppression du traitement. (Le malade atteint de delirium tremens doit ètre isolé.)

Rem. - L'influence sédative de l'actéine est inappréciable.

Ons. XXII. - (Traitement par la poudre d'actéine).

Adolphe C... entre en juin à l'hôpital pour douleurs intercostales vives.

ANTÉCÉd. PERSONN. - Éthylisme.

Diagnostic. - Névralgie intercostale sans cause.

TRAITEMENT :

3 juin. - Deux cachets d'actéine de 0 gr. 15 cent., un le matin, un le soir. 
\& juin. - Le malade souffre moins de ses névralgie, mais douleurs de ventre. Mlême traitement.

5 juin. - Deux cachets. Purgation. Coliques. Cataplasmes laudanisés. suppression des cachets en raison des douleurs stomacales et abdominales. Les douleurs névralgiques sont aussi intenses.

Rem. - Ici encore l'action sédative de l'actéine est inappréciable.

V. au i : OBS. VIII, XVIII. - Arthritisme.

OBS. II. - Ilerpétisme.

Comme emménagogue.

Dès 1818, Davis notait des succès par l'emploi de l'Actæa racemosa, administrée, toutes les 3 heures, sous forme d'un verre à vin de décoction, dans un cas de migraines violentes, dues à un mauvais état de l'utérus.

Gatch Caner semble être le premier auteur tout à fait affirmatif sur les propriétés emménagogues de ce médicament.

" Je ne suis pas surpris, dit-il, que l'attention des médecins ail été appelée sur l'action de cet agent, yui exerce une influence toute spéciale sur l'utérus et ses annexes. Je suis persuadé, depuis longtemps, des propriétés qu'il possède. »

A l'appui de son opinion, Gatcu Carey publie les observations suivantes :

\section{OBS. XXIII. - (CAREY.)}

Miss B..., âgée de 17 ans, est réglée pour la première fois en novem-

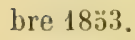

Bientôt un refroidissement supprime la fonction menstruelle, et la région pelvienne devient douloureuse.

Pendant 3 mois l'état s'aggrave : le système nerveux et les fonctions d'assimilation s'altèrent profondément; les symptòmes de dyspepsie et de chlorose surviennent rapidement, et c'est pour les combattre qu'on m'appelle.

Après avoir agi contre la constipation avec de l'huile de ricin j'ordonnai :

T'einture d'Actæa racemosa.

Extrait fluide d'Hyoscyamus.

- - - de valériane.

Bicarbonate de soude.

A prendre une cuillerée à dessert 4 fois par jour. 
Ce traitement, continué avec peu de modifications; pendant six semaines, produisit une diminution notable des symptòmes nerveux, qui avaient presque disparu lorsque les rigles revinrent.

Les fonctions menstruelles se rétablirent régulièrement, quoique quelques périodes fussent retardées et difficiles. Les manifestations nerveuses qui a vaient persisté, plusieurs mois, cédèrent avec l'Actée. La dyspepsie fut combattue et guérie par l'acide chlorhydrique et la gentiane.

\section{OBS. XXIV. - (CAREY).}

Miss D..., âgée de 20 ans, d'apparence scrofuleuse, n'avait pas été réglée depuis 8 mois.

Grande faiblesse et grand abattement, avec douleur dans la région pelvienne et sensation de tiraillement, dans la direction des ligaments ronds. Constipation opiniâtre.

Je combattis la constipation, avec l'extrait fluide de séné, et je prescrivis la dose d'une pleine cuillère à dessert de teinture d'Actée toutes les 6 heures.

Le traitement fut commencé 48 heures avant la période menstruelle attendue, et provoqua l'effet désiré, les règles vinrent et, depuis 3 mois, sont régulières.

\section{OBs. XXV. (CAREY).}

Madame H..., âgée de 20 ans, avait eu de la dysménorrhée pendant 3 ans, et était mariée depuis 2 ans, sans grossesse.

Le 20 juillet, je fus appelé près d'elle, au moment de ses règles; la douleur était déchirante et aussi terrible que dans l'accouchement.

J'ordonnai du camphre et de la morphine toutes les heures, mais le calme ne revint qu'après l'établissement du flux sanguin.

Pendant le mois suivant, elle prit trois fois par jour un drachme de teinture d'Actæa.

Le mois suivant les règles furent faciles. Le deuxième mois, elles furent encore moins douloureuses, et le troisième mois elles ne vinrent pas : la malade était enceinte.

\section{Obs. XXVi. - (Carey).}

Madame G..., âgée de 30 ans, mère de 4 enfunts dont le plus jeune avait 3 ans, avait de la dysménorrhée, depuis son dernier accouchement. Constipation habituelle.

Après l'avoir purgée, je lui ordonnai une cuillère à dessert de teinture d'Actæa et d'extrait fluide séné à prendre 3 fois par jour.

En \& mois, elle était guérie de sa dysménorrhée et a depuis donné naissance à un bébé plein de santé. 
Quelques années après les publications de Gatci-Garer, J. A. Sturson recommande aussi l'Artica racemosa comme un médicament « d'une efficacité toute spéciale dans l'anémie et l'hỵpocondrie puerpérales ».

Il exprime son étonnement d'avoir pu constater la rapidité de son action dans un cas où tout traitement antérieur avait échoué : rapidité dont fut non moins surprise la malade.

Woop croit l'Actée efficace dans les affections chroniques d'origine utérine : dysménorrhée, hystérie.

Telle est également l'opiniun de Georges Rodne (d'Édimbourg), d'après lequel l'Actée est " un médicament ayant une action spéciale sur les organes femelles de la génération, et de la plus grande valeur dans les affections utérines et ovariennes ».

Craig-Balfour s'étonne que le remède soit si rarement prescrit, et publie une note dans laquelle il relate quelques cas où s'affirme l'efficacité de l'Actée.

\section{OBs. XXVII. - (C. Balfour).}

Le premier de ces cas est relatif à une malade guérie par l'Actæa racemosa de douleurs au moment des périodes menstruelles, douleurs et dysménorrhée des plus violentes, et pour lesquelles tout traitement antérieur avait échoué.

\section{OBs. XXVIII. - (C. Balfoun).}

“Un autre se rapporte à une jeune femme, d'une constitution peu robuste et nerveuse, qui souffrait depuis quatre ans d'une dysménorrhée aiguë.

La menstruation, apparue à 12 ans, se continuait difficilement et très douloureusement.

Tous les remèdes ordinaires avaient été employés sans succès, et plusieurs spécialistes avaient déclaré que rien ne pouvait plus agir : la malade devait garder le lit plusieurs jours par mois, et supporter des douleurs qui allaient jusqu'à la syncope.

Quelques années après, ayant par hasard connaissance de cet état, je nroposai qu'un dernier essai fùt tenté. Après un examen attentif, je découvris l'origine nerveuse des symptòmes, et j'essayai l'Actée, dont l'uction est bien connue sur l'utérus et ses annexes.

J'ordonnai $\mathrm{X}$ gouttes de teinture de Cimicifuga, matin et soir, quelques curs avant l'époque présumée des règles, et pendant toute la durée des règles; puis je recommandai à la malade de rester couchée, et de placer 
sur le ventre des cataplasmes sinapisés chauds dès que la douleur commencerait. Dès le commencement du traitement, l'expérience fut couronnée de succès et, en peu de temps, la dose de II gouttes de teinture au commencement de la période menstruelle suflit jour faire disparaitre la douleur, permettre à la malade de rester debout et de ne plus redouter l'approche de chaque période menstruelle. ")

J'ai rencontré, dit Balfour, plusieurs fois des cas analogues, mais j'ai mentionné ceux-ci, vu la durée et la persistance des douleurs.

Brunton a aussi expérinenté l'Actée comme emménagogue, il confirme ce qu'en a dẻjà dit Rodore.

“ L'efficacité, dit-il, avec laquelle l'Actée relève l'état de la malade, et éloigne la douleur de la période menstruelle, le fera prescrire par tous les médecins.

Je l'ai employé dans une série de cas et toujours avec succès, que la dysménor rhée soit d’origine utérine ou ovarienne, l’action est la mème.

Son usage enlève la douleur qui survient aux époques; ses effets toniques surl'utérus et ses dépendances sont non moins marqués.

Dans quelques cas de métrorrhagie, l'Actée remplacera l'ergot avec le plus grand avantage.

Combiné avec le fer, je l'ai souvent heureusement employée dans l'aménorrhée des jeunes filles: le mode d'emploi le plus efficace est de l'administrer à la dose de $\mathrm{XX}$ à $\mathrm{XXX}$ gouttes, 3 fois par jour et 4 jours environ avant la date de la période menstruelle.

Dans les cas d'irritation ovarienne (1), ou selon l'expression de Suxpson, " de mélancolie puerpérale ", il est très simple de l'employer régulièrement.

Lorsqu'il y a une douleur violente des ovaires, une douleur plus ou moins constante dans les reins, de la faiblesse et de la dépression morales quelques doses d'Actée enlèvent la douleur, donnent du ton, relèvent le moral, et font le la malade " une nouvelle femme. »

(1) Irritation ovarienne $=$ hyperhémı ovarienne (LATsoN-TAIT). 
ORS. XXIX. - (BRUnTON).

Ceci m'a encore été absolument démontré dernièrement, par le cas d'une malade rque j'avais vue dans un faubourg de Londres.

Sa mère m'avait dit qu'elle avait eu, 5 ans plus tit, une attaque d'hystérie grave. C'était une jeune femme de 23 ans, jumelle d'une autre fille. Ses parents, gens bien portants, étaient très alarmés à son sujet, craignant pour elle la folie.

Je trouvai la jeune malade pâle et apathique, avec un air malheureux et nonchalant. Elle se plaignait de douleurs de tête, et semblait ne pouvoir supporter la vie, ni ne s'intéresser à rien.

Elle témoignait un peu de douleur et de la sensibilité de l'ovaire gauche et de l'ovaire droit : en somme, c'était une mélancolique ovarienne.

Je lui donnai, trois fois par jour, 30 gouttes de teinture d'Actxa racemosa en même temps que des pilules de fer, car, - bien qu'elle ne fût point anémique, - j'ai souvent observé, dans des cas semblables, le succès du fer associé à l'Actæa racemosa.

En deux jours, elle était beaucoup mieux; à la fin de la semaine c'était une tout autre personne, marchant et chantant à la maison.

Tout anéantissement disparu, la céphalée occipitale disparut ainsi que. les douleurs ovariennes.

\section{OBs. XXX. - (BRUnton).}

Je traitai un cas semblable chez une domestique soignée depuis 6 mois pour une douleur ovarienne, et un malaise plus ou moins continu, la rendant presque incapable de travailler.

J'essayai la teinture d'Actée à la dose habituelle, mais j'ordonnai un vésicatoire sur l'ovaire gauche malade, et j'exigeai qu'un bandage bien. serré fût porté quelques jours.

Le mal qui avait duré six mois s'adoucit, et disparut en une semaine.

Dans un $3^{\mathrm{e}}$ eas j'eus à faire à une dysménorrhée intense.

La malade était une personne de 32 ans, dont l'affection avait été en s'aggravant de plus en plus.

Depuis peu les douleurs rappelaient celles de l'accouchement, la malade se tordant sur son lit en une véritable agonie. Je lui donnai XXX gouttes de teinture d'Actée toutes les 4 heures.

Le jour suivant, qui était le second de la période menstruelle, je la vis et elle était beaucoup mieux. Peuaprès, la douleur avait presque complètement disparu. Elle n'avait pris en tout que 5 ou 6 doses d'Actæa racemosa.

" Je crois en avoir assez dit, pour montrer la grande valeur em ménagogue de l'Actée.

Les conclusions que je voudrais dégager de son emploi sont celles-ci : 
$1^{\circ}$ Elle est certainement un calmant, présentant de gros avantages sur les bromures et les opiacés, dans les douleurs de la dýsménorrhée.

$2^{\circ}$ Dans lat métrorrhagie et la ménorrhagie, e'est un agent régulateur à quelques déceptions près.

3॰Dans la douleur ovarienne et la dépression nerveuse, c'est un spécifique de la plus grande valeur.

La meilleure manière d'administrer la teinture d'Actée est de donner la dose de XXX gouttes, 3 fois par jour, en commençant 4 jours avant la période menstruelle, et continuant pendant toute sa durée. »)

Enfin Millard s'exprime ainsi :

"Chez les jeunes filles, dont le retard des règles et la dysménorrhéc ont pour cause une origine rhumatismale, on se trouve bien de l'extrait fluide d'Actư racemosa, joint à l'iodure de potassium et au sirop de salsepareille.

On prendra de ce mélange une cuillerée à café 3 fois par jour, jusqu'à la ré gularité des règles. »

L’Actée a été recommandée, non seulement dans l'aménorrhée et la dysménorrhée, mais aussi dans la métrorrhagie.

Agirait-elle comme certaines substances, l'ergot par exemple qui, à faibles doses a étẻ vanté pour réveiller la fonction menstruelle, alors qu'à fortes doses il resserre les vaisseaux utérins?

Nous n'avons qu'une expérience personnelle, relative à l'efficacité de l'Actée comme emménagogue. Mais, si cette efficacité est réelle, on comprend fort bien qu'elle donnera de bons résultats dans divers troubles nerveux d'origine utérine, tels que les migraines, la p,leurodynie déterminées par une menstruation troublée.

L'Actée provoquerait-elle des modifications de la circulation locale dans les organes pelviens, comme nous sommes conduits à l'aämettre pour la circulation rénale? Par analogie la chose est possible. Mais nous n'avons pas de données positives à cet égard.

Dans l'une de nos observations (XV), nous avons vu les règles, absentes depuis 3 mois, réapparaître chez une tuberculeuse, sou- 
mise au traitement par l'actéine. Autant qu'on peut conclure d'un cas unique, ce fail serait assez favorable a l'opinion des auteurs ci-dessus.

\section{EMPLOI OBSTÉTRICAL}

Ce n'est que pour rendre complète cette revue des applications thérapeutiques de l'Actée, que nous rapporterons brièvement, l'opinion de quelques auteurs sur son emploi obstétrical.

Selon Koro, l'Actée " diminue ou fail cesser les crampes névralgiques, les douleurs irrégulières de la première période de la grossesse.

Elle calme l'irritabilité réflexe, les nausées, le prurit, l'insomnie, troubles si fréquents de cette période.

Au moment de l'accouchement elle diminue de moitié la période du travail, relìche les fibres utérines et les parties molles du canal que doit traverser le foetus.

Au moment de l'accouchement proprement dit, elle augmente l'énergie et le rythme des douleurs et, de même que l'ergot, assure la contraction utérine après la délivrance. ”

Knorr l'emploie it la dose de $0 \mathrm{gr}$. 7̋̈ centig. d'extrait fluide par jour, pendant les 4 semaines qui précèdent l'accouchement, ou à celle de $\mathrm{V}$ gouttes d'extrait fluide, dans le dernier mois de la grossesse.

Millard croit aussi à l'utilité de l'emploi de l'Actée pendant le travail.

"Nous accordons, dit-il, aux thérapeutes américains que l'Actée a un grand pouvoir sur l'utérus; son action est comparable à celle de l'ergot; elle stimule les contractions de l'utérus chez la femme en travail et active l'expulsion du fœtus.

L'ergot produit une contraction continue, tandis que l'Actée rend plus forte la contraction, sans la prolonger, et par là expose moins la vie de l'enfant.

On dit que l'Actée est utile dans l'expulsion du placenta, en empêchant les accidents du post partum! "

Et il ajoute encore: "J'ai souvent prescrit, quelques semaines 
avant l'accouchement, l'extrait fluide d'Actiea racemosa, à la dose de V à VIII goutles pour favoriser le travail, et les malades ont toujours reconnu la supériorité incontestable de ce médicament. "

La contradiction qui semble bien exister, dans les affirmations de KNonr, au sujet de l'action exercée par l'Actée sur la fibre musculaire de l'utérus, pourrait d'ailleurs faire douter de la véritable valeur de son opinion.

Il est à peine besoin de faire remarquer que les auteurs ci-dessus s'exprimaient ainsi à l'époque où l'ergot de seigle était encore d'un emploi courant en obstétrique; lui chercher des succédanés serait aujourd'hui œuvre bien inutile. 


\section{CHAPITRE VII}

\section{L'ACTÉE COIVIME MODIFICATEUR PHYSIOLOGIQUE}

\section{Esquisse de ses indications thérapeutiques.}

En comparant les résultats fournis par l'expérimentation physiologique (chap. V), et ceux que nous ont donnés les quelques essais cliniques rapportés ci-dessus (chap. VI), il nous est, jusqu'à un certain point, possible d'établir, sur des données sérieuses, l'action de l'Actée sur les diver's systèmes organiques. Certains points restent encore à élucider, mais les grandes lignes de cette action peurent être dès maintenant définies.

\section{Action sur le tube digestif.}

La saveur amère de l'actéine et de l'extrait fluide, l'excitation qu'elle doit déterminer, même à faible dose, sur la muqueuse des premières voies digestives, conduit ì supposer qu'elle doit ètre, dans une certaine mesure, un excitant des sécrétions glandulaires, buccale et stomacale.

Aux doses thérapeutiques nous n'avons, il est vrai, observé chez l'homme aucun fait démonstratif en faveur de cette hypothèse. Quelques analyses de suc gastrique, après repas d'épreuve, ne nous ont pas donné des résultats assez concluants pour être rapportées ici); mais, à doses plus fortes, chez le chien, elle détermine une salivation marquée. La sécrétion du mucus est considérablement augmentice sur toutes les portions de la muqueuse qui ont subi son contact direct, les mucosités ramenées par la sonde œsophagienne, expulsées spontanément ou observées à l'autopsie, sont là pour le démontrer.

Les proprictés thérapeutiques attribuées à l'Actée tendraient, à miori, à la rapprocher des agents nauséeux; même à dose capable 
de provoquer chez l'homme des symptomes ficheux, l'actine ne détermine ni vomissements ni nausées. Dans l'inloxication par l'extrait fluide, on observerait (Brasхани) des nausies, des vomissements, un péristaltisme exagéré, mais pas de fpurgation.

L'actéine, à la dose journalière d'un gramme, loin de déterminer chez le chien de la diarrhie, provorue au contraire une constipation opiniâtre. A dose mortelle, chez le chien et surtout chez les rongeurs, l'actéine détermine une gastro-entérite aiguë.

Il nous est, jusqu'à un certain point, possible, en tenant compte des résultals expérimentaux, de préciser le mode d'action de l'actéine à doses toxiques sur le tube digestif (1).

Son action directe sur l'épithélium n'est pas douteuse: l'épithélium intestinal se détache sous son influence, comme sous celle d'autres toxiques (arsenic, muscarine).

Liexcitation directe des éléments sécriteurs'au moins des cellules muqueuses, provoque leur hypersécrétion, qui est encore accrue du fait de la congestion ultérieure de la muqueuse.

En désquamant les cellules épithéliales, le toxique arrive au contact des fibrilles nerveuses sensitives du splanchnique, dont il détermine l'irritation, d'où une série de réflexes retentissant sur le tube digestil lui-même, sur la respiration ou la circulation. L'injeclion directe d'actéine dans une anse intestinale n'abolit ni ne diminue les mouvements péristaltiques, done elle ne paralyse pas le plexus mỵentérique, ni n'excite les fibres terminales motrices du splanchnique.

L'actéine doit être un agent paralysant des fibres vasomotrices intestinales, ou plutul de leurs terminaisons. La vaso-dilatation paralytique qu'elle détermine provoque une transsudation séreuse, sanguinolente.

Autant que l'on peut conclure des doses toxiques aux doses faibles, il est ì supposer qu'it doses thérapeutiques, chez l'homme, l'acteine doit augmenter les sécrétions et réveiller la contractilité du tube digestif.

(1). Il existe de très nombreux points de ressemblance, en ce qui touche à l'action sur le tube digestif, entre l'intoxication par l'actéine et celle par les Arum, aussi serons-nous très bref, renvoyant pour l'interprétation des faits aux pp. 127-130. 
Les phénomènes d'intolérance gastro-intestinale, observés chez l'homme, semblent bien se rapporter à de la gastrite toxique, soit à de la congestion simple, soit même à des altérations anatomiques phénomènes gastralgiques et entéralgiques très douloureux), et, contrairement à ce qu'on pouvait attendre, la constipation est de règle, tandis que la diarrhée est fort rare. (Il est à remarquer que l'ipéca produit des effets analogues, bien qu'inverses, sur le tube digestif : purgatif à hautes doses, constipant à (loses faibles). A faible dose, l'Actée est un laxatif; à doses un peu fortes, un constipant.

Les phénomènes généraux provoqués par l'ingestion de doses fortes d'acteine sont ceux de l'irritation gastro-intestinale en général. La gastrite aiguë, provoquée par les irritants chimiques, provoque, on le sait, des phénomènes généraux identiques, quelle que soit la nature chimique du toxique irritant.

Si l'on s'en rapporte aux phénomènes d'intolérance observés cliniquement, et aux faits expérimentaux, l'Actée parait surtout agir sur les voies digestives, lorsqu'elle est absorbée par ingestion. A doses thérapeutiques, elle provoque la régularisation des selles chez les sujets atteints de constipation opiniâtre due à l'atonie intestinale; son action sur le tube digestif doit être prise en très sérieuse considération.

On voit par là la valeur qu'il faut attribuer à l'assertion de Sydney Ringer, que l'Actée est sans action sur l'estomac et sur l'intestin.

On pourrail supposer, par analogie avec ce qui se passe pour l'aloine et divers alcaloïdes, qu'une portion plus ou moins notable de l'actéine ingérée est absorbée, passe dans la circulation générale, et n'agit que secondairement sur le tube digestif, par suite des modifications nerveuses et vasculaires qu'elle détermine, ou par suite de son élimination par la muqueuse intestinale ou le foie. Cette hypothèse tombe devant ce fait que l'actéine pulvérulente, insoluble dans l'eau, agit sur les portions de muqueuse avec les- 
quelles elle se troure en contact, avec une rapidité qui ne peut être en rapport avec la durée de son absorption supposée; d'autre part; le contact de l'actéine en solution alcaline avec la surface externe de l'intestin, et sa pénétration dans la circulation générale ne déterminent qu'une certaine congestion du tube digestif, et non la violente gastro-entérite provoquée par le contact direct de la poudre avec la surface interne.

On peut dire, d'une façon générale, que l'actéine à haule dose détermine une irritation violente (une congestion et une lésion plus ou moins considérable selon la sensibilité de l'espèce animale) de toutes les parties du tube digestif, au contact desquelles elle séjourne. Les portions qui, pour une cause quelconque, échappent à ce contact direct, restent indemnes de toute altération. Wême à doses toxiques, l'actéine n'abolit en rien le péristaltisme intestinal.

A dose thérapeutique, l'Actée (ä centig. d'actéine, XXX goultes d'extrait fluide) provoque, d'une manière remarquable la régularisation des selles, mème chez des sujets atteints de constipation opiniâtre: les selles régulières qu'elle provoque ne sont jamais accompagnées de diarrhée, de vomissements, ni de douleurs gastro-intestinales. Elle est bien et longtemps tolérée par les sujets dont le tube digestif et les annexes sont sains; et ce n'est que par un usage prolongé de doses un peu fortes qu'on voit apparaître, chez les sujets sains, des nausées, de la diarrhée et des douleurs gastro-intestinales. Par contre, chez les sujets dont le foie ou l'estomac sont légèrement touchés, un état nauséeux s'établirait facilement sous l'influence de l'Actée, même à faibles doses.

L'action probable de l'Actée sur les sécrétions gastro-intestinales, son pouroir contre l'atonie intestinale semblent des propriétés 'capables de la faire essayer, à titre de stomachique, pour exciter l'appétit et régulariser les fonctions de l'intestin. L. Bruxtor avait déjà, du reste, entrevu cette utilisation possible.

Pour provoquer des phénomènes d'intolérance gastro-intestinale, il semble qu'il faille un usage assez prolongé du médicament; 
il forles doses, ce n'est jamais après sa première administration que surviennent les accidents, ce fait s'exp]ique par la durée du séjour (demontrée par les autopsies) de l'actéine dans le tube digestif.

Nous ne pouvons que très incidemment conclure à une action indirecte exercée sur le foie. Avec de fortes doses cet organe est nettement congestionné; à dose thérapeutique il semble que la proportion de l'urée éliminée dans les 24 heures soit augmentée. Il y a lieu de se demander si ce n'est pas simplement en régularisant les fonctions intestinales, en stimulant par là l’appétit, que l'Actée pourrait avantageusement modifier la nutrition, et en particulier agir sur la production de l'urée.

L'Actée ne semble point agir sur la sécrétion biliaire, ni faciliter l'excrétion des matières de déchet éliminées avec la bile. Une malade (Obs. XX) soumise en traitement par lActée a été prise, au milieu de ce traitement, d'une crise de colique hépatique, longue, douloureuse, avec ictère consécutif.

Les doses capables de déterminer chez l'homme des symptômes fâcheux, ne semblent pas provoquer une intoxication générale. Les phénomènes respiratoires, cardiaques et nerveux observés semblent trouver leur origine dans l'irritation gastro-intestinale.

\section{Action sur l'appareil respiratoire.}

L'Actée est généralement vantée par les auteurs comme un expectorant capable d'augmenter les sécrétions bronchiques (BrAImard, Martin-Fayne, Ciiapman) et semblerait, à ce point de vue, devoir prendre rang près des agents expectorants et en même temps nauséeux. Par contre, DAvis ne l'a jamais vu produire aucune augmentation appréciable des sécrétions.

Dans nos observations cliniques, nous n'avons vu aucune modification nette dans l'expectoration.

Nous ne possédons encore que dec données bien vagues sur le 
mode d'action des substances réputées expectorantes. On peut cependant supposer qu'elles agissent par trois procédés différents.

$1^{\circ}$ Accélération des mouvements des cils vibratiles de l'arbre aérien. Les alcalins (Vincnow), le chlorhydrate d'ammoniaque (Husemans) agiraient de la sorte.

Nous avons essayé de nous rendre compte de l'action exercée par l'Actéine sur les mouvements des cils vibratiles, en opérant chez la grenouille. On sait que des granules pulvérulents, placés dans le pharynx de cet animal, sont peu à peu entrainés dans l'estomac par les mouvements des cils vibratiles de l'œsophage. En plaçant quelques parcelles d'un mélange d'actéine pulvérulente et de poudre de carmin sur le pharynx d'une grenouille, il est facile de s'assurer que les particules colorées sont entraînées aussi rapidement qu’ì l'état normal. D’ailleur's cette action modificatrice sur l’épithélium vibratile ne peut se produire qu'au cas où la substance, entraînée dans la circulation, s'élimine par la muqueuse respiratoire, ce qui semble n'être nullement le cas pour l'Actée.

$2^{\circ}$ Activité plùs grande de la nutrition et régénération facile des épithéliums sains (chlorure de sodium et alcalins).

$3^{\circ}$ Augmentation de l'alcalinité du sang, d'oì anémie légère de la muqueuse respiratoire, neutralisation de l'acidité du mucus stagnant dans les bronches.

Nous ne possédons aucun fait nous permettant d'attribuer à l'Actée l'un ou l'autre de ces deux derniers modes d'action, mais ils seraient bien invraisemblables.

Pour nous rendre compte, par une expérience directe, de l'action de l'Actée sur la sécrétion bronchique, nous avons employé la méthode récemment préconisée par Calvert (1).

Un chien est anesthésié par le chloralose (méthode de RicnET); celte substance ne modifie en rien la circulation. Après avoir placé la tête sur un billot, on ouvre au thermo-cautère la trachée, en lui enlevant un quart de sa circonférence; les tissus enviromnants, une fois bien écartés, on sèche la muqueuse trachéale à l’aide de papier

(1) 1896. CALvert (I.). E/fect of drugs on the secretion from the tracheal mucous membrane (Journ. of. Physiol. vol. XX, nos 2 et 3, pp. 158-164. 21 August). 
burard, et l'on note le temps qu'elle met à redevenir humide; on injecte alors dans la saphène une dose modérée (pour éviter l'action de l'alcool) ( $\breve{5}^{\text {c3) }}$ d'extrait fluide d'Actée ou une dose plus forte $\left(10^{33}\right)$ d'actéine en solution alcaline; puis on essuie à nouveau la muqueuse, et on note le temps qu'elle met à redevenir humide. Ce temps est sensiblement le même qu'avant l'injection. La présence de 30 centigr. de carbonate de soude dans la solution alcaline injectée n'est pas de nature à modifier la sécrétion trachéale, car Rossbaci et Calvert n'ont vu ce sel agir qu'à la dose de $3 \mathrm{gr}$.

Cette expérience nous permet de conclure à l'inefficacité de l'Actée comme modificateur de la sẻcrétion bronchique.

Vantée contre les fluxions pulmonaires et bronchiques, l'Actée pouvait être supposée agir comme anémiant du poumon. Sur les animaux, à doses faibles, comparables aıx doses thérapeutiques, il est impossible de constater à l'autopsie des modifications nettes de la circulation pulmonaire; mais à dose toxique, et très vraisemblablement uniquement par action réflexe, l'Actée détermine, de la façon la plus évidente, une congestion intense du parenchyme pulmonaire.

Il nous a été impossible de constater, dans les essais cliniques chez l'homme, une influence exercée par l'Actée, à doses thérapeutiques, sur les mouvements respiratoires; par contre, son influence, à doses toxiques, sur le rythme respiratoire est des plus évidentes, lorsquielle est administrée par voie digestive.

Les tracés ci-contre, recueillis chez le cobaye, à la période d'état de l'intoxication par administration stomacale d'actéine pulvérisée, méritent d'être analysés avec quelques détails.

(Ils ont été recueillis, à l'aide du cardiographe à double ampoule de Marey (modèle du cobaye). Les lignes de descente correspondent aux expirations forcées).

Les mouvements respiratoires consistent surtout en expirations forcées (I) çà et là (II, III, IV, V, VII), une inspiration forcée, parfois avortée, interrompt l'uniformité de ce rythme si particulier.

Dire qu'il y a expiration forcée active, c'est dire que le centre 


\section{$-319-$}

réflexe bullaire qui préside à ce phénomène n’est pas touché par le poison, que son excitabilité est mise en jeu par le stimulus que lui apporte vraisemblablement le sang asphyxique. Dans le cas qui nous occupe, il n'y a pas à parler d'expiration volonlaire, vu l'état comateux où se trouve l'animal, cette expiration n'est évidemment que réflexe.

Chez un animal où tous les réflexes sont paralysés, nulle expira-
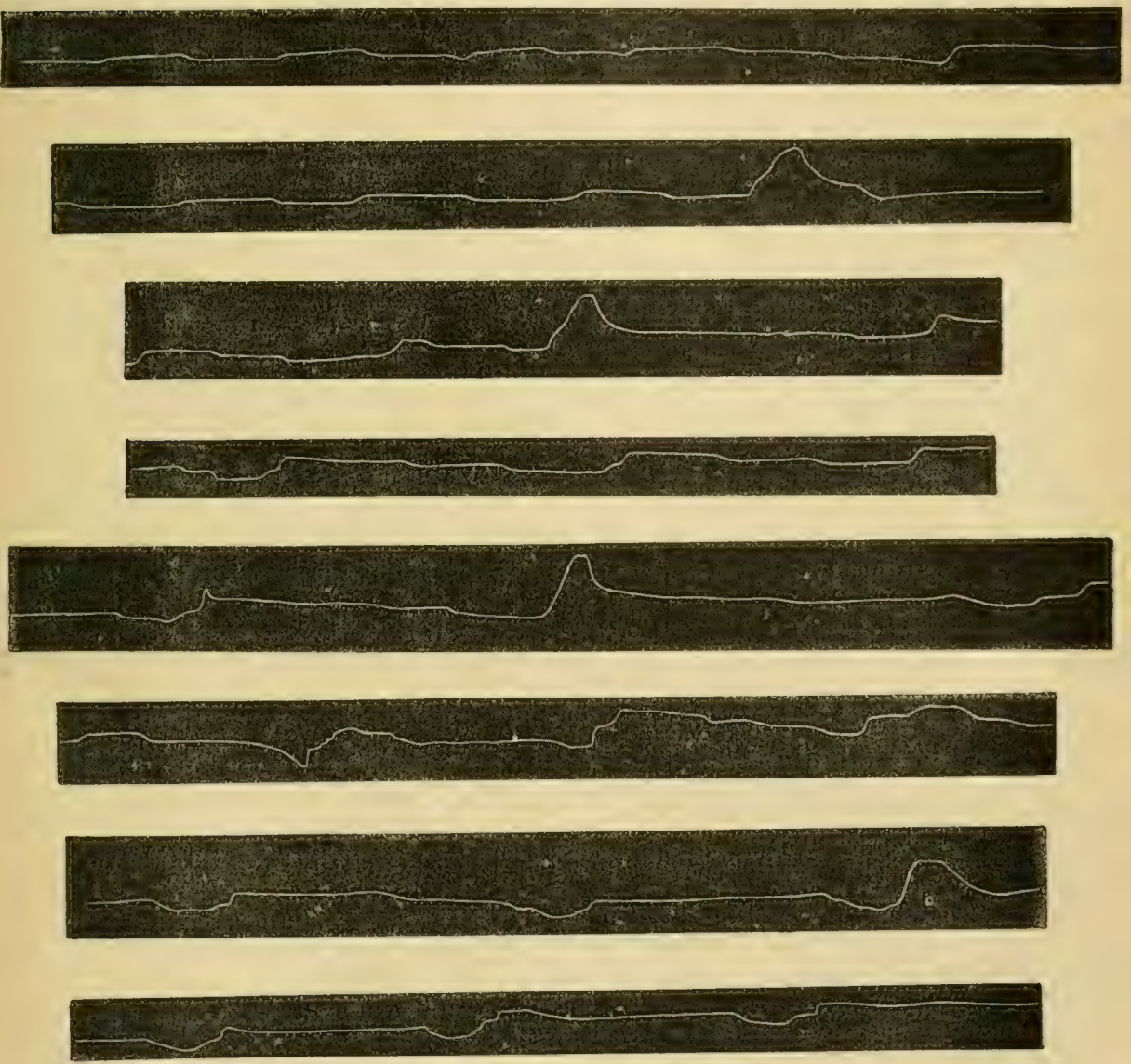

Fig. 75. - Nodifications des mouvements respiratoires chez le cobaye intoxiqué (voie stomacale) par l'actẻine.

(Tracés recueillis avec le cardiographe à double ampoule do Marey, les lignes de descente correspondent à l'expiration,)

(Photog. directe, sans rẻduct.) 
fion ne pes posible que l'expiration mécanique due à l'alasticile des lissus: cest le cas par exemple des animaux profondément chloralisés. Il ne peut, dans ce cas, être question d'expiration forcée.

Done, chez l'animal intoxiqué par l'actéine, les centres réflexes bulbaires ne sont pas atteints.

Il ne semble pas nécessaire d'admettre que le centre respiratoire, du fait de l'intoxication, subit des modifications de son excitabilité, et que, finalement, celte excitabilité se trouve inférieure à la normale. A l'élat normal, le réflexe pulmonaire semble plutôt élre sous la dépendance d'une excitation mécanico-physique des nerfs sensibles du poumon, que d'une excitition chimique du centre respiratoire. Les changements respiratuires observés sont vraisemblablement dus à ce que le centre, normalement excitable, reçoit en rúalité des excitalions différentes. C'est l'explication des modifications respiratoires dues à divers toxiques (chloral, hydrate d’anyle, chloramide, morphine), à laquelle se rattachent A. Loewy (1) et V. Pacuon (2). Dans l'intoxication par ces poisons cérébraux, le cerveau intoxiqué enverrait au centre bulbaire des stimulations moindres qu'à l'état normal, le pouvoir réflexe de la moelle restant intact. Dans notre intoxication, au contraire, à la période ultime, c'est du côté de li moelle que l'apport des stimulations vers le bulbe est diminué.

Administrée par voie digestive, l'Actée ne détermine de modification, du côté de l'appareil respiratoire, que par simple action réflexe. La diminution progressive d'amplitude et de fréquence des mouvements respiratoires, diuns l'intoxication mortelle par injection souscutanée d'extrait fluide chez la grenouille, démontre une action directe de ce toxique sur le centre respiratoire. L'ingestion du même extrait fluide, par voie stomacale chez le cobaye, détermine des modifications des mouvements respiratoires, tres analogues à celles que détermine l'Actéine administrée par la même voie. S'il est incontestable qu'ici encore l'irritalion intestinale retentit sur

(1) A. Locwr. Zur Kenntniss der Erregbarkeil des Athmencentrums (Pflüger's Archiv., XLVL, p. 101).

(2) V. Pachon, Le cerveau et la respiralion (p. 145, Trav. du lab. de Ch. Richet, T. I.) 
l'appareil pulmonaire, nous ne sommes pas en droit d'admettre que, dans la production des phénomènes observés, la part principale ne revienne pas à l'alcool.

L'analyse des modifications de la systole cardiaque chez le lapin intoxiqué par une dose modérée d'extrait fluide, tend bien à faire admettre que des phénomènes de vaso-constrietion se produisent du côté du poumon, phénomènes déterminés évidemment par l'action qu'e xerce le loxique sur les centres vaso-moteur's de l'organe.

L'influence de doses modérées d'Actéine, en solution alcaline, stur la respiration est sensiblement nulle.

Nême à la période d'état de l'intoxication mortelle chez le chien, par injection intra-veineuse d'extrait fluide, le rythme respiratoire n'est sensiblement pas modifié; l'amplitude des mouvements diminue seule, et la respiration continue pendant toute la durée de l'état cumateux, sans qu'il soil possible d'attribuer les légères modifications qu'elle présente, à une autre influence que celle de cel état même.

Nous sommes; sur ce point, en contradiction formelle avec Hutchinson, qui croit à un ralentissement marqué du rythme respiratoire, chez le lapin et le chien (intoxiqués par l'injection intraveineuse d'extrait fluide).

Il est certain que dans l'intoxication par l'extrait, la mort arrive par arrêt de la respiration. Mais il n'y a pas à conclure de l'action de la dose mortelle à celle des doses modérées et thérapeutiques; d'ailleurs il faut faire lla part de ce qui revient à l'alcool dans l'action de l'extrait. Nous pouvons done conclure que l'Actée et l'Actéine n'agissent pas directement sur l'appareil respiratoire, el que les modifications observées au cours de l'intoxication par la voie digestive sont tout entières attribuables à un réflexe dont le point de départ est dans le tube digestif. Ce réflexe doit aussi se produire, bien que très atténué, à la suite de l'administration des doses thérapeutiques.

\section{Action sur la Circulation.}

On a prétendu que l'Actée affaiblit à la fois la force et la fréquence des pulsations cardiaques, qu'elle serail, a ce point de vue, compa- 
rable a l'aconit, qu'elle se comporterail comme un sédatif du syslème artériel.

Daris et Jonsson affirment qu'à dose thérapeutique elle diminue constamment la fores et la fréquence du pouls, et fait tomber la pression artérielle. liardex la regarde plutôt comme un tonique du systeme circulatoire. Pour d'autres, par son action cardiaque et vasculaire, elle se rapprocherait de la digitale.

Dins l'intoxication déterminée chez l'homme par l'extrait fluide d'Actée, Brainard a signalé de la congestion (en particulier de la conjonctive) du côté de la région cépbalique, une augmentation de la pression artérielle, mais aucun ralentissement du pouls, aucune diminution de l'énergie de la systole cardiaque.

Si l'on admettait que l'augmentation de pression artérielle est délerminée par des phénomènes de vaso-constriction plus ou moins généralisée, on s'expliquerait la divergence apparente qui existe entre celle observation et les résultats expérimentaux. Comme l'analyse physiologique nous a conduit à l'admettre, l'Actée apparaitrait comme capable de localiser son action sur certains centres vaso-moteurs.

A doses thérapeutiques, il est impossible d'observer aucune modification cardio-vasculaire chez les malades à système circulatoire sain, soumis à un traitement journalier par l'Actée (extrait fluide, solution d'Actéine). Lorsqu'une duse un peu forte d'Actéine pulvérulente détermine des phénomènes d'intolérance gastro-intestinale, le pouls peut devenir petit, le cœur accéléré, mais ces modifications circulatoires sont entièrement attribuables à un réflexe, dont le point de départ est dans le tube digestif irrité.

Chez une épileptique cardio-aortique (dont l'observation n'a pas íté rapportée au chap. V) nous avons vu survenir des phénomènes syncopaux, à la suite de l'ingestion de 2 cachets de 10 centigrammes d'actéine. Avec un tel médicament, qui agit par voie réflexe sur la circulation, il faut toujours, avant de le prescrire, se préoccuper de l'élat du myocarde; chez les myocarditiques, une dose un peu forle serail de nature ì faire apparaitre des accidents de collapsus. 


\section{La similitude d'action de l'Actée et de la digitale est-elle réelle?}

On a pu croire que l'Actée se rapprochait par son action physiologigue de la digitale (BRowTox). Celle opinion est-ollo justifiec par les expériences sur les animaux ou les essais cliniques sur l'homme? Grâce aux beaux travaux de Potain el Francois Franck, il nous est possible de préciser en quelques mols latetion physiotongirfue de la digilale, à doses thérapentigues. Ello agit sur les pneumogastriques (action cardio-moderatricel de plus, ulle agit direcetement sur le muscle cardiaque et sur les vaisseaux déterminant une action cardiotonique et vasotonique. La diurèse abondante qu'elle détermine n'est pas due à son action directe sur le rein.

Une étude chimique récente, due à Merchies (1), sur la dissocialion, dans ceitains cas pathologiques, de la double action (modératrice cardiaque et tonique vasculaire) de la digitale est la confirmation clinique de ces faits, trop solidement établis pour ne pas prêter à une comparaison fructueuse.

Sur les animaux soumis a l'action de doses faibles ou fortes d'Actée, point de modification durable dans la valeur moyenne de la pression vasculaire. On ne peut surprendre, quel que soit le mode dadministration de la substance, de modification réfle dans le rythme ni dans l'amplitude des contractions cardiaques.

l'Actée déterminerait-elle, à doses thérapeutiques, chez l'homme, une modification vasotonique légère, plus étroitement limitée aux organes abdominaux (comme pourraient le fare supposer ses propriétés réputées emménagogues), en particulier au teritoire rénal (d'où diurèse), la chose est possible, probable même, mais nous ne sommes pas encore à même d'en donner une démonstration précise.

L'analogie avec la digitale ne pourrait être admise que sur ce point spécial, et l'action diurélique, malgre une diflérence d'intensite incontestable, rapprocherait seule les deux plantes, au point de vue thérapeutique.

(1) MrrcinLìn. Les actions dissociées de la digitale, et leur valeur diagnostique et pronostique (Soc. médic. des Hôpit. 1896). 
Faisons observer aussi que la plupart des bronchitiques que nous avons soumis au tratement par l'Actée, avaient un cœur droit auquel il étail indiqué de venir en aide, que, dans la plupart des cas, la digitale aurait été indiquée, et que l'Actée n'a rien fait.

Nous avons vu que les modifications imprimées à la contraction cardiaque, chez la grenouille, par l'extrait fluide sont relativement bien faibles; il n’agit, contrairement a l'opinion d'Hutcursox, ni sur le myocarde, ni sur les ganglions intra-cardiaques. Les faibles modifications qüil imprime à la contraction ne sont guère attriluables qu'il l'irritation locale temporaire qu'il détermine, irritation due en grande partie à l'alcool qu'il renferme.

L'arythmie que détermine l’injection péritonéale du même extrait chez le lapin, a été analysée précédernment; cette analyse nous a conduit à admettre que, très raisemblablement, l'arythmie serait déterminée par des phénomènes périodiques de vaso-constriction pulmonaire. L'action directe de l'extrait sur le cœur lui-mème et sur ses ganglions serait donc nulle.

L'injection intra-vasculaire d'extrait détermine chez le chien une chute brusque, mais momentanée, de la pression artérielle; le rythme du cœur n'est en rien modifié, l'amplitude de sa contraction diminuée. Le principal facteur de la diminution de la pression artérielle est une vaso-dilatation (vraisemblablement de la circulation abdominale). L'Actée agirait donc, dans ce cas, sur les centres vaso-moteurs; Iorsque la dose toxique estatteinte d'emblée, la chute considérable de la pression est évidemment causée en grande partie par l'affaiblissement de la contraction cardiaque. La solution d'Actéine est sensiblement inactive sur le cœur' et les vaisseaux; son injection péritonéile ne détermine chez le lapin que des modificalions insignifiantes et toutes temporaires dans l'amplitude des contractions cardiaques.

Les modificalions circulatoires, observées au cour's de l'intoxication aiguï, consécutives à l'administration de l'Actéine par le tube digestif, ne sont que des phénomènes réflexes. 


\section{Action sur le rein.}

L’Aclée a élé considérée par quelques auteurs comme un diurétique Mantro-lare, par dautres (D.trs) eomme dépourve de toute action à ce point de vue spécial.

Nous n'avons aucune donnée expérimentale pour établir son action sur le rein; l'observation clinique peut cependant nous donner quelques renseignements à cel égard.

Sur beaucoup de malades, l'action diurétique de l'Actéc se manifeste avec une certaine netteté; parfois, on observe de réritables crises polyuriques (les malades qui offrent re phénomène ne présentent ni romissements ni diarrhée; à ce point de vue, l'Actée est assez comparable aux antimoniaux qui, lorsqu'ils ne déterminent ni romissements ni purgation, augmentent prescue toujours la sécrétion urinaire).

L'action diurétique de l'Actée se manifeste surtout quand on l'adminstre pour la première fois, ou lorsqu'on en augmente la dose, elle parait s'épuiser rapidement, et ne se renouvelle que lorsqu'on augmente les doses, ou cqu'on suspend la médication pour la reprendre ensuite.

Nous est-il possible de déterminer par quel procédé l'Actée est diurétique?

Tout diurétique peut ağir sur la sécrétion urinaire, en modifiant soit la pression vasculaire, soit la composition du sang, soit l'innervation glandulaire rénale.

Notre ignorance actuelle sur le roile des nerf's sécrétoires du rein ne nous permet pas d'interpréter, par les modifications de l'innervation glaridulaire rẻnale, l'action d'aucun diurétique.

On sait, depuis les travaux de Ludwig et de son école, que l'augmentation de pression augmente la sécrétion de l'urine, et que la diminution de pression la diminue (la composition du sang demeurant identique).

Tos experiences, arec l'extrat tluide et la solution résineuse, nous ont montré que leur pénétration, à faibles doses, ou mème à doses infiniment supérieures aux doses thérapeutiques, dans la circula- 


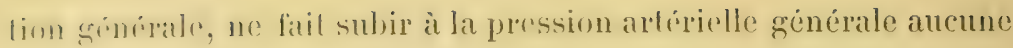
augmentation.

Mais il serait imprudent de conclure à la non-existence de phénominnes vaso-moleurs, d'origine réflexe, dans le ras d'administration de lidir par voir ligestive, phénomenes limités peut-être au rein, el susceptibles de déterminer une élévation de pression dans le silomirule, dont l’intensití ne serail pas suffisante pour retentir sur la circulation générale, mais le serait assez pour déterminer une filtration plus abondante. Cette dernière hypothèse nous parait la plus satisfaisante, au moins pour l'instant.

Une substance dont la présence dans le sang est normale, mais qui est susceptible d'útre éliminés par les reins, peut ìtre diurétique.

Son élimination ne pouvant se faire sans entraîner une certaine quantité d'eau, la polyurie apparaîtra.

L'Actée ne paraît pas agir sur la sécrétion urinaire par action directe sur l'épithélium rénal. Même à dose toxique, chez les animaux, elle ne cause pas trace d'albuminurie. Son action diurétique s'exerce tout aussi bien (et même avec plus d'énergie) lorsqu'elle est administrée sous forme de poudre insoluble, par suite, à peine absorbable, que sous forme soluble et, par suite, capable de pénétrer dans la rireulation gínérale. C'est done surtout en modifiant la circulation rénale qu'elle peut agir sur la sécrétion urinaire. In fail clinifur vient a l'appui de celte opinion. Un de nos malades, en état de crise polyurique, déterisinée par le médicament, a vu cette polyurie s'arrêter du fait d'une asystolie hépatique et rénale; or, si l'Actée agissait sur le rein, indépendamment de la pression vasculaire, par suite de son élimination par l'épithélium rénal la polyurie dans ce cas aurait dû continuer. Le médicament étant impuissant à relever la pression vasculaire, la polyurie s'arrête.

Quoi qu'il en soit du mécanisme de son action diurétiqne, cette action est indéniable. C'est même, avec son action sur le tube digestif, son action thérapeutique la plus nette. 


\section{Action sur les sécrétions.}

Nous n'avons pu nous assurer en aucun cas, dans les expériences physiologiques ou dans les essais rliniques, des propriétés diaphorétiques attribuées à l'Actée (Martyy layme). I) ailleurs, Davis conteste ì l'Actée, d'après son expérience personnelle, toute action sur les sécrétions.

$\mathrm{Si}$, il fortes doses, elle peut déterminer chez l'homme une sudation notable, il est rraisemblable que ce n'est qu'au cas ou elle provoque des phénomènes d'irritation gastro-intestinale; la diaphorèse serait tout indirecte el aussi banale que celle que l'on peut observer au cours de toute irritation des voies digestives.

Nous avons déjà parlé (p. 310̈) de la prétendue action de l'Actíe sur la sécrétion des muqueuses trachéale et bronchique.

\section{Action sur le système nerveux.}

L'Actée est généralement regardée comme un modérateur réflexe: " un des plus parfaits sédatifs que nous possédions, ayant une action profonde sur le système nerveux de la vie organique " Davis. C'est à ce titre, qu'elle serait efficace contre l'épilepsie ct la chorée, pour modérer la toux et comme sédatif de la douleur; à fortes doses, elle déterminerait chez l'homme des phénomènes de dépression nerveuse, de céplualalgie et d'ivresse, des troubles visuels.

Une malade (Obs. XX), traitée depuis quelque temps par l'Aclée a eu, au milieu du traitement, une crise de colique hépatique, tout aussi douloureuse que les précédentes.

Nous avons bien réussi, dans un cas, à atténuer très légèremen des douleurs névralgiques par l'administration de l'Actée; mais, comme anti-épileptique, elle semble se montrer de nulle valeur.

Dans la chorée du chien, où son rôle de modificateur réflexe devrait apparaitre arec netteté, elle s'est montrée totalement ineffcace.

Les expériences sur les animaux ne nous montrent point l'Actée 
ornme loxique à localisation nerveuse; l'extrail fluide détermine, i dose mortelle, une première période, nette surtout sur la Gremonille, plus ou moins marfucie el de courte durée d'excilabilité, puis une seconde priode de dippression. A aucun moment on n'observe de phénomène curarique, le toxique laisse indemnes les terminaisons nerveuses sensitives et les platques motrices, il parait surtout se localiser sur la moelle.

$\Lambda$ la phase terminale de l'intoxication, son action parait se porer sur le bulbe; nous arons déjà parlé de la dépression qu'elle exerce sur les centres vaso-moteurs.

A forte dose, l'extrait fluide provoque, chezle chien et le lapin, une nareose très appréciable; mais il le doit surlout à l'alcool, car l'injection intra-veineuse de l'actéine solubilisée est dépourvue de toute action sur l'axe cérébro-spinal.

L'Actéine administrée par voie digestive se montre dépourvue de toule action sur le système nerveux, abstraction faite des réflexes el de la dépression nerveuse qui succèdent à l’irritation gastro-intestinale qu'elle détermine.

L'apparition de vertige, dans l'intoxication chez l'homme, par l'extrait fluide d'Actée, est commune à bien des intoxications (sulfate de quinine, salicylate de soude, belladone, tabac, alcool).

Ce vertige ne s'observe pas dans l'intoxication rapide, chez les animaux, par voie intraveineuse. Il ne semble done pas qu'il soit déterminé par une excitation toxiquedirecte de lamoelle ou du centre d'innervation du sens de l'espace,

Sa véritable cause doit être cherchée dans l'irritation gastro-intestinale, ce doit être un véritable vertigetoxique, dù à une excitation centripite (probablement par la voie du pneumo-gastrique) de la moelle ou du cervelet. L'exagération de ce vertige, sous l'influence de la station debout, pourrait s'expliquer par une anémie légère de l'encéphale (d'origine vasomotrice). Mais cette explication concorderait mal avec ce fail que la face est fortement congestionnée, alors que ce vertige apparaît, à moins que l'on admette 'fu" lestroubles vaso-moteurs soient inverses dans les vaisseaux de la fince et encéphaliques, ce qui d'ailleurs n'aurait pas lien de 
nous beaucoup surprendre, tant données les modificalions des circulations locales, sous l'influence de l'Actée.

Dans l'intoxication, chez l'homme, par l'extrail fluide die l'Actée, on observe (Branakin) un tremblement et une dureti caractérislique des muscles du dos, des bras et des jambes. Co tremblement est dù a des variations plus ou moins rỵthmiques, de la tonicité musculaire. Il est à rapprocher du tremblement toxique dù à la nicotine, qui, selon Vulpian, serait d'origine bulbaire.

Les excitations successives parties de la moelle, et qui déterminent le tonus musculaire, ne sont plus, au cours de l'intoxication, d'égale intensité; la trémulation est due, rraisemblablement, à la prédominance temporaire, àintervalles réguliers, des muscles antagonistes.

Ce trouble médullo-bulbaire est encore attribuable it un réflexe d'origine gastro-intestinale.

La céphalalgie, le priapisme, l'agitation, signalés par le même auteur, sont justiciables de la même explication.

A doses modérées chez les animaux, à doses thérapeutiques chez l'homme, l'Actée n'apparait nullement comme un modificateur rénexe et un sédatif du système nerveux.

\section{Conclusions.}

En résumé, l'efficacité thérapeutique de l'Actée dans les nombreuses affections contre lesquelles elle a été rantée, reste encore à vérifier; il ne semble pas, contrairement à l'opinion généralement admise en Amérique, que ce soil, à doses thérapeutiques, un modérateur reflexe, un modificateur cardio-vasulaire ou respiratoire, digne d'etre substitué aux agents courants de notre thérapeutique européenne. C'est à titre de régulateur des fonctions gastro-inteslinales et de diurétique, que l'Actée et son principe actif l'actéine méritent dattirer l'attention. Les 29 observations personnelles présentées dans ce travail n'ont nullement la prétention d'établir les indications précises et l'efficacité incontestable de l'Actée, elles sont beaucoup plutit de nature à mettre en doute, ou à contredire les assertions jusqu'ici émises par les auteurs américains. C'est à des 
essais plus nombreun, plus systématiquement localisés à des points spéciaux, et que nous nous promeltons de poursuive, de nous fixer délinitivement sur la valeur thérapeutique de l'Actée. L'isolement de son principe actif, nettement défini maintenant au point de vue chimique, les expériences physiologiques, soit avec l'extrait tutal, soit avec l'actéine et quelques observations cliniques nous ont paru être des documents assez sérieux pour constituer la deuxième partie de ce travail.

BibL. 1823. - GARden (T. S.). Onthe use of the Actæa racemosa in phtists pulmonalis. (Am. M. Recorder, Phila., VI, pp. 609-13.)

1827. - MEARs (G. W.). An inaugural essay on the properties and effects of the Cimicifuga racemosa (black snake root) (submitted to the faculty of Jefferson medic. College. Phila.; Month. J. M. and S. I, 153-169, 1 pl.).

1833. - Tully (W.). Actæa racemosa (Boston M. and S. J., VIII, $133 ; 149)$.

1845. - Wooten (H. V.). Cimicifuga in nervous diseases (South. II. and S. J., n. s. I, 569-572).

1848. - Johnson (F. N.). (Reports N. Y. Hospital.)

Davis (N. S.). Report of the Committee ou indigenons medical Bolany. Cimicifuga racemosa. (Trans. Am. med. Assoc., vol. I, p. 351.)

1851. - GARDEN (T. J.). Observations on the remedial powers of the Cimicifuga racemosa in the treatment of incipient plitisis and chorea (Stethoscope and Virg. M. Gaz., Richmond, 1851, I, 317-322).

1855. - Gatch Carey (H.). Black Snakeroot : Its emmenagogue properties (The Medical Counsellor, vol. I, $\mathrm{n}^{\circ}$ 1, $6 \mathrm{Ja}$ nuary, p. 1-3. Columbus).

1856-7. - Adolphus (J. F.). Cimicifuga racemosa (Penins. J. M. Ann Arbor, Mich., IV, 12-15).

1858. - O'Connon (J. D.). Corbis segminis. (Cincin. Lancet and Obs., I. 200-205).

1860. - Smpson (J. V.). Clinical Lectures on the Diseases of Women (Medical Times, vol. II).

1861. - Simpson. Gimicifuga racemosa (Chicago M. Exam., II, p. 331-38). 
1861. - Parnish (E.). Therapeutic. and pharmaceutic. notes on Cimicifuga (Med. and Surg. Reporter, Phila., V, p. 531).

1862. - PerGY (S. R.). Cimicifuga (Bull. N. York Acad. M. I, p. 188).

MAG-Donald. Actæa racemosa (British Medical Journal, London, vol. I, p. 6ö. - Edinburgh medical Journal. - Dublin Medical Press).

1867. - Monse (D.-A.). The action and therapeutical value of Cimicifuga racemosa (Med. and Surg. Reporter, Phila., XVI, pp. 104-6).

1868. - Adolphus (J.). Cimicifuga racemosa (Med. and Surg., Reporter, Phila, XVIII, pp. 337-41).

1872. - Nornis (G.-D.). Remarks in Alabama State Medical Association on the use of infusion of Cimicifuga racemosa as a preventive of small pox (Atlanta M. and S. J., X, p. 17).

1873. - Bailey (F.-H.) Actæa racemosa (Med. and Surg. Reporter, Philadelphie, XXVIII, p. 109).

1876. - Loving (S.). Remarks on the therapeutic application of Cimicifuga racemosa (W. Virg. M. Student, Wheeling, I, p. 77-8\%).

1876. - Pearson (N.-T.). On Cimicifuga indirect medication (Chicago MI. Times, VII, p. 479-81).

1880. - Sydney Ringer. A Handbook of Therapeutics (eight edit. London (Lewis), pp. 432-435).

1885. - KNonr (J.-S.). The influence of Cimicifuga racemosa on parturition (Chicago, M. J. and Exam., 1, p. 287-91. - (J. Am. M. Ass., Chicago, IV, p.405̈).

1886. - PATTON (A.-S.). Actæa racemosa for Tinnitus. (Medical Press and Circular, t. 42, p. 136).

1888. - Colvin (D.). On the use of Cimicifuga in chorea, rhumatism, and pregnancy (Med, and Surg. Reporter, Phila. IV, p. 769.)

1889. - Unaig Balfour (Lancet, vol. I).

LAUder-Brunton (T.). Traite de pharmacologie, de therapeutique... (trad. frane., p. 923).

1892. - Brunton (J.). The use of Actæa racemosa in dysmenorrhæa and ovarian irritation (Practitioner, London, XLVIII, p. 263̈-268).

1896. - Millard (F.-R.). Somes uses of Cimicifuga racemosa 
$-332-$

(Med and Surg. Reporter, 18, January, LXXIV, $n^{\circ} 3$, p. 70-72).

HildReth (Сн.). (New-York Journal of medecine and collateral Science, vol. V, p. 314).

1896. - Hewelke (A.). (Kron. le R., n 18).

1897. - Id. (A.). (Ther. Wchnschrft, $\left.n^{\circ} 6, \mathrm{p} .134\right)$. 


\section{ADDENDA}

P. 245.

Bibl. ICON, 1761-1845. - OEder. Flor. Danic. (14 vol, fol. 3 tab. color. IIavnia. $3,505$.

1780. - Muelter (J.-S.) Icones plantarum (gr. fol., tab. color., t. LII).

1790-1814. - Shith and Sownerby. English Butany (gr. $8^{\circ}$, tab. color., 19,1298)

1798-1849. - Stura (J.) Deutschlands Flora. (8º, tab. color. Leipzig. $11 \times 44$.

1803-46. - IAYNE.(F.-J.). Getreue Darstellung und Beschreibung der Arzneigewächse. (14 vol. 4º, Berlin, tab. color., 13,32.)

1811-18. - Schranck (F.). Flora monacensis (gr. fol. tab. color., 21,23).

1816-1830. - Dict. des sc. nat. (LX vol. $8^{\circ}$, Paris, Strasbourg, Levrault, 3.)

1817-1818. - CunTis. Flora Londinensis. (New edit. by Graves and W. J. Hooker, London, tab. color. 2,35.)

1828. - Nees von Esebeck, Weihe, iVolter und Funke. Plantæ medicinales (fol. Dusseldorf, tab. color, , 7).

1832. - Woodville and Hooker, Medic. Bot. (London, tab. color. 4,249.)

1834-70. - Reichenrach (H. et G.). Icones Foræ German. (Cent. VII p. 8, p. q. t. XII.)

1834-1843. - Baxten. Britisch phanerogamous Botany (g. $8^{\circ}$ tab. color. London, 4,261).

1838. - Brandt und Ratzeburg. Deutschland's phanerogamisch Giftgewächse. (4º Berlin, tab. color. 7,20.)

1843-33. - Nees von Esenbegh et Caspary. Genera plantarum Flor. German. $\left(8^{\circ}\right.$, Bonnæ, tab. nigr. $3 \times$.)

1844-64. - PlÉE (Fr.) Types de chaque fam. (livr. XXVII).

1846. - Dughesne (C.-A.). Répert. des pl. utiles. (Atlas, pl. V.)

Bot. Mag., t. 2432.

Baillon (H.), Dupuis (A.) et RÉvoil (O.). Flore Médicale du $X I X^{\mathrm{e}}$ siècle (t. I).

1876. - Baillon (H.). Dict. de Bot. 


\section{EMENDENDA}

P. 40. La fig. 28 a été placée par erreur la tête en bas.

P. 113. La paralysie déterminée par l'alcaloïde d'Arum est due à la localisation du toxique sur les plaques motrices; il s'agit d'un toxique à action curariforme comme la conicine type.

P. 153. Dans les jeunes pousses d'Oseille la proportion d'acide oxalique doit être plus forte, car il résulte des recherches de Berthelot et André, que nombre de plantes, au début de la végétation, renferment des quantités relativement considérables de cet acide.

P. 176. nostras et non nostra.

P. 198. Fig. 62. Par erreur, lors de la gravure, le diamètre tangentiel de la zone libérienne du faisceau a été trop réduit. Cette réduction, par rapport aux diamètres de l'arc péricyclique et de la zone ligneuse, bien que marquée sur certains faisceaux, n'est jamais aussi accusée.

P. 2a1. Fig. 71, le tracé II a été placé par erreur la tête en bas.

P. 2H4. Fig. 72, le tracé VII a été placé par erreur la tête en bas.

P. 259. la (à supprimer).

P. 263. Fig. 74, le tracé III a été placé par erreur la tête en bas. 


\section{TABLE}

\section{PREMIERE PARTIE}

\section{Recherches médicales sur le genre Arum.}

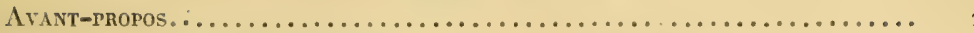

Chap. I. - Étude botanique :

A. Arum maculatum L. et italicum Mrs........... 5

B. Arisarum vulgare TARg. Tozz................ 49

Chap. II. - Étune chmove :

A. Arum maculatum et italicum............... 61

B. Arisarum vulgare...................... 81

Crar. III, - Étude expérimentale des propriétés toxiques des Arum... 8 \&́

Cirıp. IV. - Étude expérimentale des propriétés toxiques de l'Arisarum vulgare................................ 115

Cirap. V. - Mode d'action et nature des principes toxiques des Arum... 121

CHap. VI. - L'intoxication spontanée par les Arum............... 138

Chap. VII. - L'intoxication par l'oseille :

Comparaison avec l'intoxication par les Arum......... 149

Char. VIII. - Les Arum au point de vue médico-légal.............. 164

\section{DEUXIÈME PARTIE}

\section{Recherches médicales sur le genre Actæa.}

Chap. 1. - Ëtude motanique :
A. Actæa spicata $\mathrm{L}$

B. Actæa racemosa $\mathrm{L}$

Chap. II. - Étude chimrue :
A. Actæa spicata..

B. Actæa racemosa............................ 211

Cнат. III. - Pharmacologie. Posologie..................... 226

Crisp. IV. - L'Actæa spicata au point de vue médico-légal.......... 234

Chap. V. - Étude phrsiologi@Ue :

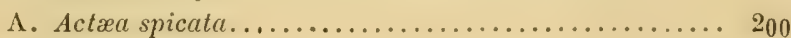

B. Actæa racemosa.......................... 200

Chap. VI. - L'Actæa racemosa comme agent thérapeutique.......... 268

Chap. VII. - L'Actée comme modificateur physiologique. Esquisse de ses indications thérapeutiques..................... 312

Paris. - Imprimerie F. Levé, rue Cassette, 17. 









QL 38 . A7 C C45 45 Botanical Garden Library
Chauliaguet. Juliet/Etudes medicales cur

gen

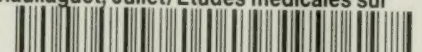

|||||||||||||||||||||||||||||||||||||||||||||||||||||

35185000724904 
A

S1:

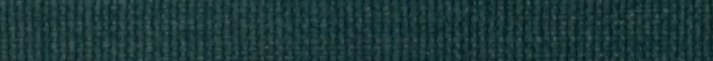

[5.

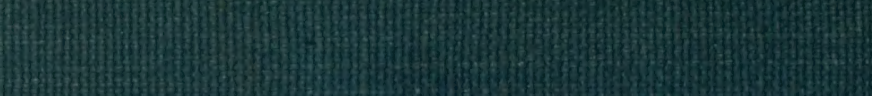

9.

S4) 